\title{
Molecular mechanisms underlying cortical (mal)formation: case studies of ESCO2 and EXOSC10
}

\author{
Dissertation \\ for the award of the degree \\ "Doctor rerum naturalium" \\ of the Georg-August-Universität Göttingen
}

within the doctoral program Cellular and Molecular Physiology of the Brain of the Georg-August University School of Science (GAUSS)

Submitted by

Pauline Antonie Ulmke

Born in Aachen, Germany

Göttingen, 2021 


\section{Thesis Committee}

Prof. Dr. Jochen Staiger

Department of Neuroanatomy, University Medical Center Göttingen

Dr. Sebastian Kügler

Department of Neurology, University Medical Center Göttingen

Prof. Dr. Andre Fischer

Department for Psychiatry and Psychotherapy, University Medical Center Göttingen

\section{Members of the Examination Board}

Prof. Dr. Jochen Staiger

Department of Neuroanatomy, University Medical Center Göttingen

Dr. Sebastian Kügler

Department of Neurology, University Medical Center Göttingen

\section{Further members of the Examination Board}

Prof. Dr. Andre Fischer

Department for Psychiatry and Psychotherapy, University Medical Center Göttingen

Prof. Dr. Ralf Heinrich

Department for Cellular Neurobiology, University of Göttingen

Prof. Dr. Tiago Fleming Outeiro

Experimental Neurodegeneration, University Medical Center Göttingen

Prof. Dr. Bernd Wollnik

Institute of Human Genetics, University Medical Center Göttingen

Date of oral examination: $23^{\text {rd }}$ of August 2021 


\section{Declaration}

I hereby declare that the $\mathrm{PhD}$ thesis entitled "Molecular mechanisms underlying cortical (mal)formation: case studies of ESCO2 and EXOSC10" was written independently, with no other sources and aids than cited.

Pauline Antonie Ulmke

Göttingen, $7^{\text {th }}$ of July 2021 


\section{Table of Contents}

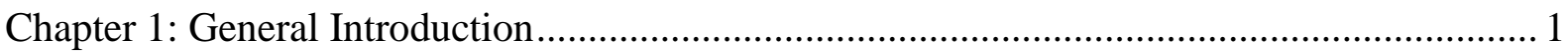

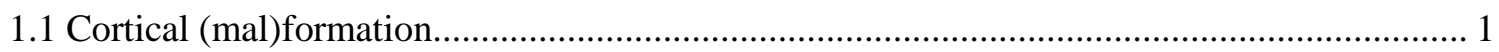

1.2 ESCO2 is essential for proper sister chromatid cohesion in cell division................................. 4

1.3 Role of ESCO2 in human malformation syndromes ........................................................... 5

1.4 The exosome complex regulates gene expression post-transcriptionally ................................ 7

1.5 Role of the exosome complex in neurodevelopmental disorders ........................................... 11

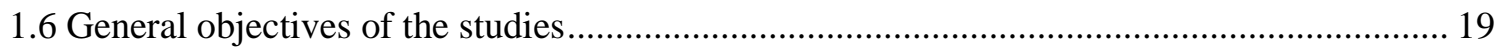

Chapter 2: Molecular profiling reveals involvement of ESCO2 in intermediate progenitor cell

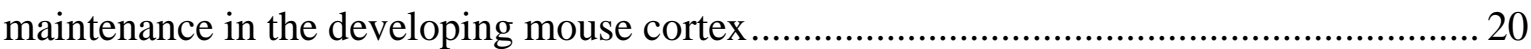

Chapter 3: Post-transcriptional regulation by the exosome complex is required for cell survival and forebrain development via repression of P53 signaling ......................................... 50

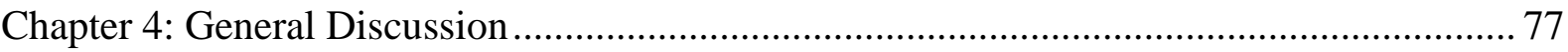

4.1 ESCO2 and other IPC-related factors in cortical (mal)formation .......................................... 77

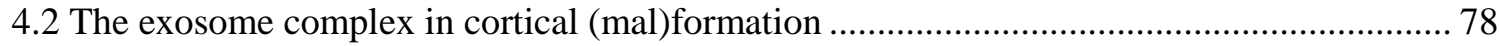

4.3 Future directions: EXOSC10 in SHH and WNT/ $\beta$-catenin signaling .................................... 79

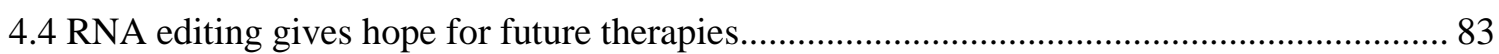

4.5 EXOSC10 might be beneficial for a novel RNA editing tool ............................................. 85

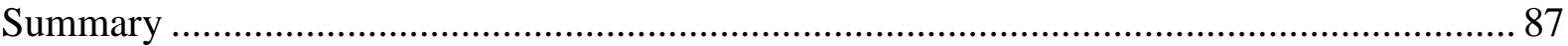

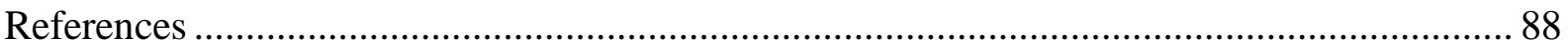

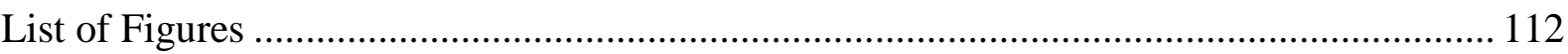

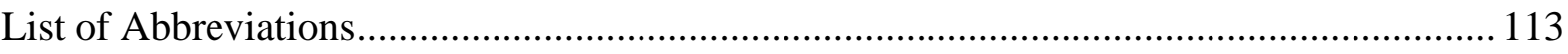

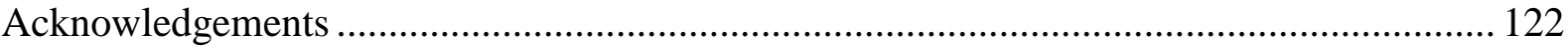

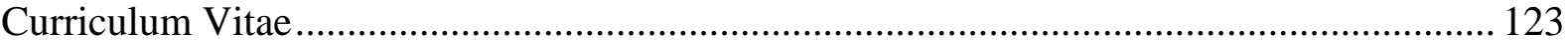




\section{Chapter 1: General Introduction}

\subsection{Cortical (mal)formation}

\subsubsection{Development of the cortex}

In the developing vertebrate central nervous system, neural stem cells (NSCs) and progenitor cells generate neurons in the process of neurogenesis, which is tightly regulated in a temporal and spatial fashion along the different body axes (Gotz and Huttner, 2005; Taverna et al., 2014). Neurogenesis is followed by migration of neurons. The formation of dendrites, axons and synapses is the basis for the generation of neuronal connections and networks (Komuro and Rakic, 1998; Kriegstein and Noctor, 2004).

The earliest progenitors of the cortex are called neuroepithelial cells (NECs). NECs show typical epithelial features and form the neuroepithelium, which looks layered ('pseudostratified') due to the apico-basal movement of NEC nuclei during the cell-cycle (Takahashi et al., 1995; Taverna and Huttner, 2010). NECs expand their pool by symmetric proliferative divisions (Taverna et al., 2014).

At the beginning of neurogenesis, NECs divide asymmetrically to generate radial glial cells (RGCs) with neuroepithelial and astroglial characteristics (Gotz and Huttner, 2005; Kosodo et al., 2004; Malatesta et al., 2000; Miyata et al., 2004). With the generation of neurons, the neuroepithelium transforms into a tissue with several layers of cells. RGCs are found in the ventricular zone (VZ) of the cortex, the most apical cell layer that lines the ventricle (Noctor et al., 2002; Taverna et al., 2014). In rodents, the time window of neurogenesis in the developing cerebral cortex extends from embryonic day 10.5 (E10.5) to mostly E16.5, followed by gliogenesis (Gotz and Huttner, 2005). First, RGCs divide asymmetrically to self-renew and produce a projecting neuron in direct neurogenesis (Fig.1.1A) (Gotz and Huttner, 2005; Huttner and Brand, 1997). The first neurons (born around E10.5 in the developing mouse cortex) form the preplate (Molyneaux et al., 2007). The preplate then splits into the marginal zone (MZ) and the subplate (Hevner et al., 2001; Magdaleno et al., 2002; Molyneaux et al., 2007; Wood et al., 1992). The cortical plate (CP) forms in between the preplate and the $\mathrm{MZ}$ and develops into the multilayered neocortex later (Agirman et al., 2017; Molyneaux et al., 2007). The first waves of neurons populate mainly the lower cortical layers (LLs) L6 and L5 (Molyneaux et al., 2007).

As corticogenesis proceeds, indirect neurogenesis becomes predominant as RGCs produce intermediate progenitor cells (IPCs) that delaminate from the VZ and invade the subventricular zone (SVZ) (Fig.1.1A) (Haubensak et al., 2004; Noctor et al., 2001; Noctor et al., 2004). In overlapping waves, IPCs undergo limited cycles of symmetric divisions to generate the majority of cortical neurons, which populate the upper layers (ULs) L4-L2 (Haubensak et al., 2004; Kowalczyk et al., 2009; Letinic et al., 2002; Noctor et al., 2004; Tarabykin et al., 2001). The MZ develops into L1, which is populated by tangentially migrating Cajal-Retzius cells from outside of the neocortex, mainly from the cortical hem (Marin-Padilla, 1978; Molliver et al., 1973; Yoshida et al., 2006). 


\subsubsection{Developmental defects leading to cortical malformation}

Any defect affecting cortical development at any stage can result in malformation of cortical development (MCD) (Raybaud and Widjaja, 2011; Subramanian et al., 2019). MCDs are a heterogeneous group of neurodevelopmental disorders. Different defects were named according to their morphologic feature, e.g. microcephaly for a small brain, macrocephaly for a big brain, lissencephaly for a smooth brain or polymicrogyria for a brain with too many small gyri (Raybaud and Widjaja, 2011). Combinations, e.g. microlissencephaly (brain with reduced size, thick cortex and defective gyration), occur as well. Microcephaly occurs non-syndromic or syndromic when it is part of more complex syndromes, for instance it can be associated with brainstem and cerebellar malformations as in pontocerebellar hypoplasia.

Microcephaly can be evident at birth (primary microcephaly) or occur postnatally (secondary or postmigrational microcephaly), usually within the first years of life (Juric-Sekhar and Hevner, 2019; Passemard et al., 2013). Whereas primary microcephalies reflect an imbalance between progenitor cell production and cell death, secondary microcephaly often implies neurodegeneration or other cell death (Passemard et al., 2013).

Based on the analysis of mouse models, different mechanisms were identified to be involved in the development of microcephaly (Fig. 1.1) (reviewed by Bizzotto and Francis, 2015). These include defects in RGs proliferation (Fig.1.1B), premature differentiation of RGs into post-mitotic neurons at the expense of IPC genesis (Fig.1.1C), increased cell death (apoptosis) (Fig.1.1D) and defects in neuronal differentiation (Fig.1.1E). In all of these defects, the affected cells generate less progeny cells, finally, leading to a decreased number of neurons.

The molecular mechanisms underlying these defects causing microcephaly are diverse. Environmental factors can lead to primary microcephaly, like intrauterine infections (e.g. Zika virus, rubella or toxoplasmosis), drugs (alcohol, cocaine or antiepileptic drugs) or other factors during pregnancy (e.g. malnutrition or placental insufficiency) (Devakumar et al., 2018; Oliveira Melo et al., 2016; Passemard et al., 2013). Additionally, genetic mutations can cause microcephaly and other MCDs (Barkovich et al., 2012; Juric-Sekhar and Hevner, 2019; Passemard et al., 2013). Several gene mutations associated with primary microcephaly are involved in pathways affecting the cell cycle phases of mitosis and thereby change the rate of neurogenesis, e.g. mutations in genes involved in cell cycle progression and checkpoint control, centrosome maturation and duplication, mitotic spindle formation and DNA repair (MCPH1, CDK5RAP2, CENPJ, NDE1 and WDR62) (Alkuraya et al., 2011; Bakircioglu et al., 2011; Bilguvar et al., 2010; Feng and Walsh, 2004; Thornton and Woods, 2009; Yu et al., 2010). Besides, also gene mutations affecting the progenitor proliferative capacity can lead to a reduced number of neurons and primary microcephaly (ASPM and STIL) (Desir et al., 2008; Kousar et al., 2010; Kumar et al., 2009; Papari et al., 2013).

Defects in neuronal migration are often linked to lissencephaly, including mutations in PAFAH1B1 (formerly LS1), DCX, ARX and REELIN (Cardoso et al., 2002; Hong et al., 2000; Kitamura et al., 2002; Pilz et al., 1998; Pramparo et al., 2010). However, also microcephaly can be associated with abnormal migration as several factors that control proliferation of neural progenitors are essential regulators of neuronal migration (Passemard et al., 2013). For instance, the intermediate progenitor specific transcription factor TBR2 (EOMES) is thought to be involved in neuronal division and migration and its silencing leads to microcephaly (Baala et 
al., 2007). Besides, genes involved in microtubule formation and microtubule-associated proteins, which are critical for cell migration, have been linked to microcephaly and various other MCDs (TUBA1A, TUBB2B, TUBB3, TUBG1, DYNC1H and KIF5C) (Abdollahi et al., 2009; Bahi-Buisson et al., 2014; Di Donato et al., 2017; Kumar et al., 2010; Poirier et al., 2007; Poirier et al., 2010).

A study, which investigated the genetics of microcephaly in 62 patients, identified transcriptional regulation and DNA damage response pathways as the most frequent affected pathways in both, primary and secondary, microcephaly (Boonsawat et al., 2019). Centrosomeassociated pathways were only found in primary microcephaly, corresponding to their function in cell division during neurogenesis. Whereas the inheritance of primary microcephaly was mainly recessive suggesting a complete protein absence as the most frequent cause of primary microcephaly, dominant de novo mutations were often observed in secondary microcephaly indicating that haploinsufficiency may be common for secondary microcephaly (Boonsawat et al., 2019).

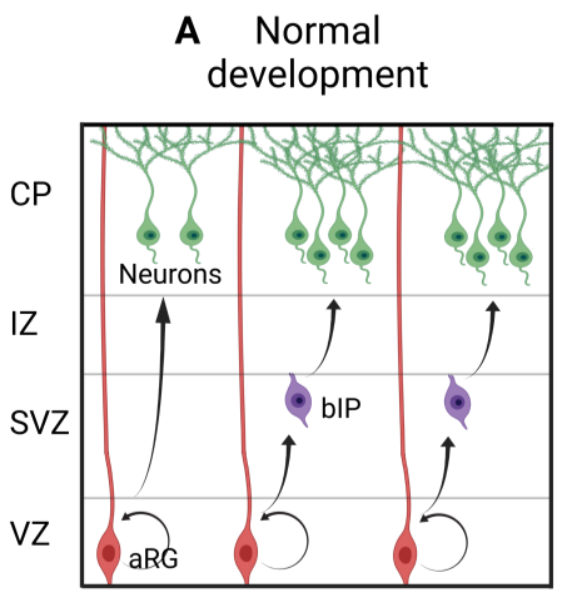

Microcephaly with defects in:

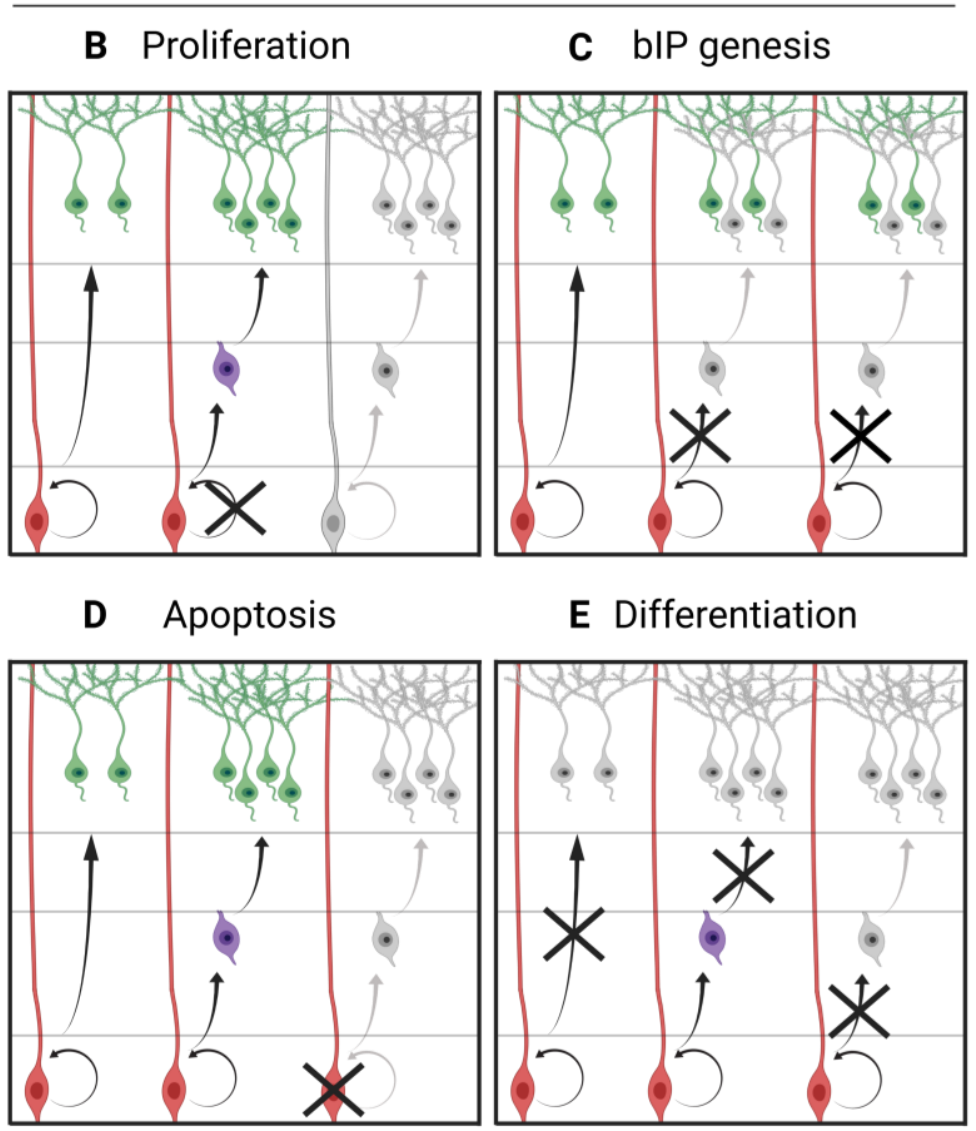

Figure 1.1 Mechanisms leading to primary microcephaly in mouse models. (A) In normal cortical development, apical radial glial progenitors (aRGs) self-renew in the ventricular zone and may generate neurons directly. Additionally, aRGs also produce basal intermediate progenitors (bIPs), which divide in the subventricular zone (SVZ) to generate more neurons. Due to their relative rarity in the mouse cortex, the basal radial glial progenitors (bRGs) are not included in this scheme. (B-E) Potential mechanisms leading to microcephaly, based on analyses of mouse models. They include defects (indicated by X marks) in the proliferation of aRGs (B), premature differentiation of aRGs into neurons at the expense of bIP genesis (C), increased cell death (D), and impairments in neuronal differentiation (E). The affected cells do not generate progeny cells (marked in light gray). Abbreviations: VZ, ventricular zone; SVZ, subventricular zone; CP, cortical plate; aRGs, apical radial glial 
progenitors; bIPs, basal intermediate progenitors. Created with BioRender.com. [Adapted from (Bizzotto and Francis, 2015)].

\subsection{ESCO2 is essential for proper sister chromatid cohesion in cell division}

\subsubsection{Cell division}

The aim of cell division is to create two genetically identical daughter cells from one parent cell (Mitchison and Salmon, 2001). The cell grows and duplicates its genetic information during the interphase of the cell cycle. Afterwards, the condensed chromosomes comprise two identical sister chromatids each and mitosis, the step of the cell cycle in which the cell divides into two daughter cells, can start (Scholey et al., 2003).

The mitotic spindle is the cytoskeletal structure including microtubules and multiple mitotic motors responsible for the distribution of identical copies of the replicated genome to the daughter cells (Mitchison and Salmon, 2001; Scholey et al., 2003; Sharp et al., 2000; Wittmann et al., 2001).

The first phase of mitosis is the prophase in which the duplicated centromeres of the chromosomes migrate around the nucleus. Subsequently, the nuclear envelope breaks open enabling the microtubules to align the chromosomes to the cell equator in prometaphase (Kapoor and Compton, 2002).

By metaphase, the pairs of sister chromatids are placed on the spindle equator towards opposite spindle poles (Kapoor and Compton, 2002). Microtubules are arranged in four sets linking the spindle poles to the cell cortex (astral microtubules), the chromosome arms to the poles (chromosomal microtubules), the poles to the kinetochores (kinetochore microtubules) and the two poles to each other (interpolar microtubules) (McIntosh et al., 2002; Scholey et al., 2003; Wittmann et al., 2001).

Next, the cohesion between sister chromatids is abrogated, so that they can be moved to opposite spindle poles in anaphase (Shah and Cleveland, 2000). The spindle poles move further apart as well in a process named pole-pole spacing (Brust-Mascher and Scholey, 2002; Sharp et al., 2000). At the same time, the spindle sends a signal to the cell cortex that determines the positioning of the contractile ring (Glotzer, 2001; Robinson and Spudich, 2000).

In telophase, the nuclear envelope reassembles around the sets of decondensing, segregated sister chromatids (Guttinger et al., 2009). The contractile ring contracts and creates a cleavage furrow, a barrier between the daughter cells (Cao and Wang, 1990). During abscission, the furrow closes to finally separate the daughter cells completely (Mierzwa and Gerlich, 2014).

The position of the furrow is defined by the location of the mitotic spindle during anaphase (Oliferenko et al., 2009; von Dassow, 2009). For a common symmetric division, the spindle can be found at the cell centre and the pole-pole axis is placed parallel to the cell long axis. However, in some cases, the spindle is arranged asymmetrically leading to an asymmetric division, which is crucial for development (Connell et al., 2011; Grill et al., 2001; Knoblich, 2001; Reinsch and Gonczy, 1998). 


\subsubsection{The function of ESCO2 in cell division and viability}

For correct transmission of genetic material during mitosis, chromosomes have to be replicated once followed by symmetric distribution between the daughter cells. Cohesin is a multi-subunit protein complex crucial for this process. Its core subunits, SMC1A, SMC3, RAD21/SCC1 and SCC3 build a ring-like structure (Nasmyth and Haering, 2009). As the name implies, cohesin is responsible for cohesion, which means to hold the sister chromatids together until they are symmetrically distributed to the two different daughter cells in metaphase (Onn et al., 2008).

In yeast, the acetyltransferase ECO1 (establishment of cohesion 1) is required for tethering of sister chromatids (Toth et al., 1999). ECO1 acetylates SMC3 at the positions K112 and K113 and is crucial for viability in yeast (Brands and Skibbens, 2005; Zhang et al., 2008).

Two ECO1 orthologues were identified in mammals, ESCO1 and ESCO2 (establishment of sister chromatid cohesion $\mathrm{N}$-acetyltransferase 1 and 2) (Bellows et al., 2003; Hou and Zou, 2005). A H2C2 zinc finger motif and an acetyltransferase domain make up the conserved C-terminal domain of both proteins (Hou and Zou, 2005). The N-terminal domain is divergent and crucial for chromosome binding (Hou and Zou, 2005). Both, ESCO1 and ESCO2, acetylate SMC3 since only loss of both enzymes decreased SMC3 acetylation (Nishiyama et al., 2010). However, both acetylases are functionally not redundant as shown in knockout mice and in human cells. In mice, ESCO2 deficiency with normal ESCO1 caused termination of development leading to early embryonic death (Whelan et al., 2012b). A defect of sister chromatid cohesion was observed causing a prometaphase delay and apoptosis (Whelan et al., 2012b). In human cells, loss of either enzyme, ESCO1 or ESCO2, led to an increase of defective cohesion (Hou and Zou, 2005).

Altogether, the non-redundant function of ESCO2 in chromatid cohesion is essential for cell viability and organism development. The important role of ESCO2 is further reflected in human diseases.

\subsection{Role of ESCO2 in human malformation syndromes}

Two diseases are known to be caused by mutations of ESCO2: Roberts syndrome and JubergHayward syndrome.

\subsubsection{Roberts syndrome (RBS)}

Roberts syndrome (RBS) is a disorder transmitted autosomal recessively and defined by microcephaly, pre- and postnatal growth retardation, cleft lip/palate, and either absence of all four limbs (tetraphocomelia) or severe limb reduction often accompanied with digit malformations (ectrodactyly and syndactyly) (Freeman et al., 1974; Van Den Berg and Francke, 1993). Besides, other abnormalities, like cardiac defects or corneal opacities leading to loss of vision, are common (Vega et al., 2010). Severely affected individuals often die during pregnancy or shortly after birth, whereas individuals with a milder form of RBS, also known as SC phocomelia, usually reach adulthood (Schule et al., 2005; Vega et al., 2010). The degree of cognitive impairment varies distinctly (Vega et al., 2010). 
In 2005, Esco2 was identified as the causative gene for RBS (Vega et al., 2005). So far, 30 different mutations in Esco2 were identified in RBS patients (Gordillo et al., 2008; Mengen et al., 2018; Resta et al., 2006; Schneeberger et al., 2020; Schule et al., 2005; Vega et al., 2010; Vega et al., 2005). Most of the identified mutations are truncating mutations leading to at least partial loss of the acetyltransferase domain (Gordillo et al., 2008; Vega et al., 2010). Small deletions or insertions shift the reading frame and create premature stop codons resulting in truncated proteins or mRNA instability as the transcripts may be degraded in nonsensemediated mRNA decay (Gordillo et al., 2008). Besides, mutations in introns were detected leading to aberrant splicing, which finally also results in a truncated protein or reduced mRNA amount (Gordillo et al., 2008; Schneeberger et al., 2020). One missense mutation was further investigated, which did not lead to a truncated protein and also levels of mRNA and protein were normal (Gordillo et al., 2008). A single amino acid of the acetyltransferase domain was changed resulting in a reduction of enzymatic activity. As the resulting cellular phenotype was undistinguishable to the other mutations, the authors concluded that the loss of ESCO2 acetyltransferase activity contributes to the pathogenesis of RBS (Gordillo et al., 2008).

No clear phenotype-genotype correlation was found, as different clinical severities did not occur with different kinds of mutations (Schule et al., 2005). However, some mutations correlate with the presence of corneal opacities, which in turn is statistically associated with the occurrence of mental retardation and cardiac abnormalities (Vega et al., 2010).

In a karyogram showing the metaphase chromosomes of affected cells, the lack of cohesion becomes visible as the centromeres separate prematurely, also known as heterochromatin repulsion or puffing (Freeman et al., 1974; German, 1979; Van Den Berg and Francke, 1993). These separation defects often results in sporadic aneuploidy (abnormal number of chromosomes) in the affected cells (Jabs et al., 1989; Schulz et al., 2008). Furthermore, increased cell death was found in RBS cells, which leads to a decreased proliferation capacity and was suggested to be responsible for the malformations observed in RBS (Gordillo et al., 2008).

\subsubsection{Juberg-Hayward syndrome (JHS)}

Juberg-Hayward syndrome (JHS) is an autosomal recessive malformation disorder, marked by microcephaly, cleft lip/palate, short stature, ptosis (dropping/falling of the upper eye lid), digit malformations (hypoplastic or aplastic thumbs, clinodactyly) and elbow restrictions caused by misplacement of the radial head or fusion of the forearm bones (Juberg and Hayward, 1969; Kantaputra and Mongkolchaisup, 1999; Kingston et al., 1982; Nevin et al., 1981; Verloes et al., 1992).

So far, three JHS patients were reported to be homozygous for the same mutation in Esco2 inducing a premature stop codon, which leads to truncation of the 50 C-terminal amino acids of the protein affecting the acetylase domain (Kantaputra et al., 2020a; Kantaputra et al., 2020b). As both disorders, RBS and JHS, are caused by ESCO2 mutations, they are called allelic. Interestingly, the disorders share several features, including microcephaly, cleft lip/palate, short stature, limb truncation, hypoplastic or aplastic thumbs, clinodactyly and elbow flexion contracture (Kantaputra et al., 2020b). In general, the symptoms of JHS are milder than in RBS. However, RBS and JHS are still considered as two different disorders since they have 
not been reported within the same families. Besides, the mutation causing JHS is not found in patients with RBS. Thus, a phenotype-genotype correlation was suggested (Kantaputra et al., 2020a; Kantaputra et al., 2020b).

In their recent papers, Kantaputra et al suggest explanations for the relatively mild phenotype of JHS compared to RBS, although in both disorders, the ESCO2 mutations interfere with its acetyltransferase activity (Kantaputra et al., 2020a; Kantaputra et al., 2020b). The Arg552Ter mutation generates the stop codon-containing sequence UGACUG, which was found to have a high read-through rate meaning that spontaneous read-through events might lead to a full-length protein (Cridge et al., 2018; Kantaputra et al., 2020a). However, in mammalian cell culture, the Arg552Ter mutation resulted in only a truncated protein and no full-length protein was detected in Western Blot (Kantaputra et al., 2020b). In another explanation, the remaining truncated protein may still fulfill non-acetyltransferase functions of the N-terminal domain to a higher extent than in RBS (Kantaputra et al., 2020b). Furthermore, expression of other genes may affect the severity of symptoms, which could also explain the phenotypic variability seen in individuals with the same ESCO2 mutation (Kantaputra et al., 2020b).

\subsection{The exosome complex regulates gene expression post-transcriptionally}

\subsubsection{Regulation of gene expression}

The genetic material of the somatic cells from one organism is equal but the tasks those various types of cells have to fulfil are highly diverse, making regulation of gene expression indispensable. The regulation occurs on different levels. Divers mechanisms regulate how much RNA is transcribed from a gene, how much of this is translated into protein and how long this protein is stable and how active it is.

Several mechanisms are known to regulate gene expression already on the level of DNA. DNA methylation, histone modifications and chromatin modifications determine which genes will be transcribed into RNA as they influence the accessibility of regulatory elements to transcription factors. Transcription factors bind to DNA sequences specifically and can promote or repress the transcription of a gene. Some transcription factors are master regulators in cortical development. Frequently, their expression is specific to a certain cell type. For example, PAX6 is a typical marker for RGCs. Several studies showed that PAX6 is essential for neurogenesis (Estivill-Torrus et al., 2002; Heins et al., 2002; Muzio et al., 2002). As the cells migrate into the SVZ, they progressively loose PAX6 and start to express TBR2 (also known as EOMES), which is associated with IPC identity (Englund et al., 2005).

One example for chromatin modifiers, are the ATP-dependent BAF (SWI/SNF) complexes. BAF complexes utilize ATP to change highly condensed heterochromatin into accessible euchromatin. By controlling chromatin state, BAF complexes regulate global gene expression programs (Narayanan et al., 2015). They consist out of at least 15 different subunits, including invariant core subunits (BAF47, BAF155, BAF170), two interchangeable core ATPase subunits (Brg1 or Brm), and lineage-specific subunits (Lessard et al., 2007). By the combination of different subunits, hundreds of different BAF complexes can be formed (Ronan et al., 2013), specific for e.g. neural progenitors, neurons, oligodendrocytes or Schwann cells. 
These complexes are crucial for cell-type specific gene expression (Narayanan and Tuoc, 2014). By this, BAF complexes regulate the differentiation of neural progenitor cells into distinct neuronal subtypes (Narayanan and Tuoc, 2014). BAF complexes are known to be essential for controlling gene transcription; however, it has not been investigated yet whether BAF complexes are important for post-transcriptional regulation as well.

Post-transcriptional mechanisms control the amount of protein translated from RNA via regulating RNA stability, splicing and transport. RNA can be modified by the addition of a certain chemical group, for example a methyl or acetyl group, to a specific position on the RNA. Proteins that transfer such a chemical group onto RNA are called writer proteins. Reader proteins can bind RNA and recognize these modifications specifically, which, in turn, can be removed by eraser proteins (Yang et al., 2018).

The most investigated RNA modification is the methylation of nitrogen 6 in adenosine (m6A). The m6A writer complex consists of RBM15, WTAP, METTL3 and METTL14 and can add methyl groups to RNA (Liu et al., 2014; Wang et al., 2016). Being an m6A eraser, Alkbh5 detaches these methyl groups (Zheng et al., 2013). YTH proteins and eIF3 function as m6A readers to detect m6A (Meyer et al., 2015; Patil et al., 2016; Wang et al., 2014). Recent studies indicate essential functions of m6A methylation in the control of RNA stability in the development of the brain (Flamand and Meyer, 2019; Wang et al., 2018; Widagdo and Anggono, 2018; Yoon et al., 2017). Notably, m6A was reported to affect not only RNA stability but also translation, splicing and transport (Dominissini et al., 2012; Kasowitz et al., 2018; Meyer et al., 2012; Wang et al., 2015; Xiao et al., 2016).

Next to m6A, about 170 other types of RNA modifications have been reported (Boccaletto et al., 2018; Helm and Motorin, 2017; Nachtergaele and He, 2018). For example, several studies showed that N6,2'-O-dimethyladenosine (m6Am), pseudouridine ( $\Psi)$, 5methylcytidine (m5C), N4-acetylcytidine (ac4C) and 8-oxo-7,8-dihydroguanosine (8-oxoG) affect stability of RNA (reviewed by Boo and Kim, 2020).

Enzymes that degrade RNAs are called ribonucleases and can be divided into three classes; endonucleases, which cut the RNA internally, and 3' and 5' exonucleases, which hydrolyse RNA from the 3' or 5' end, respectably (Houseley and Tollervey, 2009). The 3' exonuclease EXOSC10 and DIS3, which possess endonuclease and 3' exonuclease activity, belong to the exosome complex, the main eukaryotic ribonuclease complex, which is further described in the next section. The other group of ribonucleases in eukaryotic cells are the XRN exonucleases (Tatosyan et al., 2020). XRN exonucleases are the main 5' exonucleases and include the cytoplasmic XRN1 and nuclear XRN2, whose functions in general RNA turnover, rRNA maturation, nonsense-mediated decay (NMD) and termination of transcription have been shown to be indispensable in various species (Nagarajan et al., 2013).

Post-translational mechanisms control the activity and stability of proteins. For instance, small molecules, like amino acids or nucleotides, can bind to a protein and thereby change its conformation (Fischer et al., 2015). This conformational change can affect a protein function. Similarly, also protein-protein interactions can lead to a conformational change (Goh et al., 2004). This type of regulation is most obvious in protein complexes in which structural subunits modify the function of the catalytic subunits. Besides, also protein phosphorylation can alter protein function (Cohen, 1982; Rubin and Rosen, 1975; Ubersax and Ferrell, 2007). Protein 
kinases typically transfer a phosphate group from ATP to a hydroxyl group of a serine, threonine or tyrosine residue (Hunter, 1995; Rubin and Rosen, 1975). Protein phosphatases can reverse this process by hydrolysis of a phosphorylated residue (Hunter, 1995; Rubin and Rosen, 1975).

Two major protein degradation pathways control the stability of proteins, the ubiquitinproteasome pathway and lysosomal proteolysis. The addition of multiple ubiquitins targets proteins for subsequent degradation. The proteasome detects and degrades these polyubiquinated proteins (Callis, 1995; Hochstrasser, 1995; Koepp, 2014). Lysosomes are cellular organelles containing proteases and other digestive enzymes. Lysosomes take up proteins for degradation by the fusion with autophagosomes, vesicles formed by the enclosure of small areas of the cytoplasm in membranes originated from the endoplasmic reticulum (Bohley and Seglen, 1992; Huber and Teis, 2016).

\subsubsection{The exosome complex composition and its subunits}

The evolutionary conserved RNA exosome complex is a crucial modulator of gene expression in developmental processes (Januszyk and Lima, 2014; Kilchert et al., 2016). Even though, an exosome complex core with a similar architecture is also found in bacteria and archaea, the complex composition described here refers to the eukaryotic exosome complex. The exosome complex is structured in a ring-like fashion with eleven different subunits, including nine structural subunits (EXOSC1-9) and two catalytic subunits (EXOSC10, DIS3) (reviewed by Januszyk and Lima, 2014; Kilchert et al., 2016; Liu et al., 2006).

The structural subunits build up a core consisting out of a cap formed by EXOSC1-3 and a hexameric ring structure formed by heterodimers of EXOSC4-9 (Liu et al., 2006; Lorentzen et al., 2005; Makino et al., 2013). The trimeric cap subunits EXOSC1-3 carry S1 and/or KH domains facilitating RNA binding (Allmang et al., 1999; Aloy et al., 2002; Januszyk and Lima, 2010; Symmons et al., 2000). The ring subunits EXOSC4-9 harbor PH1 and/or PH2 domains mediating protein-protein binding and thereby carrying out an essential structural role (Liu et al., 2006; Schaeffer et al., 2009). Additionally, the PH domains were also shown to mediate sequence-specific RNA binding (Anderson et al., 2006). In connection with the structural elements, EXOSC10 and DIS3 can break down various RNAs using their ribonuclease activity. Both are functioning as exonucleases with DIS3 harboring an additional endonuclease activity (Lebreton et al., 2008; Lorentzen et al., 2008; Schaeffer et al., 2009; Schneider et al., 2009). Next to its exoribonuclease domain (EXO), EXOSC10 also holds a PMC2NT (polycystin $2 \mathrm{~N}$-terminal) domain, which interacts with the nucleic acid binding protein C1D, and a helicase and HRDC (RNase D C-terminal) domain than can bind RNA (Liu et al., 1999; Stead et al., 2007). The catalytically active domains of DIS3 are the RNB (ribonuclease B) and the PIN (PiLT N-terminal) domain carrying the endonuclease activity (Frazao et al., 2006; Mian, 1997; Schneider et al., 2009). Additionally, DIS3 contains the functionally important CR3 motif, two cold shock domains and a C-terminal S1 domain (Frazao et al., 2006; Schaeffer et al., 2012).

Different names are in use for the subunits of the eukaryotic exosome complex, for example, EXOSC10 is also known as RRP6 (Ribosomal RNA-processing protein 6) and DIS3 
as RRP44, which originate from the terms of the yeast genes. Here, I use the names from the human homologs.

The exosome complex conducts RNA processing and decay in the cytoplasm and the nucleus, including the nucleolus (Butler and Mitchell, 2011; Schaeffer et al., 2011; Schneider and Tollervey, 2013). The cytoplasmic exosome consists out of the 9 subunit core and DIS3, the nucleolar exosome contains EXOSC10 instead of DIS3 and in the nucleus both nucleases are associated with the core (Januszyk and Lima, 2014; Tomecki et al., 2010). In humans, three versions of DIS3 were identified, named hDIS3, hDIS3L and hDIS3L2 (Malecki et al., 2013; Staals et al., 2010; Tomecki et al., 2010). hDIS3 associates with the exosome complex core in the nucleus together with EXOSC10, whereas DIS3L is the catalytic subunit of the cytoplasmic exosome complex (Tomecki et al., 2010). DIS3L2 is found in the cytoplasm without connection to the exosome complex (Malecki et al., 2013).

In the nucleus, DIS3 and EXOSC10 appear to be co-dependent since EXOSC10 has been shown to stimulate DIS3 exo- and endonucleolytic activity and EXOSC10 activity relies on DIS3 exonucleolytic activity (Gudipati et al., 2012; Schneider et al., 2012). Additionally, the exo- and endonucleolytic activities are also dependent on the core (Wasmuth and Lima, 2012).

The cytoplasmic, the nuclear and the nucleolar complexes associate with different sets of cofactors. The structural subunits interact with their cofactors through composite surfaces enabling the exosome complex to target specific RNAs. Thereby, the subunits deliver functional specificity (Januszyk and Lima, 2014; Lubas et al., 2011). Hence, the entire exosome complex is indispensable for regulating RNA quantities and eliminating defective RNAs.

\subsubsection{The exosome complex functions and its cofactors}

The exosome complex is responsible for general mRNA degradation and thereby regulating protein-synthesis rates. For instance, in human cells, the exosome complex is recruited to transcripts harboring AU-rich sequence elements (AREs) (Chen et al., 2001; Kilchert et al., 2016; Mukherjee et al., 2002; Tran et al., 2004). These are present in short-lived human mRNAs coding for proteins with transient expression, including growth factors and proto-oncogenes. An important cofactor for cytoplasmic mRNA degradation by the exosome complex is the SKI (Superkiller) complex formed by SKI2, SKI3 and SKI8 (Anderson and Parker, 1998). Their human homologs are SKIV2L, TTC37 and WDR61, respectively. SKI2 is an RNA helicase and SKI3 and SKI8 contain structural motifs known to be relevant for protein-protein interactions (Halbach et al., 2013). In yeast, the SKI complex and the exosome complex were shown to form a joint continuous channel directing the RNA from the helicase to the nuclease (Halbach et al., 2013).

The exosome complex is also part of RNA quality control by breaking down improperly processed or defective mRNAs. The exosome complex performs nonsense-mediated decay (NMD) by the turnover of transcripts carrying premature termination codons, nonstop decay (NSD) by degrading transcripts missing termination codons and no-go-decay (NGD) by the decay of transcripts in which translation has stalled (Doma and Parker, 2006; Houseley et al., 
2006; Lejeune et al., 2003; Mitchell and Tollervey, 2003; Schaeffer et al., 2011; Takahashi et al., 2003; van Hoof et al., 2002).

Despite degrading mRNA in the cytoplasm, the exosome complex degrades pre-mRNAs already in the nucleus. For example, also inefficiently spliced pre-mRNA is degraded by the exosome complex (Kilchert et al., 2015). The transcripts may retain "decay-promoting" introns containing sequences that promote binding of the exosome specificity factor Mmi1 inducing degradation by the exosome complex. Fast splicing of introns harboring Mmi1-binding sites prevents the decay. Increased intron retention resulting in low gene expression was shown to be a response to stress in yeast (Kilchert et al., 2015).

The exosome complex also processes noncoding RNAs (ncRNAs). A crucial function in the processing of ribosomal RNA (rRNA) was reported already in the nineties showing that the exosome complex is required for 3' processing of 7S pre-rRNA to 5.8S rRNA (Mitchell et al., 1997; Mitchell et al., 1996). The exosome complex was also found to be involved in the maturation and surveillance of small nuclear RNA (snRNA) and small nucleolar RNA (snoRNA) (Allmang et al., 1999; van Hoof et al., 2000). Defective precursors of transfer RNA (tRNA), ribosomal RNA (rRNA), snRNA and snoRNA are targeted and polyadenylated by the TRAMP (TRF4/5-AIR1/2-MTR4 Polyadenylation) complex, which then activates the exosome complex (Kadaba et al., 2004; Kadaba et al., 2006; Schneider et al., 2012; Vanacova et al., 2005). The TRAMP complex consists out of the poly(A)-polymerase TRF4 or TRF5, the RNA helicase MTR4 and the RNA-binding proteins AIR1 and AIR2 and is a key nuclear cofactor of the exosome complex as it polyadenylates transcripts to facilitate the hydrolysis by the exosome complex (Butler and Mitchell, 2011; LaCava et al., 2005; Vanacova et al., 2005). Besides, the exosome complex also breaks down cryptic unstable transcripts (CUTs), which belong to the group of unstable ncRNAs (Schneider et al., 2012; Wyers et al., 2005). Another important nuclear cofactor of the exosome complex is the NEXT (Nuclear Exosome Targeting) complex, involving the RNA helicase MTR4 together with the RNA-binding protein RBM7 and the Zincknuckle containing protein ZCCHC8, which are suggested to be essential for substrate targeting (Lubas et al., 2011). The NEXT complex was shown to be required for the degradation of promoter upstream transcripts (PROMPTs) by the exosome complex (Lubas et al., 2011). PROMPTs are noncoding RNAs found upstream of transcription start sites (Preker et al., 2008). Their general functions remain uncertain; however, roles in methylation and regulatory purposes were described (Imamura et al., 2004; Lloret-Llinares et al., 2016; Preker et al., 2008).

All in all, the exosome complex is essential for post-transcriptional processing and decay of different RNA types.

\subsection{Role of the exosome complex in neurodevelopmental disorders}

Mutations in several exosome subunits have been linked a variety of syndromes affecting different tissues including the nervous system. In particular, EXOSC2 mutations have been associated to the SHRF (short stature, progressive hearing loss, retinitis pigmentosa and distinctive facies) syndrome (Di Donato et al., 2016). Various mutations in EXOSC3, EXOSC8 and EXOSC9 were identified in patients affected by different types pontocerebellar hypoplasia (PCH) (Boczonadi et al., 2014; Burns et al., 2018; Wan et al., 2012). Individuals with mutations in EXOSC10 display microcephaly and a global developmental delay often accompanied with 
motor problems. Mutations in DIS3 have been associated to multiple myeloma, a malignancy of mature B-lymphoid cells in the bone marrow (Chapman et al., 2011; Weissbach et al., 2015). The syndromes with neurodevelopmental contribution are discussed in more detail below and are summarized in Table 1.

\begin{tabular}{|c|c|c|c|c|c|c|}
\hline $\begin{array}{l}\text { Gene } \\
\text { name }\end{array}$ & $\begin{array}{l}\text { Exosome } \\
\text { subunit }\end{array}$ & Mutations & Genotypes & $\begin{array}{c}\text { Human } \\
\text { disorders }\end{array}$ & Core phenotypes & References \\
\hline & & G30V & G30V hom & & & \\
\hline RRP4 & EXOSC2 & G198A & G30V/G198A & SHRF syndrome & $\begin{array}{l}\text { short stature; progressive hearing loss; retinitis } \\
\text { pigmentosa; distinctive facies; premature } \\
\text { ageing; mild intellectual disability }\end{array}$ & $\begin{array}{l}\text { (Di Donato et al., } \\
\text { 2016) }\end{array}$ \\
\hline \multirow{10}{*}{ RRP40 } & \multirow{10}{*}{ EXOSC3 } & G31A & G31A hom & \multirow{9}{*}{$\begin{array}{l}\text { Pontocerebellar } \\
\text { hypoplasia type } \\
1 \mathrm{~b}(\mathrm{PCH} 1 \mathrm{~b})\end{array}$} & \multirow{9}{*}{$\begin{array}{c}\text { atrophy of pons and cerebellum; microcephaly; } \\
\text { severe developmental delay; muscle atrophy } \\
\text { and weakness; respiratory impairment }\end{array}$} & \multirow{9}{*}{$\begin{array}{c}\text { (Biancheri et al., } \\
\text { 2013; Di } \\
\text { Giovambattista et } \\
\text { al., 2017; Eggens et } \\
\text { al., 2014; Ivanov et } \\
\text { al., 2018; Le Duc et } \\
\text { al., 2020; Rudnik- } \\
\text { Schoneborn et al., } \\
\text { 2013; Saugier-Vebe }\end{array}$} \\
\hline & & V80F & G31A/W238R & & & \\
\hline & & Y109N & Y109N/D132A & & & \\
\hline & & D132A & D132A hom & & & \\
\hline & & G135E & D132A/A139P & & & \\
\hline & & A139P & D132A/G191C & & & \\
\hline & & G191C & D132A/null & & & \\
\hline & & \multirow{3}{*}{$\begin{array}{c}\text { W238R } \\
+ \text { various null } \\
\text { mutations }\end{array}$} & G135E hom & & & \\
\hline & & & & & & \\
\hline & & & $\begin{array}{r}\text { G191C hom } \\
\text { V80F/D132A }\end{array}$ & \begin{tabular}{|c|}
$\begin{array}{c}\text { Hereditary } \\
\text { spastic paraplegia } \\
\text { (HSP) }\end{array}$ \\
\end{tabular} & $\begin{array}{l}\text { progressive spastic paraplegia; cerebellar } \\
\text { atrophy; strabismus; mild cognitive symptoms }\end{array}$ & $\begin{array}{l}\text { (Halevy et al., 2014; } \\
\text { Zanni et al., 2013). }\end{array}$ \\
\hline & & $\mathrm{A} 2 \mathrm{~V}$ & A2V hom & & cerebellar and corpus callosum hypoplasia; & \\
\hline RRP43 & EXOSC8 & S272T & S272T hom & $\mathrm{PCH} 1 \mathrm{c}$ & $\begin{array}{c}\text { hypomyelination; spinal muscular atrophy } \\
\text { (SMA); severe muscle weaknes; psychomotor } \\
\text { deficit; respiratory impairment }\end{array}$ & $\begin{array}{l}\text { (Boczonadi et al., } \\
\text { 2014) }\end{array}$ \\
\hline \multirow{5}{*}{ RRP45 } & \multirow{5}{*}{ EXOSC9 } & L14P & L14P hom & \multirow{5}{*}{ PCH1d } & \multirow{5}{*}{$\begin{array}{c}\text { progressive SMA-like motor neuronopathy; } \\
\text { cerebellar atrophy with normal pons; } \\
\text { progressive muscle weakness; severe } \\
\text { developmental delay; sometimes respiratory } \\
\text { impairment }\end{array}$} & \multirow{5}{*}{$\begin{array}{l}\text { (Bizzari et al., 2020; } \\
\text { Burns et al., 2018; } \\
\text { Sakamoto et al., } \\
\text { 2020) }\end{array}$} \\
\hline & & G51R & L14P/R161X & & & \\
\hline & & L80R & G51R hom & & & \\
\hline & & R161X & L80R/R162KfsX3 & & & \\
\hline & & R162KfsX3 & & & & \\
\hline \multirow{7}{*}{ RRP6 } & \multirow{7}{*}{ EXOSC10 } & P54L & \multirow{7}{*}{$\begin{array}{l}\text { all heterozygous } \\
\text { with only one } \\
\text { disease allele }\end{array}$} & \multirow{7}{*}{$\begin{array}{l}\text { Novel syndrome } \\
\text { with } \\
\text { microcephaly }\end{array}$} & \multirow{7}{*}{$\begin{array}{l}\text { microcephaly; global developmental delay; } \\
\text { often motor symptoms }\end{array}$} & \multirow{7}{*}{$\begin{array}{l}\text { (Ulmke et al., in } \\
\text { reparation) }\end{array}$} \\
\hline & & $\mathrm{D} 295 \mathrm{~A}$ & & & & \\
\hline & & S321T & & & & \\
\hline & & Q330H & & & & \\
\hline & & G420Pfs & & & & \\
\hline & & G459S & & & & \\
\hline & & V568M & & & & \\
\hline
\end{tabular}

\subsubsection{Pontocerebellar hypoplasia $(\mathrm{PCH})$}

Pontocerebellar hypoplasia $(\mathrm{PCH})$ is a clinically and genetically diverse group of rare neurodegenerative diseases defined by progressive atrophy of various parts of the brain and a global developmental delay (Namavar et al., 2011). Various types of PCH caused by different gene mutations have been reported. $\mathrm{PCH}$ types $1 \mathrm{~b}, 1 \mathrm{c}$ and $1 \mathrm{~d}$ have been linked to mutations of exosome complex subunits. 


\section{PCH1b}

In PCH1b, pontine and cerebellar atrophy are observed starting at birth together with abnormalities of Purkinje cells and degeneration of spinal motor neurons, which manifests in microcephaly, developmental delay and muscle atrophy and weakness (Wan et al., 2012). More than $60 \mathrm{PCH} 1 \mathrm{~b}$ patients with an EXOSC3 mutation were described. Thereby, several pathogenic missense variants of EXOSC3 in which one amino acid is exchanged were found: G31A, V80F, Y109N, D132A, G135E, A139P, G191C and W238R (Biancheri et al., 2013; Di Giovambattista et al., 2017; Eggens et al., 2014; Ivanov et al., 2018; Le Duc et al., 2020; Rudnik-Schoneborn et al., 2013; Saugier-Veber et al., 2020; Schottmann et al., 2017; Schwabova et al., 2013; Wan et al., 2012). PCH1b patients were reported to carry missense variants in homozygous condition or in combination with another pathogenic variant (compound heterozygous condition). This second pathogenic variant can be either another missense variant or a null mutation meaning a loss-of-function mutation, e.g. by a frameshift or whole exon deletions (Eggens et al., 2014; Rudnik-Schoneborn et al., 2013). Heterozygous individuals with one healthy allele were not affected (Rudnik-Schoneborn et al., 2013). Complete loss-of-function of EXOSC3 by a homozygous null mutation was not observed and therefore suggested to be embryonically lethal (Rudnik-Schoneborn et al., 2013).

The different EXOSC3 variants and their different combinations observed vary in the disease severity, mainly defined by the age of death. For example, individuals homozygous for the G31A variant were reported to have a life expectancy between 4 days to 17 months, whereas D132A homozygous individuals had a lifespan of 5 to 18 years (Biancheri et al., 2013; Eggens et al., 2014; Ivanov et al., 2018; Rudnik-Schoneborn et al., 2013; Schottmann et al., 2017; Schwabova et al., 2013; Wan et al., 2012). Besides, the variant combinations V80F/D132A and homozygous G191C were only linked to hereditary spastic paraplesia without typical PCH1b symptoms (discussed later in this section). Thus, a genotype-phenotype correlation was suggested (Eggens et al., 2014; Rudnik-Schoneborn et al., 2013).

However, also for the same pathogenic variant composition, the clinical manifestations may differ. For instance, the combination of D132A and G191 variants was reported in two siblings exhibiting different severities of PCH1b with one sibling showing a prenatal-onset of disease and never reaching developmental milestones and the other with a postnatal disease onset and delayed psychomotor development but still reaching all milestones (Le Duc et al., 2020). The first sibling was the only one displaying hypotonia and muscle atrophy. Further, the cognitive impairment varied from severe intellectual disability in the first sibling to merely learning disabilities in the other one. Another example for clinical variations is the G31A homozygous genotype. Previous studies associated G31A homozygosity with a severe PCH1b phenotype (Di Giovambattista et al., 2017; Eggens et al., 2014; Rudnik-Schoneborn et al., 2013; Schwabova et al., 2013; Wan et al., 2012). More recently, G31A homozygous patients were described with aspinal muscular atrophy (SMA)-like phenotype lacking hypoplasia of pons and cerebellum in the first months of life (Ivanov et al., 2018). Besides, two G31A homozygous fetuses have been reported displaying microlissencephaly (microcephaly with a smooth brain surface) and rhombencephalosynapsie (malformation of the cerebellum with agenisis of the vermis and fusion of cerebellar hemispheres), which were not mentioned with PCH1 before (Saugier-Veber et al., 2020). These studies cast doubt on the proposed clear genotypephenotype correlation. Instead, other factors were suggested to be involved in the resulting 
phenotype. The individual genetic architecture could influence the course of disease. Additional genetic variants missed in exome sequencing may cause a more severe phenotype or variants crucial for maintenance of cellular functions could contribute to a milder phenotype (Le Duc et al., 2020). In addition, epigenetic alterations and the gender could have an impact (Le Duc et al., 2020).

Studies in model organisms further support the finding that EXOSC3 missense variants identified in PCH1b patients diminish the function of the exosome complex. Depletion of EXOSC3 in zebrafish embryos leads to hindbrain shrinkage, which can be rescued by wildtype zebrafish EXOSC3 mRNA (Wan et al., 2012). However, mutant zebrafish EXOSC3 mRNA resembling the human variants G31A, D132A and W238R, could not improve the hindbrain defect (Wan et al., 2012). In addition, in mouse neuronal cell line, the expression levels of mouse EXOSC3 mRNA corresponding to the G31A and W237R variants were lower than wild-type EXOSC3 expression levels (Fasken et al., 2017). These finding suggests that the amino acid alterations interfere with folding of the EXOSC3 protein.

Investigations of the exosome complex structure indicate that the G31, D132, and W238 residues of EXOSC3 might be relevant for interactions with EXOSC5 and EXOSC9 (Fasken et al., 2017). The G31 residue in the N-terminal domain of EXOSC3 is tightly wrapped close to EXOSC5. Thus, the G31A variation could affect the association with EXOSC5 (Fasken et al., 2017). The D132 residue is positioned in a loop between strands of the EXOSC3 S1 domain. Hence, the D132A variation might hamper folding of the loop and interfere with interactions of EXOSC3 with EXOSC5 and EXOSC9 (Fasken et al., 2017). The W238 residue is located in a pocket between the $\mathrm{S} 1$ and $\mathrm{KH}$ domains and may be important for the correct positioning of residues to interact with EXOSC9. Thereby, the W238R mutation could impair interactions with EXOSC9 (Fasken et al., 2017). Altogether, the amino acid substitutions in EXOSC3 linked to $\mathrm{PCH} 1 \mathrm{~b}$ could impede interactions with other complex subunits, impairing exosome complex function.

\section{PCHIC}

22 individuals from three families diagnosed with $\mathrm{PCH} 1 \mathrm{c}$ were reported with a mutation in EXOSC8, characterized by cerebellar and hypoplasia of cerebellum and corpus callosum, spinal muscular atrophy (SMA) and hypomyelination starting at birth (Boczonadi et al., 2014). Clinically, the patients present psychomotor deficit, impaired vision and hearing and severe muscle weakness, typically leading to death in the first years by respiratory failure. Similar to PCH1b-affected individuals, PCH1c-affected patients show impairments in spinal motor neurons and Purkinje cells. Additionally, they display defects in oligodendroglia causing hypomyelination (Boczonadi et al., 2014).

Two different homozygous mutations of EXOSC8 were found in the affected individuals, both altering conserved amino acids (A2V and S272T) (Boczonadi et al., 2014). The A2V mutation is associated with a less severe PCH1c with a slightly longer lifespan of $\geq 2.3$ years instead of $\leq 2$ years in the $\mathrm{S} 272 \mathrm{~T}$ mutation.

Structural analysis revealed that the $\mathrm{S} 272$ residue is positioned in the $\mathrm{PH}$ domain and could be crucial for interaction with EXOSC9 or affect opening of the RNA channel (Boczonadi 
et al., 2014; Liu et al., 2006; Morton et al., 2018). The A2 residue could be relevant for the interaction with EXOSC1 or affect an opening located at the side of the exosome complex (Liu et al., 2006; Morton et al., 2018). Despite potentially affecting the interaction and function of EXOSC8, both mutations lead to a decreased amount of EXOSC8 protein (Boczonadi et al., 2014). Interestingly, in cells from EXOSC8 patients, also reduced levels of EXOSC3 protein were identified suggesting a close interaction between both exosome complex subunits (Boczonadi et al., 2014).

Upregulated mRNA levels of the ARE-containing myelin proteins MBP and MOBP were found in patient myoblasts (Boczonadi et al., 2014). These findings could be repeated in human oligodendroglia cells upon downregulation of EXOSC 8 by siRNA. The authors suggest that a disturbed balance between myelin components may cause the demyelinating disease. Similarly, defective myelination was described in EXOSC8 knockdown zebrafish (Boczonadi et al., 2014). An initial increase of myelin proteins was observed. The loss of oligodendroglia then lead to a decrease of myelin proteins later.

Additionally, in patient fibroblasts, SMN1 expression was increased, which is associated with SMA (Boczonadi et al., 2014). Notably, patient fibroblasts also show an increase of developmental HOX mRNA levels and the epigenetic regulator HOTAIR long ncRNA levels, similar to RBM7 mutant fibroblasts (Giunta et al., 2016). Levels of other ARE and non-ARE mRNAs were tested in patient cells as well as cells in which EXOSC8 was downregulated by siRNA. No general change in other mRNA levels was detected indicating only specific transcripts to be affected by EXOSC8 dysfunction (Boczonadi et al., 2014).

Taken together, the EXOSC8 mutation in PCH1c-affected patients seems to diminish exosome complex function, causing accumulation of specific transcripts, like HOX, SMN1 and myelin-related mRNAs, which finally impairs neurodevelopment and myelin synthesis.

\section{PCHId}

The disorder associated with EXOSC9 mutations was reported as PCH-like or PCH1d (Bizzari et al., 2020; Burns et al., 2018; Sakamoto et al., 2020).

Three missense mutations (L14P, G51R and L80R) and two truncating mutations, including one nonsense mutation (R161X) and a frameshift mutation leading to an early stop codon (R162KfsX3), were identified (Burns et al., 2018; Sakamoto et al., 2020). The L14 residue is placed in the first alpha helix of EXOSC9 as shown by structure analysis and the mutation could disturb intramolecular interactions of the protein (Fasken et al., 2020; Liu et al., 2006). The other missense mutations are within the first PH domain (Sakamoto et al., 2020). A disruption of the $\mathrm{PH}$ domain could interfere with the sequence-specific RNA binding of the exosome complex (Anderson et al., 2006).

So far, nine individuals were reported with an EXOSC9 mutation, either homozygous for a missense mutation or compound heterozygous with a missense and a truncating mutation. In particular, five patients have been revealed homozygous for the L14P variant, one heterozygous for L14P and R161X, one homozygous for G51R, two heterozygous for L80R and R162KfsX3 (Bizzari et al., 2020; Burns et al., 2018; Sakamoto et al., 2020). 
The affected individuals show a neurodegenerative disorder with early-onset, progressive SMA-like motor neuronopathy and atrophy of the cerebellum (Bizzari et al., 2020; Burns et al., 2018; Sakamoto et al., 2020). Similar to patients with EXOSC3 or EXOSC8 mutations, the affected individuals all show progressive muscle weakness and severe developmental delay. Some also show respiratory impairment (Burns et al., 2018). Unlike EXOSC3/EXOSC8 mutation patients, they display a relatively normal pons (Bizzari et al., 2020; Burns et al., 2018; Sakamoto et al., 2020). One individual with compound heterozygous EXOSC9 mutations (L14P and R161X) showed a more severe form and died of respiratory failure at 15 months, whereas the homozygous L14P patients were alive at the ages of 19 months, 28 months, 3 years, 4.5 years and 4 years and 7 months, respectively (Bizzari et al., 2020; Burns et al., 2018). Besides, this individual also showed congenital fractures of the long bones (Burns et al., 2018).

Individuals compound heterozygous for L80R and R162KfsX3 variants exhibit similar disease characteristics as the other patients with EXOSC9 variants, however, both individuals showed slight intellectual development, with one being able to read some words and the other to participate in a basic conversation (Sakamoto et al., 2020). The clinical difference between individuals harboring different missense variants of EXOSC9 indicates a genotype-phenotype correlation.

Patient derived fibroblasts homozygous for the L14P variant showed a reduction of EXOSC9 expression compared to controls (Burns et al., 2018). In skeletal muscle from the patient with heterozygous EXOSC9 mutations (L14P and R161X), protein levels were even lower matching the more severe phenotype (Burns et al., 2018). Interestingly, in patient fibroblasts expressing reduced levels of the EXOSC9-L14P variant, EXOSC3 and EXOSC8 levels were normal, whereas in cells with reduced levels of the EXOSC3-G31A or EXOSC8A2V variant, amounts of other exosome complex subunits were reduced (Burns et al., 2018). Hence, in contrast to the investigated mutations of EXOSC3 and EXOSC8, the L14P variant of EXOSC9 may not destabilize the whole exosome complex. However, the EXOSC9-L14P fibroblasts showed a reduced amount of EXOSC3 within the exosome complex (Burns et al., 2018). Thus, the EXOSC9-L14P variant seems to impair the exosome complex by interfering with the association of different subunits within the complex, potentially altering the formation of the entire complex (Burns et al., 2018; Fasken et al., 2020).

RNA sequencing analysis of patient derived fibroblasts homozygous for the EXOSC9L14P variant revealed upregulation of many ARE-containing transcripts (Burns et al., 2018). Many differently expressed mRNAs are associated with neuronal system development. Besides, levels of developmental HOXC8 mRNA were increased. In contrast to observations in EXOSC8 and RBM7 mutant cells, levels of the epigenetic regulator HOTAIR ncRNA levels were unchanged (Burns et al., 2018; Giunta et al., 2016). RNA sequencing of skeletal muscle from the individual with heterozygous EXOSC9 mutations (L14P and R161X) revealed changes in the levels of transcripts related to motor neuronopathy and bone disease (Burns et al., 2018). Comparison of RNA expression in fibroblasts and in muscle show a low overlap of differently expressed transcripts indicating tissue-specific roles of EXOSC9 mutations (Burns et al., 2018). 


\subsubsection{Hereditary spastic paraplegia (HSP)}

Hereditary spastic paraplegia (HSP) is a heterogeneous group of neurodegenerative motor system diseases defined by a progressive spasticity of the lower limps accompanied by diverse additional symptoms (Fink, 2003).

Four patients diagnosed with HSP were identified to carry a homozygous EXOSC3 mutation (G191C) (Halevy et al., 2014). The individuals show progressive spastic paraplegia with cerebellar atrophy and strabismus. The patients present signs of cerebellar dysfunction, like nystagmus (involuntary eye movement) or dysmetria (lack of coordination of movement). They also display variable but generally mild cognitive symptoms, including speech delay or attention deficit disorder. Typical symptoms for $\mathrm{PCH}$, like microcephaly, hypotonia, respiratory impairment, atrophy of the pons or defective myelination were not observed.

Two patients fitting into the wide range of HSP with delayed motor milestones, progressive spasticity and mild cognitive impairment were reported to be compound heterozygous for two EXOSC3 missense variants, V80F and D132A (Zanni et al., 2013). Similar as for the individuals with the G191C variant of EXOSC3, cerebellar atrophy was reported with preserved brainstem and pons. In contrast to the others, V80F/D132A compound heterozygous patients also showed indications of lower motor neuron dysfunction, like tongue atrophy and fasciculations.

These studies indicate that EXOSC 3 mutations cannot only cause a PCH1 phenotype, but also a form of HSP without pontine hypoplasia or atrophy.

\subsubsection{SHRF syndrome}

Three individuals were reported with a novel syndrome characterized by a short stature, progressive hearing loss, retinitis pigmentosa and distinctive facies (SHRF). Besides the patients also displayed premature skin ageing and mild intellectual impairment. Whole exome sequencing demonstrated homozygous (Gly30Val) or compound heterozygous (Gly30Val and G198A) missense mutations of the gene coding for EXOSC2 (Di Donato et al., 2016).

Structural modeling of the exosome complex structure suggests that the G30V substitution in EXOSC2 could weaken interactions with EXOSC4 (Di Donato et al., 2016). The G198 residue is placed at the end of a $\beta$-strand in the KH domain of EXOSC2. The G198D variant may truncate and impair the $\beta$-hairpin structure and thereby interfere with the structure of EXOSC2 (Di Donato et al., 2016).

Comparing the phenotypes of the affected individuals to the disease caused by mutations of EXOSC3 or EXOSC8, only a minimal overlap of clinical features is detectable. In the EXOSC2 mutant individuals, only borderline or no atrophy of the cerebellum and no indications for neurodegeneration were observed, even though the highest level of EXOSC2 expression is found in the cerebellar hemispheres (Di Donato et al., 2016). Thus, the authors conclude that the loss of EXOSC2 function in neurons might be compensated by other subunits of the exosome complex, like EXOSC3, but not vice versa (Di Donato et al., 2016).

Next to cell-type-specific compensation mechanisms, also different substrate specifity conferred by different complex subunits can be a possible explanation of the striking differences 
in diseases caused by EXOSC2 or EXOSC3 mutations. Indeed, investigation of RRP4-G226D mutant yeast cells modelling the human EXOSC2-G198A variant revealed different RNA targets compared to a yeast model of the human EXOSC3-W238R variant associated with PCH1b (Sterrett, 2020). RRP4-G226D cells display a growth defect, which is worsened by the deletion of Mpp6, a cofactor of the exosome complex (Sterrett, 2020). This effect was not seen in RRP40-W195R cells modelling the human EXOSC3 mutation (Sterrett, 2020). Mpp6 stabilizes the interaction between the exosome complex and the RNA helicase MTR4 (Falk et al., 2017). Therefore, the authors suggest that the G226D amino acid substitution could cause a destabilization of the critical interaction of MTR4 and the exosome complex (Sterrett, 2020).

How the mutations impede exosome complex function, was further investigated in patient-derived lymphoblasts (Yang et al., 2020). The G198D but not G30V variation impairs EXOSC2 protein stability with EXOSC2-G198D having a reduced half-life. Further, interactions of EXOSC2 with other RNA exosome components were affected as shown by coimmunoprecipitation. Additionally, protein levels of EXOSC3 and EXOSC10 were decreased in G198D mutation-carrying samples compared to samples containing only the G30V mutation. Because SHRF patients show premature skin aging, the effect of EXOSC2 loss on primary human keratinocytes was investigated (Yang et al., 2020). Indeed, knockout of EXOSC2 resulted in a reduced proliferation rate. Whereas, wild-type EXOSC2 and EXOSC2-G30V could rescue the cell differentiation defect, transduction with EXOSC2-G198D variant could not. Thus, EXOSC2-G198V seems to be a non-functional protein linked to impaired cell proliferation, whereas EXOSC2-G30V is a weaker disease allele (Yang et al., 2020).

A EXOSC2 knockout drosophila model presented impairments in eye development and maintenance, and development of the muscle structure and wing veins (Yang et al., 2020). Further investigations showed that autophagy was impaired in patient cells, as well as in EXOSC2 knockout human keratinocytes and in EXOSC2 knockout drosophila model (Yang et al., 2020). In drosophila, overexpression of the transcription factor MITF or of the autophagy genes ATG1 and ATG17, controlled by MITF, rescued the eye defects and adult lethal phenotypes caused by EXOSC2 knockout (Yang et al., 2020). Rapamycin treatment also rescued the lethality in EXOSC2 knockout flies (Yang et al., 2020). Rapamycin inhibits the mTOR pathway, which regulates many processes including autophagy, and thereby augments mRNA turnover (Albig and Decker, 2001; Martinez-Nunez et al., 2017). Additionally, in RNA sequencing analysis of RRP4-G226D yeast cells, expression of several autophagy genes was decreased (Sterrett, 2020). Taken together, defective autophagy seems to play a role in SHRF pathogenesis.

The role of EXOSC2 was further investigated in EXOSC2 knockout zebrafish, which displayed microcephaly, retinis pigmentosa, loss of spinal motor neurons and myelin deficiency (Yatsuka et al., 2020). EXOSC2 knockout resulted in impaired mRNA turnover and an imbalance in the nucleotide pool (Yatsuka et al., 2020). Rapamycin treatment improved the nucleotide pool imbalance, prolonged survival and partially rescued the neuronal defects in EXOSC2 knockout zebrafish (Yatsuka et al., 2020). Notably no decrease of autophagy-related mRNA was found (Yatsuka et al., 2020). These results indicate that further examination on the role of rapamycin in RNA decay and EXOSC2 deficiency in autophagy is needed. 


\subsubsection{Novel syndrome with microcephaly}

A novel syndrome was associated with mutations in EXOSC10, an exonuclease of the exosome complex (Ulmke et al, in preparation). Seven affected individuals were identified so far, displaying microcephaly and global developmental delay, often accompanied with motor problems, like difficulties with walking and coordination. Cerebellar hypoplasia, a smaller corpus callosum and abnormal cortical gyration was found in some patients.

Whereas patients with PCH1b/c/d, HSP or SHRF carry a homozygous or compound heterozygous mutation of an exosome complex subunit, the individuals were identified to carry only one disease allele of EXOSC10. Hence, in contrast to the other diseases associated with exosome complex malfunction, the inheritance seems to be dominant. Six different missense mutations were found (Fig.1.2), with one in the N-terminal PMC2NT domain (P54L), which is proposed to interact with the nucleic acid binding protein C1D (RRP47) (Stead et al., 2007). The other missense mutations are all placed in the exoribonucleolytic domain (D295A, S321T, Q330H, G459S, V568M). One frameshift mutation was identified leading to premature termination (G420Pfs).

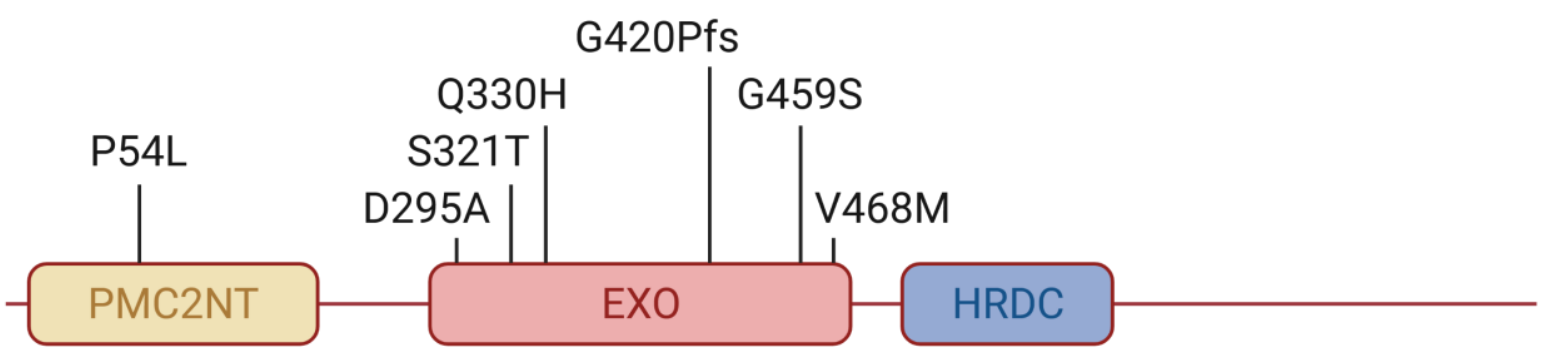

Figure 1.2 EXOSC10 and its mutations in patients. Illustration of EXOSC10 with its PMC2NT (polycystin 2 N-terminal) domain, EXO (exoribonuclease) domain and HRDC (RNase D C-terminal) domain. The identified mutations are indicated (six missense and one frameshift mutation) (unpublished data). Created with BioRender.com.

\subsection{General objectives of the studies}

The specific objectives of the studies presented in chapters 2 and 3 were aimed at identifying and investigating molecular mechanisms orchestrating the development of the mammalian cerebral cortex and to reveal the molecular mechanisms underlying cortical malformation.

In the first part of this study (Chapter 2), we decided to focus on IPCs as they are the progenitors of the vast majority of neurons in the cortex. Accordingly, mutations in IPC genes have been shown to cause malformations like microcephaly (Baala et al., 2007; Glickstein et al., 2009). Therefore, we purified IPCs from the developing mouse cortex and established the transcriptome profile of IPCs by RNA sequencing, indicating which genes are activated in IPCs specifically. Comparing these genes to literature, we identified factors, which are likely to control IPC genesis. We chose one of them, ESCO2, for further analysis.

Recent sequencing analyses of cortical malformations revealed a multifarious genetic landscape (Hu et al., 2014; Perenthaler et al., 2019). In our pilot work, we identified novel microcephaly-related mutations in a gene encoding EXOSC10, a core subunit of the RNAdecay exosome complex. In the second part of this work (Chapter 3), we characterized the cortical phenotypes of EXOSC10cKO mutants. 


\section{Chapter 2: Molecular profiling reveals involvement of ESCO2 in intermediate progenitor cell maintenance in the developing mouse cortex}

All elements in this chapter have already been published in:

Ulmke, P.A.\#, Sakib, M.S.\#, Ditte, P.\#, Sokpor, G.\#, Kerimoglu, C., Pham, L., Xie, Y., Mao, X., Rosenbusch, J., Teichmann, U., Nguyen, H.P, Fischer, A., Eichele G., Staiger, J.F., Tuoc T. (2021). Molecular Profiling Reveals Involvement of ESCO2 in Intermediate Progenitor Cell Maintenance in the Developing Mouse Cortex. Stem cell reports, 16(4), 968-984.

\# Authors with equal contribution.

Personal contributions: I was involved in analyses.

\subsection{Summary}

Intermediate progenitor cells (IPCs) are neocortical neuronal precursors. Although IPCs play crucial roles in corticogenesis, their molecular features remain largely unknown. In this study, we aimed to characterize the molecular profile of IPCs. We isolated TBR2 positive (+) IPCs and TBR2 negative (-) cell populations in the developing mouse cortex. Comparative genomewide gene expression analysis of TBR2+ IPCs versus TBR2- cells revealed difference in key factors involved in chromatid segregation, cell-cycle regulation, transcriptional regulation, and cell signaling. Notably, mutation of many IPC genes in human led to intellectual disability and caused a wide range of cortical malformations, including microcephaly, and agenesis of corpus callosum. Loss-of-function experiments in cortex-specific mutants of ESCO2, one of the novel IPC genes, demonstrate its critical role in IPC maintenance, and substantiate the identification of a central genetic determinants of IPC biogenesis. Our data provide novel molecular characteristics of IPCs in the developing mouse cortex.

\subsection{Introduction}

In developing cerebral cortex, IPCs are transit amplifying cells that express the T-box transcription factor TBR2 (Hevner, 2019). IPCs are basal derivatives of the multipotent radial glial progenitor cells (RGCs) in developing cortex, and they exclusively differentiate into glutamatergic neurons. Although IPCs are known to give rise to the majority of cortical neurons (Haubensak et al., 2004; Kowalczyk et al., 2009; Miyata et al., 2004; Noctor et al., 2004), the molecular factors which drive or maintain the transient proliferative capacity and neurogenic properties of TBR2+ IPCs in the SVZ niche remain not fully explored. The identification of the gene expression program that governs the genesis and maintenance of IPCs would improve our understanding of cortical development, and provide possible protocols to culture IPCs in vitro or generate these cortical progenitors by cell reprograming from other cell sources. Moreover, a description of the molecular features of IPCs can provide insights into the genes implicated in the etiology of pertinent neurological disturbances caused by defective IPC genesis.

To understand the molecular signatures of cell types in developing cortex, scRNA-seq analyses have been employed to provide the molecular identity of cell subtypes, including IPCs in mouse (Kawaguchi et al., 2008; Li et al., 2020b; Loo et al., 2019; Telley et al., 2016) and 
human cortex (Fan et al., 2018; Li et al., 2018a; Nowakowski et al., 2017; Pollen et al., 2015; Zhong et al., 2018). However, due to the threshold of high-throughput scRNA-seq, profiling cell type-specific gene expression is challenging. Comparisons between transcriptome analyses from purified cell populations have contributed additionally insightful molecular information about cortical cell subtypes (Albert et al., 2017; Amamoto et al., 2020; Arlotta et al., 2005; Molyneaux et al., 2015; Pinto et al., 2008).

In the present study, we used an antibody to label intranuclear TBR2 in single-nuclei suspensions isolated from E16.5 mouse cortex, and then sorted the TBR2+ cells (taken as IPCs) from the TBR2- cells (taken as non-IPCs). We then identified the expression of IPC-enriched genes by RNA-seq. Using high-throughput in situ hybridization (ISH) (Visel et al., 2004), we confirmed so far the SVZ-restricted expression of 392 novel IPC genes. The in situ expression of these genes are freely available online in an interactive database (https://gp3.mpg.de). A comparison of mouse IPC transcriptome and human phenotype annotations suggests that these IPC-enriched genes play important roles in cortical development in human as such patients with mutation variants display a wide range of cortical malformation and intellectual disability. Comparative genome-wide gene expression analysis of TBR2+ IPCs versus TBR2- cells revealed changes in key factors for chromatid segregation, cell-cycle regulation, transcription regulation and chromatin remodeling. Among them, establishment of sister chromatid cohesion $\mathrm{N}$-acetyltransferase 2 (ESCO2) was selected for confirmative studies. The cortex-specific mutagenesis for ESCO2 caused a massive depletion of the IPC population, thus validating that we identified a central genetic determinant of IPC maintenance.

\subsection{Results}

\subsubsection{Sorting and transcriptome profiling of intermediate progenitor cells in developing mouse cortex}

To compare the transcriptome profile of TBR2+ IPCs and TBR2- cells in mouse developing cortex (Fig. 2.1A), we isolated nuclei and established intranuclear immunofluorescent staining and FACS protocol (Fig. 2.1B). We used intranuclear TBR2 antibody labeling in single-nuclei suspensions isolated from E16.5 mouse cortices followed by cell sorting (Fig. 2.1C) (Sakib et al., 2021). Sorting gates were adjusted to purify TBR2+ IPC and TBR2- cell nuclei. Unlike unsorted nuclei suspensions, sorted nuclei suspensions were highly enriched (i.e., >99\%) in the desired cell type (Fig. 2.1C/D).

To understand the gene-regulatory difference in these cell populations, we generated RNA-seq libraries with three biological replicates for each cell populations. As anticipated, a high expression of canonical IPC genes was observed in the IPC population consistent with known in vivo expression patterns (Fig. 2.1E-F, Fig. S2.1A-C, Table S1). Comparing the expression of housekeeping genes, which locate on chromosome X (Xist, Pgk1, Hprt, Eif2s3x) and chromosome Y (Ddx3y, Eif2s3y) revealed their comparable expression level in samples from TBR2+ IPCs and TBR2-cells (Fig. S2.1C). The data suggests that TBR2+ and TBR2- cell populations were derived from a similar number of female and male embryos.

Next, we sought to identify genes with significant differences in expression level between the two cell populations and used these expression estimates to identify clusters. 
Cuffdiff 2 was used to identify 1119 enriched IPC genes (1050 protein-coding and 69 lncRNAs) and 1714 enriched non-IPC genes with significant differential expression between the cell types (p-value $<0.01 \& \mid$ fold change $\mid>1.0$, Fig. 2.1E/F). The IPC-enriched genes were annotated into different functional categories (Fig. 2.1G, Fig. S2.1D-E, Table S2, see Experimental Methods). The significance of such predominating gene clusters was analyzed and is discussed later.

The reliability of our analyses was ensured by validating the expression pattern of the identified IPC-enriched genes in mouse developing cortex. In situ hybridization (ISH) for 392 of such genes confirmed their restricted expression in SVZ in the E14.5 cortex, which is consistent with their RNA expression profiles (Table S3). To further validate the quality of our RNA-seq data, we performed immunohistochemical analysis of the E15.5 mouse cortex for ESCO2 as one of novel IPC factors. ESCO2 protein expression was mainly observed in the germinative zone of the developing cortex, especially the basal aspect (Fig. 2.1H). Quantification revealed that most of the cells expressing ESCO2 also expressed the IPC marker TBR2, but to a less extent in cells in the transition stage between RGC-IPC (PAX6+/TBR2+) and in RGCs (PAX6+) (Fig. 2.1I).

Previous studies, which have characterized the transcription profiles of single cells isolated from the developing mouse cortex, have generated a repository of genes enriched in each of the murine cortical cell types (Kawaguchi et al., 2008; Li et al., 2020b; Loo et al., 2019; Telley et al., 2016). To identify novel IPC genes that might play essential roles in the development of this cell type, we compared the list of mouse IPC genes from these scRNA-seq experiments and from sorted TBR2+ IPCs (this study) and found that our list of IPC-enriched genes contains most of the previously identified IPC genes (Fig. S2.1E). Intriguingly, 961 out of the 1121 IPC enriched genes from this study (Fig. S2.1D) were not present in any of the gene lists of the above mentioned studies. GO analysis indicated that these novel IPCs encode for proteins belonging to different families of factors, such as chromatin/epigenetic factors, DNA, RNA-binding factors, and post-transcription/translation modification factors (Table S2, Fig. S2.1E).

Together, the results demonstrate an efficient isolation of mouse TBR2+ IPCs, which allow adequate molecular profiling. 


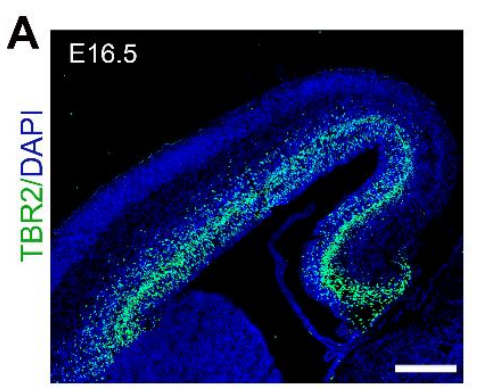

B
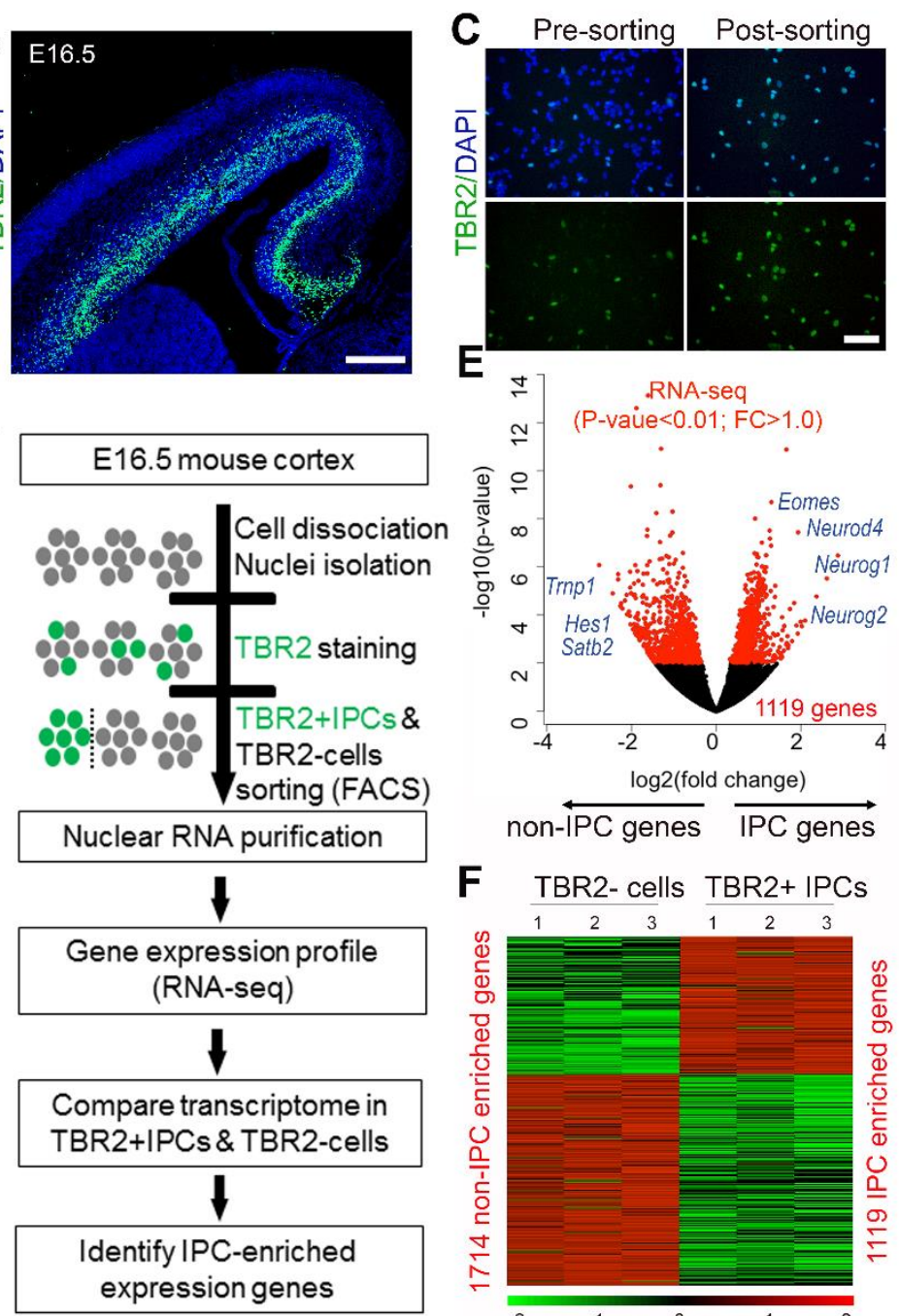
non-IPC genes $\stackrel{\text { IPC genes }}{\longleftrightarrow}$
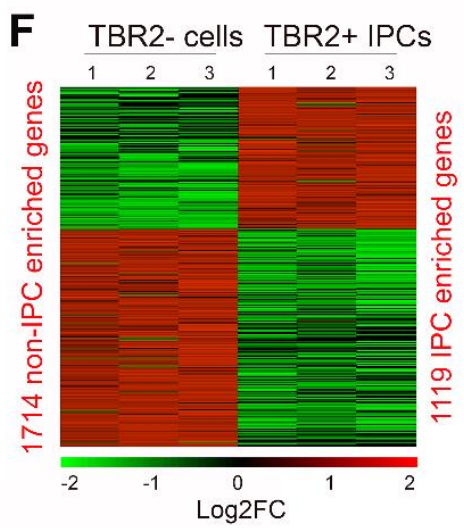

D

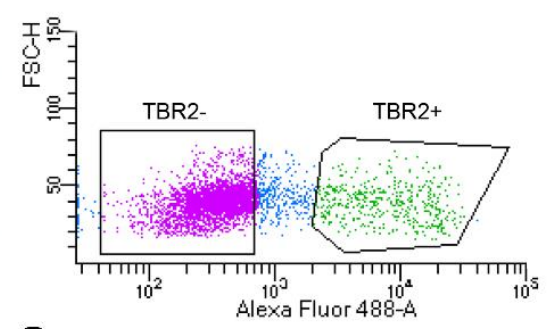

G

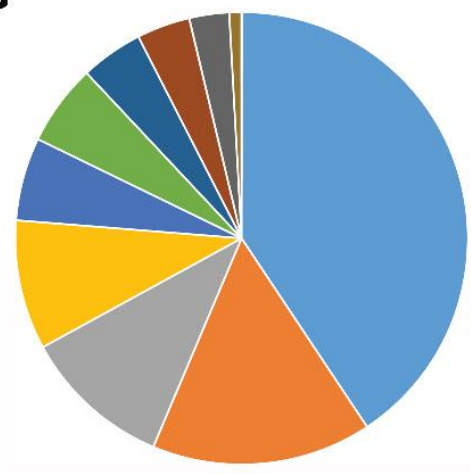

Top 10 of Functional Annotation Clusterings (Enrichment Score)

Cell cycle (36.65)

Chromosome segregation (14.16)

Cell signaling (9.59)

- Transcription regulation (8.35)

DNA damage/repair (5.35)

Microtubule/kinesin (5.24)

Nervous system development (4.02)

DNA replication (3.43)

Extracellular matrix (2.57)

Chromatin organization $(0.78)$
H

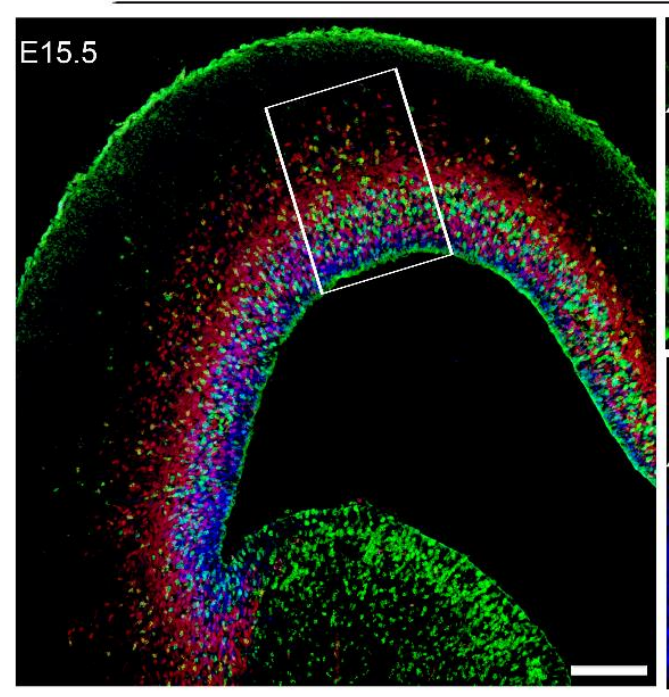

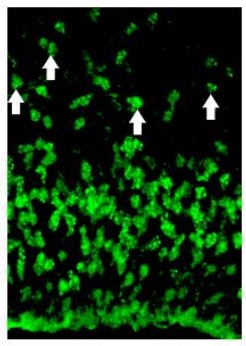

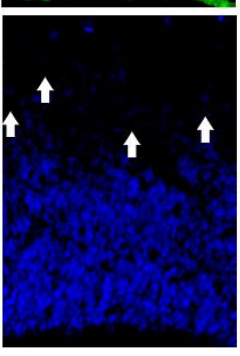

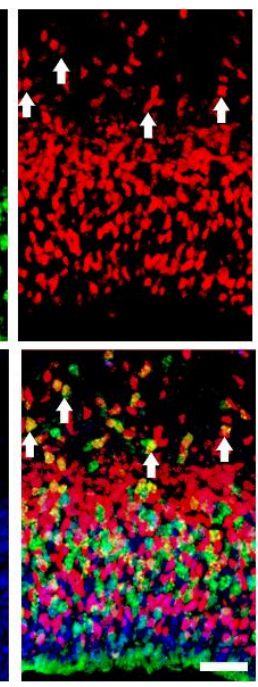

I ESCO2+/PAX6-/TBR2- ESCO2+/PAX6-/TBR2+ - ESCO2+/PAX6+/TBR2+ - ESCO2+/PAX6+/TBR2-

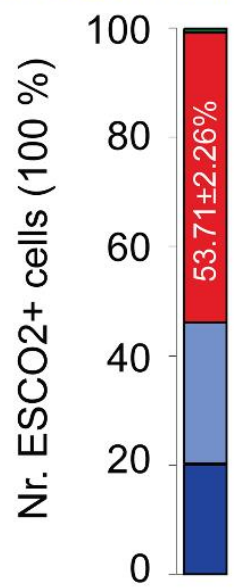

Figure 2.1 Cell Sorting and Gene Expression Profiling of Mouse TBR2+ IPCs

(A) Micrograph showing the E16.5 mouse cortex immunostained with TBR2 antibody. Counterstaining was done with 4',6-diamidino-2-phenylindole (DAPI). (B) An illustration of the experimental design used to sort out TBR2labled IPCs and subsequent nuclear analysis to compare the transcriptional profile of IPCs in mouse. (C) Representative images of pre- and post-sorted cell suspensions from mouse cortex stained with TBR2 antibody. 
Counterstaining was done with DAPI. (D) Representative plot showing sorting gates for TBR2+ and TBR2- cells from mouse and human cortex. (E and F) Volcano plot (E) and heatmap (F) showing the enrichment of IPC and non-IPC genes in corresponding sorted cell populations. (G) Pie chart showing proportions of the enrichment score of the top ten functional annotation clustering in IPCs. (H) Micrograph of immunohistochemical (IHC) staining showing the E15.5 wild-type mouse cortex at low and high magnification stained with ESCO2, TBR2, and PAX6 antibodies. Cortical area with high magnification is indicated by a white box. Arrows point to TBR2+ IPCs coexpressing ESCO2 but not PAX6. (I) Composite bar graph showing the quantitative proportion of ESCO2+ cells co-expressing PAX6 or TBR2 or both in germinal zone of the E15.5 mouse cortex. $n=6$ experimental replicates. Scale bars, $100 \mu \mathrm{m}(\mathrm{A})$ and $50 \mu \mathrm{m}(\mathrm{C}$ and $\mathrm{H})$.

\subsubsection{Predominance of mitotic cell cycle and mitotic chromatid segregation-related gene signatures in IPCs}

Previous studies revealed that IPCs are transient cortical progenitors, which actively undergo mitotic cell divisions (Hevner, 2019). Consistent with these features of IPCs, many of the genes in the top GO pathways belong to cell cycle and cell division-related categories (Fig. 2.2A). Remarkably, our gene expression profiling revealed genes encoding for many cyclin and cyclin cofactors are highly expressed in IPCs (Fig. 2.2B/C, Table S4). Examination of these cell cycle regulation genes in subtypes of IPCs might offer a yardstick for distinguishing neurogenic IPCs from proliferative IPCs.

During cell division, chromosomes need to be segregated and evenly distributed among daughter cells to ensure accurate passing of genetic information to the next generation. In addition to the alterations in mRNA levels for genes involved in cell cycle regulation, high expression of genes related to DNA replication, repair, and chromatid segregation were observed (Fig. 2.2A, Table S4). Particularly, expression of genes encoding for subunits of the chromosome segregation machinery is highly enriched in IPCs, e.g. Cohesin complex (Sgoll, Sgol2, Smc3, Rec8, Cdca5, and Wapal), Condensin complex (Ncapd2, Ncapd3, Ncapg, Ncaph, $\mathrm{Smc2}$, and Smc4), Minichromosome maintenance complex (Mcm2, Mcm3, Mcm4, Mcm5, Mcm6, Mcm7, and Mms22l), and SMC5-SMC6 complex (Smc5, Smc6, Nsmce2, and Nsmce4a) (Fig.2.2D/E, Table S4). Single gene factors (e.g. Esco2, Spag5, Ncapg) involved in chromosome segregation were also identified in IPCs (Fig. 2.2D/E).

The results shown here indicate that the expression of many cell cycle and chromatid segregation genes is associated with, and supportive for, the highly active cell division of IPCs. 


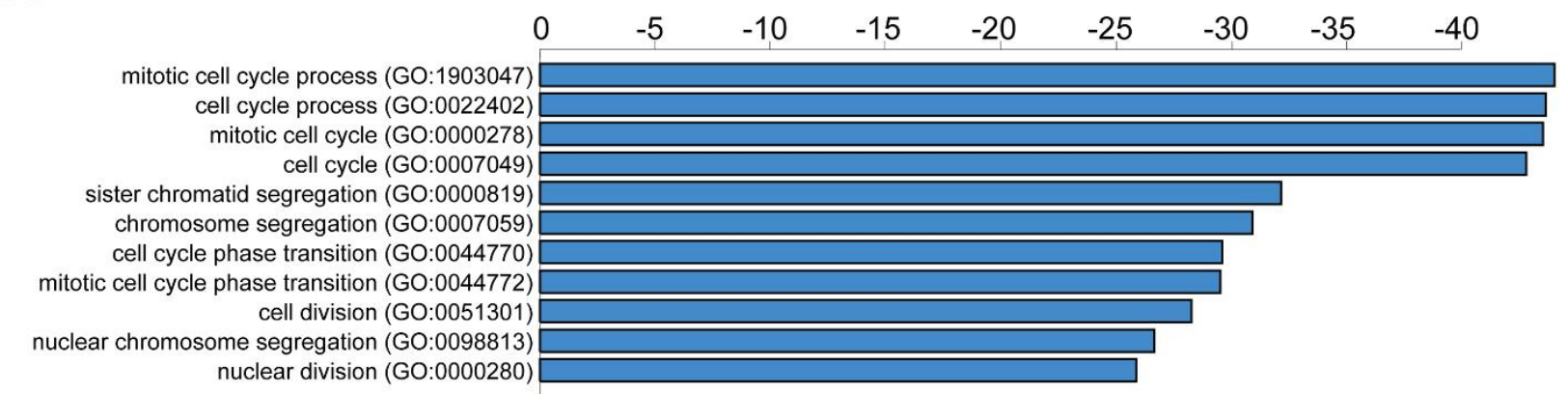

\section{B Cell cycle-linked genes} Total number of genes (392) Genes with validated SVZ expression (64): Ange/2, Atrx, Aurka, Aurkb, Brca1, Bub1, Bub1b, Bub3, Cab391, Ccna2, Ccnb1, Cdc25c, Cdc5l, Cdca2, Cdca5, Cdk1, Cdk2, Cdk4, Cdk5rap3, Cdkn1b, Cdkn2c, Cenpj, Chek2, Clspn, Cnot8, Crlf3, Ctnnb1, Cul1, Dgkz, E2f1, E2f8, Espl1, Fbxo5, Fgfr3, Foxm1, Gas2, Gen1, Gmnn, Gtse1, Hbp1, Kntc1, Mad2l1, Mad2I2, Mki67, Mtbp, Nae1, Pcid2, Pidd1, Pml, Ptprk, Rpa2, Sox4, Ska1, Spag5, Spc25, Strada, Terf1, Tfdp2, Tipin, Top2a, Trp53, Trp53inp1, Ttk, Zfp655

\section{Chromosome segregation} -linked genes

\section{Total number of genes (121)}

Genes with validated SVZ expression (84): Akap8I, Anapc4, Atrx, Aurkb, B9d2, Becn1, Birc5, Brca1, Bub1, Bub1b, Bub3, Conb1, Cdc27, Cdca2, Cdca5, Cdca8, Cenpa, Cenph, Cenpn, Cenpo, Cenpp, Cenpu, Cep55, Cep63, Chmp1b, Chtf18, Ctnnb1, Dscc1, Dsn1, Ect2, Ercc6l, Esco2, Espl1, Fam96a, Fancd2, Gen1, Hdac8, Hjurp, Incenp, Kif11, Kif22, Kif23, Kif2c, Kif4, Kntc1, Mad2/1, Mad2/2, Mtbp, Ncapd2, Ncapd3, Ncapg, Ncaph, Ndc80, Nde1, Nsmce2, Nuf2, Nup37, Nusap1, Pcid2, Pibf1, Pmf1, Prc1, Psrc1, Pum1, Racgap1, Rad18, Rcc1, Rec8, Sgol2, Ska1, Ska2, Ska3, Smarcad1, Smc2, Smc4, Smc5, Smc6, Spag5, Spc24, Spc25, Terf1, Top2a, Ttk, Vps $4 b$

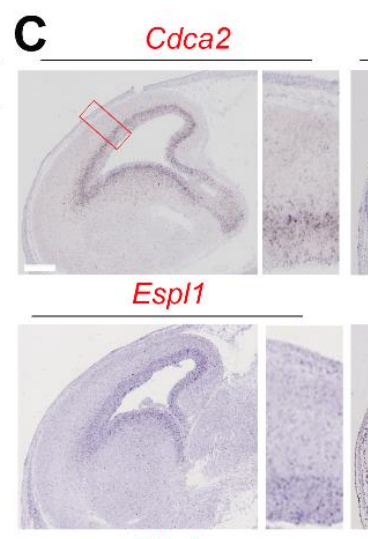

Ska1
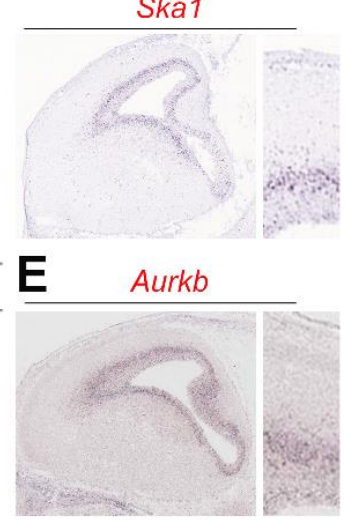

Dscc1

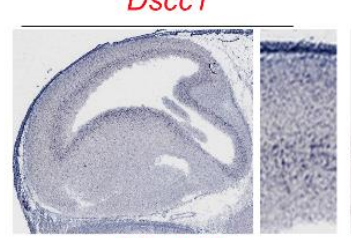

Ncapd3

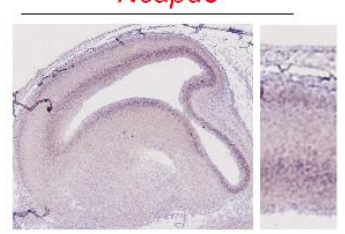

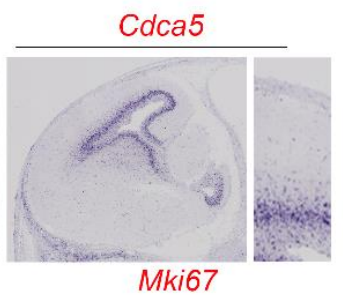

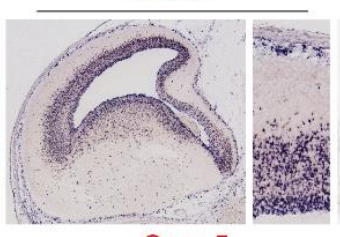

Spag5

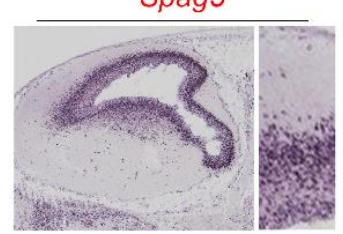

Cenph

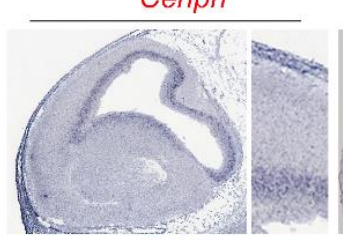

Kif11

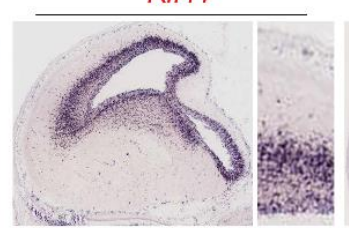

Sgol1/Shugoshin

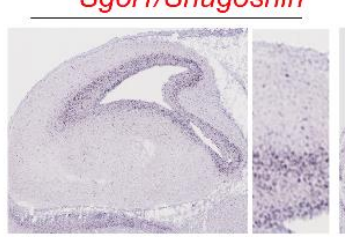

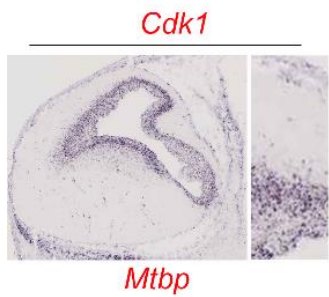

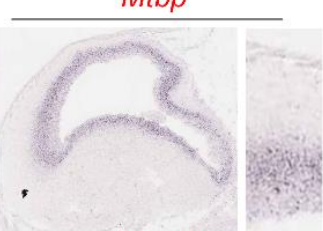

Spc25

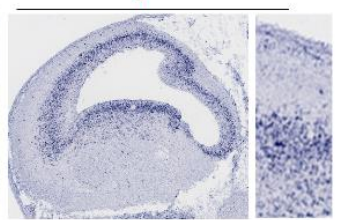

Chtf18

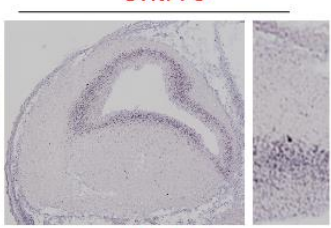

Ncapg

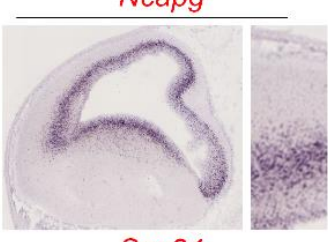

Spc24

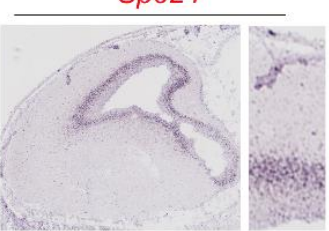

Figure 2.2 Expression of Many Cell-Cycle and Chromatid-Segregation Genes Are Enriched in IPCs

(A) Graphical representation of gene ontology analysis with terms related to cell cycle and segregation of chromatids. (B and D) List of the genes identified in IPCs that functionally fall under cell-cycle- and chromosomesegregation-related processes, respectively. ( $\mathrm{C}$ and $\mathrm{E}$ ) Respective array of micrographs showing in situ hybridization of examples of the identified genes (highlighted red in B and D) with distinctive expression in the developing mouse cortical subventricular zone, and related to cell-cycle and chromosome-segregation events. Magnified cortical region is shown by a red box in (C). Scale bar, $100 \mu \mathrm{m}$. 


\subsubsection{Many IPC-enriched genes belong to signaling pathways}

Pathway enrichment analysis revealed that several brain-regulating signaling pathways are significantly enriched in IPC genes, including the P53-Caspase cascade, Hippo, Notch, FoxO, PI3K-Akt, Axon guidance, and Fanconi anemia signaling pathway (Fig. 2.3A). Corroborating the results from the transcriptomics analysis, we confirmed an enrichment of several genes belonging to these signaling pathways in SVZ (Fig. 2.3B-Q). The identified signaling pathways may play unique roles in the proliferation, differentiation and/or survival of IPCs during cortical development.

Cell lineage tracing experiments with TBR2-CreER indicated that the majority of IPC derived clones $(\sim 66 \%)$ generate one daughter cell as neuron and another as apoptotic cell, indicating asymmetric cell death (Mihalas and Hevner, 2018). The observed remarkable abundance of apoptosis of IPC daughter cells is in accordance with previous findings of high level of cell death in the SVZ (Blaschke et al., 1996; Thomaidou et al., 1997). These outcomes are congruent with our GO analysis, which revealed that many genes belonging to the P53 signaling cascade and caspase signaling pathway, which lead to apoptosis, are enriched in IPCs (Fig. 2.3B-E, Table S5). Indeed, immunohistochemical analysis indicated a large proportion of CASP3+ cells immunoreactive with TBR2 in the developing mouse cortex at E16.5 (Fig. S2.2), corroborating the finding that more than half the progenies of IPCs undergo apoptotic cell death in the normally developing cortex (Mihalas and Hevner, 2018).

Hippo signaling is necessary for cell fate and organ size determination (Zheng and Pan, 2019). Emerging evidence shows the involvement of the Hippo signaling alone or cooperatively with other signaling pathways in brain development (Ouyang et al., 2020). As shown in our transcriptomic analysis, genes involved in the Hippo signaling pathway are prominently expressed in IPCs (Fig. 2.3F/G) and likely critical for the regulation of cortical size via control of IPC genesis (Kostic et al., 2019). Other signaling pathways such as the Delta-Notch (Fig. 2.3H/I), FoxO (Fig. 2.3J/K), PI3K-Akt (Fig. 2.3L/M), Axon guidance (Fig. 2.3N/O), and Fanconi anemia (Fig. 2.3P/Q) were also identified to be enriched in IPCs. These signaling pathways may play critical roles in the normal progression of brain morphogenesis via modulation of IPC biogenesis.

Together, the enrichment of signaling pathway genes in the sorted IPCs is indicative of their necessity in sustaining the neuronal progenitor properties of IPCs in the SVZ niche and to permit their function in cortical morphogenesis. 
A

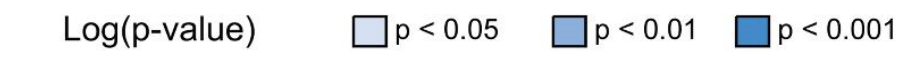

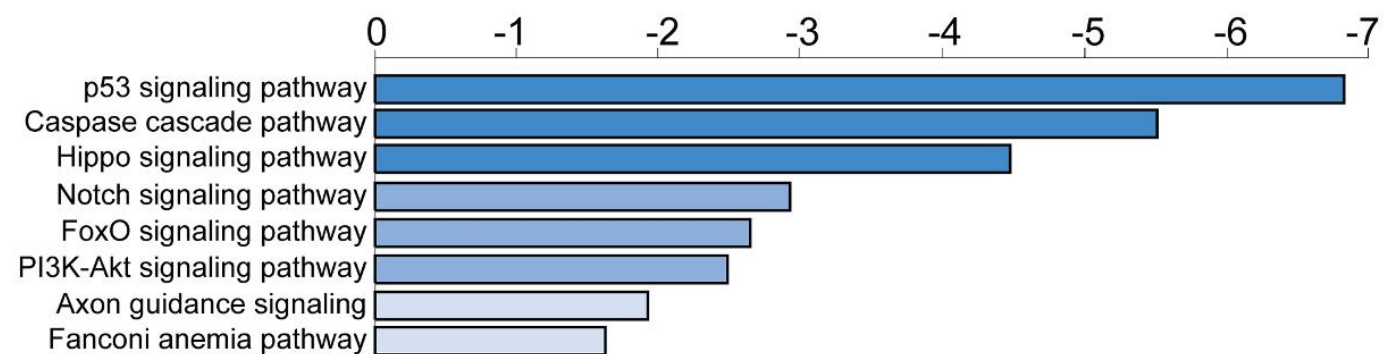

B p53 signaling-linked genes Total number of genes (16): Ccnb1, Conb2, Cond2, Cone1, Cone2, Cdk2, Cdk6, Chek2, Gadd45a, Gadd45g, Gtse1, Perp, Pidd1, Rrm2, Sesn1, Sesn2

D Caspase cascade-linked genes Total number of genes (14): Casp2, Casp6, Casp8ap2, Cfl2, Cradd, Diablo, Gas2, Numa1, Pidd1, Ripk1, Srebf1, Top1, Traf2, Trp53

F Hippo signaling-linked genes Total number of genes (20): Amot, Birc5, Cond2, Ctnna1, Fzd2, Fzd3, Fzd6, Gli2, Lef1, Limd1, Mpp5, Stk3, Tcf7/2, Tead1, Tead2, Tead3, Tgfb2, Tgfbr2, Wnt10a, Wnt5a

H Notch signaling-linked genes Total number of genes (9): DII1, DII3, DII4, Hdac1, Mam/3, Mfng, Ncstn, Notch1, Numb

J FoxO signaling-linked genes Total number of genes (15): Bcl2111, Ccnb1, Ccnb2, Ccnd2, Cdk2, Foxo3, Gadd45a, Gadd45g, Irs1, Pck2, Pik3r3, Slc2a4, Stat3, Tgfb2, Tgfbr2

L PI3K-Akt signaling genes Total number of genes (28): Bc/2l11, Brca1, Cond2, Cone1, Cone2, Cdk2, Cdk6, Col4a5, Col5a1, Col5a2, Creb3/2, Creb5, Ddit4, F2r, Foxo3, Irs1, Itga4, Itga7, Itga9, Itgb1, Lama2, Lama5, Lamb2, Lpar1, Lpar4, Lpar6, Pck2, Pik3r3

N Axon guidance-linked genes Total number of genes (13): Cxc/12, Dcc, Epha3, Epha4, Ephb4, Itgb1, Nfatc4, Ntng2, Pak4, Sema3c, Sema3g, Sema5a, Unc5d

P Fanconi anemia-linked genes Total number of genes (7): Brca1, Brip1, Eme1, Fancd2, Fanci, Rad51, Ube2t
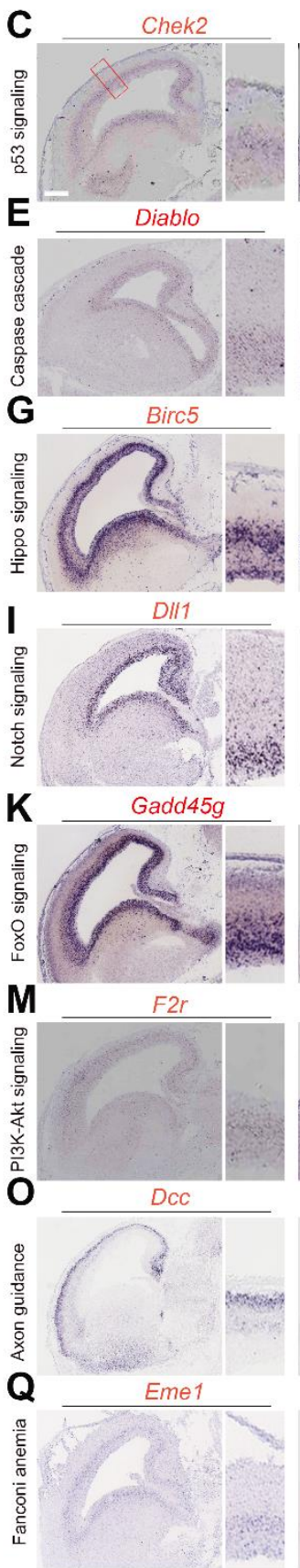
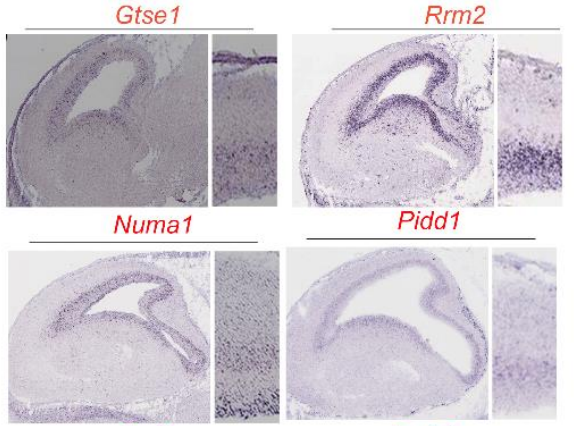

Tgfb2

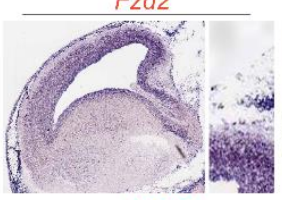

Hdac1

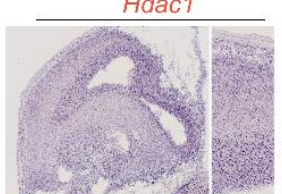

Stat3

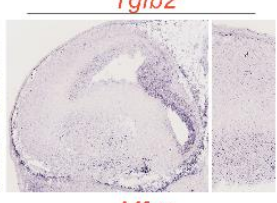

Mfing

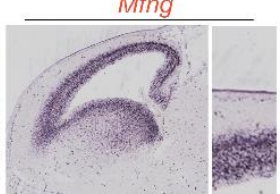

S/c2a4

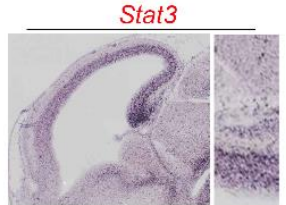

Pck2
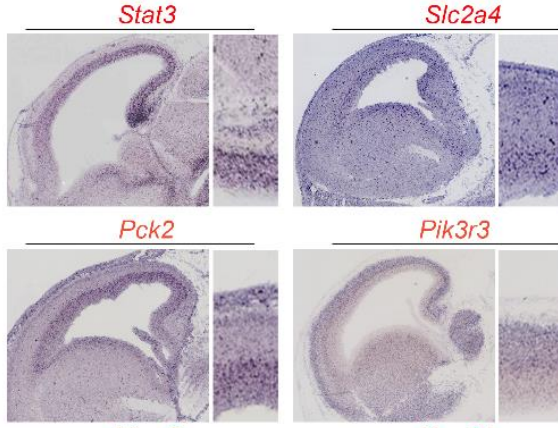

Ntng2

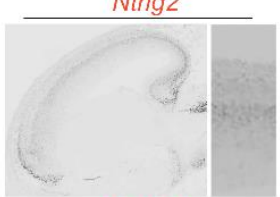

Fancd2

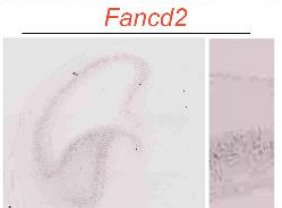

Pik3r3

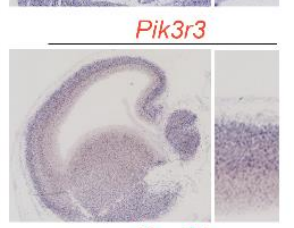

Unc5d
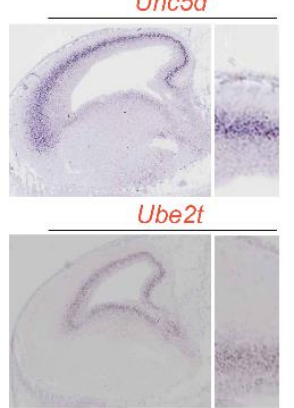

Figure 2.3 IPC-Enriched Genes Encode for Variety of Signaling Pathway Factors

(A) Graphical representation of the top-ranked signaling pathways that are prominent in IPCs. (B, D, F, H, J, L, $\mathrm{N}$, and P) List of the genes identified in IPCs that are involved in the P53, Caspase, Hippo, Notch, FoxO, PI3KAkt, Axon guidance, and Fanconi anemia signaling pathways, respectively. (C, E, G, I, K, M, O, and Q) Respective array of micrographs showing in situ hybridization of examples of the identified signaling pathway-linked genes (highlighted red in the adjoining gene list) with distinctive expression in the developing mouse cortical subventricular zone. Magnified cortical region is shown by a red box in (C). Scale bar, $100 \mu \mathrm{m}$. 


\subsubsection{Identification of new IPC-specific transcription and epigenetic regulators}

Many of the genes identified in IPCs were found to participate in the regulation of transcription, chromatin remodeling, and other epigenetic processes (Fig. 2.4A). We first looked for epigenetic and chromatin regulators, which are highly expressed in IPCs. We found enrichment of epigenetic genes in the sorted IPCs: a total of 66 genes, 25 of which were validated by ISH (Fig. 2.4B/C, Table S6). Such epigenetic genes include Deacetylase genes, genes related to the PRC2 complex, and genes encoding for Methyltransferase domain-containing proteins.

A set of IPC genes which encode for protein factors that form complexes to regulate chromatin dynamics was identified. In all, we found 52 such genes markedly expressed in IPCs, and with distinctive expression in the SVZ, at least for 24 of them (Fig. 2.4D/E, Table S6). The identified chromatin modification related-genes belong to the following classes of chromatin remodelers: SWI/SNF superfamily-type complex, and ISWI-type complex, NuRD/CHD complex (Table S6).

Besides epigenetic and chromatin regulators, our data provided a context to examine the relative contribution of specific non-coding (nc)RNAs, and TFs, to IPC identity and/or regulation. We identified 69 known long non-coding (lnc)RNAs and 3 small nucleolar RNAs (sno) RNA genes, with significantly higher level of expression in TBR2+ IPCs compared with TBR2- cells $(\log 2 \mathrm{FC}>1.0$, p-value<0.01) (Fig. 2.4F/G, Table S6). Among these ncRNAs, ISH analysis confirmed the restricted expression of three lncRNAs (A930024E05Rik, 5330426P16Rik, 9630028B13Rik, Fig. 2.4G) in SVZ of the developing cortex.

We identified 104 IPC genes encoding TFs belonging to more than four protein families (Fig. 2.4H/I, Fig. S2.3, Table S6). C2H2 type zinc finger protein family was the most enriched protein family, with 47 up-regulated genes, followed by basic-helix-loop-helix (bHLH)/Myb, and homeobox protein families with 9 up-regulated genes each. The LIM TF family genes were also found in the purified IPCs (Fig. S2.3A-I). The genes encoding for TFs, which were found to exhibit a high expression in IPCs included many known key regulators of neurogenesis such as Eomes (Tbr2), Ngn1, Ngn2, NeuroD1, and Bag2, as well as many yet uncharacterized genes (e.g., Nhlh1, Csrpl, and Mybl2; Fig. 2.4H) that may prove to be novel regulators of cortical development.

Next we determined which of the transcription factors interact physically or functionally using the STRING database. This revealed a highly interconnected network formed by IPCenriched TFs. Several TFs formed a network hub. Among them Cbfa2t2, Neurog2, Neurog1, Stat3, Neurod1, and Tcf3 appear to be in the center of the network as they interact with many other TFs (Fig. 2.4J). This raises the possibility that the components of this TF network are key determinants in IPC biogenesis.

Taken together, our findings indicate major elements of the transcriptional and epigenetic machinery distinctively present in mouse IPCs. 


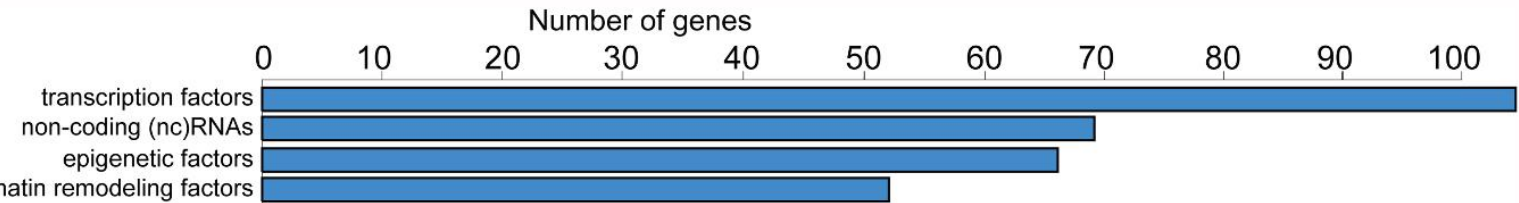

B Epigenetic factor genes

Total number of genes (66)

Genes with validated SVZ expression (25): App/2,Atf7ip, Atrx,Btg1,Btg2, Ciapin1, Eed,Ezh2, Gcsh,H2afy2,Hdac1,Hdac3, Hd ac8,Hmg20b, Hsd17b10, Jarid2, Mettl14,Mt $r$, Ncor2,Prdm16, Rbbp7, Sall1, Snrpb, Trmt $2 b$, Tyms

\section{Chromatin factor genes}

Total number of genes (52)

Genes with validated SVZ expression (24): Actl6a,Asf1b,Brca1,Brip1,Brd8, Cebpg,Cenpa,Cenph,Cenpn,Cenpo,Cenp p,Cenpq,Cenpu,Chrac1,Ctnnb1,Hjurp,Mis 18bp1,Ms/3,Mta2, Nasp,Phf21a,Psme4,S marcad1,Smarcd2,Spty2d1

\section{$F \quad$ IncRNA genes}

Total number of genes (69)

Genes with validated SVZ expression (3): A930024E05Rik,5330426P16Rik,9630028B1 3Rik

\section{H Transcription factor genes}

Total number of genes (104)

Genes with validated SVZ expression (47):Actl6a,Akna, Cebpg, Csrp1,E2f8,Ebf2, Eomes, Fubp3,H2afy2, Hes6, Hey1, Hmgb2 Jarid2,Kcnip3,Kif2c,Mbd3,Meis2,Mybl1, Myb/2,Myt1,Neurod1, Neurod4,Neurog1,N eurog2,Nfkb2,Nfyc,Nhlh1,Nono,Nrf1, Orc2 Otx1, Phf21a, Phf21b, Pou2f1, Rbpj, Smarc d2, Sox4,Sp5, Srebf1, Stat3, Tcf12, Tcf3, Tfa p2c, Thrap3,Trp53,Uhrf1,Zfp219,Zkscan3
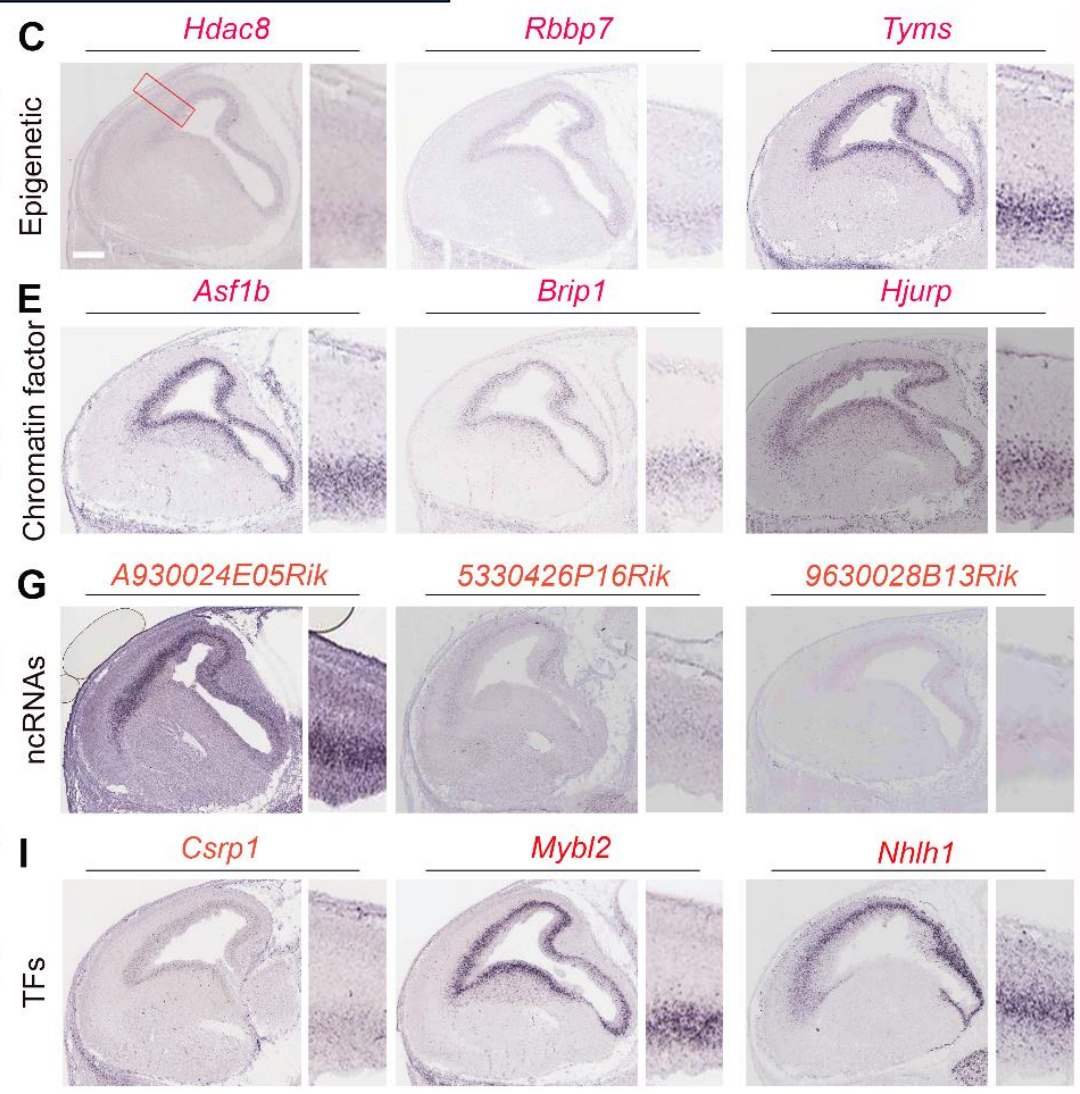

$\mathbf{J}$

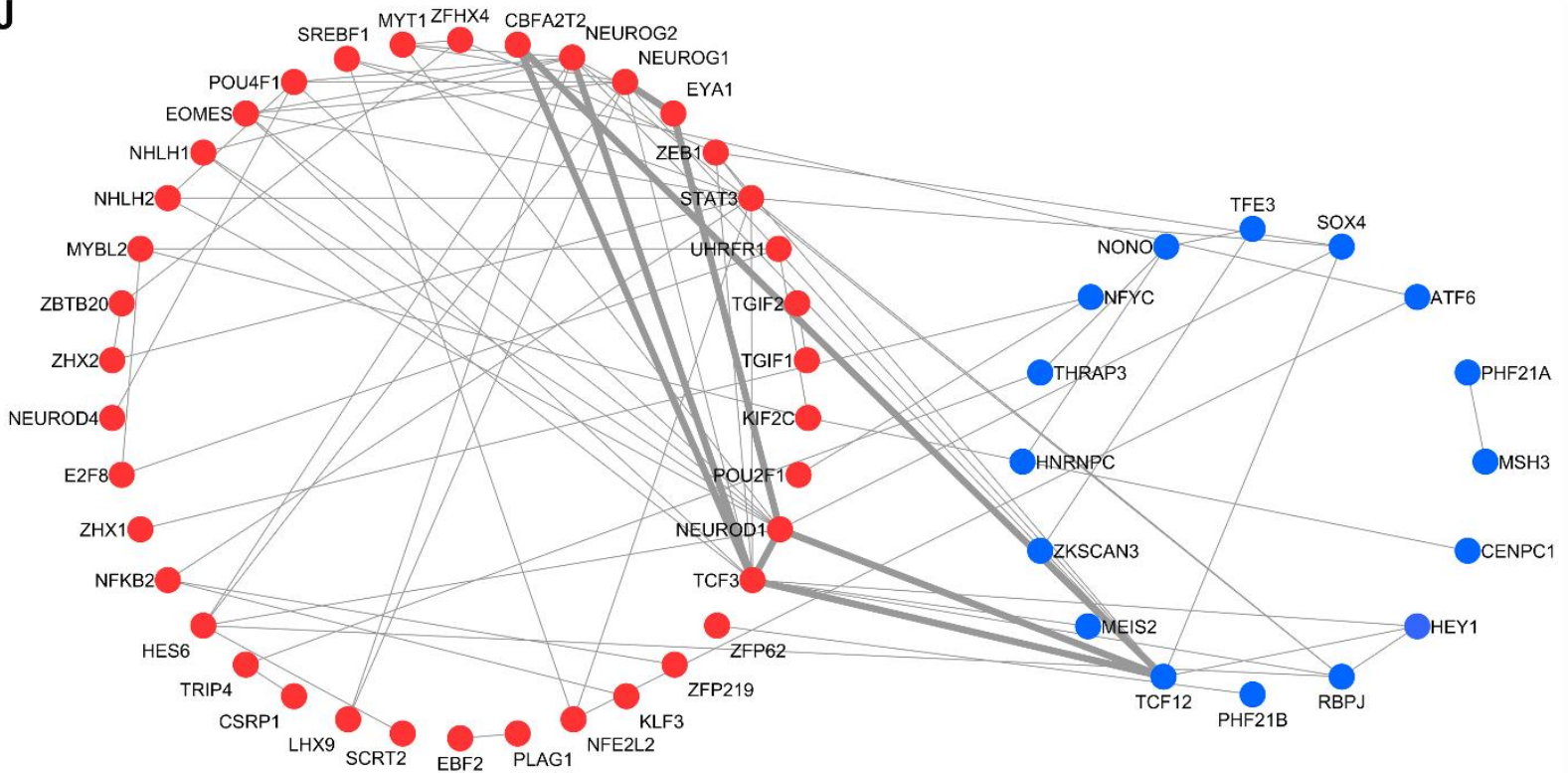

Figure 2.4 Identification of Novel IPC-Specific Transcription Regulators

(A) Graphical representation of the total number of newly identified genes and their categorization in IPCs that have the potential to regulate transcription. (B, D, F, and H) List of the genes identified in IPCs that are transcription regulators and can be grouped as epigenetic, chromatin, lncRNA, and transcription factors, respectively. (C, E, G, and I) Respective array of micrographs showing in situ hybridization of examples of the identified transcription regulation genes (highlighted red in the adjoining gene list) with distinctive expression in the developing mouse cortical subventricular zone. Magnified cortical region is shown by a red box in (C). Scale 
bar, $100 \mu \mathrm{m}$. (J) Protein-protein interaction network of the IPC-enriched transcription factors (TFs). The list of TFs were imported into the STRING database (http://string-db.org/), and the physical or functional interactions between the differentially expressed transcription factors were extracted using the default settings. The red and blue nodes represent IPC-enriched TFs with $\log 2 \mathrm{FC}>1.0$ and $0.3<\log 2 \mathrm{FC}<1.0$, respectively. The thin lines indicate low interaction score $(<0.4)$ while the thick lines indicate medium or high interaction score $(\geq 0.4)$.

\subsubsection{Gene expression profiling suggests mutations of IPC-enriched genes have implications for cortical neurodevelopmental disorders in human}

Recent single-cell transcriptomic analysis of the human developing cortex identified a large set of IPC genes (Fan et al., 2018; Li et al., 2018a; Nowakowski et al., 2017; Pollen et al., 2015; Zhong et al., 2018) and IPC lncRNAs (Liu et al., 2016). To further study the developmental and evolutionary origin of the transcriptional signature of IPC cells, we compared these published scRNA data for human developing cortex to those for mouse developing cortex (Kawaguchi et al., 2008; Li et al., 2020b; Loo et al., 2019; Telley et al., 2016) and to bulk RNAseq for mouse TBR2+ IPCs (this study, Fig. S2.4). The comparisons revealed not only remarkable match between the two species, but also highlighted an expanded gene expression program in human IPCs (Fig. S2.4).

Mutations of the IPC-specific gene Tbr2/Eomes cause microcephaly and a wide range of cortical anomalies in both rodent (Arnold et al., 2008; Mihalas et al., 2016; Sessa et al., 2008) and human (Baala et al., 2007). In addition to congenital microcephaly, the affected individuals presented with dilatation of cerebral ventricles, agenesis of corpus callosum, polymicrogyria, and dysgenic cerebellum (Baala et al., 2007). The affected children also exhibited severe motor deficits, with hypotonia and intellectual disability (Baala et al., 2007).

To identify a potential involvement of these common IPC genes (Fig. S2.4B), which were found both in developing cortices from mouse (this study) and human (Fan et al., 2018; Li et al., 2018a; Nowakowski et al., 2017; Pollen et al., 2015; Zhong et al., 2018), in human diseases, we performed systematically Human Phenotype Ontology (HPO) analysis (Robinson et al., 2008) (Fig. 2.5A). Mutations of many IPC genes were found to be associated with intellectual-disability phenotype, thus supporting the idea that the perturbation of many biological pathways in IPCs can undermine cognitive development. In order to determine possible convergence of the various molecular pathways on intermediate phenotypes within the scope of intellectual disability, including brain structure malformations, we looked for correlated phenotypes among the 134 intellectual disability genes (Fig. 2.5B/C, Table. S7). We identified two major associated phenotypes: (1) microcephaly, and (2) corpus callosum agenesis (Fig. 2.5D-G, Table. S7). This observation supports the findings that IPCs generate most of the cortical projection neurons, especially, upper layer/callosal neurons, which are necessary for appropriate cortical size and proper establishment of cortical neuron connections across the corpus callosum.

In summary, many gene sets encode for components of the transcriptional, chromatin, and signaling machineries in mouse IPCs, with known or putative regulatory function in cell division, proliferation, differentiation and survival (Fig. 2.5H). Our data supports the possibility that major elements of the mouse IPC transcriptome may be conserved in human and play important roles in cortical development, with their mutations plausibly underlying cortical malformations and dysfunction in both species. 


\section{A Human Phenotype Ontology}

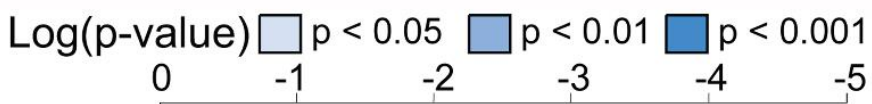

abnormality of the cerebrum (HP:0002060) abnormality of the forebrain morphology (HP:0100547) abnormality of skull size (HP:0000240) aplasia/hypoplasia of central nervous system (HP:0002977) aplasia/hypoplasia of the cerebrum (HP:0007364) abnormality of the forehead (HP:0000290) abnormality of chromosome segregation (HP:0002916) intellectual disability (HP:0001249) abnormal shape of the frontal region (HP:0011218) abnormality of the cerebral white matter (HP:0002500)

microcephaly (HP:0000252)

abnormality of the orbital region (HP:0000315) psychomotor developmental delay (HP:0001255) abnormality of the cerebral subcortex (HP:0010993) cerebral hypoplasia (HP:0006872) dysplastic corpus callosum (HP:0006989) agenesis of corpus callosum (HP:0001274) abnormal corpus callosum morphology (HP:0001273) aplasia/hypoplasia of the corpus callosum (HP:0007370)

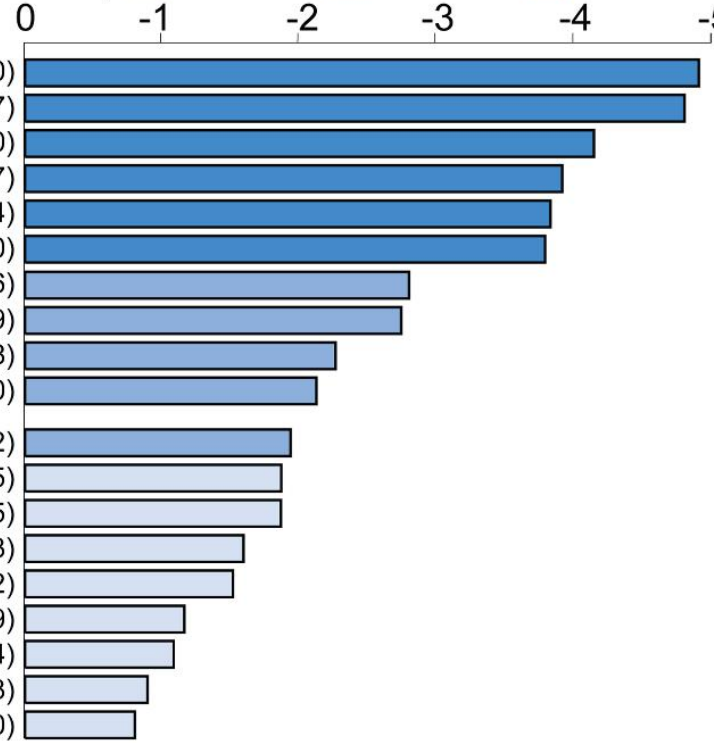
$-5$

B Intellectual disability-linked genes Total number of genes (134)

Genes with validated SVZ expression (48): ADSL, AGA, AIFM1, ALDH3A2, ALDH7A1, ATP7A, ATRX, BCAP31, BRIP1, CCDC88C, CDT1, CENPJ, CEP135, CEP63, CKAP2L, CRKL, CTNNB1, CXCL12,CWC27, DCC, DPYD, EED, ESCO2, EZH2, FGFR3 GTF2E2, HDAC8, HSD17B10, IER3IP1, IKBKG, KIF11, KIF4A, LARP7, MEIS2, NDE1, NONO, PHF21A, PHF6, PSAT1, RAD51, RBPJ SLC16A2 SLC2A1, SNAP29, SNRPB, ST3GAL5, STRADA, TACO1, TCF12, TINF2, VRK1, UBE2T, ZBTB18

D Microcephaly-linked genes

Total number of genes (77)

Genes with validated SVZ expression (33): ADSL, AGA, ATP7A, ATRX, BCAP31, BRCA1, BUB1B, CDT1, CENPJ, CEP135, CEP63, CKAP2L, CTNNB1, DPYD, ESCO2, HDAC8, IER3IP1, IKBKG, KIF11, LARP7, MCM4, MGME1, NDE1, NEUROG1, PEX19, PHF6, PSAT1, RAD51, SASS6, SLC16A2, ¿ SLC2A1, SNAP29, SNRPB, VRK1, ST3GAL5, ZBTB18

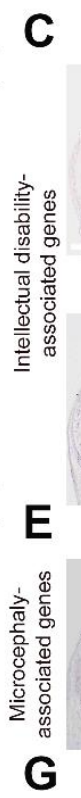

C

E

G

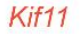

Kif11
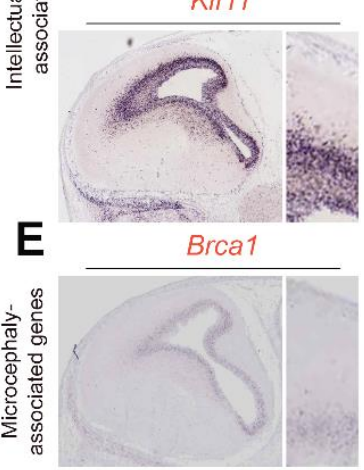
Dpyd

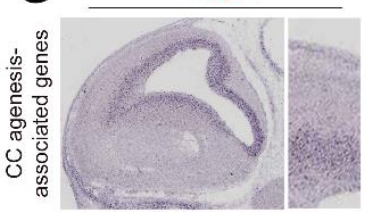

Cdt1

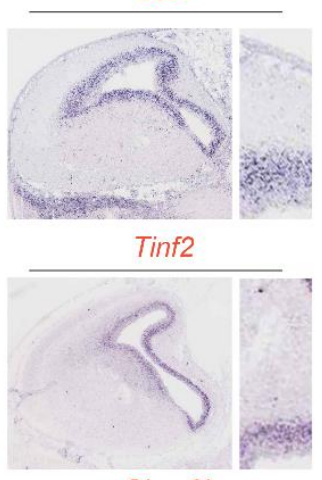

Ckap2l

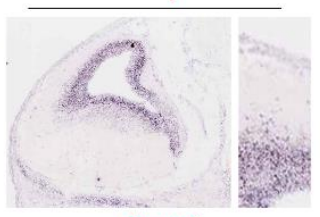

Sass6

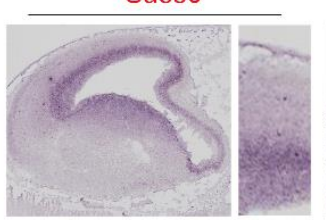

Cxcl12

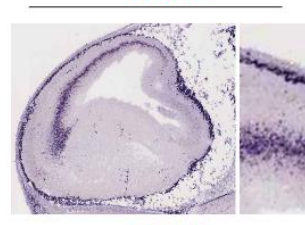

Ube $2 t$

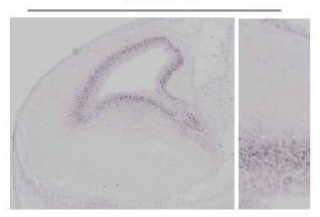

Rad51

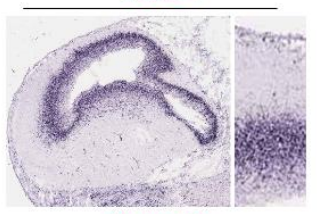

Sema3c

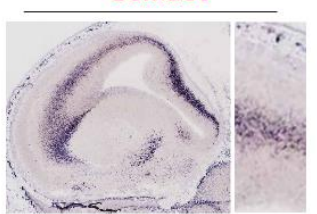

F Corpus callosum Agenesis-linked genes

Total number of genes (26)

Genes with validated SVZ expression (7): DCC, DPYD, FGFR3, NDE1, SASS6, SEMA3C, ZBTB18

\section{Cell division:}

Chromosome segregation

(cell cycle) factors HD, LIM, Myb, ...)
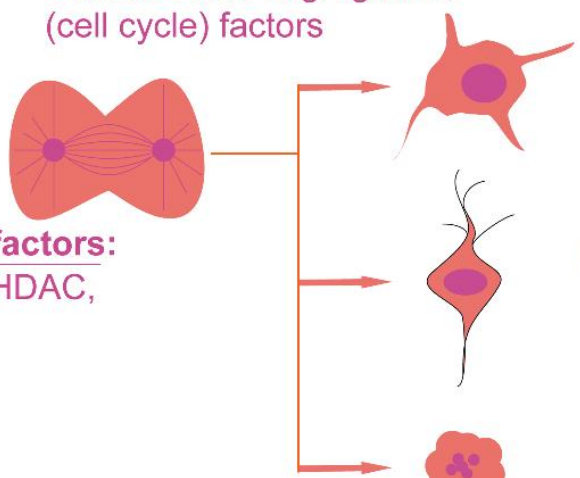

Proliferation:

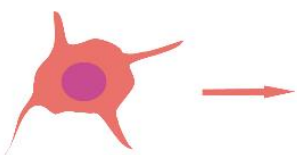

Chromatin \& epigenetic factors: (SWI/SNF, NuRD, PRC2, HDAC, KDM complexes,...)

\section{Signaling factors:}

Hippo, Notch, FoxO, PI3K-Akt, Axon guidance, Fanconi anemia signaling

Cell division cycle factors

Differentiation:

Antiproliferation factors, Neurogenic factors

\section{Apoptosis:}

P53, Caspase signaling 
Figure 2.5 Mutation of IPC Genes May Underlie Human Cortical Malformation and Intellectual Disability (A) Graphical representation of human phenotype ontology for TBR2+ IPCs genes showing the top ten phenotypes and others that follow in ranking. (B, D, and F) List of the genes identified in IPCs with phenotypic implications for intellectual disabilities, microcephaly, and corpus callosum agenesis, respectively. (C, E, and G) Respective array of micrographs showing in situ hybridization of examples of genes (highlighted red in the adjoining gene list) with distinctive expression in the developing mouse cortical subventricular zone, and whose dysfunction can lead to abnormal cortical structure and function. CC, corpus callosum. Magnified cortical region is shown by a red box in (C). Scale bar, $100 \mu \mathrm{m}$. (H) Schema showing examples of regulatory factors involved in transcription regulation, signaling pathways in progenitor cells, and those involved in the cell cycle and chromosome segregation that drive cellular processes such as proliferation, differentiation, and apoptosis.

\subsubsection{Uncovering ESCO2 as a novel IPC-enriched gene essential for SVZ formation and cortical neurogenesis}

Among the novel IPC genes, ESCO2 is in top 100 of IPC-most enriched genes (Table S1). GO analysis also revealed that ESCO2 belongs to top gene categories, including cell cycles, chromatin segregation, transcription regulation, DNA replication and chromatin organization pathways (Table. S2, S4, S5, S6, S7). Mechanistically, ESCO2 and its orthologous ESCO1, encoding for cohesin acetyltransferases, are essential for establishing cohesion between sister chromatids by acetylating the SMC3 subunit of the cohesion ring (Nishiyama et al., 2010; Rolef Ben-Shahar et al., 2008; Unal et al., 2008). In contrast to a highly-enriched expression of ESCO1 in RGCs in VZ (Fig. S2.5A-C), expression of ESCO2 is mostly restricted to IPCs in SVZ (Fig. 2.1H/I, Fig. S2.5D-F). This raises a possibility that ESCO1 and ESCO2 play an important role in biogenesis of RGCs and IPCs, respectively.

To understand the role of selected IPC-specific genes in corticogenesis, we characterized functions of ESCO2 in IPC development. Previous studies has shown that ESCO2 has a critical role in the formation of cortical layers (Whelan et al., 2012b) and its de novo mutations cause primary microcephaly in Roberts-syndrome patients (Vega et al., 2005), suggesting that it might have important, yet undiscovered, roles in the specification and viability of IPCs, and in orchestrating cortical neurogenesis.

To find out the functional significance of ESCO2 during cortical development, we ablated ESCO 2 gene in the early developing mouse cortex using an Emxl-Cre driver (Gorski et al., 2002; Whelan et al., 2012b). Similar to the gradient expression pattern of Emxl, EmxlCer activity is found first in the medial-dorsal cortex (MCX, DCX) at E10.5 and extends to the lateral cortex (LCX) from E12.5 onward (Gorski et al., 2002). Because the Emxl-Cre is active differently in different cortical areas, we first examined the cortical phenotype of ESCO2cKO mutants in MCX and DCX areas (Fig. 2.6A). At E12.5, the ESCO2-ablated presumptive cortex displayed a notable reduction in thickness or size compared with control (Fig. 2.6A). A closer examination revealed a reduction in the population of PAX6+ RGCs in the ESCO2cKO cortex compared with control (Fig. 2.6A/B). Strikingly, the pool of the TBR2+ IPCs is largely lost in MCX and DCX areas of mutants (Fig. 2.6A/B). As indicated by immunostaining for the apoptotic cell marker CASP3, there was overt cell death in the E12.5 ESCO2cKO mutant cortex in the examined cortical areas (Fig. 2.6C).

In accordance with the Emxl-Cre activity, the cortical phenotype of ESCO2cKO mutants appeared mild in LCX than in MCX and DCX (Fig. 2.6A-C). Particularly, the population of PAX6+ RGCs and TBR2+ IPCs were fairly preserved in the cKO LCX (Fig. 
2.6A/B). In addition, the CASP3+ apoptotic cells were found mostly in the basal side of mutant LCX (Fig. 2.6C). Of note, further differential analysis indicated that majority of the cells undergoing apoptosis in the ESCO2cKO LCX were TBR2+ IPCs (Fig. 2.6C-E, empty arrows) and cells in transition stage between RGCs and IPCs (i.e. PAX6+, TBR2+) (Fig. 2.6C-E, filled arrows), albeit other cortical cell types like PAX6+ RGCs and NEUN+ postmitotic neurons also registered apoptotic activity, but to a lesser extent (Fig. 2.6D/E).

Given the reduction in the progenitor pool and death of differentiated neurons, we found drastic decrease in the number of NEUN+ or HUCD+ neurons in the presumptive ESCO2cKO cortex compared with control (Fig. S2.6). Our observations are consistent with previous studies, which reported that cortical layers are not formed as a result of ESCO2 abolishment (Whelan et al., 2012b).

Together, this part of our investigation shows that ESCO2 is expressed in a subset of PAX6+ RGCs and TBR2+ IPCs in the developing cortex. The expression of ESCO2 is required for the viability of these cell populations and their progenies to afford proper cortical histogenesis. 
A Cortex-specific Emx1-Cre

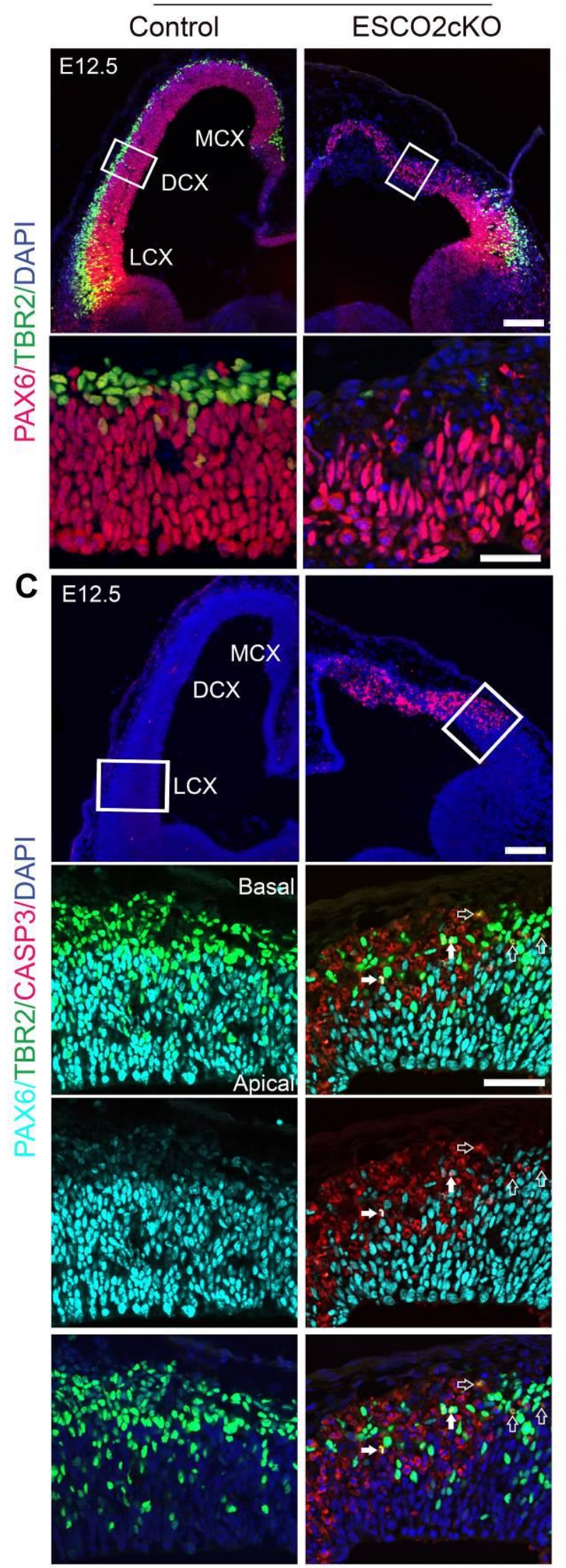

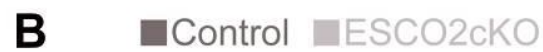

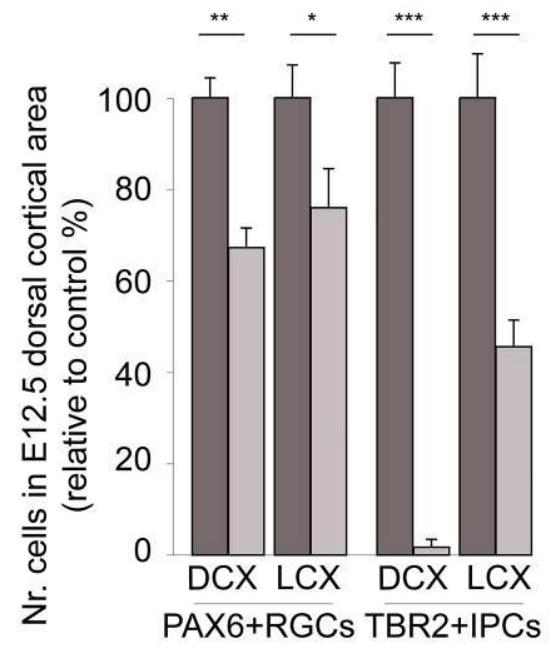

D

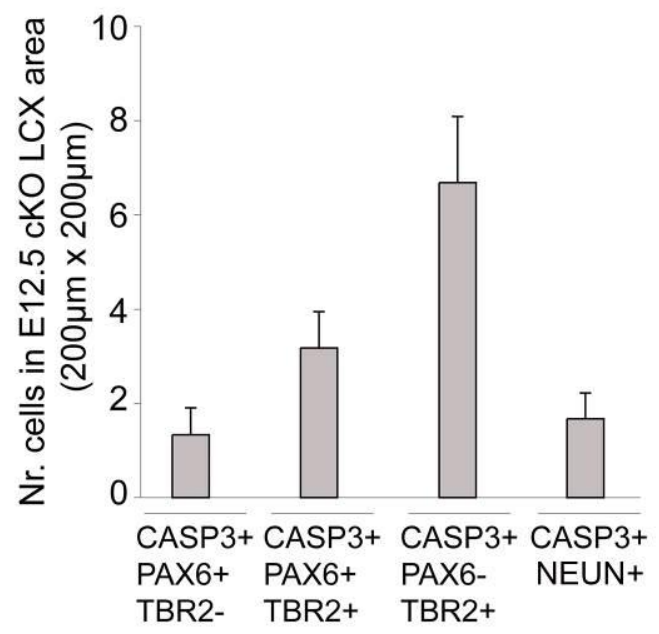

E $-\mathrm{CASP} 3+\mathrm{NEUN}+$

- CASP3+/PAX6-/TBR2+

I CASP $3+/ \mathrm{PAX} 6+/ \mathrm{TBR} 2+$

- $\mathrm{CASP} 3+/ \mathrm{PAX} 6+/ \mathrm{TBR} 2-$

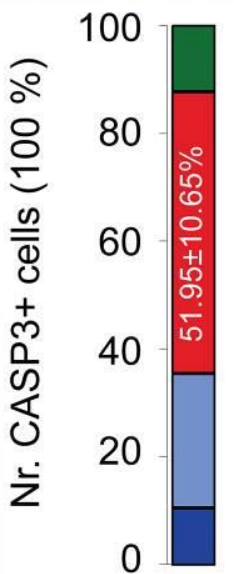

Figure 2.6 Lack of ESCO2 Causes Apoptosis of Cortical Progenitors Leading to Disturbance of Cortical Development

(A) Micrographs showing low and high magnification of the E12.5 control (wild-type) and Esco2 cKO cortex immunostained for PAX6 and TBR2. The medial (MCX), dorsal (DCX), and lateral (LCX) aspects of the cortex 
are indicated. Counterstaining was done with DAPI. (B) Bar graph showing quantification of PAX6+ and TBR2+ cells in the E12.5 control and Esco2 cKO dorsal cortical area marked with a white box in (A). (C) Micrographs showing low and high magnification of the E12.5 control and Esco2 cKO cortex immunostained for PAX6, TBR2, and the apoptosis marker CASP3. Counterstaining was done with DAPI. The medial (MCX), dorsal (DCX), and lateral (LCX) aspects of the cortex are indicated. The basal and apical sides of the cortex are shown. Filled arrows point to PAX6+/TBR2+ cells, which are in transition stage between RGCs and IPCs, undergoing apoptosis (CASP3+), whereas empty arrows point to apoptotic (CASP3+) TBR2+ IPCs. (D and E) Bar graph (D) showing quantification of the number of PAX6+, TBR2+, PAX6/TBR2+, or NEUN+ cells undergoing apoptosis, and composite bar graph (E) showing the quantitative proportion of CASP3+ cells co-expressing PAX6 or TBR2 or NEUN in the lateral cortex (marked with white box in C). Error bars are SEM. $* \mathrm{p}<0.05, * * \mathrm{p}<0.01, * * * \mathrm{p}<0.001$. Experimental replicates (n) $=4$ (B) and 6 (D and E). Scale bars, $50 \mu \mathrm{m}$ (lower panels in A and C) and $200 \mu \mathrm{m}$ (upper panels in A and C).

\subsubsection{ESCO2 is required for maintenance of the IPC population in developing cortex}

Because the loss of ESCO2 in early cortical progenitors in transgenic ESCO2cKO mutants caused massive apoptosis and cortical dysgenesis, we were limited in examining the role of ESCO2 at later stages of cortical development. Alternatively, in utero electroporation (IUE) technique was employed to acutely delete $\mathrm{ESCO} 2$ from individual RGCs in the developing mouse cortex. The prominent expression of ESCO2 in IPCs and cells in the transition between RGCs and IPCs as well as the massive loss of these cell types following ablation of ESCO2 in the early developing cortex in ESCO2cKO embryos, prompted us to investigate whether ESCO2 influences the cell viability and generation of TBR2+ IPCs from RGCs in late corticogenesis.

The brains of ESCO2fl/fl embryos at E15.5 were electroporated either with pCIG2-Creires-GFP (Cre-GFP) or control pCIG2-ires-GFP (GFP) plasmids. The cortices were harvested 30 hours post-electroporation (i.e., at E16.5) and processed for immunohistological analyses (Fig. 2.7A/C). At mid-gestation, RGCs undergo only one division in less than 24 hours to produce daughter cells mainly IPCs in the developing mouse cortex (Noctor et al., 2004). To study the viability of AP daughter cells and the generation of IPCs from RGCs after deletion of ESCO2 in the VZ, we performed triple immunostaining for GFP/PAX6/CASP3 and for GFP/TBR2/CASP3 at E16.5 (Fig. 2.7A/C).

The electroporated (eGFP+) cells mainly occupied the $\mathrm{VZ}$ and the basal half of the cortical wall (i.e., SVZ and IZ). In contrast to almost no CASP3+ cells found in control (GFP) plasmid-injected cortex, many CASP3+ apoptotic cells were seen in Cre-electroporated cortex as expected (Fig. 2.7A/C). In the Cre-injected cortex, majority of the GFP+/CASP3+ cells were found to be either negative $(\sim 80.0 \pm 11.4 \%)$ or low $(\sim 17.6 \pm 5.4 \%)$ for PAX6 expression (Fig. $2.7 \mathrm{~A} / \mathrm{B})$. On the other hand, the number of GFP+/CASP3+ cells expressing TBR2 was much higher $(78.0 \pm 9.7 \%)$ than those without TBR2 expression (Fig. 2.7C/D). The findings further support the idea that expression of ESCO2 is required for TBR2+ IPC viability and those of committed RGCs (with low PAX6 expression) to generate IPCs in both early and late cortical development.

Interestingly, there was no significant difference between the GFP- and Cre-GFPelectroporated cortex in terms of the number of transfected cells (GFP+) expressing PAX6 or TBR2 (Fig. 2.7E). Thus, ESCO2 is dispensable for the differentiation of RGCs, which are low in ESCO2 expression (Fig. 2.7F, Fig. S2.5), into IPCs. It implies that following the acute deletion of ESCO2, IPC as progenies of PAX6-expressing RGCs are likely normally formed 
but fail to survive. It is also conceivable that NEUN+ or HUCD+ neurons that manage to differentiate from the ESCO2-deficient cortical neural progenitors, especially IPCs, are unhealthy and subsequently die via apoptosis (Fig. 2.7F, Fig. S2.6).

Taking our data together, we show that ESCO2 expression is essential for the maintenance of IPCs and proper neurogenesis during cortical development.

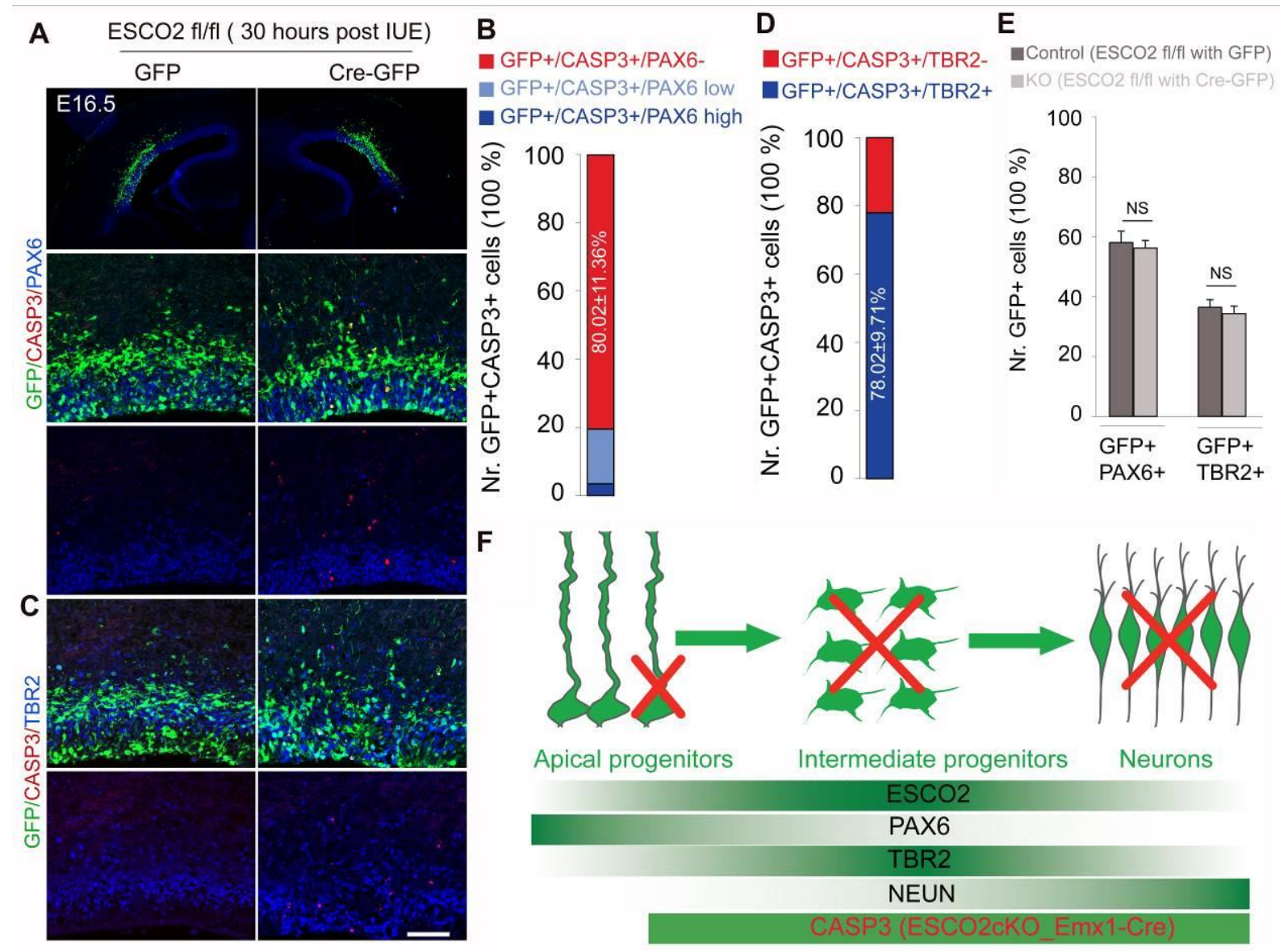

Figure 2.7 Expression of ESCO2 Is Important for Maintenance but Not Generation of IPCs

(A and C) Micrographs at low and high magnification showing GFP, CASP3, and PAX6 (A) or TBR2 (C) immunostaining in the E16.5 Esco2fl/fl mouse cortex electroporated with a GFP-only plasmid as control and GFPCre plasmid to delete Esco2 in the transfected cells. (B and D) Composite bar graphs showing quantitative analysis of the proportion of GFP and CASP3 positive cells with either no/low/high PAX6 expression (B) or with/without TBR2 expression (D). (E) Bar graphs showing no significant difference between the total number of cells coexpressing GFP and PAX6 or GFP and TBR2 when the control (GFP-only) and knockout (GFP-Cre) cortices are compared. Error bars are SEM. (F) Schema illustrating the expression of PAX6, TBR2, NEUN, and ESCO2 during differentiation of radial glial progenitors to intermediate progenitors and neurons. The loss of ESCO2 in Esco2cKO_Emx1-Cre seems to cause apoptosis in the late radial glial progenitors (PAX6+/TBR2+), intermediate progenitors (TBR2+), and neurons (NEUN). NS, not significant. Experimental replicates (n) $=6$ (B, D, E). Scale bar, $50 \mu \mathrm{m}$.

\subsection{Discussion}

Transcriptome analyses of molecularly sorted cells can enrich the identification of celltypespecific factors, which can help us understand the molecular landscape in cell lineages. In this study, we report the molecular characterization of the evolutionarily and clinically important population of IPCs in the developing mouse cortex. We identified distinct sets of largely uncharacterized genes that exhibit enriched expression in IPCs among other cell types in the 
developing cortex. The set of genes were found to belong to transcription regulators, chromatin and epigenetic factors, signaling factors, and chromosome segregation (cell cycle) regulators. These genes encode critically important molecules for proper proliferation, differentiation and maintenance of IPCs. Even though our understanding of the contribution of IPCs in cortical development has improved, several key questions remain enigmatic (Hevner, 2019). Our study represents the first comprehensive characterization of the molecular signature of IPCs in developing mouse cortex. The findings provide hints for future investigation to resolve the many unanswered questions.

Previous studies indicate that more than half of the IPC daughter cells undergo apoptosis during corticogenesis (Hevner, 2019; Mihalas and Hevner, 2018). The relevance of this observed phenomenon is undetermined, however, it might associate with the regulation of the net neurogenic output, genome quality, neuronal subtype proportions during cortical development and cortical evolution (Haydar et al., 1999; Hevner, 2019). The observed high abundance of apoptotic cells among IP daughter cells harmonizes with previous reports documenting marked cell death in the SVZ and IZ of embryonic rodent cortex (Blaschke et al., 1996; Thomaidou et al., 1997). In the same line of evidence, our GO analysis revealed that genes belonging to the caspase cascade in apoptosis are enriched in IPCs. Remarkably, disruption of the caspase cascade leads to decreased programmed cell death resulting in neuronal supernumerary, which likely accounts for the expansion and exencephaly of the forebrain, cerebral gyrification (Kuida et al., 1998; Kuida et al., 1996). Conversely, dysregulation of chromosomal segregation can cause an increase in neural progenitor cell death leading to loss of neurons as exemplified in our ESCO2 case study. Thus, the proper coordination of the various aspects of the apoptotic signaling pathway, especially during embryonic neurogenesis, is essential for the determination of normal cortical size and form. Given the critical contribution of apoptosis to correct progression of cortical morphogenesis, it would be of great interest for future investigations to elucidate the precise mechanisms that trigger apoptotic cell death of neural cells during cortical development.

Notably, our validation investigations revealed that lack of ESCO2, one of the identified IPC-enriched genes, results in striking loss of IPC population, leading to the failure of proper formation of the cortex. By using different model systems such as yeast, primary mouse embryonic fibroblasts and human cells (HeLa and 293T human embryonic kidney cells), previous studies reported that ESCO2 is crucial for sister chromatid tethering (Hou and Zou, 2005; Terret et al., 2009; Vega et al., 2005; Whelan et al., 2012a; Whelan et al., 2012b). It is known to do so via its catalytic function in cohesin acetylation that ensures proper cohesion between sister chromatids. Indeed, dysfunction of ESCO2 has been shown to result in loss of cohesion at heterochromatic regions of centromeres, leading to defective localization of cohesin on chromosomes and apoptosis (Hou and Zou, 2005; Terret et al., 2009; Vega et al., 2005). In developing mouse cortex, a highly-enriched expression of ESCO1 and ESCO2 was found in RGCs in VZ and in IPCs in SVZ, respectively. This suggests a possibility that the cohesion acetyltransferases ESCO1 and ESCO2 are key cell viability factors, which act by maintaining the appropriate cohesion in pericentric heterochromatin in RGC and IPC populations. Indeed, our findings indicate that ESCO2 is indispensable for IPC maintenance and demonstrate the identification of a central genetic determinant of IPC biogenesis in the developing mouse cortex. 
In conclusion, our transcriptome data provide a crucial resource for further investigations aimed at understanding how IPC-related genetic factors contribute to cortical development and their implication for neurological disorders. Moreover, because IPCs are believed to be responsible for a large portion of mammalian corticogenesis, and the size of the IPC-laden SVZ correlates with brain phylogeny, future studies can look into the role of the identified IPC genes in cortical evolution.

\subsection{Experimental procedures}

\subsubsection{TBR2+ nuclei sorting protocol for transcriptomic data generation from embryonic mouse brain}

Cells expressing TBR2 in the E16.5 mouse cortex were isolated by FACS and profiled using RNA sequencing. The detailed protocol is reported in Sakib et al. (2021). The experiment was carried out using three biological replicates

\subsubsection{RNA sequencing and bioinformatics analysis}

Sorted nuclei were collected into NSB coated falcon tubes, pelleted with brief centrifugation and the RNA was isolated using Trizol LS (Invitrogen) protocol along with aqueous phase cleanup using Zymo RNA clean \& concentrator-5 kit. RNAseq libraries were prepared using Takara SMART-Seq v4 Ultra Low Input RNA kit using 1ng of RNA according to the manufacturer's protocol. Base calling, fastq conversion, quality control, and read alignments were all performed as outlined previously (Narayanan et al., 2015; Nguyen et al., 2018). Reads were aligned to mouse genome mm10 and counted using FeaturesCount (http://bioinf.wehi.edu.au/featureCounts/). Further descriptions of informatics analyses are provided in supplemental experimental procedures.

\subsubsection{Transgenic mice and in utero electroporation (IUE)}

Floxed ESCO2 (Whelan et al., 2012b) and Emxl-Cre (Gorski et al., 2002) mice were maintained in a C57BL6/J background. Animals were handled according to the German Animal Protection Law. In utero electroporation was performed as described previously (Tuoc et al., 2013; Tuoc and Stoykova, 2008).

\subsubsection{Plasmids and antibodies}

A list of plasmids and antibodies with detailed descriptions is provided in the supplemental experimental procedures.

\subsubsection{Immunohistochemistry (IHC) and in situ hybridization (ISH) validation}

IHC and ISH were performed as previously described (Bachmann et al., 2016; Tuoc et al., 2013; Visel et al., 2004). Briefly, sections for IHC were incubated overnight with primary antibody at $4{ }^{\circ} \mathrm{C}$ after blocking with normal sera of the appropriate species. Primary antibodies were detected with a fluorescent secondary antibody (Alexa Fluor, 1:400; Invitrogen). Sections were 
later counterstained with Vectashield mounting medium containing DAPI (Vector laboratories) to label nuclei.

A detailed ISH protocol with different conditions was described in our previous study (Visel et al., 2004) and can be found in our online digital atlas (https://gp3.mpg.de). The template sequence and ISH condition are described in webpage for each gene.

\subsubsection{Imaging, quantification, statistical analysis and data availability}

Micrographs were obtained by confocal fluorescence microscopy (TCS SP5, Leica) and analyzed using an Axio Imager M2 (Zeiss) with a Neurolucida system. Images were processed further using Adobe Photoshop. The statistical quantification was carried out as average from at least three biological replicate. Detailed statistical analyses and description for histological experiments are presented in Table S8. The in situ expression of all the identified IPC genes are freely available online (https://gp3.mpg.de) in an interactive database.

\subsection{Accession numbers}

All RNA-seq data have been deposited in the Gene Expression Omnibus under accession number GEO: GSE168298.

\subsection{Author contributions}

MSS, CK and AF established TBR2+ cell sorting and RNA-Seq. PAU, LP, GS, YX, JR, XM analyzed RNA-seq data and mouse phenotype. PD, GE contributed in generation of ESCO2cKO line, histological analysis and provided ISH data. JFS, GE, AF, HPN provided research tools and offered discussions for the study; TT conceived the study; TT and GS wrote the manuscript.

\subsection{Declaration of interests}

The authors declare no competing interests.

\subsection{Acknowledgements}

We acknowledge U. Kunze, C. Heuchel for their expert animal care. We thank K. Jones for providing reagents, M. Witte for helpful discussions. This work was supported by TU432/1-1, TU432/3-1, TU432/6-1 DFG grants (TT), Schram-Stiftung (TT). AF was supported by the DZNE, the ERC consolidator grant DEPICODE (648898), the SFB1286 and the DFG under Germany's Excellence Strategy-EXC 2067/1390729940.

\subsection{Supplemental figures}


A

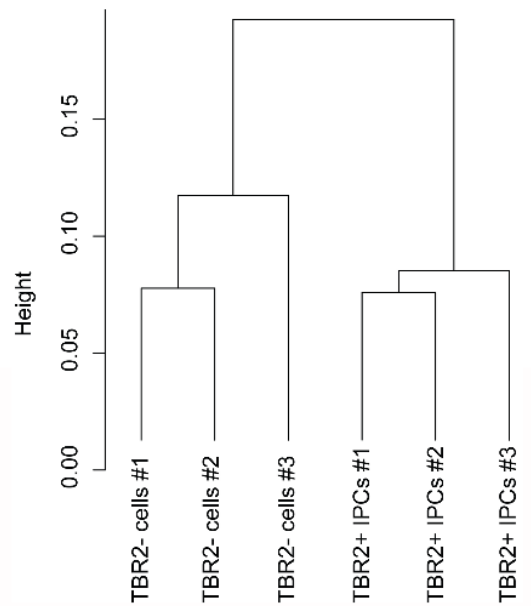

B

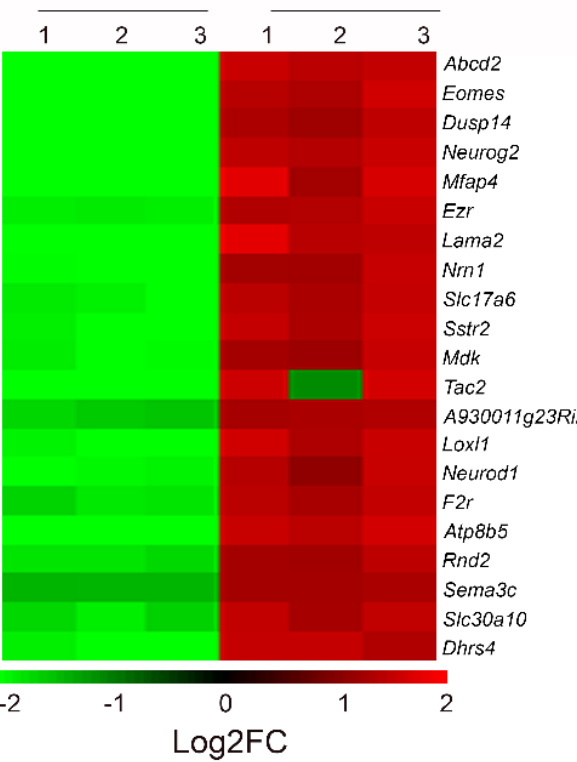

D This study $(P<0.01, \log 2 F C>1.0)$

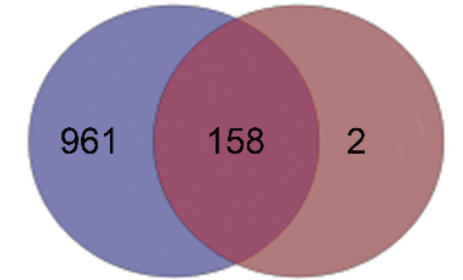

scRNA-seq (Kawaguchi et al. 2008;

Telley et al. 2016; Loo et al. 2019, Li et al., 2020)
C 5.0

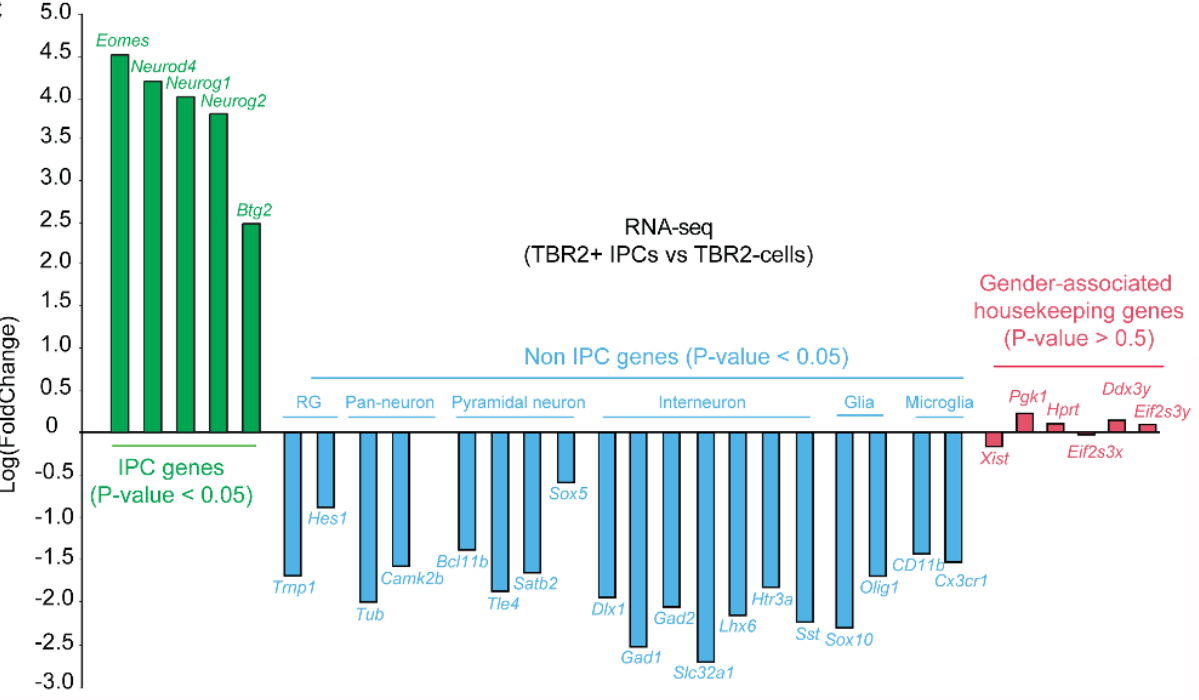

E

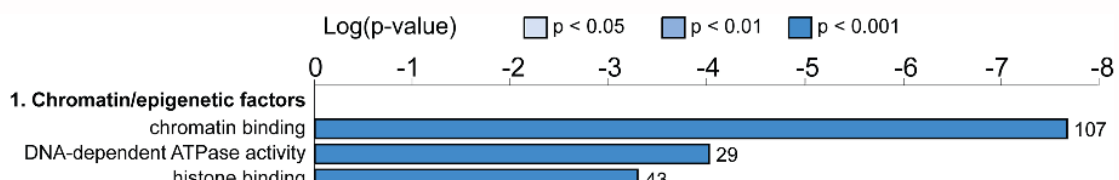
histone activity

helicase activity

nucleosome binding

DNA helicase activity

2. DNA binding factors

double-stranded DNA binding

chromatin DNA binding

transcription coregulator activity

nucleoside binding

ribonucleotide binding

regulatory region nucleic acid binding

transcription factor binding

ulatory region DNA binding

nucleosomal DNA binding

pecific double-stranded DNA binding

3. RNA binding factors

ribonucleoside binding 258

mRNA binding

MRA 3'-UTR binding

slational modifications

4. Post-translational modifications

$\begin{aligned} \text { ubiquitin-like protein ligase binding } & \\ \text { ubiquitin protein ligase binding } & \\ \text { Kinase } & \end{aligned}$

kinase binding

protein kinase binding

Deacetylase activity

protein deacetylase activity

deacetylase activity

Figure S2.1 (related to Figure 2.1). RNA-seq analysis of mouse TBR2+ IPCs and TBR2-cells.

(A) Cluster dendrogram analysis of RNA-seq for TBR2+ and TBR2-sorted cell samples. (B) Heatmap showing the top 20 genes with high expression (enriched/upregulated) in TBR2+ IPCs and low expression in TBR2-cells. (C) Bar graph showing expression of selected IPC (in green), non-IPC (in blue) and gender-associated housekeeping genes (in red) in IPCs (TBR2+) and non IPCs (TBR2-) in the E16.5 mouse cortex. (D) An indication of the increased number of IPC-enriched genes identified in our study compared with previous studies performed at the IP single cell level. (E) Bar graph showing the molecular pathway analysis in TBR2+ IPCs and the number of genes belonging to each category of pathway. 

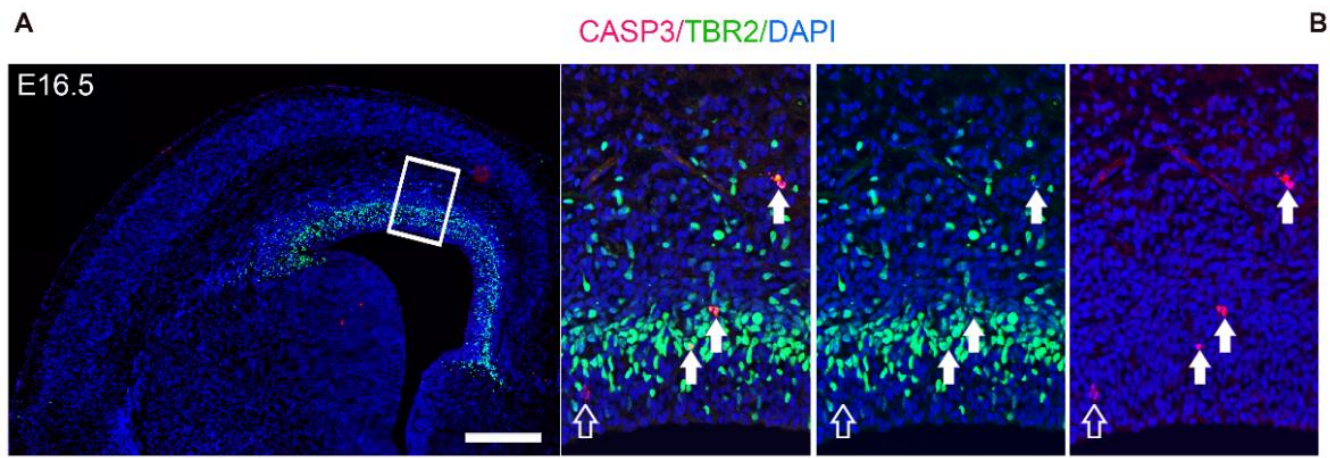

口CASP3+/TBR2 ICASP3+/TBR2

Figure S2.2 (related to Figure 2.3). TBR2+ IPCs in mouse display prominent apoptotic activity.

(A) Immunohistochemical micrographs showing an overview of E16.5 mouse cortex and highly magnified germinal zone stained with TBR2 and CASP3 antibodies. The zoomed area is indicated with white inserted box. Filled arrows point to TBR2+ IPCs undergoing apoptosis whereas empty arrow indicates apoptotic activity in a TBR2-cell. Counterstaining was done with DAPI. (B) Bar graph showing the proportion of TBR2+ and TBR2cells undergoing apoptosis. Experimental replicates $(n)=6$ (B). Scale bar $=100 \mu \mathrm{m}$.

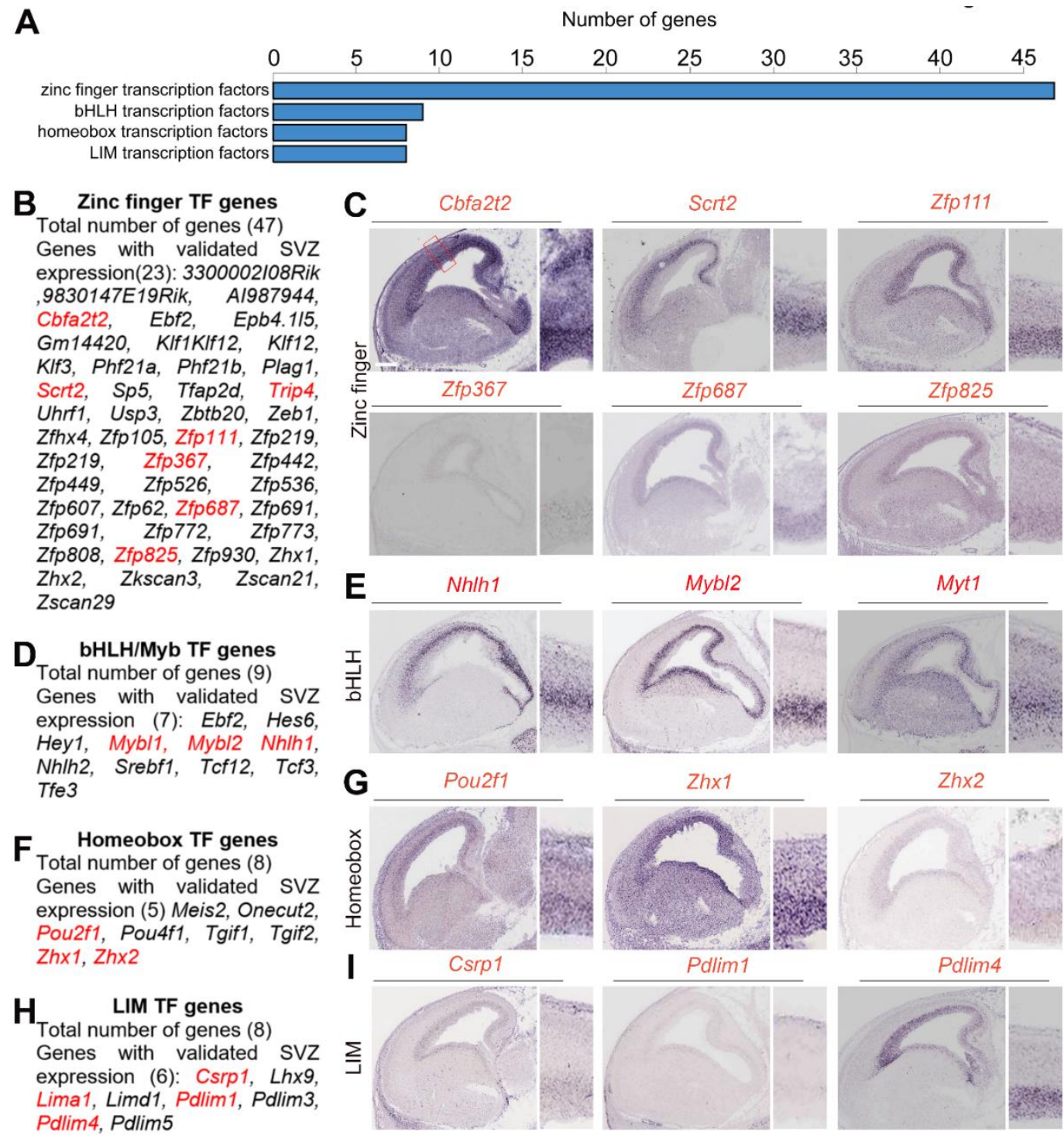

Figure S2.3 (related to Figure 2.4). Enrichment of transcription factor genes in TBR2+ IPCs.

(A) Bar graph showing the number of genes in IPCs under the indicated classes of transcription factor. (B, D, F, H) Categories of transcription factors with corresponding list of newly identified IPC-enriched genes. (C, E, G, I) Respective micrographs showing in situ hybridization of examples of the identified transcription factor genes (highlighted red in the adjoining gene list) with expression endowment in the developing mouse cortical subventricular zone. Magnified cortical region is shown with red box in (C). Scale bar $=100 \mu \mathrm{m}$. 


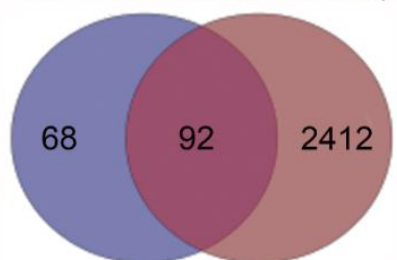

ScRNA-seq for developing human cortex (Pollen et al., 2015; Nowakowski et al. 2017; Fan et al. 2018; Li et al. 2018; Zhong et al. 2018) Fan et al. 2018; Li et al. 2018; Zhong et al. 2018)

C

$5.0 \quad$ Mfap4 Human bIP genes with their altered expression 4.5 Eomes Neurod4 $_{4}$ in mouse TBR2+ IPCs vs mouse TBR2- cells
B Bulk RNA-seq for mouse TBR2+ IPCs This study $(P<0.01, \log 2 F C>1.0)$

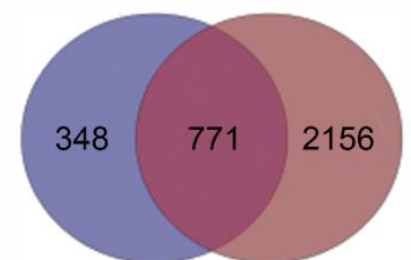

scRNA-seq for developing human cortex

(Pollen et al., 2015; Nowakowski et al. 2017;
D
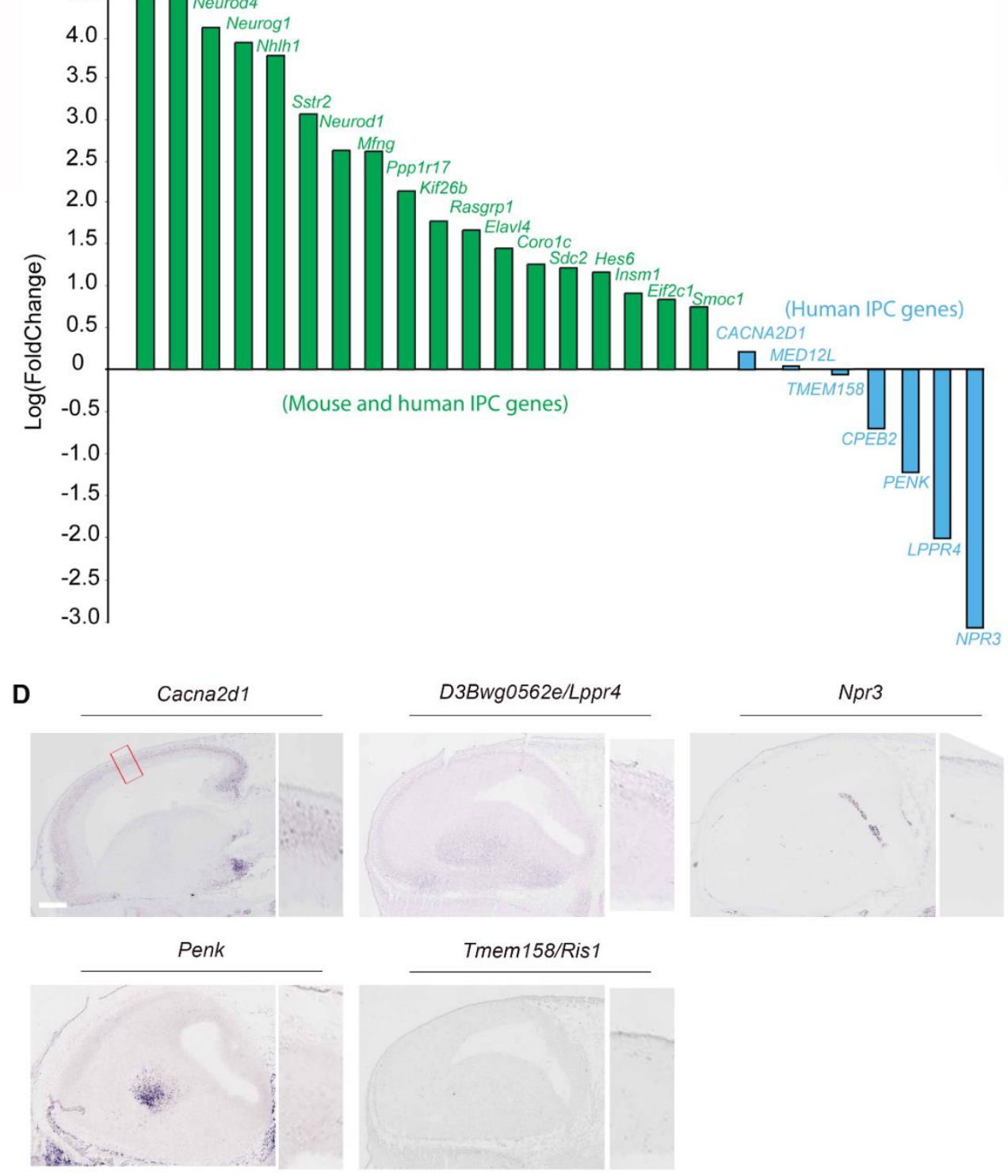

Figure S2.4 (related to Figure 2.5). Many human bIP genes are upregulated in mouse TBR2+ IPCs while others are downregulated or absent.

(A, B) Overlap between the number of human IPC genes from scRNA-seq analysis of the human developing cortex (Fan et al., 2018; Li et al., 2018a; Nowakowski et al., 2017; Pollen et al., 2015; Zhong et al., 2018) and number of mouse IPC genes, which was recently identified by scRNA-seqanalysis (Kawaguchi et al., 2008; Li et al., 2020b; Loo et al., 2019; Telley et al., 2016) (A), and by bulk RNA-seq (B, this study). (C) Bar graph showing both upregulated (enriched) and downregulated human bIP genes in TBR2+ IPCs compared with TBR2- cells in mouse cortex. (D) In situ hybridization micrographs showing the E14.5 mouse cortex riboprobed for the indicated human bIP genes. Magnified cortical region is shown with red box. Scale bar $=100 \mu \mathrm{m}$. 


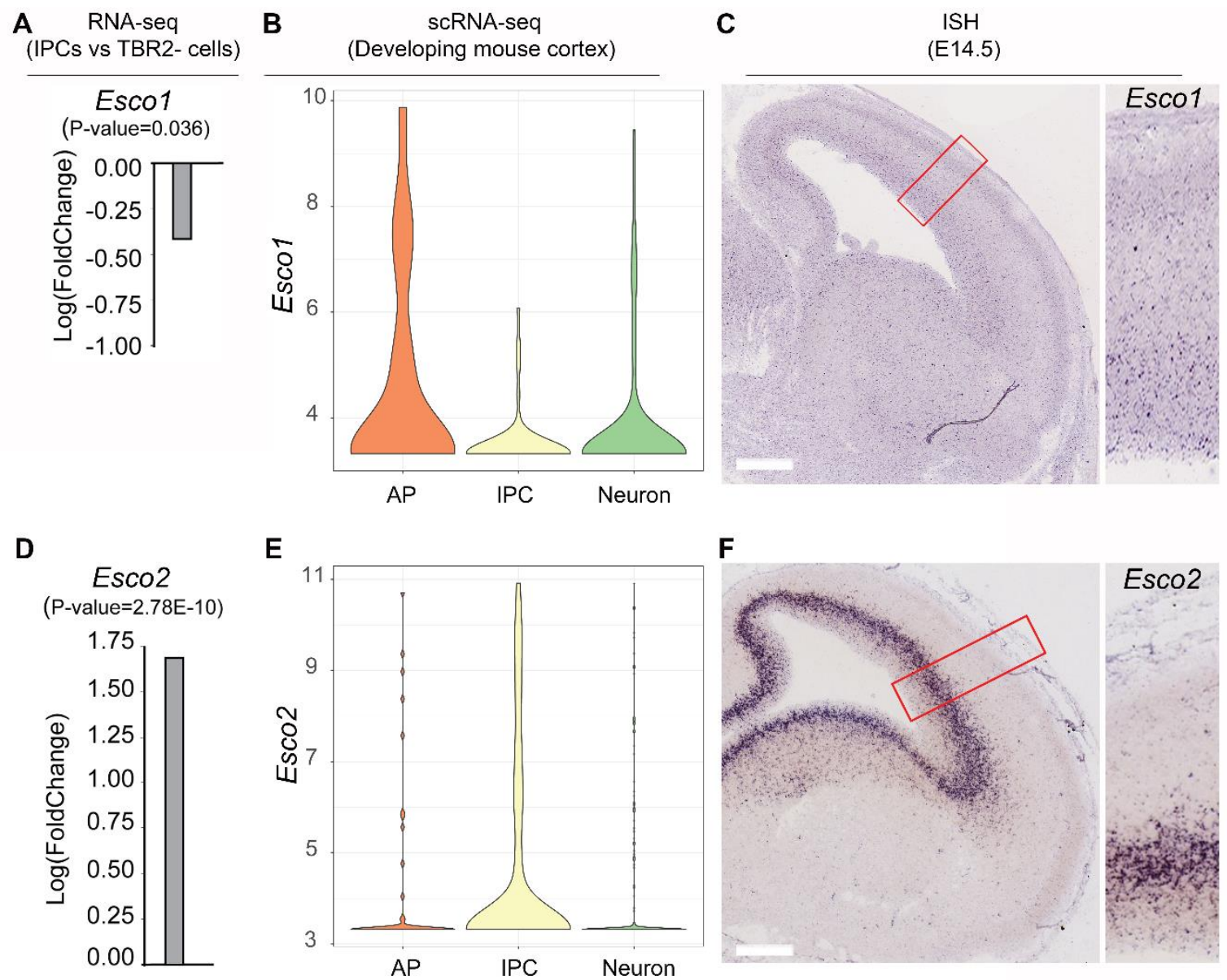

Figure S2.5 (related to Figure 2.6). Expression of ESCO1 and ESCO2 in the developing mouse cortex.

(A-F) Expression of ESCO1 (A-C) and ESCO2 (D-F) were evaluated by RNA-seq with TBR2+ and TBR2samples (A, D), single-cell (sc)RNA-seq (B, E), and ISH (C, F). (A, D) Bar graph indicating significant differential expression of ESCO1 (A) and ESCO2 (D) in TBR2+ IPCs compared with TBR2- cells in RNA-seq analysis. (B, E) Expression of Esco1 (B) Esco2 (E) based on a published single-cell scRNA-seq dataset of the developing mouse cortex (Telley et al., 2016). The graph-plots were generated using the Seurat package of R (Macosko et al., 2015) (http://genebrowser.unige.ch/science2016/). (C, F) Micrograph of in situ hybridization (ISH) staining showing prominence of Escol (C) and Esco2 (F) expression in VZ and SVZ of E14.5 mouse cortex, respectively. Magnified cortical region is shown with red box. Note that in contrast to a highly-enriched expression of Escol in RGCs in VZ, expression of Esco2 is mostly restricted in IPCs in SVZ. Abbreviations: RGC (Radial glial progenitor cell), IPC (Intermediate progenitor cell). Scale bar $=100 \mu \mathrm{m}$. 
A Cortex-specific Emx1-Cre

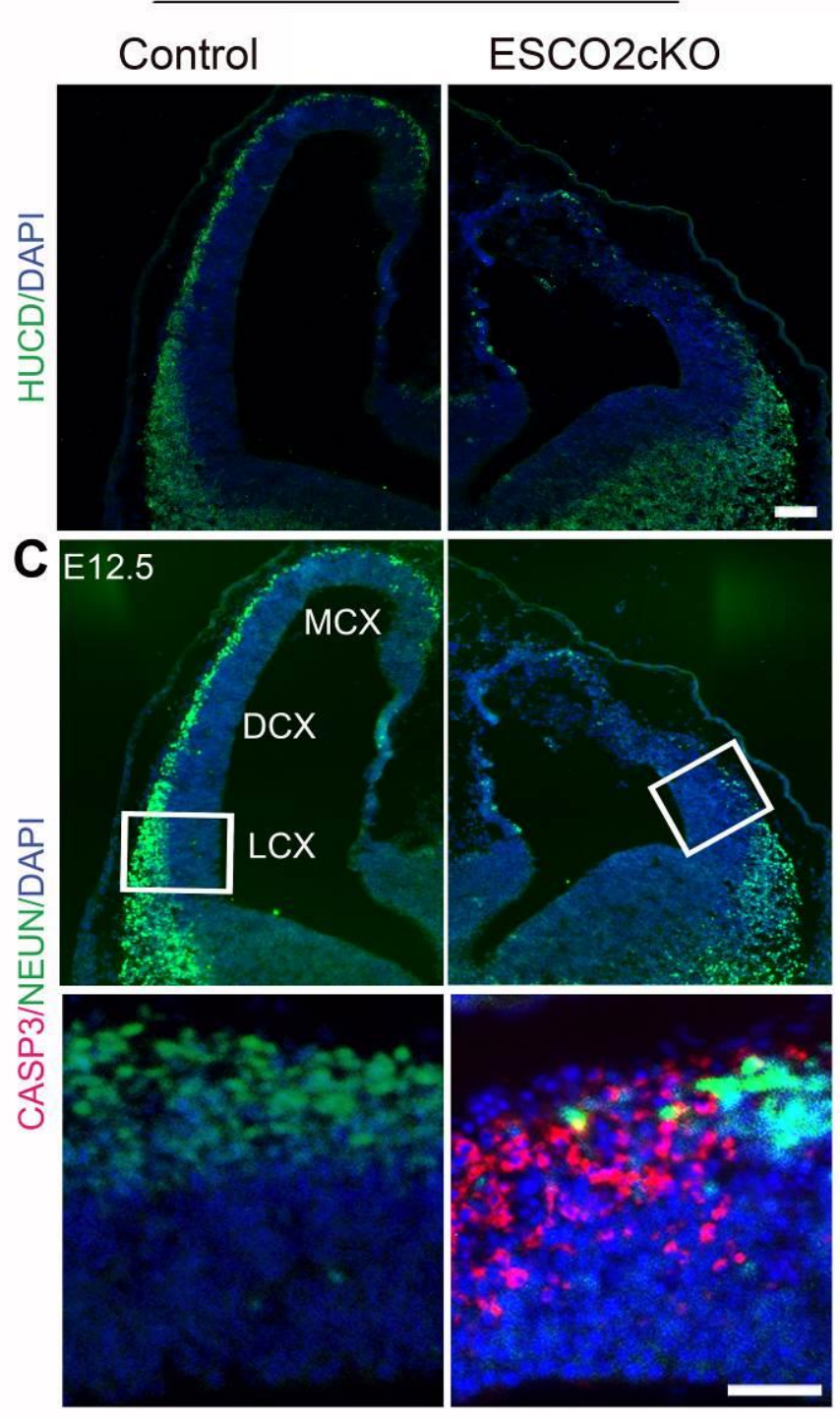

B EControl

口ESCO2CKO

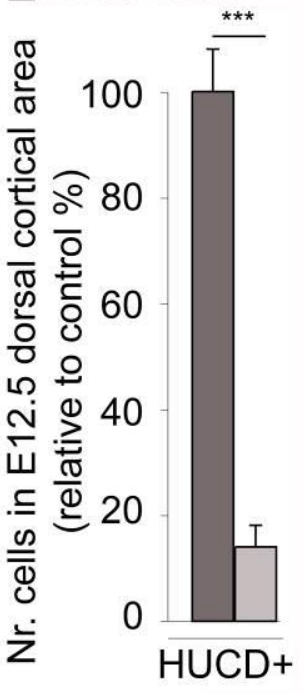

D

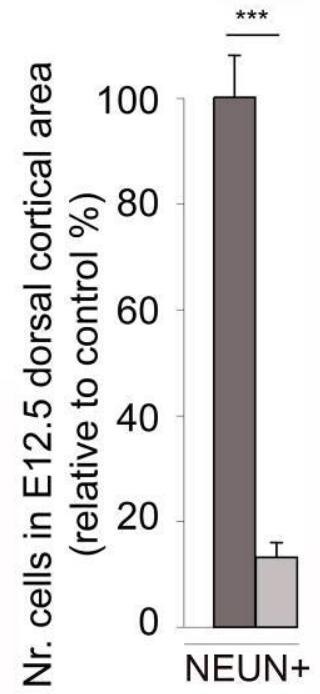

Figure S2.6 (related to Figure 2.7). Deletion of ESCO2 in developing cortex causes depletion of post-mitotic neurons via apoptosis.

(A, C) Immunohistochemical micrographs showing staining of the E12.5 mouse cortex with the antibodies HUCD and NEUN (pan-neuronal markers), and CASP3 to mark post-mitotic neurons undergoing apoptotic cell death. (B, D) Bar charts showing quantification of the number of HUCD+ and NEUN+ cells in the lateral aspect of the E12.5 cortex (marked with white box). The medial (MCX), dorsal (DCX), and lateral (LCX) cortical areas are indicated. *** $p$-value $<0.001$, Experimental replicates $(\mathrm{n})=4(\mathrm{~B}, \mathrm{D})$. Scale bar $=200 \mu \mathrm{m}(\mathrm{A}), 50 \mu \mathrm{m}(\mathrm{C}$, lower panel).

\subsection{Supplemental tables}

Supplemental tables S1-8 can be found online at https://doi.org/10.1016/j.stemcr.2021.03.008.

\subsection{Supplemental experimental procedures}

\subsubsection{Plasmids}

Plasmids used in this study: CAG-GFP-IRES-CRE (Zhao et al., 2006) from Addgene. 


\subsubsection{Antibodies}

The following polyclonal $(\mathrm{pAb})$ and monoclonal $(\mathrm{mAb})$ primary antibodies used in this study were obtained from the indicated commercial sources: CASP3 rabbit pAb (1:100; Cell Signaling), ESCO2 (Whelan et al., 2012), GFP chick pAb (1:400; Abcam), HUCD mouse mAb (1:20; Invitrogen), NEUN mouse mAb (1:200, Chemicon), PAX6 mouse mAb (1:100; Developmental Studies Hybridoma Bank), PAX6 rabbit pAb (1:200; Covance), TBR2 rabbit pAb (1:200; Abcam), TBR2 rat 923 mAb (1:200; eBioscience).

Secondary antibodies used were Alexa 488-, Alexa 568-, Alexa 594- and Alexa 647- conjugated IgG (various species, 1:400; Molecular Probes).

\subsubsection{Functional enrichment analysis of IPC genes}

The lists of IPC genes ( $\mathrm{p}$-Value $<0.01$, log2FoldChange $>1.0$ ) were uploaded to the DAVID functional annotation tool (https://david.ncifcrf.gov/). Then, representative enriched biological functional terms were manually selected. To perform the Gene Set Enrichment Analysis (GSEA) analysis, the list of upregulated genes was uploaded to GSEA. Using FDR qvalue $<0.05$ as a cut-off generated the enriched biological and cellular component terms. The Fisher's exact test was applied to identify the terms showing a statistically significant difference for the upregulated genes.

\subsubsection{Identification of IPC-enriched InRNAs}

The lists of IPC genes ( $\mathrm{p}$-Value $<0.01, \log 2$ FoldChange $>1.0$ ) were uploaded to the MGI gene nomenclature analysis tool (http://www.informatics.jax.org/batch). List of lnRNAs proteinencoding genes was extracted from each other.

\subsubsection{Protein-protein interaction network}

The combined list of IPC-enriched genes encoding for TFs (chromatin remodelling and epigenetic factors, Fig. 2.4J) was uploaded to the STRING database (http://stringdb.org/). The protein-protein interactions from STRING were visualized by Cytoscape (https://cytoscape.org/; version: 3.3.0).

\subsection{Supplemental discussion}

\subsubsection{Prominence of cell cycle-related factors in IPCs}

Further unanswered questions that need to be addressed to increase our understanding of ICP cell biology include (1) the reasons behind shorter S-phase and longer G1-phase, and total length of cell cycle in IPCs than in RGCs, and (2) what factors drive the proliferation of some IPCs in cell cycle?. In developing cortex, the length of G1 is increased in neurogenic progenitor cells compared with proliferative progenitors (Caviness et al., 2003; Dehay and Kennedy, 2007; Lukaszewicz et al., 2005; Salomoni and Calegari, 2010). As such, basal progenitors, including IPCs, are known to display a more extended G1 phase than RGCs (Calegari et al., 2005; Salomoni and Calegari, 2010). The increased G1 phase may support the more differentiative 
capacity of IPCs compared with RGCs, thus likely promoting IPC fate (Dehay and Kennedy, 2007; Lange et al., 2009). Indeed, functional manipulation of G1 length was shown to have effects that either support (i) IPC genesis leading to neurogenic division and premature neurogenesis (Calegari et al., 2005) or (ii) increased proliferative divisions, resulting in progenitor pool expansion, which manifests in cortical layer phenotypes later in development (Lange et al., 2009; Pilaz et al., 2009). Of note, we found a high expression of genes encoding for Cdks $(C d k 2, C d k 4)$ with their regulator protein (Ccnd1, Ccnd2, Ccne1, Ccne2), which drive the G1 phase of cycling IPCs. Hence, it is worth to examine the proliferation capacity of IPCs, in which the expression of these G1-phase factors is specifically manipulated.

\subsubsection{Identification of new IPC-specific transcription factors}

An intriguing molecular difference between the TBR2+ IPCs and the TBR2- cells in the developing mouse cortex is the differential expression of genes, which encode for different TF families (Table S5).

The zinc finger TFs form the largest protein family, having a wide range of molecular functions, and are involved in the development and differentiation of several cell lineages (Cassandri et al., 2017). Expression of many genes encoding for this TF family is enriched in IPCs. The role of the zinc finger proteins identified in this study in neurogenesis are largely unknown, except for that reported for the function of Uhrf1 in adult neurogenesis (Blanchart et al., 2018; Murao et al., 2019).

The second largest family of TFs is the bHLH TFs, which play key roles in various developmental processes in organisms from yeast to humans (Jones, 2004). Class I bHLH proteins are ubiquitously expressed, whereas class II bHLH proteins are tissue-specific. The nervous system-specific bHLH factors can further be classified into proneural and neural differentiation genes (Dennis et al., 2019). Two closely-related nescient helix loop helix 1 (Nhlh1/bHLHa35) and 2 (Nhlh2/bHLHa34) genes belong to the neural differentiation

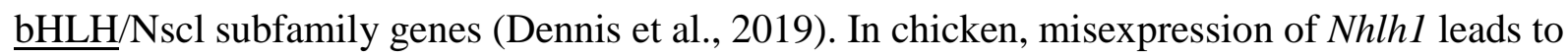
an abnormal brain structure with an underdeveloped cerebellum and a larger tectum caused by changes in cell proliferation ( $\mathrm{Li}$ et al., 1999). Nhlhl-deficient mice exhibit a predisposition to arrhythmia leading to an early death due to autonomic nervous system dysfunction (Cogliati et al., 2002). The phenotype was more severe when Nhlhl-knockout mice were also heterozygous for Nhlh2. The specific and high expression of Nhlh1 and Nhlh2 in IPCs (Table. S5) suggests that the two factors act together to control the IPC differentiation. Expression of other bHLH TFs, including Hes6/bHLHb41, Tcf3/bHLHb21, Tcf12/bHLHb20, Hey1/bHLHb31, and Ebf2, Srebf1/bHLHd1 were found to be enriched in IPCs. Among them, Hes6, Tcf3, Tcf12, Heyl and $E b f 2$ were reported to be involved in neural development (Chuang et al., 2011; Gribble et al., 2009; Methot et al., 2013; Nam et al., 2016; Sakamoto et al., 2003; Uittenbogaard and Chiaramello, 2002; Yang et al., 2015). For Srebf1, no studies have yet been published on its role in neural development, albeit associations with Schizophrenia and Parkinson's disease have been reported, making a future investigation into its role in brain development even more interesting (Le Hellard et al., 2010; Lou et al., 2019; Yang et al., 2016).

The homeobox TFs were also found to be highly expressed in IPCs. Homeobox genes are important for the embryonic development of diverse animals, and are often comparatively 
analyzed to investigate evolution of animal development (Holland et al., 2007). Our investigation of genes enriched in IPC revealed three transcription factors that belong to the homeobox gene family. For two of the homeobox genes enriched in IPC, Meis homeobox 2 (Meis2) and POU domain, class 4, transcription factor 1 (Pou4f1, also called Brn3a), a role for neural development has already been described. MEIS2 has been described as a regulator of dorsal midbrain development interacting with the paired-box transcription factors PAX3 and PAX7 (Agoston et al., 2012). In humans, MEIS2 mutations can cause intellectual disability (Douglas et al., 2018; Giliberti et al., 2019; Louw et al., 2015). In the developing nervous system, Pou4fl was shown to be essential for the generation of dorsal root ganglia sensory neurons and the regulation of sensory afferent projections (Zou et al., 2012). The other homeobox gene is the one cut domain family member 2 (Onecut2), which is well known as a master regulator in cancer (Lu et al., 2018; Rotinen et al., 2018). Function of ONECUT2 in brain development has not yet been described, however, Onecut 2 overexpression was shown to induce a neuron-like morphology and neuronal gene expression in fibroblasts making its role in neural development plausible (van der Raadt et al., 2019).

Another group of TF genes found to be enriched in IPC lineage is the myeloblastosis oncogene-like (Myb-like) transcription factor. The transcription factor MYBL1 (also called AMYB) is known as a master regulator of meiosis (Bolcun-Filas et al., 2011), and in mice, it plays a vital role in spermatogenesis and mammary gland development (Toscani et al., 1997). Although Mybll expression in neuronal progenitor cells has already been described (Trauth et al., 1994), its specific role in brain development is far from clear. Similarly, another Myb-like transcription factor, named MYBL2 or B-MYB was found to be enriched in IPC. MYBL2 is involved in cell proliferation and survival, however, these roles have been investigated mainly in cancer research and a possible function in neurogenesis has not been determined so far (Chen and Chen, 2018; Musa et al., 2017).

Altogether, our investigation of genes specific to IPCs revealed the expression of many transcription factors that were previously not known and, thus, are putative genetic determinants of this cohort of neuronal progenitor cell type. Understanding the function of these IPC-enriched TFs would not only shed light on the mechanisms of cortical development, but also provide suggestions for ways to generate this cell type by direct reprograming from other cell linages.

\subsubsection{Mutation of IPC-enriched genes is implicated in human neurodevelopmental disorders and neuropsychiatric diseases}

The single-cell transcriptomic analysis of human developing cortex has identified a set of IPC genes (Fan et al., 2018; Li et al., 2018a; Nowakowski et al., 2017; Pollen et al., 2015; Zhong et al., 2018). As part of further investigations, we compared in silico expression of such human IPC genes with our identified mouse IPC transcriptome to identify the developmental and evolutionary origin of the transcriptional signature of IPC cells. Our data suggest the existence of both conserved and non-conserved transcriptional signatures of IPCs in mammalian evolution. Consistent with this line of evidence, previous studies have shown that expression of TBR2 was found specifically in IPCs in lissencephalic rodent brain (Englund et al., 2005). In gyrencephalic ferret or primates, TBR2 labeled IPCs, and almost half of SOX2+, PAX6+ 
bRGC population (Betizeau et al., 2013; Fietz et al., 2010; Florio and Huttner, 2014; Hansen et al., 2010; Turrero Garcia et al., 2016). Furthermore, in rodent cortex, IPCs are predominatelyneurogenic progenitors. However, in gyrencephalic species, IPCs are capable of selfamplification through symmetric proliferative divisions before their terminal division to generate neurons (Florio and Huttner, 2014; Lui et al., 2011). Beyond simply marking IPCs as transient progenitor cell type, future studies may have to relate and delve into the heterogeneity in the molecular milieu of IPCs in different species to afford elucidation of their contributions to cortical morphogenesis.

Advances in genetics and genomics studies in recent times have made it possible to identify many genetic coding and non-coding variants that cause neurodevelopmental disorders (D'Gama and Walsh, 2018; Hu et al., 2014; Juric-Sekhar and Hevner, 2019), with increased risk of neuropsychiatric disturbances (Sestan and State, 2018; Sullivan and Geschwind, 2019). Although we now have better insights into the genetic architecture of neuropsychiatric perturbations, we still lack a comprehensive description of the underlying molecular and cellular mechanisms, mainly because of the heterogeneity of risk loci, and the involvement of multiple cell types and brain regions. Therefore, knowledge of the regulatory networks and the spatiotemporal distribution of these networks in the brain, is essential for elucidating which cell types are relevant in the etiology and possible treatment of these neurodevelopment- and neuropsychiatry-related disorders. Moreover, the clarification of the mechanistic underpinnings of any given neurological disorder also requires detailed understanding of the developmental events that are disrupted in the course of the disease, non-genic causatives (environmental or epigenetic) of the anomalies, and dissection of the eventual phenotype.

Gene co-expression analyses have also revealed that the developing human (Kang et al., 2011; Miller et al., 2014; Pletikos et al., 2014) or mouse (Loo et al., 2019) brain transcriptome can be organized into distinct co-expression networks with often prominent spatiotemporal patterns, and enriched for distinct biological functions. By probing the transcriptome of mouse IPCs and performing further analysis in the form of phenotype association categorization, we found strong connection between the identified IPC genes and known human neurodevelopmental disorders (Fig. 2.5). This can be explained by the essential role of IPCs in cortical development. A great proportion of cortical neurons can be traced to IPCs. IPC-derived neurons predominately form the upper cortical layers and their axons constitute the large interhemispheric commissural system (i.e., the corpus callosum). Cortical expansion and evolutionary changes have been attributed to the tremendous neurogenic output of TBR2expressing IPCs and their diversity, especially in human. It is mainly for these reasons that disruption in the production of IPCs can lead to a wide range of cortical malformations and diverse neurological perturbations in the mammalian cortex. Our data thus suggest that diseaselinked mutations of IPC genes might form robust groupings based on their GO profiles. These diseases clearly link to neurodevelopmental defects, e.g. cortical size-associated disorders (microcephaly, macrocephaly, and abnormal cortical gyration), corpus callosum defects (dysplastic, agenesis, aplasia, hypoplasia of corpus callosum, and abnormality of the cerebral white matter), and neurological deficits (intellectual disability, psychomotor developmental delay, schizophrenia, autism, and epilepsy).

Despite the recent great interest in elucidating the principles underlying the IPCmediated evolutionary expansion of the neocortex and the consequence of related 
dysregulation, relatively less attention is accorded to dissecting disease-linked mutations of IPC genes to elucidate the pathophysiology of the attendant neurological disorder. By employing mouse model for the novel IPC gene Esco2, we were able to identify that IPCs may centrally rely on ESCO2 for survival and maintenance of their pool in the developing cortex. The absence of ESCO2, which is rather needed for the correct segregation of chromatids and therefore the genetic material into the progenies of dividing IPCs, may have triggered the massive apoptosis of the resultant ESCO2-deficient IPCs and the resultant overt cortical dysgenesis. Interestingly, Esco2 mutations in human have been linked to neurological phenotypes, including microcephaly and cognitive deficits. The said pivotal role played by ESCO2 in IPC genesis and cortical morphogenesis recapitulated similar critical function of TBR2 in brain morphogenesis. Mutations that abolish $T b r 2$ expression can cause severe neurodevelopmental abnormalities, including microcephaly, severe motor and cognitive delay, hypotonia, callosal agenesis, polymicrogyria, and cerebellar hypoplasia in rodent (Arnold et al., 2008; Sessa et al., 2008) and human (Baala et al., 2007). For future studies, linkage mapping and/or exome sequencing in human is expected to identify more IPC-related mutations and dysregulated genes associated with aberrant cortical architecture and growth.

To minimize gender bias in sampling for RNA-seq, we altogether used tissue from 15 embryos for 3 replicates. In addition, to examine the relative gender contribution in our samples, we compared the expression of chromosome X (Xist, Pgkl, Hprt, Eif2s3x) and chromosome Y (Ddx3y, Eif2s3y) - located genes, which are known housekeeping genes in the developing forebrain (Cheung et al., 2017; Dewing et al., 2003). The comparison revealed their comparable expression level in samples from TBR2+ IPCs and TBR2- cells (Fig. S2.1C). The data suggests that TBR2+ and TBR2- cell populations were derived from a similar number of female and male embryos. Nevertheless, the expression pattern of chromosome X/Y-located IPC genes should be validated by either IHC or ISH afore further investigation. 


\section{Chapter 3: Post-transcriptional regulation by the exosome complex is required for cell survival and forebrain development via repression of P53 signaling}

All elements in this chapter have already been published in:

Ulmke, P.A., Xie, Y., Sokpor, G., Pham, L., Shomroni, O., Berulava, T., Rosenbusch, J., Basu, U., Fischer, A., Nguyen, H.P., Staiger, J.F., Tuoc T. (2021). Post-transcriptional regulation by the exosome complex is required for cell survival and forebrain development via repression of P53 signaling. Development 148(3), dev188276.

Personal contributions: I was involved in conducting the experiments, data analyses, and writing of the manuscript.

\subsection{Abstract}

Fine-tuned gene expression is crucial for neurodevelopment. The gene expression program is tightly controlled at different levels, including RNA decay. $\mathrm{N}^{6}$-methyladenosine (m6A) methylation-mediated degradation of RNA is essential for brain development. However, m6A methylation impacts not only RNA stability, but also other RNA metabolism processes. How RNA decay contributes to brain development is largely unknown. Here, we show that EXOSC10, a RNA exonuclease subunit of the RNA exosome complex, is indispensable for forebrain development. We report that cortical cells undergo overt apoptosis, culminating in cortical agenesis upon conditional deletion of EXOSC10 in mouse cortex. Mechanistically, EXOSC10 directly binds and degrades transcripts of the P53 signaling-related genes, such as AEN and BBC3. Overall, our findings suggest a crucial role for EXOSC10 in suppressing the P53 pathway, in which the rapid turnover of the apoptosis effectors AEN and BBC3 mRNAs is essential for cell survival and normal cortical histogenesis.

\subsection{Introduction}

The neocortex of the mammalian brain is radially structured into six neuronal layers and multiple functional domains that form the structural basis for sensorimotor processing and intellectual ability. In early cortical development, apical progenitors (APs) in the ventricular zone (VZ) function as neural stem cells (NSCs) and produce neurons via direct and indirect neurogenesis in a specific temporal order, which will make up the different cortical layers (Gotz and Huttner, 2005; Kriegstein et al., 2006). In direct neurogenesis, APs divide asymmetrically to generate new Aps and neurons. Neurons produced during early neurogenesis are distributed mainly in the lower cortical layers (LLs) L6 and L5. In indirect neurogenesis, APs divide to self-renew and produce basal progenitors (BPs) that undergo limited cycles of symmetric divisions to generate more neurons with upper layers (ULs) L4-L2 (Pontious et al., 2008).

Optimal regulation of gene expression is crucial for establishing the intricate balance between the rate of proliferation and differentiation of neural progenitor cells as well as cell viability and apoptosis. Transcriptional regulation plays a central role in controlling gene expression. However, regulation of gene expression is not limited to the transcriptional level. 
Posttranscriptional mechanisms, such as the regulation of RNA stability contribute in sharpening the expression of genes during development.

The evolutionarily conserved RNA exosome is an essential factor that modulates gene expression during development (Januszyk and Lima, 2014; Kilchert et al., 2016). The ring-like structured exosome complex contains 11 different exosome component (EXOSC) subunits, including nine structural subunits (EXOSC1-9) and two catalytic subunits (EXOSC10, DIS3) (Januszyk and Lima, 2014; Kilchert et al., 2016). Together with structural counterparts, EXOSC10 and DIS3 are able to degrade numerous RNAs using their ribonuclease activity. This makes the entire exosome complex indispensable for controlling the richness of RNAs, degrading malfunctional or mis-configured RNAs. The integrated EXOSC subunits, which interact through composite surfaces with their co-factors, are essential for targeting the exosome to specific RNAs for degradation, therefore conferring functional specificity (Januszyk and Lima, 2014; Kilchert et al., 2016; Lim et al., 2017; Lubas et al., 2011; Puno and Lima, 2018; Schmid and Jensen, 2019).

Of note, mutations of the EXOSC genes have been found in various human brain disorders, including corpus callosum hypoplasia, cerebellar atrophy, abnormal myelination, pontocerebellar hypoplasia with cerebellar and spinal motor neuron degeneration, and intellectual disability (Boczonadi et al., 2014; Burns et al., 2018; Di Donato et al., 2016; Fasken et al., 2020; Morton et al., 2018; Wan et al., 2012), suggesting important roles for the exosome complex in neural development.

To investigate the possible involvement of the exosome complex in brain development, we used a conditional knockout (cKO) of the RNA exonuclease subunit EXOSC10 from early brain development in transgenic mice. Transcriptional profiling of the EXOSC10cKO cortical tissue revealed that EXOSC10 suppresses the expression of large sets of genes involved in various processes of brain development, including cell death-related pathways. RIP-seq and RNA degradation analyses uncovered that EXOSC10 directly binds to and induces degradation of P53 signaling-related transcripts. Phenotypically, we found that elimination of EXOSC10 leads to a massively enhanced apoptosis, reduced neurogenesis and dysgenesis of cortical layers, with the first effect being rescued by inhibition of P53 signaling. Overall, this study provides new insights into the post-transcriptional regulatory mechanism mediated by the RNA exosome complex, which acts upstream of P53 signaling in apoptosis suppression in brain development.

\subsection{Results}

\subsubsection{Expression of EXOSC10 is indispensable for forebrain development and formation of cortical layers}

Mutations in human EXOSC2, EXOSC3, EXOSC8 and EXOSC9 genes, and their associated brain disorders imply important roles for the exosome complex in neurodevelopment (Boczonadi et al., 2014; Di Donato et al., 2016; Wan et al., 2012). In further support of the involvement of the exosome complex in mammalian brain development, exosome genes are prominently expressed in the developing mouse cortex, especially in the ventricular zone (VZ) (Fig. 3.1A; Fig. S3.1A-J). Extracting the published scRNA-seq dataset of the mouse developing 
cortex (Telley et al., 2016) also confirmed the highest expression of exosome genes in apical progenitors (APs), which are found in the VZ (Fig. 3.1B; Fig. S3.1K-S). As EXOSC10 is the exonuclease subunit of the RNA exosome complex (Fig. S3.1A), we aimed to study the role of EXOSC10 in neural development by generating and characterizing the cortical phenotype of EXOSC10 conditional knockout (cKO) mice.

To investigate the consequences of loss of EXOSC10 in brain development, we bred mice bearing conditional inversion (COIN) alleles of EXOSC10 (Economides et al., 2013; Pefanis et al., 2015) with different Cre lines, including telencephalon-specific FoxG1-Cre (Hebert and McConnell, 2000) and cortex-specific Emxl-Cre (Gorski et al., 2002), to generate the corresponding cKO mutants: cKO_FoxG1-Cre and cKO_Emxl-Cre. Telencephalonspecific cKO_FoxG1-Cre embryos exhibited an absolute absence of the telencephalon at E17.5 (Fig. S3.2A, arrow). To examine formation of the telencephalon at early stages, immunohistochemistry analysis was performed with antibodies against SOX2, PAX6, HUCD and NEUN in forebrain tissue of control and cKO_FoxG1-Cre embryos between E10.5 and E12.5 (Fig. S3.2B,C). The expression of these markers was found in telencephalon (Tel), diencephalon (Di) and mesencephalon (Mes) of control embryos, whereas their expression was seen only in Di and Mes structures in cKO_FoxG1-Cre mutants. This finding suggests that the deletion of EXOSC10 at the onset of telencephalon formation in cKO_FoxG1-Cre embryos results in the failure of telencephalon formation, which makes this mouse line inappropriate for further investigations. At P6, the cortex in $\mathrm{CKO}_{-} E m x \mathrm{I}$-Cre mice was significantly smaller than that of the control mice; however, we were still able to examine cortical development under EXOSC10 deficiency (Fig. 3.1C,D).

The cKO mutants at P6 had visually smaller cortical size and thinner cortical layers than that of controls, as revealed by SATB2 immunostaining (Fig. 3.2A,G). To explore cortical layer formation in detail, we performed immunohistochemistry and evaluated the expression of cortical layer (L)-specific markers, such as REELIN (L1; Fig. 3.2B), CUX1 (L2/3; Fig. 3.2C), CTIP2 (L5; Fig. 3.2D), SOX5 (L5/6; Fig. 3.2E) and TBR1 (L6; Fig. 3.2F).

The population of REELIN+ L1 neurons generated in the cortical hem and ventral telencephalon, where the Emxl promoter is not active (Gorski et al., 2002), seems to be preserved in the cortex of cKO_Emxl-Cre mutants (Fig. 3.2B,G). In contrast, the numbers of CUX1+ L2/3, CTIP2+ L5, SOX5+ L5/6 and TBR1+ L6 neurons, which are generated from cortical progenitors, were significantly reduced in the cKO_Emxl-Cre cortex (Fig. 3.2C-G). Together, these results show that the expression of EXOSC10 is required for normal forebrain development and cortical layer formation. 
A
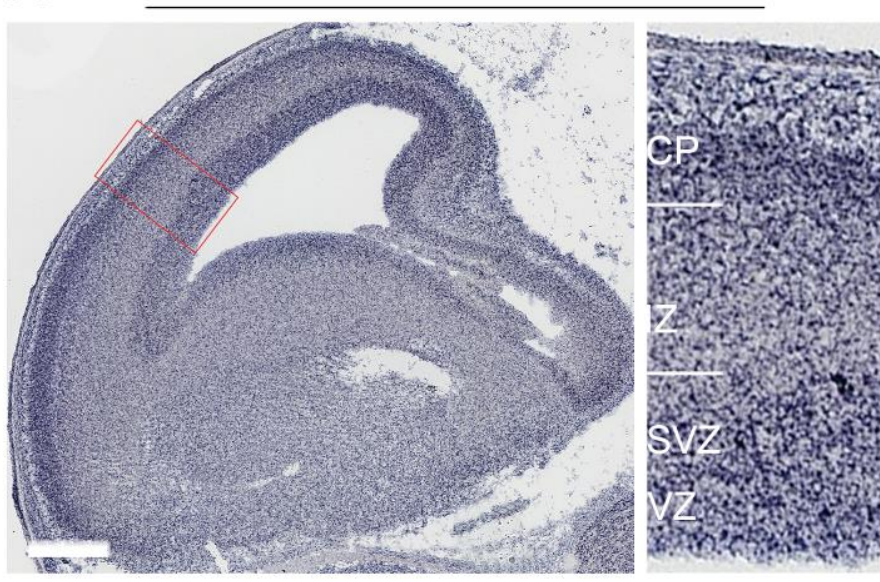

C Early cortical progenitor-specific Emx1-Cre
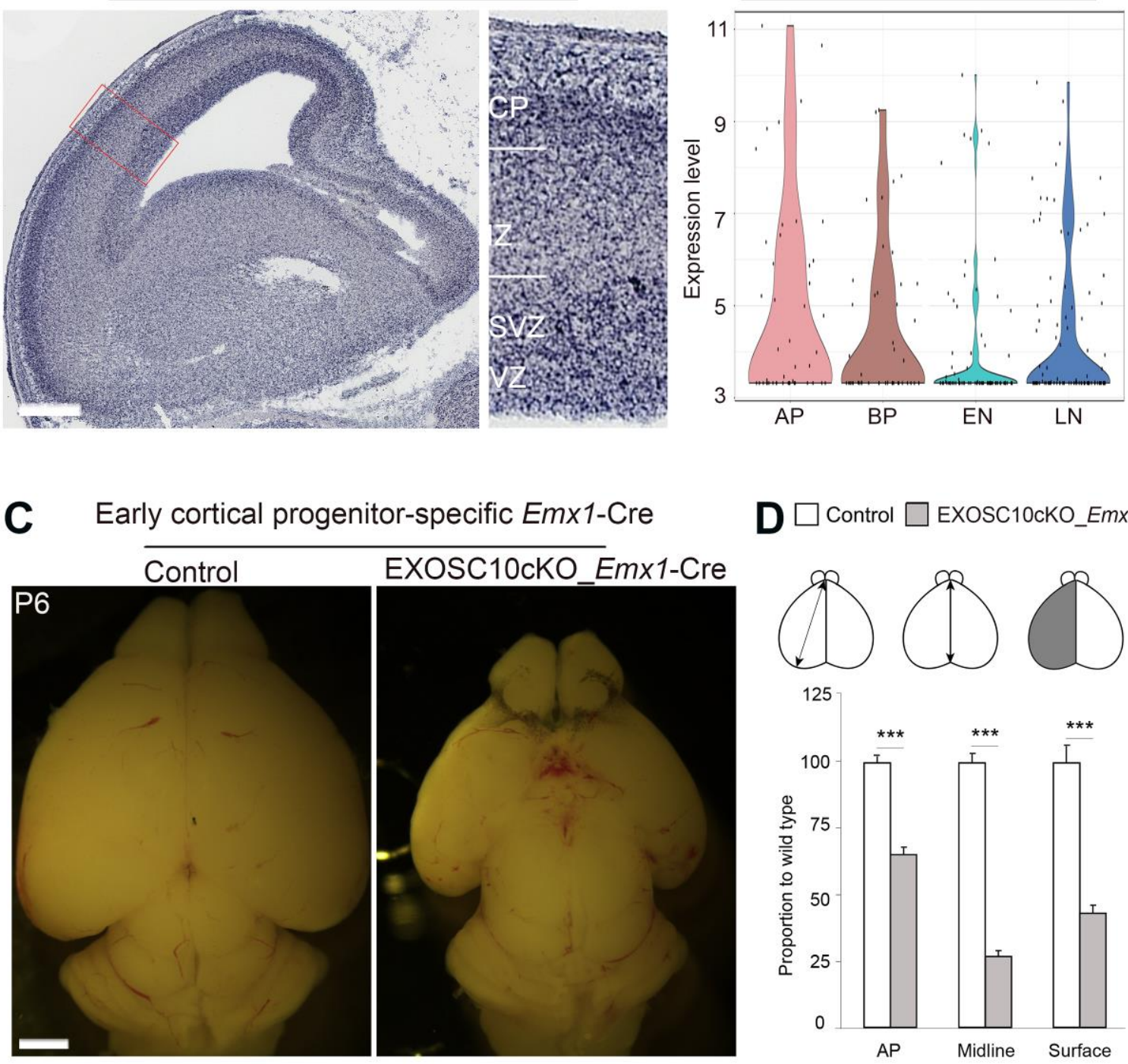

D $\square$ Control $\square$ EXOSC10cKO_Emx1
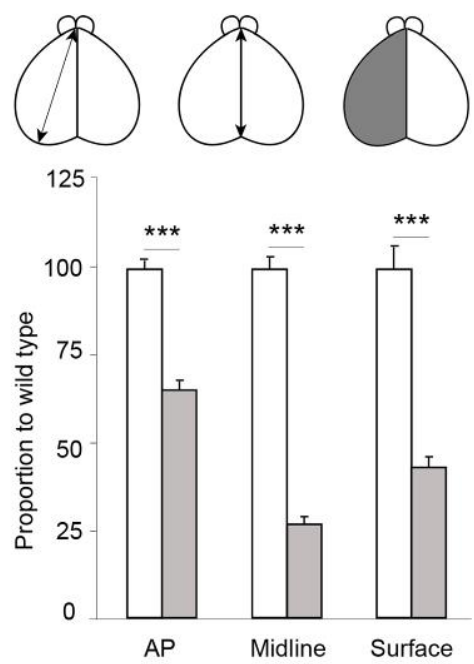

Figure 3.1 The expression of EXOSC10 is indispensable for cortical development.

(A,B) In situ hybridization analysis of sagittal sections obtained from GenePaint database (Visel et al., 2004) (A) and in scRNA-seq analysis (Telley et al., 2016) (B) of the E14.5 mouse cortex reveal that EXOSC10 is widely expressed, with its highest level seen in apical progenitors (AP, in B) in the ventricular zone (VZ, in A). (C) At P6, the cortex in cKO_Emxl-Cre mice is distinctly smaller than that of controls. (D) Quantification of the cortical proportions in cKO_Emxl-Cre mice relative to control showing significant differences. AP, apical progenitors/RGCs; BP, daughter basal progenitors/IPCs; EN, early-born neurons; LN, late-born neurons; CP, cortical plate; SVZ, subventricular zone; VZ, ventricular zone. $* * * P<0.005$. Scale bars: $1 \mathrm{~mm}$. 

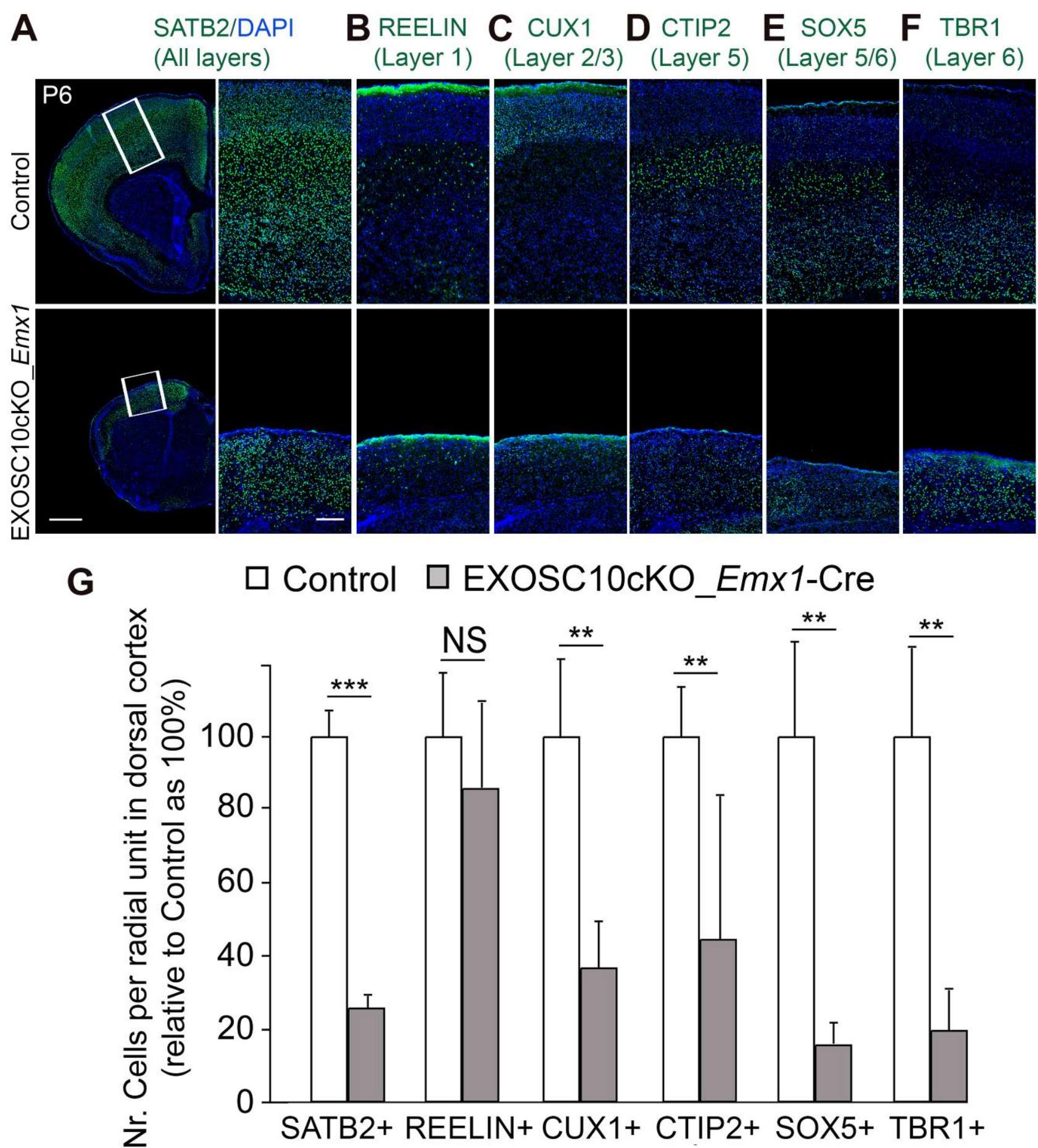

Figure 3.2 Loss of neurons and a reduction in the thickness of cortical layers in postnatal EXOSC10cKO mutants.

(A-F) Immunohistochemistry showing cortical expression of neuronal markers specific for certain cortical layers: SATB2 (all layers, A), REELIN (layer 1, B), CUX1 (layer 2/3, C), CTIP2 (layer 5, D), SOX5 (layer 5/6, E) and TBR1 (layer 6, F) in coronal sections of EXOSC10cKO and control at P6. Right images are higher magnifications of fields indicated by white frames. (G) Quantification of the neuronal markers in EXOSC10cKO cortex at P6 relative to control. The cells were counted in the dorsal area at the rostral levels shown in A. $* * P<0.01$, $* * * P<0.005$; NS, not significant. Scale bars: $1 \mathrm{~mm}$ and $200 \mu \mathrm{m}$ (higher magnification). 


\subsubsection{EXOSC10 ablation in early cortical development causes massive apoptosis}

The diminished population of neurons in the cKO cortex at postnatal stage promoted us to investigate the consequences of the loss of EXOSC10 expression on neurogenesis at early embryonic stages. Consistent with the decreased number of neurons in cortical layers at P6 (Fig. 3.2), the EXOSC10-ablated cortex at E13.5 displayed a diminished number of HUCD+ and NEUN+ neurons in cortical plate (CP) (Fig. 3.3A,B,D,E). Remarkably, the thickness of the germinal zones, i.e. the ventricular zone (VZ) and sub-ventricular zone (SVZ) (Fig. 3.3A-C), in the control cortex was comparable with that of the mutant cortex (Fig. 3.3A,B). Accordingly, the number of apical progenitors (PAX6+ or SOX2+) in VZ and basal progenitors (TBR2+) in $\mathrm{SVZ}$ was comparable in $\mathrm{cKO}$ and control cortices (Fig. 3.3A-E). These findings suggest that the deletion of EXOSC10 might lead to an enhanced apoptosis or defect in neuronal differentiation in early cortical development, causing the observed brain microcephaly at the postnatal stage.

The immunohistochemical analysis with the apoptosis marker activated caspase 3 (CASP3) revealed that EXOSC10 ablation engenders intense apoptosis in EXOSC10cKO_Emxl-Cre cortex at E13.5, especially in the rostromedial area (Fig. 3.4A-C), where the Cre recombinase activity was found to be highest (Gorski et al., 2002; Narayanan et al., 2015; Nguyen et al., 2018). A dramatic increase in apoptosis was already evident in the mutant cortex at E11.5 (Fig. 3.4D). Apoptotic cells were observed in the entire mutant cortex, albeit more dominant in the basal side of cortical wall, suggesting that neurons were the most affected population of cells therein (Fig. 3.4A-C). Notably, there was no difference in the number of CASP3+ apoptotic cells between control and EXOSC10cKO cortices at postnatal stages, indicating that EXOSC10 expression is required for cell viability only at embryonic stages of cortical development (Fig. S3.3).

Double immunohistochemical analyses of CASP3 and markers for apical progenitors (SOX2; Fig. 3.4A), basal progenitors (TBR2; Fig. 3.4B) and neurons (NEUN; Fig. 3.4C) confirmed that apoptosis was found in all three cell populations in the cKO_Emxl-Cre cortex (indicated by filled arrows in Fig. 3.4A-C, E). Notably, the highest cell death rate was identified in NEUN+ neurons (Fig. 3.4E). Altogether, our findings indicate that EXOSC10 is crucial for cell viability in early cortical development. 
A

Rostral level

Caudal level
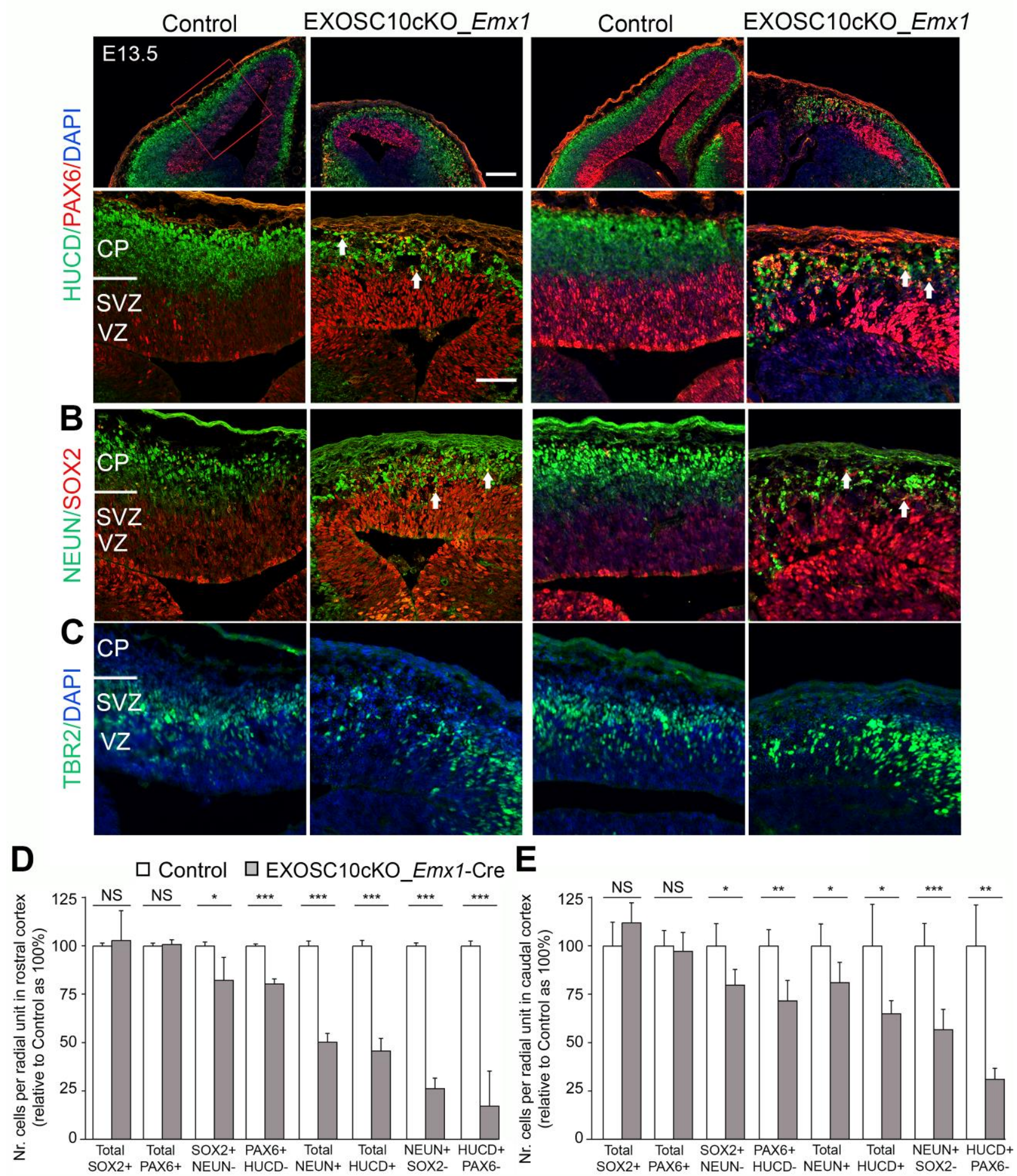

Figure 3.3 A reduced thickness of the cortical plate but normal pools of cortical progenitors in EXOSC10cKO cortex at E13.5.

(A,B) Double immunohistochemical analyses with antibodies that specifically mark NSCs [PAX6 (A) and SOX2 (B)] and neurons [HUCD (A) and NEUN (B)] in the EXOSC10cKO cortex and control at E13.5. For each, rostral and caudal sections are shown. Lower images are higher magnifications of fields indicated by the red frame. In cKO cortex, many PAX6+ and SOX2+ cells were also immunoreactive to HUCD and NEUN (A and B, white arrows). (C) Immunohistochemistry showing cortical expression of the intermediate progenitor marker TBR2 in the E13.5 EXOSC10cKO and control cortex. (D,E) Quantification of cells expressing neuronal progenitor genes (SOX2 and PAX6) and neuronal genes (NEUN and HUCD) in the E13.5 cKO cortex relative to control at rostral (D) and caudal (E) levels. CP, cortical plate; SVZ, subventricular zone; VZ, ventricular zone. $* P<0.05, * * P<0.01$, $* * * P<0.005$; NS, not significant. Scale bars: $200 \mu \mathrm{m}$ and $100 \mu \mathrm{m}$ (higher magnification). 
A

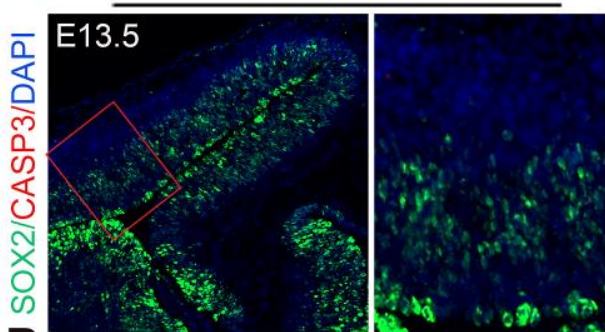

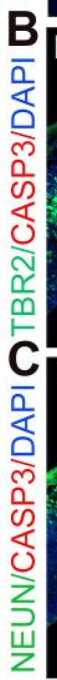

E13.5
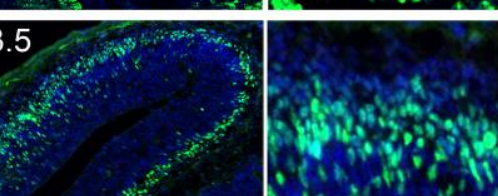

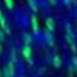

1,10

13
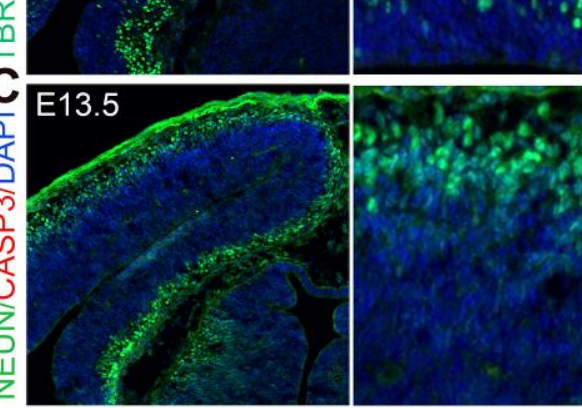

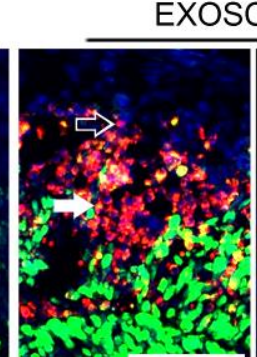

EXOSC10cKO_Emx1
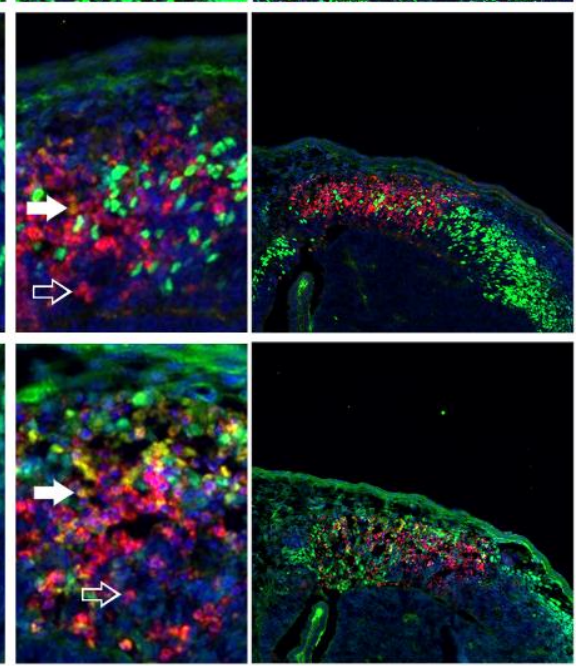

D

$\square$ Control $\square$ EXOSC10cKO_Emx1-Cre

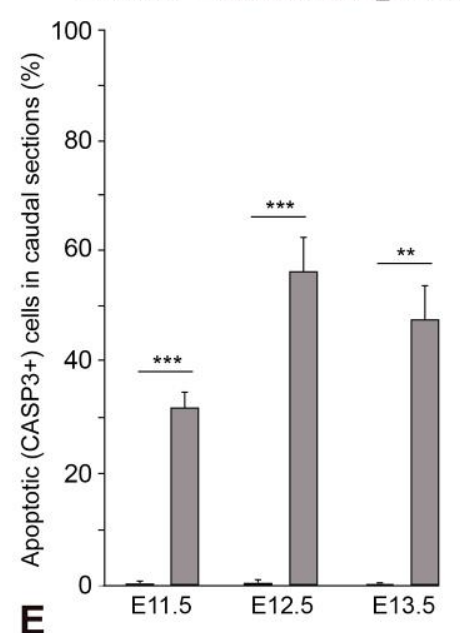

$\mathrm{E}$

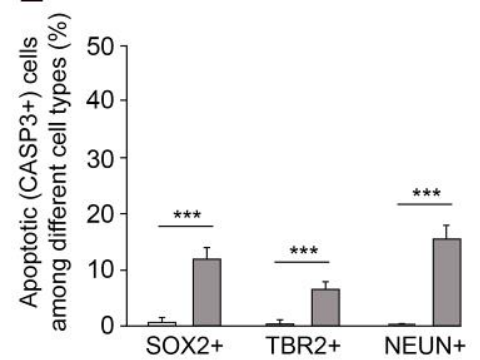

Figure 3.4 EXOSC10 ablation in early cortical progenitors causes massive apoptosis.

(A-C) Double immunohistochemical analyses for the apoptosis marker activated caspase 3 (CASP3) together with SOX2 for NSCs (A), TBR2 for IPs (B) or NEUN for neurons (C) in the EXOSC10cKO cortex and control at E13.5. Middle images are higher magnifications of fields indicated by the red frame. White arrows indicate cells co-expressing CASP3 and the cell type marker; empty arrows indicate cells expressing only CASP3. (D) Quantification of apoptotic cells in EXOSC10cKO cortex and control at E11.5, E12.5 and E13.5. (E) Quantification of apoptosis in different cell types by counting CASP3+ cells among SOX2-, TBR2- or NEUNexpressing cells in the E13.5 cKO and control cortex. CP, cortical plate; SVZ, subventricular zone; VZ, ventricular zone. $* * P<0.01, * * * P<0.005$; NS, not significant. Scale bars: $200 \mu \mathrm{m}$ and $100 \mu \mathrm{m}$ (higher magnification).

\subsubsection{Identification of EXOSC10 target genes}

To understand the molecular mechanisms underlying control of cortical development by EXOSC10 and to identify the EXOSC10 direct target transcripts, RNA sequencing (RNA-seq) as well as RNA immunoprecipitation sequencing (RIP-seq), were performed. In the RNA-seq, RNAs isolated from the E12.5 EXOSC10cKO_Emxl-Cre and control cortices were sequenced (Fig. S3.4A,C). We found that loss of EXOSC10 caused upregulation of 1031 genes and downregulation of 844 genes (adjusted $\mathrm{P}<0.05$; Fig. 3.5A, Table $\mathrm{S} 1$ ). Gene ontology (GO) analysis of the upregulated genes reflected involvement of various brain development processes (Fig. 3.5B, Table S2). Consistent with the increased apoptosis, cell death-related pathways, such as regulation of apoptotic signaling pathway, and signal transduction by P53 mediators were upregulated in the cKO cortex (Fig. 3.5C, Table S2).

In our RIP-seq experiment, RNAs were purified from the E12.5 wild-type mouse cortex (Fig. S3.4B,D). By sequencing the EXOSC10-bound RNAs, binding enrichment of EXOSC10 on 3159 transcripts was identified (adjusted $\mathrm{P}<0.05$; Fig. 3.5B, Table S3). GO analysis revealed that the EXOSC10-bound transcripts participate in various processes of brain development (Fig. 3.5D, Table S4). To identify candidates for functional analysis, we compared the RNAs upregulated in the EXOSC10cKO cortex in RNA-seq experiment with transcripts bound by 
EXOSC10 in RIP-seq analysis. We identified 144 transcripts common to the results of both experimental analyses (Fig. 3.5E, Table S5). Interestingly, those intersectional 144 upregulated genes showed an enrichment in the GO term 'neuron death' (Fig. 3.5F, Table S6).

In accordance with our immunohistochemical data showing increased numbers of CASP3+ apoptotic cells, many genes involved in P53 apoptosis signaling (e.g. Ccng1, Sesn2, Pmaip1, Bbc3 and Aen) were upregulated in the cortex of EXOSC10cKO mutants (Fig. 3.5A; Fig. 3.6B). Furthermore, the results from our RIP-seq analysis revealed that transcripts of many apoptosis-related genes (e.g. Ccne1, Ccng2, Tsc2, Bbc3, Apaf1 and Aen) were bound by EXOSC10 (Fig. 3.5B; Fig. 3.6A). Thus, our findings raise the possibility that EXOSC10 inhibits apoptosis by directly suppressing expression of P53 signaling effector genes.

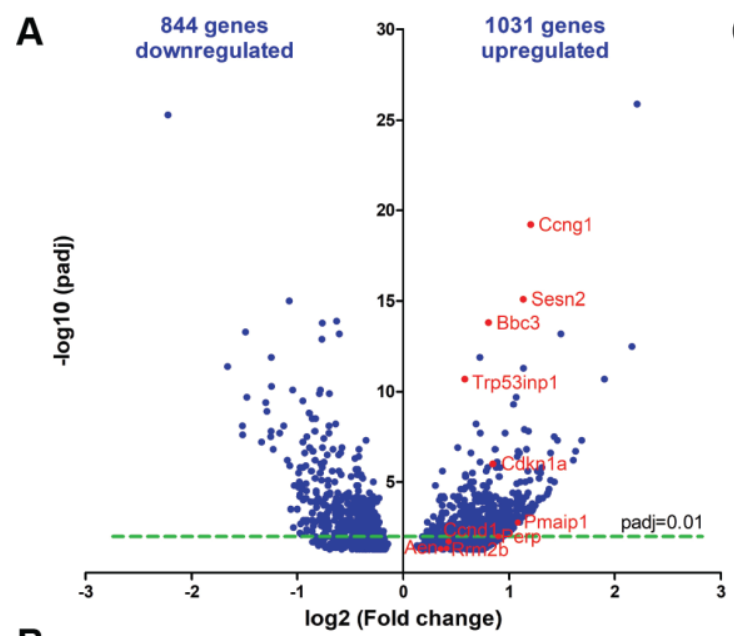

B

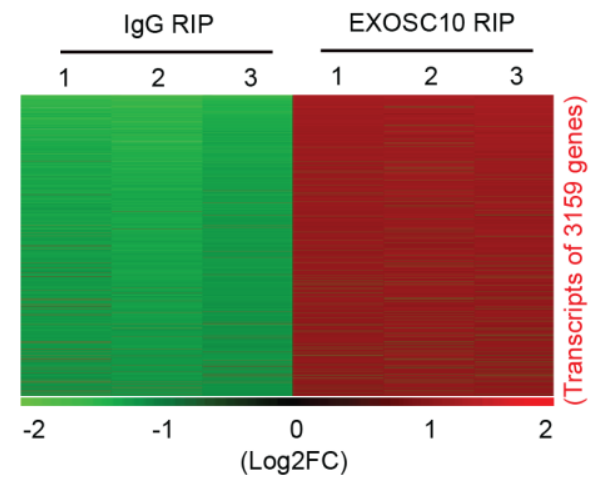

E

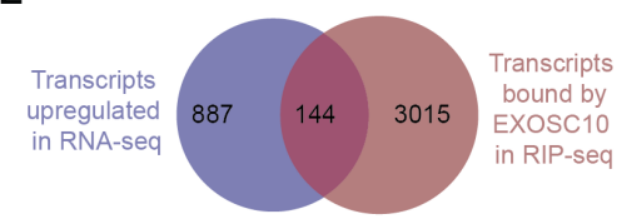

$\mathbf{F}$

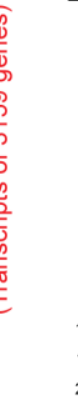

C

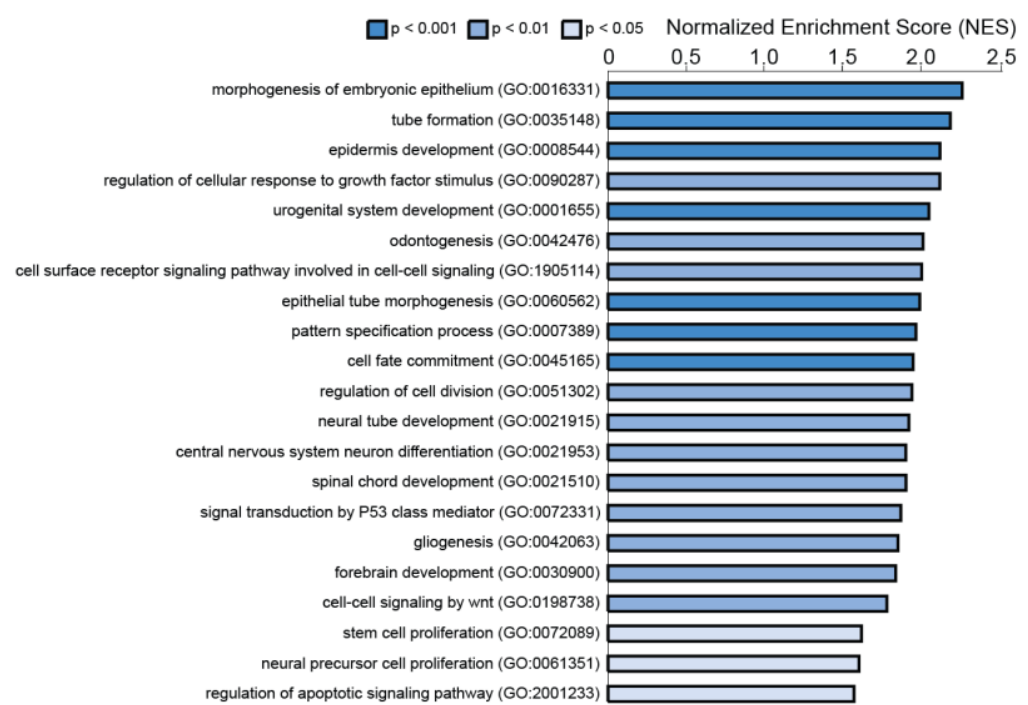

D

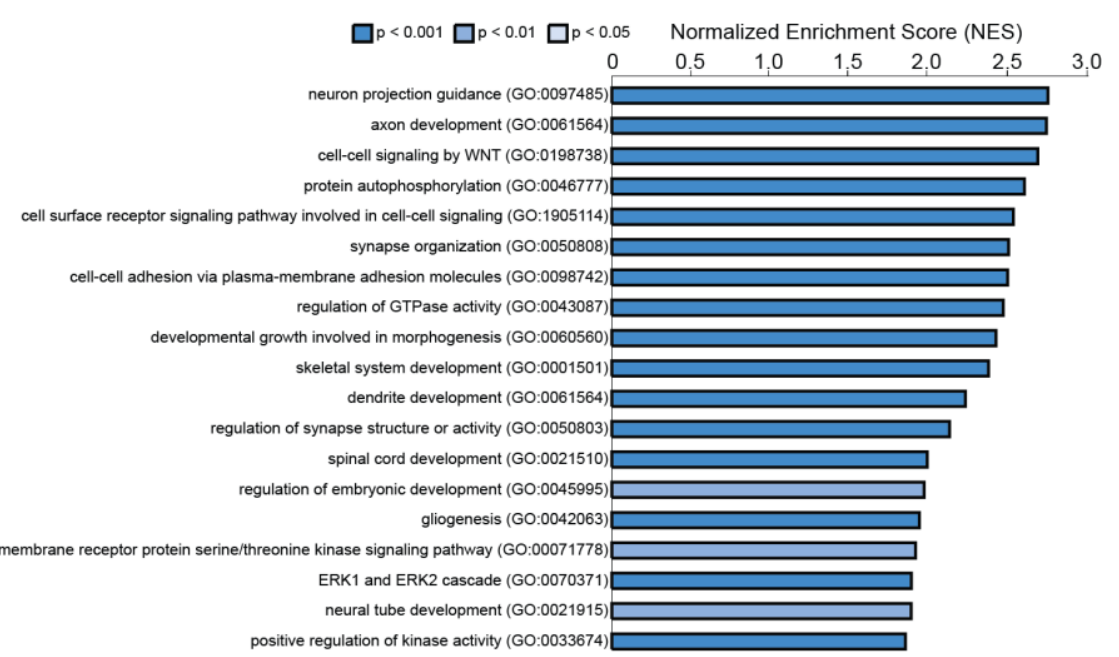

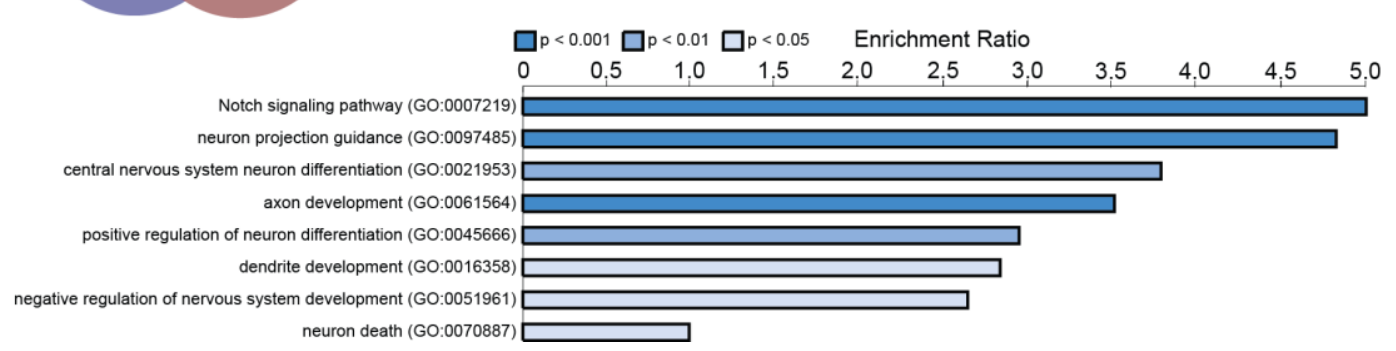


Figure 3.5 EXOSC10 controls global gene expression in early cortical development.

$(\mathrm{A}, \mathrm{B})$ Volcano plot and heatmap showing significant changes in gene expression (A) and transcript-binding enrichment (B) revealed by RNA-seq (E12.5 EXOSC10cKO cortex versus control) and RIP-seq (E12.5 cortex, EXOSC10 antibody versus IgG) analyses, respectively. (C,D) Gene ontology analysis of genes upregulated in RNA-seq (C) and transcript-binding enrichment in EXOSC10 RIP-seq (D). (E) The overlap between upregulated genes in RNA-seq of E12.5 EXOSC10cKO cortices and transcript-binding enrichment in EXOSC10 RIP-seq. (F) Gene ontology analysis of upregulated genes in RNA-seq and their transcript bound by EXOSC10 in RIP-seq.
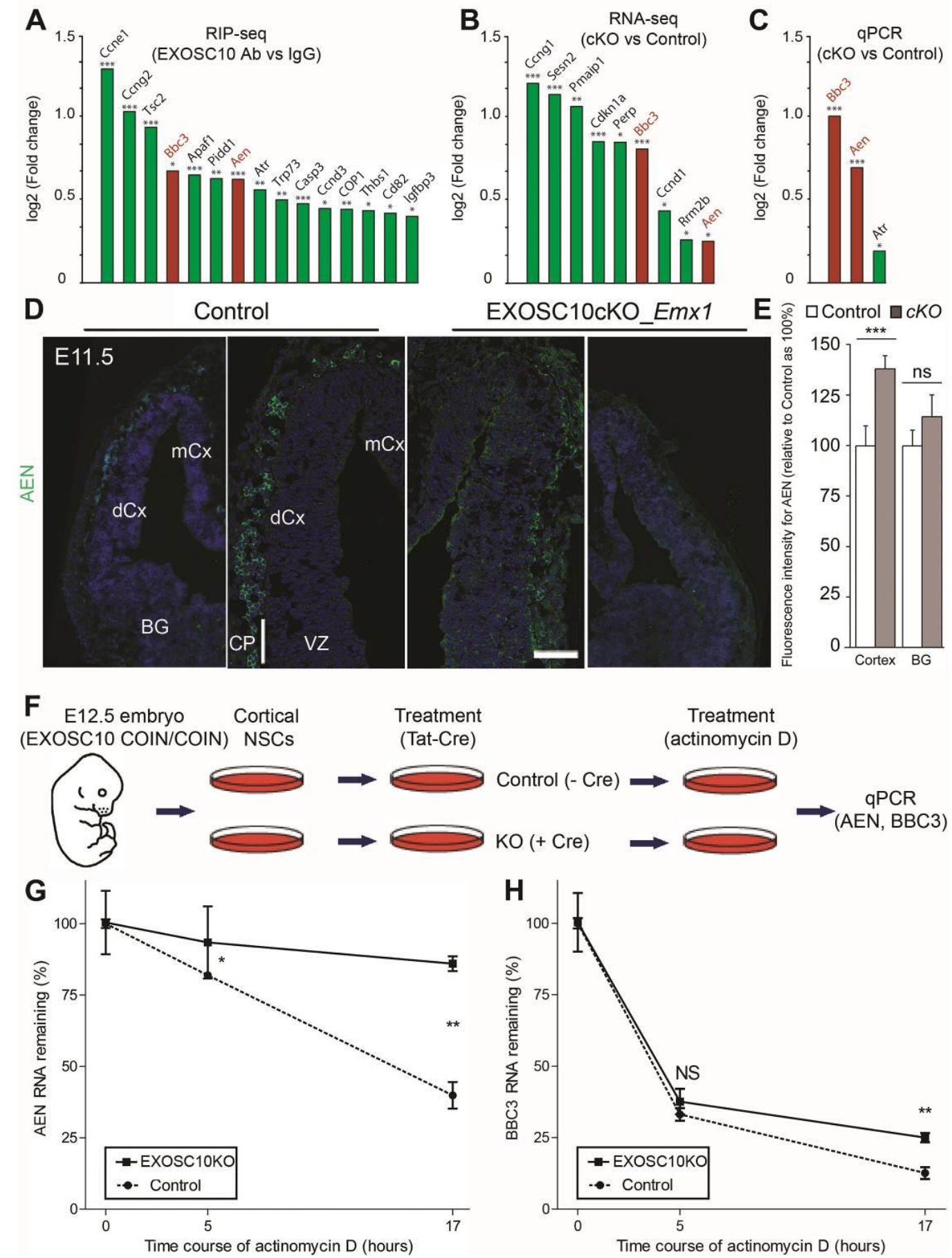

Figure 3.6 EXOSC10 suppresses the expression of P53 pathway AEN and BBC3 genes by degrading their transcripts.

(A) RIP-seq analysis with RNA from E12.5 mouse cortex revealed the binding of EXOSC10 to P53 pathway transcripts. (B,C) The upregulated genes in EXOSC10cKO_Emxl-Cre cortex at E12.5, which are involved in P53 signaling, were identified by RNA-seq (B) and confirmed by qPCR (C). (D,E) Immunohistochemistry (D) and quantitative analyses (E) for expression of AEN on coronal sections of control and EXOSC10cKO cortices at E11.5 are shown. (F) Experimental paradigm for the RNA degradation assay using cultured cortical NSCs. (G,H) RNA degradation assay showing changes in the RNA abundance of AEN $(\mathrm{G})$ and BBC3 $(\mathrm{H})$ measured by qPCR from EXOSC10 KO NSCs and control NSCs after actinomycin D treatment for $0 \mathrm{~h}, 5 \mathrm{~h}$ and $17 \mathrm{~h}$. BG, basal 
ganglia; $\mathrm{CP}$, cortical plate; $\mathrm{dCx}$ and $\mathrm{mCx}$, dorsal and medial cortex; $\mathrm{VZ}$, ventricular zone. ${ }^{*} P<0.05,{ }^{*} * P<0.01$, $* * * P<0.005$; NS, not significant. Scale bar: $100 \mu \mathrm{m}$.

\subsubsection{P53 pathway genes Aen and Bbc3 are direct targets of EXOSC10 in the developing cortex}

Among the transcripts belonging to the P53 pathway, AEN (apoptosis enhancing nuclease) and BBC3 (BCL2 binding component 3; also known as PUMA, P53-upregulated modulator of apoptosis) were bound by EXOSC10 (Fig. 3.6A). Their upregulated expression in the EXOSC10cKO cortex was first revealed by RNAseq (Fig. 3.6B), then confirmed by qPCR (for BBC3, AEN and Atr; Fig. 3.6C) and immunohistochemical analyses (for AEN; Fig. 3.6D,E), making them strong candidates for mediating regulation of apoptosis by EXOSC10.

To ascertain the functional effect of EXOSC10 binding, we examined whether EXOSC10 deletion influences decay of these identified transcripts. For this purpose, an RNA degradation assay was performed using cultured cortical NSCs derived from E12.5 EXOSC10 COIN/COIN embryos. NSCs were treated with either soluble Tat-Cre recombinase to knockout EXOSC10 (EXOSC10KO) or vehicle as a control group (Fig. 3.6F; Fig. S3.5). An actinomycin D-based method was used to halt de novo transcription (Yoon et al., 2017). The cells were harvested and qPCR was performed to quantify the transcript level of AEN and BBC3 in cultured NSCs before $(0 \mathrm{~h})$ and after $5 \mathrm{~h}$ and $17 \mathrm{~h}$ treatment of actinomycin D (Fig. 3.6F). Compared with control, a higher stability of AEN transcripts in EXOSC10KO NSCs was observed after $5 \mathrm{~h}$ and $17 \mathrm{~h}$ treatment of actinomycin D (Fig. 3.6G). The higher RNA stability of BBC3 in mutant NSCs than that in controls was seen after $17 \mathrm{~h}$ of actinomycin D treatment (Fig. 3.6H). These results indicated that EXOSC10 directly binds and degrades transcripts of AEN and BBC3.

To consolidate our observation that EXOSC10 may regulate apoptosis via suppression of the P53 apoptosis pathway, in vivo rescue experiments were performed. We used a P53 inhibitor, pifithrin- $\alpha$ (PFT $\alpha$ ), that is known to inhibit P53-dependent activation of P53-targeted genes (Komarov et al., 1999). PFT $\alpha$ was injected daily starting from 9.5 days post coitum (d.p.c.), and the PFT-treated EXOSC10cKO animals were examined at E13.5 (Fig. 3.7A). Whereas $52.0 \pm 3.5 \%$ of cells in the PFT-untreated (noninjected) EXOSC10cKO cortex were apoptotic, the percentage of CASP3+ cells decreased to $31.0 \pm 9.2 \%$ upon PFT $\alpha$ treatment (Fig. 3.7B,C). Thus, the observed apoptotic phenotype in EXOSC10cKO mutants was largely rescued by inhibition of the P53 pathway in the developing cortex. Among the P53 pathwayrelated genes, we compared the expression of $B b c 3$ and Aen from control and cKO cortices, which were treated with either vehicle or P53 inhibitor. Remarkably, PFT $\alpha$ treatment does not significantly rescue the aberrant upregulation of AEN upon the loss of EXOSC10 in the developing cortex (Fig. 3.7D). Treatment with PFT $\alpha$, however, decreases the expression of $\mathrm{BBC} 3$, which is upregulated in cKO cortex (Fig. 3.7E). This is in line with the evidence that BBC3 (but not AEN) is a direct target of P53, as the promoter region of BBC3 contains P53binding sites and can be directly activated by P53 (Han et al., 2001).

To address whether EXOSC10 regulates cortical development partly via suppression of P53 signaling, pregnant mice between 9.5 and 15.5 days post coitum (d.p.c.) were intraperitoneally injected daily with PFT $\alpha$ solution. Owing to the perinatal lethality of PFT $\alpha$ treated animals, the brain samples were collected at E18.5 for phenotype analysis (Fig. 3.8A). 
The expression of SATB2, which marks the majority of projection neurons in all cortical layers and areas (Alcamo et al., 2008; Britanova et al., 2008), was then examined in control, and in cKO with and without PFT $\alpha$ treatment. The treatment with P53 inhibitor did not influence the size of wild-type (control) cortex, as indicated by SATB2 expression. Remarkably, when compared with vehicle-treated cKO embryos, PFT $\alpha$-treated embryos had a significantly larger cortex. Concurrently, PFT $\alpha$ administration in cKO mutants resulted in an increase in the number of SATB2+ neurons (Fig. 3.8B-F). The findings indicate that treatment with the P53 inhibitor partly rescues the aberrant cortical morphology in mutants.

Altogether, these results reveal that loss of EXOSC10 during early cortical development causes aberrantly enhanced expression of P53 pathway-related transcripts such as AEN and BBC3, and causes increased apoptosis similar to that observed after P53 overexpression (Fig. 3.8G). These findings demonstrate that the balance between the RNA exosome complex and P53 signaling activity is essential for cell survival and for normal cortical development (Fig. $3.8 \mathrm{G})$.
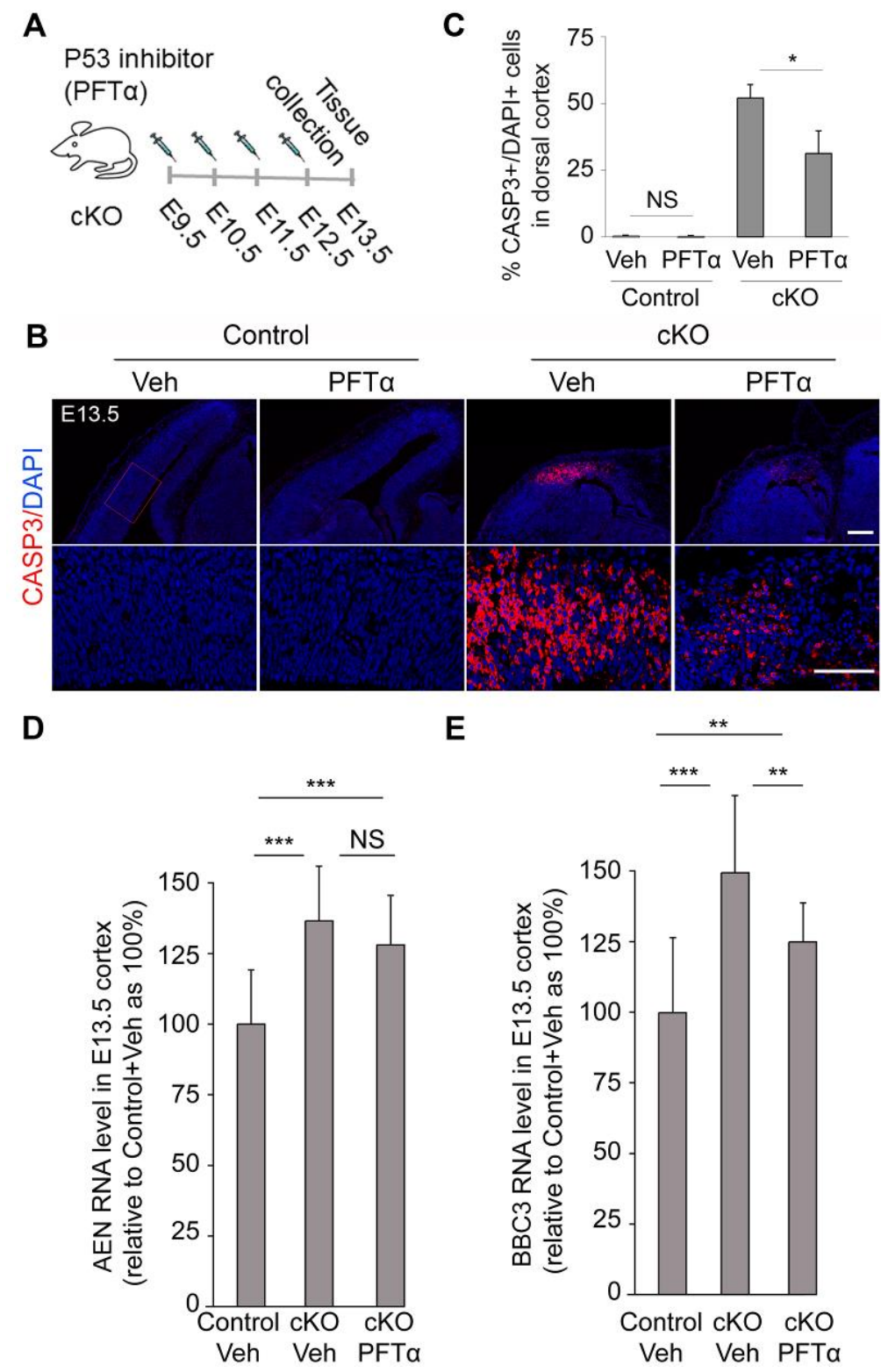

Figure 3.7 EXOSC10 suppresses apoptosis by inhibiting activity of P53 signaling pathway.

(A) Rescue experimental paradigm with the P53 inhibitor (PFT $\alpha$ ). (B,C) Immunohistochemistry (B) and quantitative analyses (C) for the apoptosis marker CASP3 on coronal sections of E13.5 control and 
EXOSC10cKOcortices with or without PFT $\alpha$ treatment. Lower images are higher magnifications of field indicated by the red frame (B). The ratio of CASP3+ cells to all DAPI+ cells was assessed in control and EXOSC10cKO cortices with or without PFT $\alpha$ treatment, showing a reduction in CASP3+ cells in the cKO upon injection (C). $(\mathrm{D}, \mathrm{E})$ Treatment with PFT $\alpha$ decreases the expression of BBC3 (E), but not that of AEN (D), which is upregulated in cKO cortex. ${ }^{*} P<0.05$, $* * P<0.01$, $* * * P<0.005$; NS, not significant. Scale bars: $200 \mu \mathrm{m}$ and $100 \mu \mathrm{m}$ (higher magnification.

A

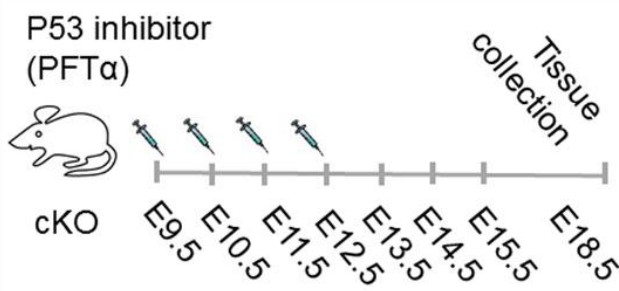

B

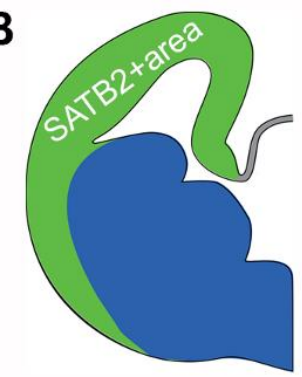

C

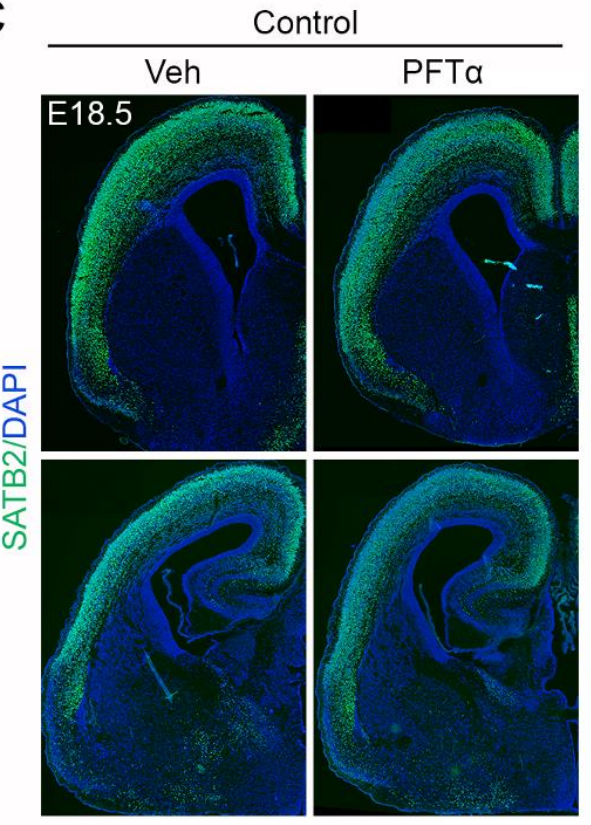

G

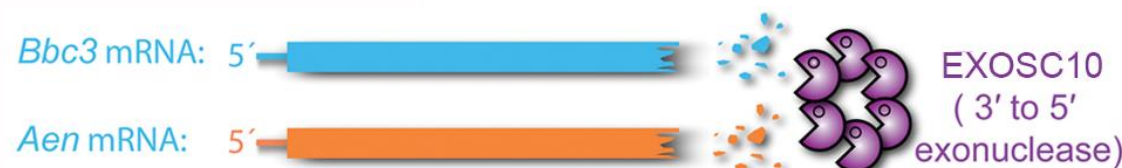

Wild-type

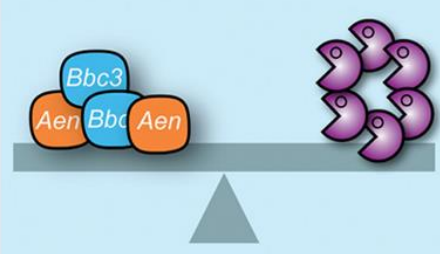

P53 signaling activity: low

Cell survival
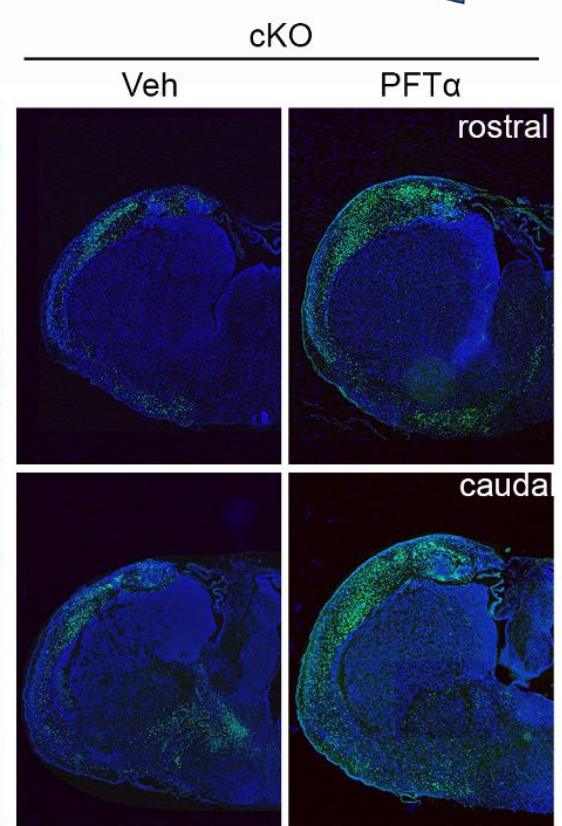

$\mathbf{F}$

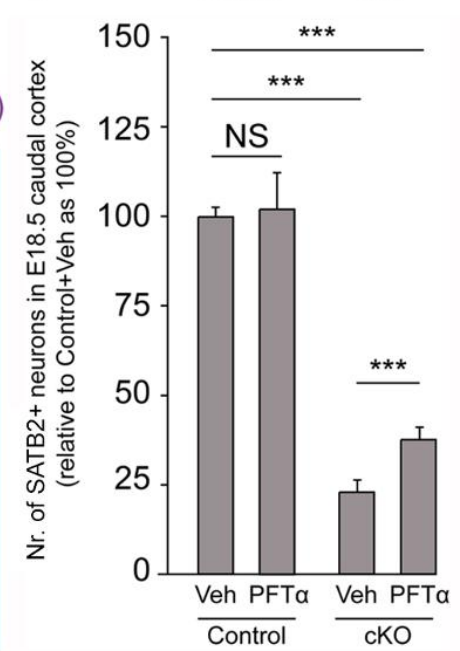

Figure 3.8 EXOSC10 controls cortical development partly by suppressing P53 signaling activity.

(A,B) Rescue experimental paradigm (A) to examine effect of P53 signaling inhibitor (PFT $\alpha$ ) on the SATB2+ cortical area (B). (C-F) Immunohistochemistry with SATB2 antibody (C) and quantitative analyses (D-F) of cKO mutants at E18.5, showing the effects of treatment with PFT $\alpha$ on the SATB2+ cortical area at rostral (D) and caudal (E) levels, and on the pool of SATB2+ projection neurons at the caudal level (F). (G) A proposed model showing how the balanced level of RNA exonuclease EXOSC10 and the P53 signaling factors AEN and BBC3 ensures cell survival, whereas the loss of EXOSC10 causes accumulation of AEN and BBC3, leading to apoptosis. $* P<0.05, * * P<0.01$, and $* * * P<0.005$; NS, not significant. Scale bar: $200 \mu \mathrm{m}$. 


\subsection{Discussion}

Here, we have investigated the possible function of the RNA exonuclease EXOSC10 in brain development. We demonstrate that expression of EXOSC10 is crucial for controlling cell survival and cortical development. Our findings indicate that EXOSC10 performs an essential function in controlling P53-mediated apoptosis signaling by directly degrading the P53 signaling-related transcripts such as AEN and BBC3 (Fig. 3.8G).

\subsubsection{Emerging roles of RNA stability regulation in brain development}

Post-transcriptional regulations such as RNA modification and RNA stability are emerging as mechanisms that are essential for regulation of gene expression. The best known mechanism of RNA modification, so far, is the methylation of nitrogen 6 in adenosine (N6-methyladenosine, m6A). The m6A writer complex, consisting of RBM15, WTAP, METTL3 and METTL14, is responsible for addition of methyl groups to RNA (Liu et al., 2014; Wang et al., 2016). On the other hand, the m6A eraser Alkbh5 can remove methyl groups installed on RNA (Zheng et al., 2013). YTH proteins and eIF3 can serve as m6A readers to recognize m6A (Meyer et al., 2015; Patil et al., 2016). Recent studies suggest important roles for m6A methylation in the modulation of RNA stability in brain development (Flamand and Meyer, 2019; Li et al., 2018b; Wang et al., 2018; Widagdo and Anggono, 2018; Yoon et al., 2017).

m6A deficiency by METTL14 cKO in the developing mouse brain increased the stability of NSC transcripts, causing lengthening of the cell cycle of NPCs and prolongation of cortical neurogenesis further at postnatal stage (Yoon et al., 2017). The m6A depletion by METTL14 cKO also changed the levels of modified histones and of transcripts encoding histone-modifying enzymes, indicating that m6A-dependent control of epigenetic program alterations is involved in neurodevelopment (Wang et al., 2018).

Deletion of m6A reader YTHDF2 in YTHDF2 KO mice leads to late embryonic lethality. Neurogenesis was declined significantly with concurrent reduction in the number of TBR2+ BPs leading to a thinner cortical plate in YTHDF2 KO embryos (Li et al., 2018b). It should be noted, however, that m6A was shown to impact not only RNA stability but also other features of RNA metabolic processes, such as translation, splicing and transport of RNA (Dominissini et al., 2012; Meyer et al., 2012; Wang et al., 2014; Wang et al., 2015; Xiao et al., 2016). Thus, the precise roles of RNA stability in brain development are still largely unknown.

Functional investigations of genes encoding exosome subunits in model systems indicated that most exosome subunits are required for viability from yeast to man (Fasken et al., 2020; Januszyk and Lima, 2014; Kilchert et al., 2016; Morton et al., 2018). Although the precise role of the exosome complex in neural development is not known, mutations in four out of the 11 exosome subunit genes in humans have been found to be associated with neurodevelopmental and psychiatric disorders. Notably, mutations in EXOSC2 are associated with intellectual disability (Di Donato et al., 2016), whereas mutations in EXOSC3 (Eggens et al., 2014; Halevy et al., 2014; Rudnik-Schoneborn et al., 2013; Wan et al., 2012; Zanni et al., 2013), EXOSC8 (Boczonadi et al., 2014) and EXOSC9 (Burns et al., 2018) cause different types of cerebellar hypoplasia that lead to severe neurodegeneration and lethality. Mutations of these exosome factors were also associated with other brain defects, such as corpus callosum 
hypoplasia, cerebellar atrophy and abnormal myelination, as well as pontocerebellar hypoplasia with cerebellar and spinal motor neuron degeneration (Boczonadi et al., 2014; Burns et al., 2018; Di Donato et al., 2016; Eggens et al., 2014; Halevy et al., 2014; Rudnik-Schoneborn et al., 2013; Wan et al., 2012; Zanni et al., 2013). The findings in these human genetics studies indicate that the RNA exosome is crucial for normal neural development and cognition (Fasken et al., 2020; Morton et al., 2018).

Among EXOSC subunits, the role of EXOSC10 in biological processes is the most investigated. EXOSC10 has been shown to stimulate mRNA turnover (van Dijk et al., 2007), 3' pre-rRNA processing (Knight et al., 2016), and decay of long non-coding and enhancer RNAs (eRNAs and lncRNAs) (Pefanis et al., 2015) with its absence causing RNA processing defects in yeast (Carneiro et al., 2007) and increased vulnerability to DNA damage (DomingoPrim et al., 2019; Marin-Vicente et al., 2015; Rolfsmeier et al., 2011). EXOSC10 functions with a co-factor, such as the NEXT complex, that recognizes and degrades RNA in DNA/RNA hybrid or RNA/RNA hybrid configuration, or eRNAs/lncRNAs (Lim et al., 2017; Lubas et al., 2011; Puno and Lima, 2018; Schmid and Jensen, 2019). Studies in cultured cell lines and in transgenic mice show that human EXOSC10 is crucial for cell cycle (Blomen et al., 2015). More recent in vivo work reported that EXOSC10 controls the onset of spermatogenesis in male germ cells (Jamin et al., 2017). Accordingly, EXOSC10cKO mutant mice show small testes and impaired differentiation of germ cells, and exhibit reduced fertility (Jamin et al., 2017). However, whether or not EXOSC10 is essential for brain development has remained unclear. Our findings reveal that the function of EXOSC10 is required for the development of the forebrain. During early corticogenesis, EXOSC10 is indispensable for cell viability and cortical layer formation. The requirement of the exosome complex in cell survival identified using the EXOSC10cKO_Emxl-Cre cortex could possibly explain the aforementioned neurodegeneration caused by mutations of human EXOSC genes.

The ring-like structured exosome complex contains eleven evolutionarily conserved subunits, including nine structural subunits (EXOSC1-9) and two catalytic subunits (EXOSC10 and DIS3) (Januszyk and Lima, 2014; Kilchert et al., 2016). The expression pattern analysis (Fig. S3.1B-J) revealed that many exosome subunits (e.g. EXOSC1, EXOSC2, EXOSC3, EXOSC5, EXOSC9 and EXOSC10) are widely expressed in developing mouse cortex. Remarkably, expression of some subunits is found to be limited to the VZ (EXOSC8) or SVZ (EXOSC7). This raises the question of whether all the components are required for the RNA exonuclease activity of the exosome complex. Even though our understanding of the functions of the exosome complex and its subunits in development has improved, several key questions remain unanswered. For example, is the composition of the exosome complex restricted to eleven subunits? Also, what is the contribution of individual subunits in formation and action of the exosome complex? Whether lineage-restricted subunits exist that lead to dynamic combinatorial assembly of exosome complexes, producing their biological specificity, remains to be determined. Efforts to resolve these and other questions would stimulate continuous interest in this area of research. 


\subsubsection{P53 pathway genes Aen and Bbc3 are targets of EXOSC10 in developing cortex}

P53 is a well-known master regulator of numerous developmental events. It triggers expression of various downstream genes, some of which promote growth arrest and DNA repair, whereas others are involved in apoptosis (Jain and Barton, 2018; Mendrysa et al., 2011). The massive apoptosis observed in the early developing cortex of EXOSC10cKO_Emxl-Cre embryos culminated in a severe reduction of cortical size.

To gain a transcriptome-wide insight into the role of exosome complex in corticogenesis, we carried out RNA-seq analysis of cKO cortices and EXOSC10 RIP-seq. Our findings revealed upregulation of several genes associated with the P53 apoptosis pathway. Interestingly, cardinal components of apoptosis-associated P53 signaling, AEN (Kawase et al., 2008) and BBC3 (Han et al., 2001; Jeffers et al., 2003), were identified in our RNA-seq and RIP-seq analyses. The RNA degradation assay carried out further highlighted that EXOSC10 directly degrades transcripts of AEN and BBC3.

AEN possesses exonuclease activity to degrade both DNA and RNA (Lee et al., 2005). In P53 signaling-dependent apoptosis, DNA damage signals lead to translocation of AEN into the nucleolus, causing nucleolar disruption (Kawase et al., 2008). Subsequently, AEN degrades DNA and RNA, amplifying the damage signal and inducing apoptosis (Kawase et al., 2008). The promoter region of BBC3 contains P53-binding sites and can be directly activated by P53 (Han et al., 2001). BBC3 is part of the BH3-only BCL-2 family proteins, which have been found to localize to mitochondria in response to apoptotic stimuli, where they induce mitochondrial apoptosis (Huang and Strasser, 2000; Lomonosova and Chinnadurai, 2008). BBC3 has been shown to be required for $\gamma$-irradiation-induced cell death in the developing brain, and P53 is not able to induce apoptosis in the absence of BBC3 (Jeffers et al., 2003). Our data also highlight that EXOSC10 regulates cell viability in developing cortex by repressing the distinct P53-dependent apoptosis signaling pathways, including those caused by DNA/RNA damage and $\gamma$-irradiation signals. Notably, PFT $\alpha$ treatment significantly rescues the aberrant upregulation of BBC3 (but not AEN) upon the loss of EXOSC10 in the developing cortex (Fig. 3.7D,E). This proves that BBC3 (but not AEN) is a direct target of P53.

Previous studies indicated that accumulation of RNA/DNA hybrids or noncoding RNAs as eRNAs/lncRNAs could induce cellular genomic instability leading to P53 activation and cell death (Pefanis et al., 2015; Wolin and Maquat, 2019). In addition, RNA exosome is important for DNA DSB repair as the lack of EXOSC10 leads to accumulation of DNA breaks and P53 activation (Domingo-Prim et al., 2019; Pefanis et al., 2015). The findings suggest that other mechanisms in addition to the increased expression of AEN and BBC3 cause the hyperactivation of P53 signaling in response to the defect of RNA exosome activity.

In addition to the apoptotic P53 signaling pathway, we examined the oxidative stress signaling - one of the well-known cell death-triggering pathways in the developing brain (Green, 1998; Ikonomidou, 2009). Expression of genes encoding the main components of this pathway (e.g. BAX, BH3 and Cytochrome C) was unchanged in our RNA-seq analysis, and the pathway itself was not found in a corresponding GO study (Tables S1 and S2). Thus, our findings suggest that EXOSC10 inhibits apoptosis mainly by suppressing the activity of the apoptotic P53 signaling pathway. 
Pharmacological inhibition of P53 signaling rescued the described defects in cell viability in the EXOSC10cKO mutants (Fig. 3.7A-C), suggesting that the EXOSC complex negatively regulates P53 signaling during early cortical development. It is worth noting, however, that the inhibition of P53 signaling was not able to restore the normal thickness of cortical layers in EXOSC10cKO mutants (Fig. 3.7A-C). Therefore, the observed drastic reduction in the size of the cortical plate cannot be singularly ascribed to the increased apoptosis but other unreported perturbations may contribute to the observed defective neurogenesis. In agreement with this assertion, our RNA-seq and RIP-seq data suggest that EXOSC10 might directly suppress expression of many neuronal differentiation-associated genes. Possible defects in neurogenesis and neuronal differentiation in the cortex-specific EXOSC10cKO mutants will be in focus in a separate study.

Overall, our findings indicate a crucial role for EXOSC10 in P53 pathway-mediated apoptosis, in which the binding of EXOSC10 to the mRNAs of the P53 signaling mediators AEN and BBC3 confers their rapid turnover. Our study indicates that suppression of P53 signaling by the exosome complex is essential for normal cell survival and brain development (Fig. 3.8G).

\subsection{Materials and Methods}

\subsubsection{Transgenic mice}

Conditional inversion (COIN) alleles for EXOSC10 (COIN/COIN) (Economides et al., 2013; Pefanis et al., 2015), FoxG1-Cre (Hebert and McConnell, 2000) and Emxl-Cre (Gorski et al., 2002) mice (Mus musculus) were maintained in a C57BL6/J background. Animals were handled in accordance with the German Animal Protection Law.

\subsubsection{Antibodies}

A list of antibodies is provided in the supplementary Materials and Methods.

\subsubsection{Immunohistochemistry, western blotting and qPCR}

Detailed descriptions have been provided previously (Narayanan et al., 2015) and more detail can be found in the supplementary Materials and Methods.

\subsubsection{RNA-sequencing (RNA-seq), RNA-immunoprecipitation sequencing (RIP-seq) and bioinformatics analyses}

Detailed descriptions have been provided previously for RNA-seq (Narayanan et al., 2015; Nguyen et al., 2018), RIP-seq (Xie et al., 2019; Yoon et al., 2017) and bioinformatics analyses (Narayanan et al., 2015; Nguyen et al., 2018). In RNA-seq experiments, RNA was obtained from cortex from five control and five EXOSC10cKO embryos at E12.5. cDNA libraries were prepared using the TruSeq RNA Sample Preparation v2 Kit. DNA was quantified using a Nanodrop spectrophotometer, and its quality was assessed using an Agilent 2100 Bioanalyzer. 
EXOSC10 RNA-seq was performed using Magna RIP Kit (Merck Millipore) according to the manufacturer's instructions. In brief, dissociated cells from E12.5 cortex on $10 \mathrm{~cm}$ dish were lysed in $400 \mu \mathrm{l}$ of complete RIP lysis buffer-containing protease inhibitors and RNase inhibitor. EXOSC10 protein was pulled down using a Dynabeads-associated EXOSC10 antibody. A mock pull-down was carried out with normal rabbit IgG (Cell Signaling Technologies). The immunoprecipitated complex was washed intensively and the pulled down RNA was extracted using Trizol reagent. Purified RNA was sequenced at the Transcriptome and Genome Analysis Laboratory (TAL) (University of Goettingen, Germany).

Data obtained from RNA- and RIP-seq were processed with the help of the Galaxy web platform (Afgan et al., 2018) and further analyzed using Webgestalt (http://www.webgestalt.org) (Wang et al., 2013; Wang et al., 2017; Zhang et al., 2005) and DAVID Bioinformatics Resources 6.8 (Huang da et al., 2009). Gen sets from RNA- and RIPseq were compared using Venny 2.1 (Oliveros). Base calling, fastq conversion, quality control and read alignments were all achieved as outlined for RIP-Seq. Reads were aligned to mouse genome mm10 and counted using FeaturesCount (http://bioinf.wehi.edu.au/featureCounts/). Differential expression was assessed using DESeq2 from Bioconductor (Love et al., 2014). Functional GO enrichment analyses were performed using ToppGene (Chen et al., 2009).

\subsubsection{Culture and generation of EXOSC10 KO primary NSCs}

Mouse NSCs were isolated from E12.5 EXOSC10 COIN/COIN cortices and cultured in NSC culture medium containing KO DMEM/F12 (Invitrogen), StemPro Neural Supplement (Invitrogen), Glutamax (Invitrogen), penicillin/streptomycin (Invitrogen), $20 \mathrm{ng} / \mathrm{ml} \mathrm{FGF2}$ (Invitrogen) and $20 \mathrm{ng} / \mathrm{ml}$ EGF (Invitrogen) on culture dishes precoated with $0.1 \%$ gelatin as described previously (Tuoc et al., 2013; Tuoc and Stoykova, 2008). TAT-Cre recombinase (Excellgen) $(1 \mu \mathrm{M})$ was added to fresh NSC culture medium for $26 \mathrm{~h}$ to achieve EXOSC10 KO.

\subsubsection{RNA degradation assay}

The assay was performed as described previously (Xie et al., 2019; Yoon et al., 2017). Briefly, EXOSC10 COIN/COIN mouse NSCs were cultured to about 70-90\% confluence. Some wells were treated with $1 \mu \mathrm{M}$ TAT-Cre (Excellgen) $26 \mathrm{~h}$ beforehand to achieve EXOSC10 KO; cells with vehicle solution served as controls. Actinomycin D (5 mM, Sigma) was supplemented to fresh NSC culture medium. Subsequently, cells were harvested at different time points $(0 \mathrm{~h}, 5$ $\mathrm{h}$ and $17 \mathrm{~h}$ ) by washing once with PBS and detaching using Tripsin/ EDTA(Sigma). For transcript quantification, RNA was extracted from the NSC samples. qPCR was carried out to quantify the transcript level of target genes. The experiment was performed in triplicate and normalized to internal 18S. Fold changes of transcript targets between EXOSC10KO and control NSCs were compared at different time points $(0 \mathrm{~h}, 5 \mathrm{~h}$ and $17 \mathrm{~h})$ after actinomycin D treatment. Fold changes at $5 \mathrm{~h}$ and $17 \mathrm{~h}$ were normalized to those at $0 \mathrm{~h}$ (before actinomycin $\mathrm{D}$ treatment). 


\subsubsection{In vivo pharmacological treatment and rescue experiments}

EXOSC10cKO_Emxl-Cre mouse embryos were subjected to the P53- inhibitor Pifithrin- $\alpha$ (PFT- $\alpha$ ) (Komarov et al., 1999) by intraperitoneal injection of $2.2 \mathrm{mg} / \mathrm{kg}$ PFT- $\alpha$ (Selleckchem) into the pregnant mother at between E9.5 and E12.5 or between E9.5 and E15.5. Embryonic brains were isolated at E13.5 or E18.5 and immunohistochemistry was performed.

\subsubsection{Relative quantification of cortical size}

Dorsal views of forebrains of mutant and control mice were photographed under a dissection microscope. Cortical anterior-posterior axis (AP), cortical surface and midline lengths from the digitized images were measured with Fiji software to make comparison between mutants and controls. For further details, see the supplementary Materials and Methods.

\subsubsection{Cell counts and quantitative analysis of IHC signal intensity}

IHC quantification was performed using anatomically matched coronal sections. In most cases, cell counts of six matched sections were averaged (control/cKO). For quantitative analyses of IHC signal intensity of cytoplasm-staining markers, fluorescent images of selected areas of the cortex were used. Color images were converted to gray scale and the fluorescent signal intensity values were measured using the Analyze/Measure function of Fiji software. The signal intensity from the background next to the tissue was subtracted from the measured intensity for normalization. For further details, see the supplementary Materials and Methods.

\subsubsection{Image acquisition and statistical analysis}

Imaging was performed with an Axio Imager M2 (Zeiss) with a Neurolucida system (Version 11; MBF Bioscience) and a confocal fluorescence microscope (TCS SP5; Leica). Images were further analyzed with Adobe Photoshop and Fiji. Statistical analyses were carried out using Student's t-test. Graphs are plotted as mean \pm s.e.m. An unpaired t-test was carried out on the average from at least three biological replicates. All details of statistical analyses and description for histological experiments are presented in Table S7 and in the supplementary Materials and Methods.

\subsection{Acknowledgements}

We acknowledge T. Huttanus and M. Blessmann for their expert animal care and support. We also thank F. Guillemot, A. Nave, A. P. McMahon, A. Jones and O. Machon for providing reagents.

\subsection{Competing interests}

The authors declare no competing or financial interests. 


\subsection{Author contributions}

Conceptualization: T.T.; Methodology: Y.X., O.S., T.T.; Validation: T.T.; Formal analysis: P.A.U., G.S., L.P., O.S., J.R., T.B., A.F.; Investigation: P.A.U., Y.X., L.P., T.B., A.F.; Resources: U.B., H.P.N., J.F.S., T.T.; Writing - original draft: P.A.U.; Writing - review \& editing: G.S., T.T.; Supervision: T.T.; Project administration: T.T.; Funding acquisition: H.P.N., J.F.S., T.T.

\subsection{Funding}

This work was supported by the Deutsche Forschungsgemeinschaft (TU432/1-1, TU432/3-1 and TU432/6-1 to T.T.), by the Schram-Stiftung (T.T.) and by the National Institutes of Health/National Institute of Allergy and Infectious Diseases (AI134988 to U.B.). Deposited in PMC for release after 12 months.

\subsection{Data availability}

All RNA-seq and RIP-seq data have been deposited in GEO under accession number GSE164188.

\subsection{Supplementary figures}


A $5^{\prime}$

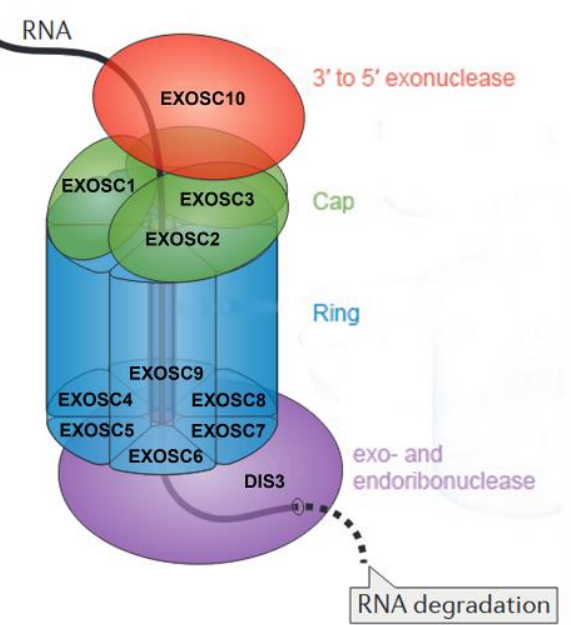

B EXOSC1

C

EXOSC2

D EXXSC3

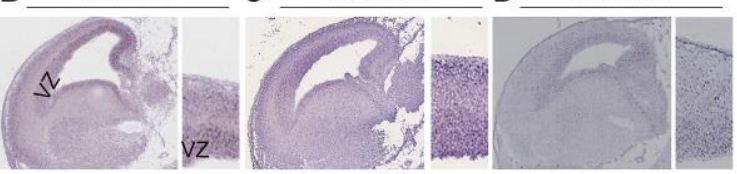

$\begin{array}{llllll}\mathbf{E} & \mathrm{EXOSC5} & \mathbf{F} & \mathrm{EXOSC6} & \mathbf{G} & \mathrm{EXOSC7}\end{array}$

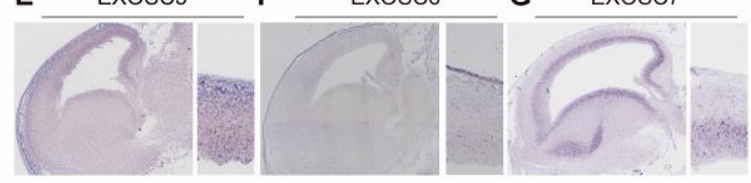

$\begin{array}{llllll}\mathbf{H} & \text { EXOSC8 } & \mathbf{I} & \text { EXOSC9 } & \mathbf{J} & \text { EXOSC10 }\end{array}$

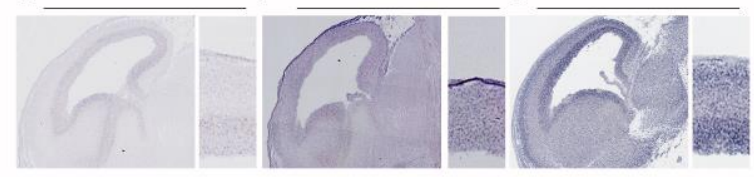

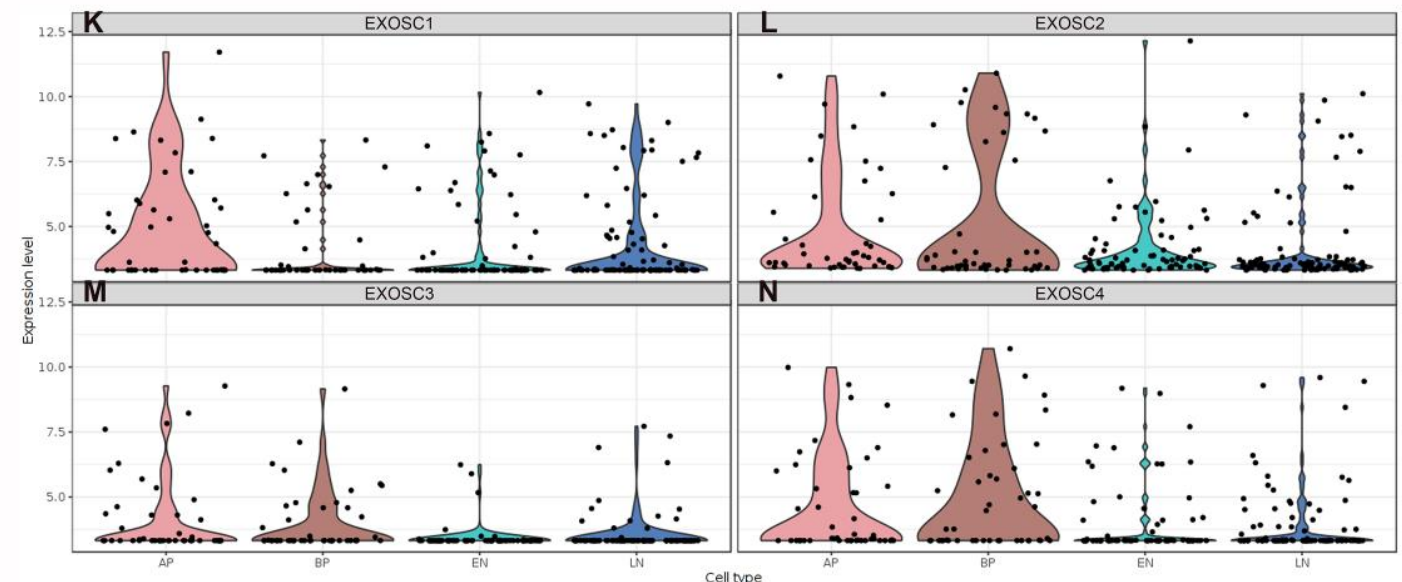

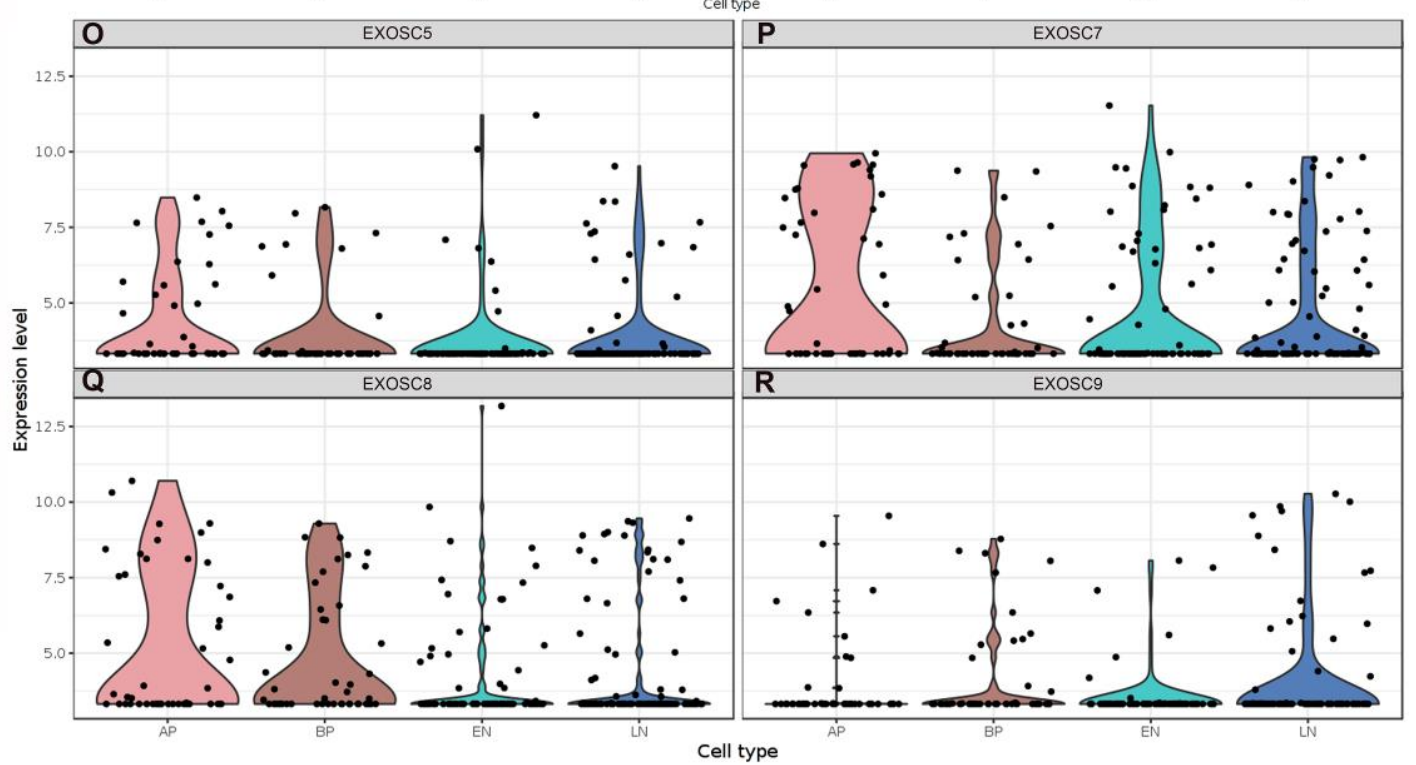

Figure S3.1 Expression of exosome complex subunits in the developing mouse brain.

(A) Model structure of the exosome complex with its components (Kilchert et al., 2016). (B-J) In situ hybridization of exosome complex components in sagittal sections of the E14.5 brains obtained from GenePaint database (Visel et al., 2004). Higher magnification of the cortex on right panels shows a high expression of exosome genes in the cortex, especially in the ventricular zone (VZ). (K-R) Expression of exosome subunits based on a published singlecell RNA-seq dataset of embryonic mouse cortex (Telley et al., 2016). The expression profiles of exosome subunits as violin plots was generated using the Seurat package of R (http://genebrowser.unige.ch/science2016/) (Macosko et al., 2015). AP: Apical progenitors/RGCs; BP: daughter basal progenitors/IPCs; EN: early-born neurons; LN: late-born neurons. 


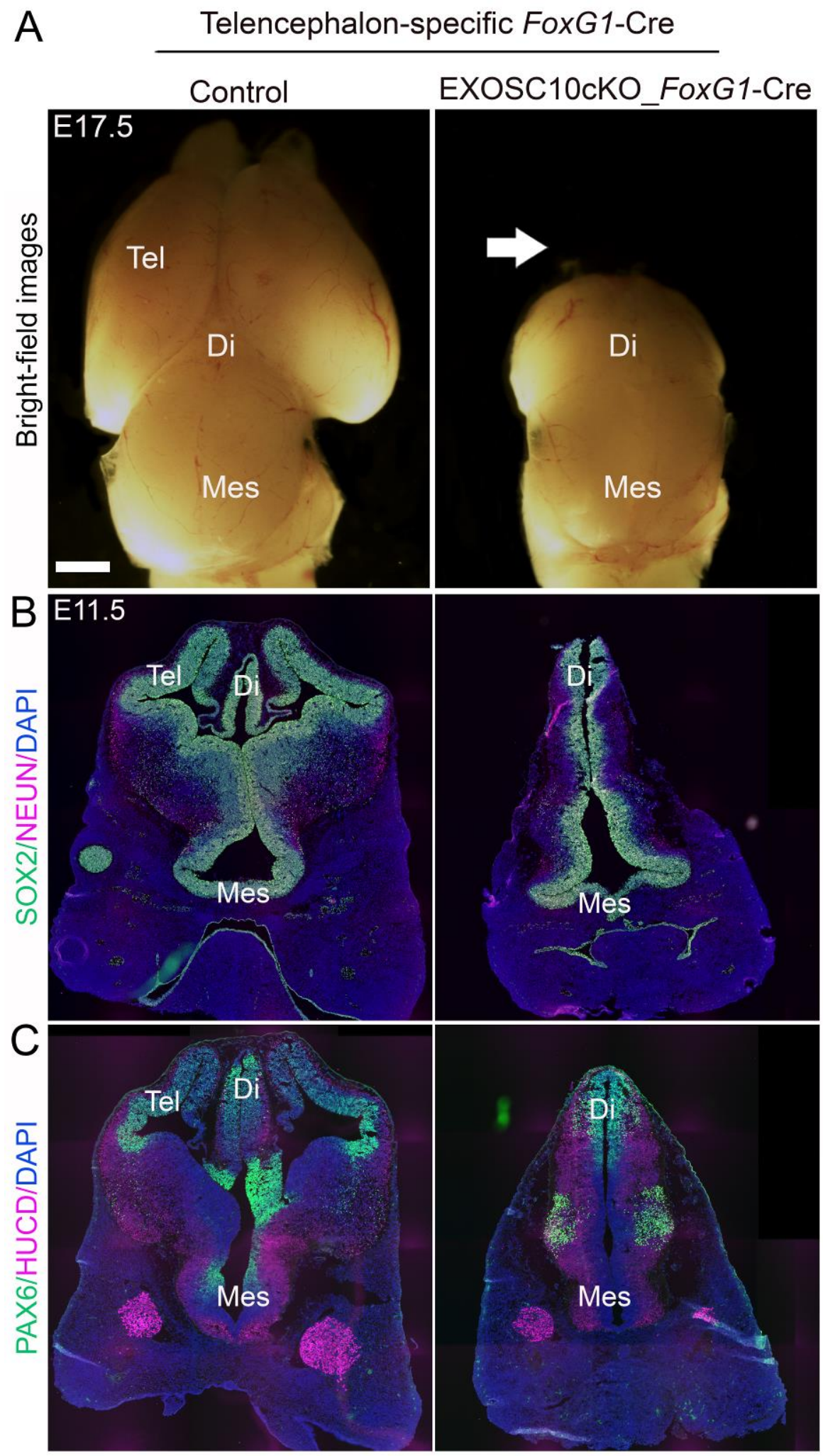

Figure S3.2 Expression of EXOSC10 is required for specification of telencephalon.

(A) The forebrain-specific cKO_FoxGl-Cre embryos have no telencephalon. (B, C) Representative images show IHC analyses with coronal sections of head from control and cKO_FoxG1-Cre embryos at E11.5 with antibodies against SOX2, NEUN (B) and PAX6, HUCD (C) that specifically label primordial NSC markers SOX2, PAX6, and immature neuronal markers NEUN, and HUCD respectively. IHC analyses revealed detectable SOX2+, PAX6+ NSCs and HUCD+, NEUN+ neurons in diencephalon (Di) and in mesencephalon (Mes) in both control and cKO head, whereas these cell types were detected only in dorsolateral part (telencephalon, Tel) of control, but not that of cKO head. Abbreviations: Tel, telencephalon; Di, diencephalon; Mes, mesencephalon. Scale bars $=1$ $\mathrm{mm}$. 


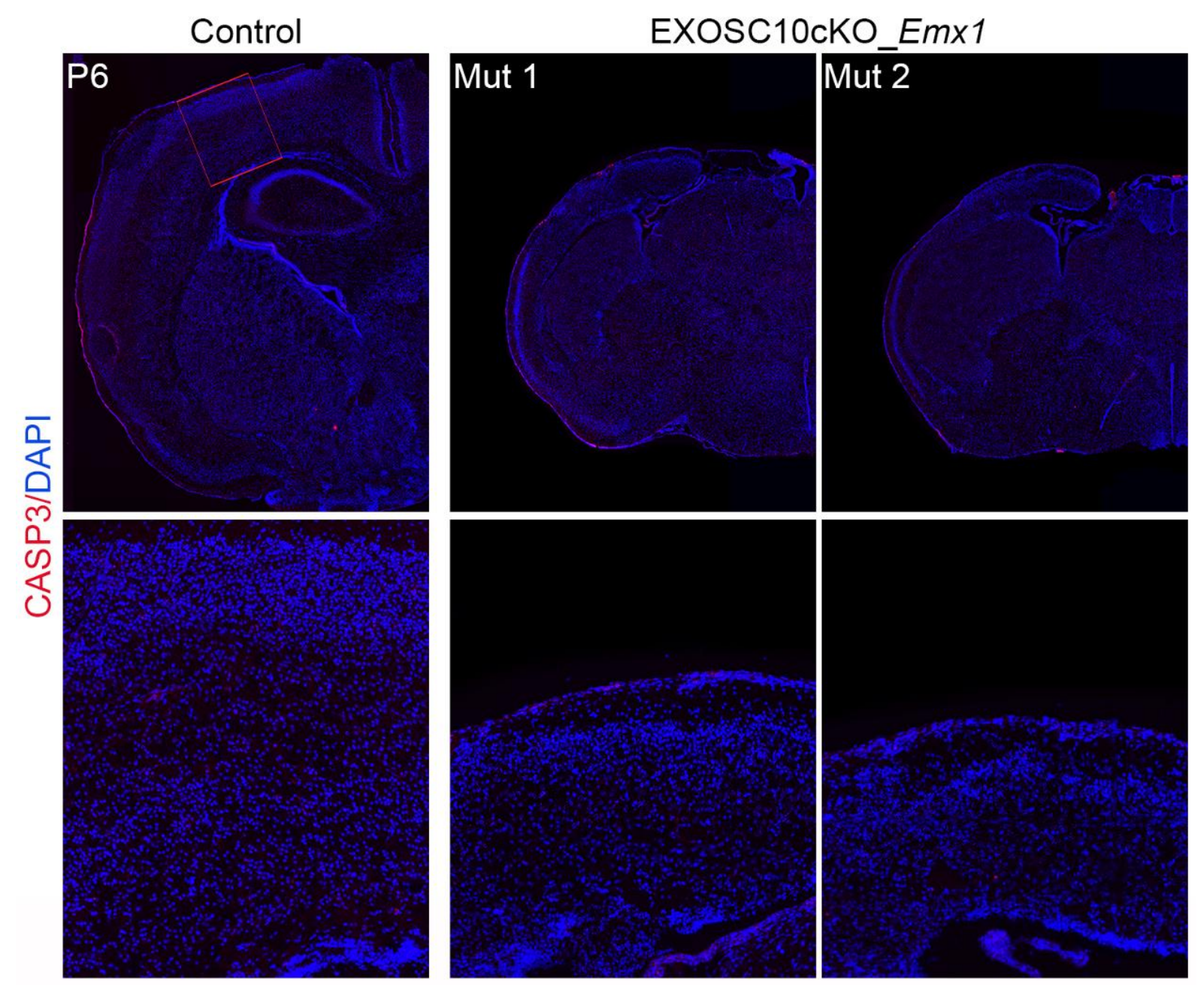

Figure S3.3 EXOSC10 expression is not required for cell viability in postnatal cortex.

IHC analysis with antibody against CASP3 to detect cell death (counter staining with DAPI) indicated there was no obvious difference in number of CASP3+ apoptotic cells between control and EXOSC10cKO cortices at P6. 
A

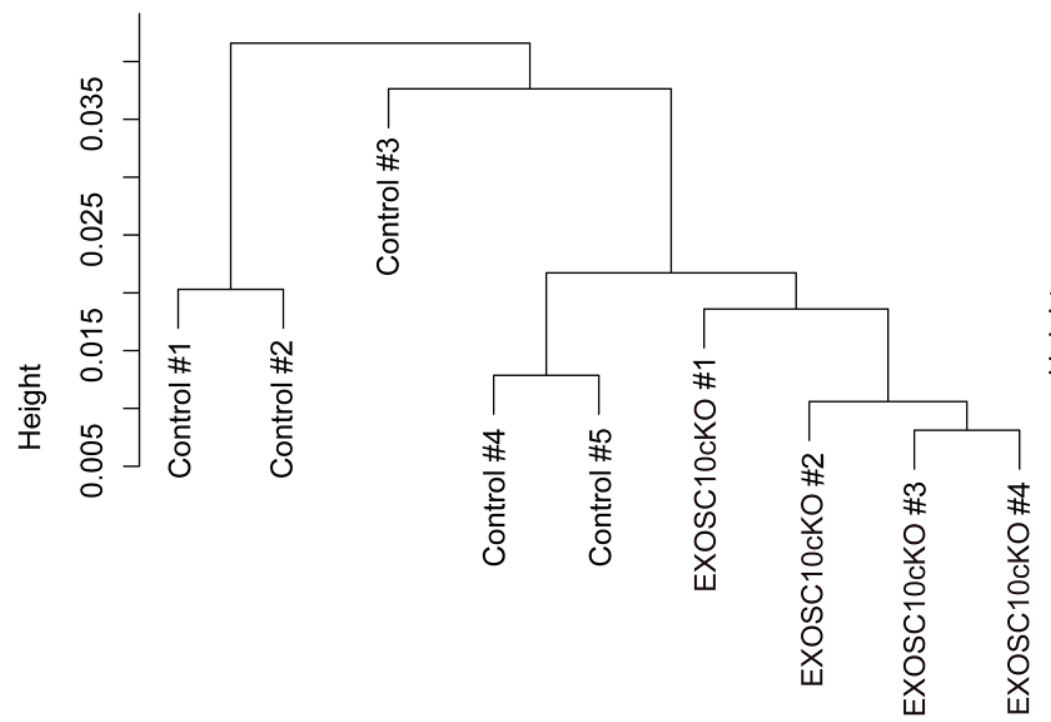

C Control EXOSC10cKO

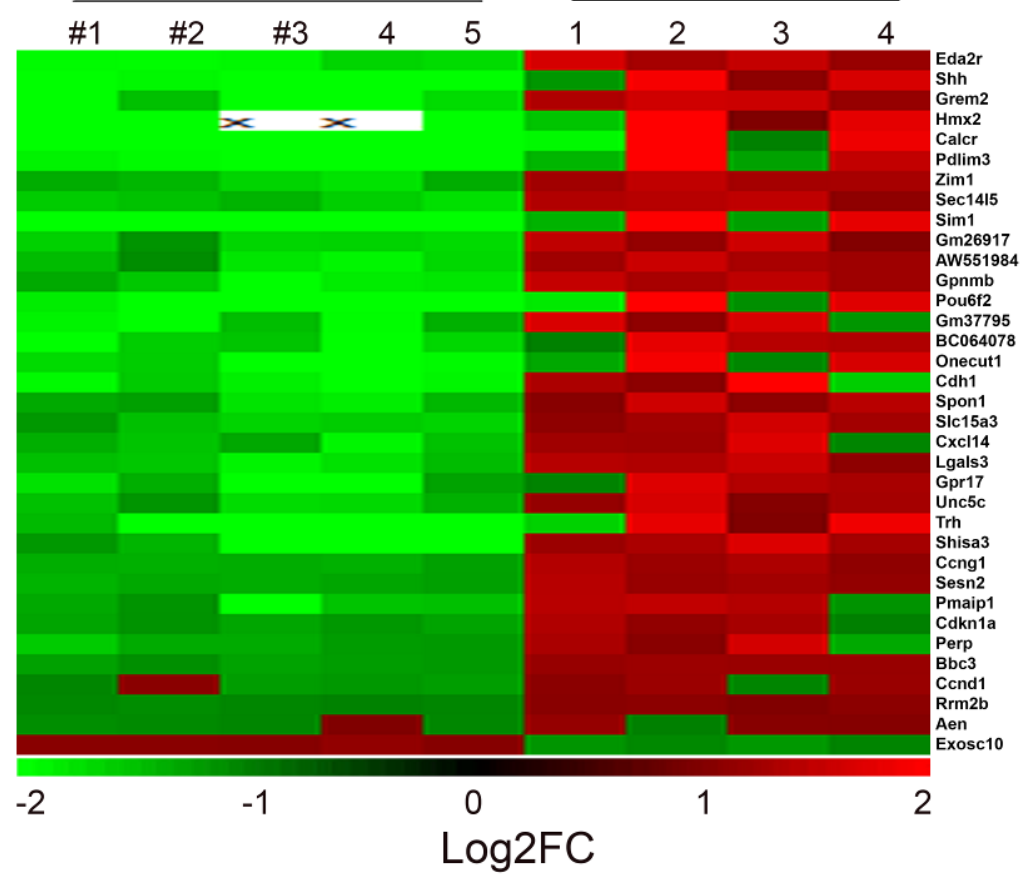

B

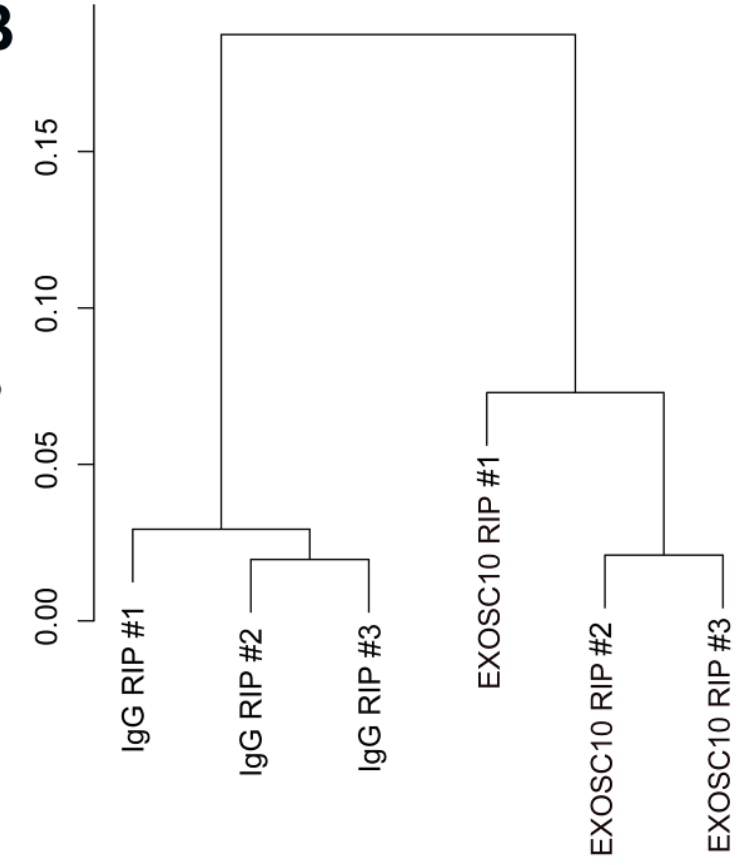

D IgG RIP EXOSC10 RIP

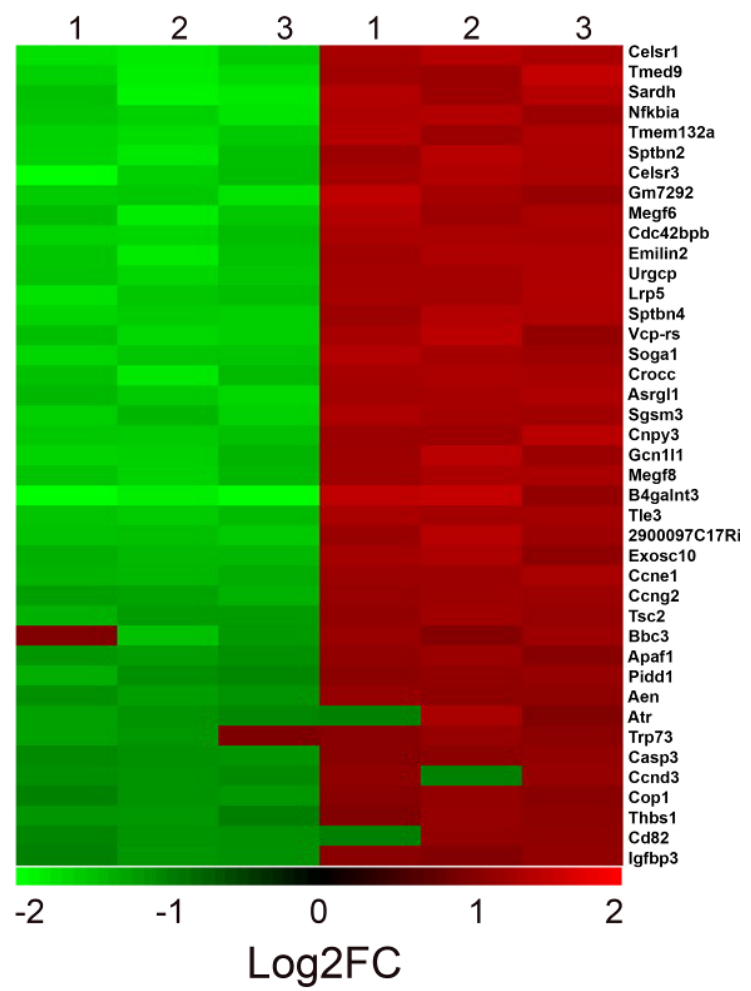

Figure S3.4 Cluster and Heatmap analyses of RNA-seq and RIP-seq samples.

IHC analysis with antibody (A, B) cluster dendrogram analysis of all RNA-seq (A, control, EXOSC10cKO) and RIP-seq (B, IgG RIP, EXOSC10 RIP) samples. The log2-nomalized values of all the genes were used for cluster analysis. (C, D) Heatmaps showing changes in gene expression (C) and transcript binding enrichment (D) revealed by RNA-seq (E12.5 EXOSC10cKO cortex vs. control) and RIP-seq (E12.5 cortex, EXOSC10 antibody vs IgG) analyses, respectively. Top 25 upregulated/enriched genes and genes involved in P53 apoptosis signaling were displayed. Additionally, EXOSC10 was shown as a control. 


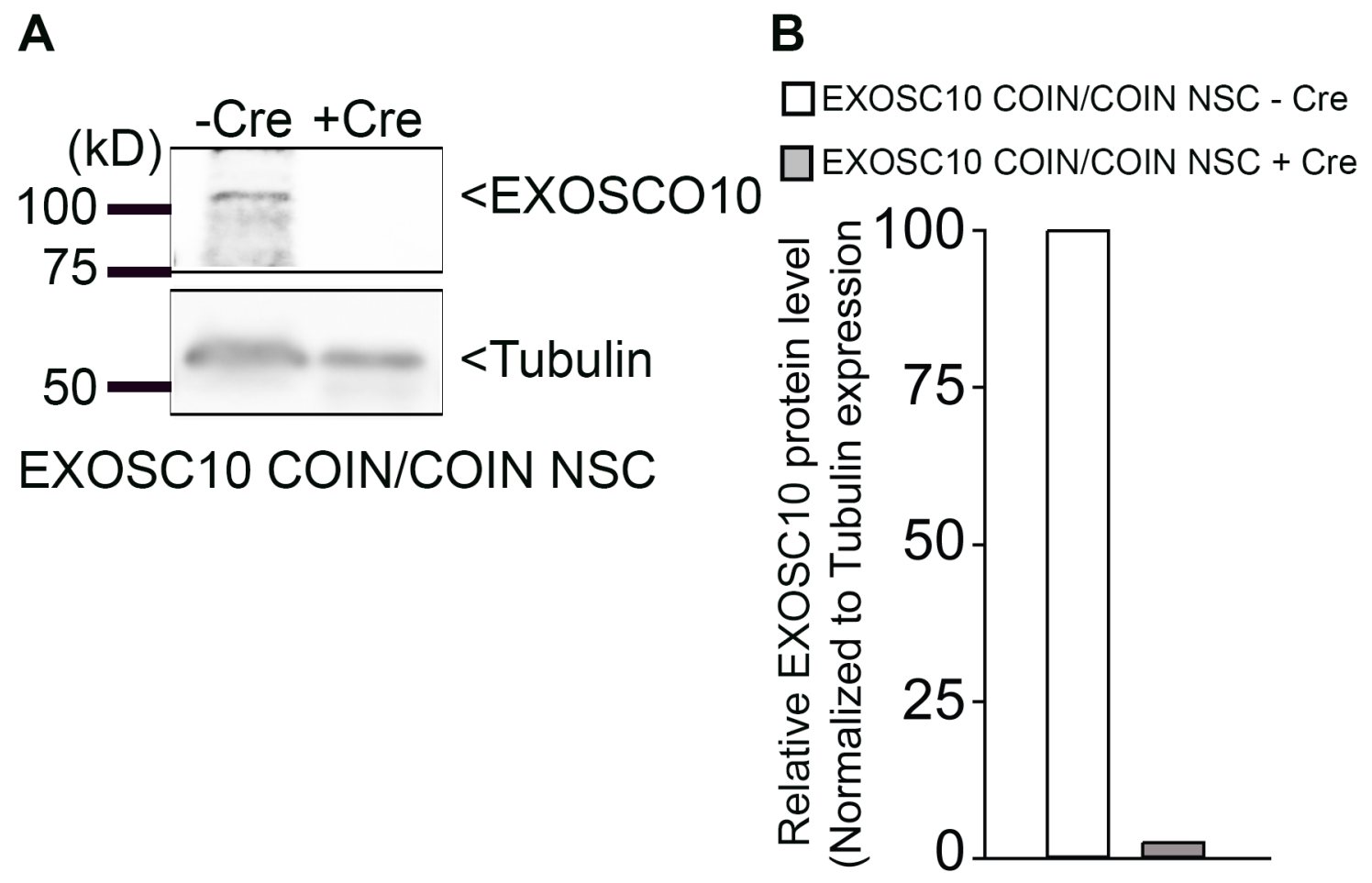

Figure S3.5 Knockout of EXOSC10 using soluble Tat-Cre recombinase in EXOSC10 ${ }^{\operatorname{CoIN} / C O I N}$ NSCs and the effects of PFTa treatment on BBC3 expression in the developing cortex.

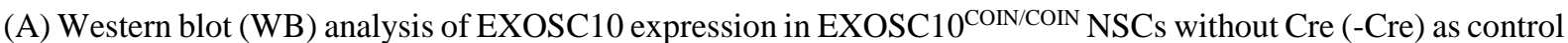
and after $26 \mathrm{~h}$ of treatment with soluble Tat-Cre recombinase (+Cre). Tubulin is shown as a loading control. (B) Quantification of the protein band densities (from A) relative to control (as 100\%), which was normalized to tubulin.

\subsection{Supplementary materials and methods}

\subsubsection{Antibodies}

Polyclonal $(\mathrm{pAb})$ and monoclonal $(\mathrm{mAb})$ primary antibodies used in this study (working dilution; sources): AEN rabbit pAb (1:400; Bioss), Caspase-3 rabbit pAb (1:200; Cell Signaling), CTIP2 rat mAb (1:200; Abcam), CUX1 rabbit pAb (1:50; Santa Cruz), EXOSC10 rabbit pAb (1:500 for WB; Proteintech), EXOSC10 rabbit pAb $(5 \mu \mathrm{g}$ for $100 \mu 1$ RIP-sample; Abcam), GFP chick pAb (1:400; Abcam), HUCD mouse mAb (1:20; Thermo Fisher Scientific), NEUN mouse mAb (1:200, Chemicon), REELIN mouse mAb (1:100), SATB2 mouse mAb (1:200; Abcam), SOX2 mouse mAb (1:100; Santa Cruz), SOX2 rat mAb (1:100; eBioscience), SOX5 rabbit pAb (1:100; Santa Cruz), TBR1 rabbit pAb (1:300; Abcam), TBR2 rabbit pAb (1:300; Chemicon), TBR2 rat mAb (1:200; eBioscience), TUJ mouse mAb (1:500; Chemicon), PAX6 mouse mAb (1:100; Developmental Studies Hybridoma Bank). Secondary antibodies used were Alexa 488-, Alexa 568-, Alexa 594- and Alexa 647-conjugated IgG (various species, 1:400; Molecular Probes).

\subsubsection{Relative quantification of cortical size}

Relative quantification of cortical size was performed as described previously (Narayanan et al., 2018; Tuoc et al., 2013). Briefly, dorsal views of forebrains of mutant and control mice 
were photographed under a dissection microscope. Cortical anterior-posterior axis (AP), cortical surface and midline lengths from the digitized images were measured with Fiji software to make comparison between mutants and controls.

\title{
3.12.3 Immunohistochemistry (IHC)
}

IHC was performed as described previously (Tuoc et al., 2009). Briefly, embryonic and postnatal brains were fixed in $4 \%$ paraformaldehyde, incubated in $25 \%$ sucrose in PBS-DEPC at $4{ }^{\circ} \mathrm{C}$ overnight, embedded in Tissue-Tek O.C.T Compound (Sakura Finetek) on dry ice and cut in $8-16 \mu \mathrm{m}$ coronal sections. After blocking with 5\% goat or donkey normal serum, sections were incubated overnight with primary antibody at $4{ }^{\circ} \mathrm{C}$ and the signal was detected with a fluorescent secondary antibody (Alexa Fluor; 1:400; Invitrogen). Sections were later counterstained with Vectashield mounting medium containing 4'-6'-diamidino-2-phenylindole (DAPI) (Vector Laboratories) to label nuclei.

\subsubsection{Quantitative RT-PCR (qPCR) and Western blot analyses}

qRT-PCR and Western blot analyses were performed as described previously (Tuoc and Stoykova, 2008). Primers used for qPCR:

\author{
AEN \\ Forward: 5'-GGCCGGGTTTTCAGATCCTTA-3' \\ Reverse: 5'- GCTGAGCAGGTTTGGGACAT-3' \\ BBC3 \\ Forward: 5'-ACGACCTCAACGCGCAGTA-3' \\ Reverse: 5'-CTAGTTGGGCTCCATTTCTGG-3' \\ Atr \\ Forward: 5'-AGGACACTCCAAAGCACCACTG-3' \\ Reverse: 5'-GCAGCCCTGTTACTCTATTTCGG-3' \\ $18 \mathrm{~S}$
}

Forward: 5'-CTTAGAGGGACAAGTGGCG-3'

Reverse: 5'-ACGCTGAGCCAGTCAGTGTA-3'

\subsubsection{Cell counts and quantitative analysis of IHC signal intensity}

IHC quantification was performed using anatomically matched coronal sections. Nucleus marker-positive cells were counted using the Cell Counter plugin of Fiji (Schindelin et al., 2012). In most cases, cell counts of six matched sections were averaged (control/cKO). The number of lineage marker cells was quantified using the total marker-positive cells in a defined region (radial unit) alone, or by normalizing to the total number of DAPI+ (nucleus-stained) cells using the following equation: Normalized number = marker-positive cell number/DAPI+ cell number. For quantitative analyses of IHC signal intensity of cytoplasm-staining markers, fluorescent images of selected areas of the cortex were used. Color images were converted to gray scale and the fluorescent signal intensity values were measured using the Analyze/Measure function of Fiji software. The signal intensity from the background next to the tissue was subtracted from the measured intensity for normalization. The normalized intensities of cKO 
sections are displayed relative to normalized values from control experiments as a percentage. Statistical comparisons of histological data were performed using Student's t-test. All bar graphs are plotted as means \pm SEM. All statistical tests are two-tailed, and P-values are considered to be significant for $\mathrm{p}<0.05(* \mathrm{p}<0.05, * * \mathrm{p}<0.01$, and $* * * \mathrm{p}<0.005$; NS, not significant).

\subsection{Additional supplementary information}

Supplementary information available online at https://dev.biologists.org/lookup/doi/10.1242/dev.188276.supplemental 


\section{Chapter 4: General Discussion}

In these studies, we investigated different mechanisms involved in cortical formation. First, we performed molecular profiling for IPCs and found several groups of genes specifically enriched in this highly relevant cell type. We further investigated the role of one of them, ESCO2, which is essential for sister chromatid cohesion during cell division.

Second, we examined the function of the RNA exonuclease EXOSC10 in cortical development. We show its role in regulating apoptosis and indicate further functions, which will be subject of future studies.

\subsection{ESCO2 and other IPC-related factors in cortical (mal)formation}

Identification of cell type-specific genes by transcriptome analysis of molecularly sorted cells can improve the understanding of the molecular landscape of distinct cell lineages. Here, we demonstrate the molecular profiling of the evolutionarily and clinically significant IPCs in the embryonic mouse cortex. We found a strong correlation of the identified IPC genes and known human developmental disorders, corresponding to the crucial functions of IPCs in cortical development.

A large proportion of cortical neurons originate from IPCs (Haubensak et al., 2004; Kowalczyk et al., 2009; Miyata et al., 2004; Noctor et al., 2004). These IPC-derived neurons form the upper cortical layers with their axons establishing the interhemispheric commissural system (i.e., the corpus callosum) (Fame et al., 2011). Evolutionary changes in the neurogenic output of IPCs have been linked to cortical expansion, especially seen in humans (MartinezCerdeno et al., 2006). Thus, disruption in the production, differentiation and maintenance of IPCs can result in various cortical malformations and diverse neurological perturbations.

Our GO analysis linked IPC-associated genes to neurodevelopmental defects, e.g. cortical size-related disorders (microcephaly, macrocephaly, and abnormal cortical gyration), corpus callosum defects (dysplastic, agenesis, aplasia, hypoplasia of corpus callosum, and abnormality of the cerebral white matter), and neurological deficits (intellectual disability, psychomotor developmental delay, schizophrenia, autism, and epilepsy).

Overall, our molecular profiling data offer a resource for future investigations to increase our knowledge about the contribution of IPC-associated genetic factors in cortical formation and their role in neurological disorders.

We next investigated the role of ESCO2, one of the IPC-related genes we identified by transcriptome analysis, in cortical development.

Previous studies in yeast, primary mouse embryonic fibroblasts and human cells revealed that ESCO2 is essential for sister chromatid cohesion (Hou and Zou, 2005; Terret et al., 2009; Vega et al., 2005; Whelan et al., 2012a; Whelan et al., 2012b). ESCO2 mediates appropriate cohesion of sister chromatids by the acetylation of cohesin. Dysfunction of ESCO2 leads to loss of cohesion, resulting in defective cohesin localization on chromosomes and finally causing apoptosis (Hou and Zou, 2005; Terret et al., 2009; Vega et al., 2005). Enriched ESCO1 expression was found in RGCs in the ventricular zone and enriched ESCO2 expression in IPCs in the subventricular zone of the developing mouse cortex. Hence, the cohesin 
acetyltransferases ESCO1 and ESCO2 seem to be cell viability determinants by ensuring correct tethering of sister chromatids in RGCs and IPCs, respectively. Indeed, our studies show that ESCO2 is substantial for IPC maintenance.

Studying a mouse model for the novel IPC gene ESCO2, we found that IPC survival and maintenance in the developing cortex depend on ESCO2. ESCO2 is crucial for the correct segregation of chromatids and therefore the distribution of genetic material into the progenies of dividing IPCs. Its loss may have caused the massive apoptosis of ESCO2-deficient IPCs and the resultant cortical dysgenesis.

Interestingly, ESCO2 mutations in human have been linked to the malformation syndromes RBS and JHS, which both include microcephaly and cognitive deficits (Kantaputra et al., 2020a; Kantaputra et al., 2020b; Van Den Berg and Francke, 1993; Vega et al., 2010). Karyograms from affected individuals show a characteristic heterochromatin puffing in the metaphase chromosomes resulting from impaired cohesion (Freeman et al., 1974; German, 1979; Van Den Berg and Francke, 1993).

Our study indicates that IPCs are particularly affected by the impaired cohesion. Previous studies reported that IPCs are transient amplifying progenitors in the developing cortex, undergoing multiple rounds of mitotic divisions (Hevner, 2019; Pontious et al., 2008). We found a high expression of numerous genes involved in cell cycle and chromatid segregation corresponding to the highly active cell division of IPCs. Thus, IPCs are especially vulnerable to loss of ESCO2 and the resulting impairment of cohesion, ultimately leading to apoptosis. The apoptosis of IPCs upon ESCO2 knockout and the subsequent failure in cortical formation seen in mice could also contribute to the microcephaly in humans with ESCO2 mutations.

\subsection{The exosome complex in cortical (mal)formation}

We showed for the first time the significance of the RNA exonuclease EXOSC10 in the development of the cortex. Loss of EXOSC10 at a very early embryonic stage (from E8.5), lead to loss of the whole forebrain in mice. If EXOSC10 is lost a few days later (from E10.5), the forebrain develops, however, the cortical size is reduced severely.

We found that EXOSC10 regulates the expression of apoptosis regulating factors by degrading their mRNAs. The binding of EXOSC10 to the transcripts of the P53 signaling mediators AEN and BBC3 and probably others leads to their rapid turnover. Loss of EXOSC10 creates an imbalance in production and decay of apoptosis-related transcripts leading to an accumulation of these, ultimately causing healthy cells in the developing cortex to undergo apoptosis. The resulting cell death contributes to the reduction of cortical size. As microcephaly is also observed in humans with an EXOSC10 mutation, one could assume that this function in the regulation of apoptosis is preserved from mouse to man.

Similar observations were done in a study of EXOSC8 or EXOSC9 mutant zebrafish (Muller et al., 2020). Zebrafish larvae with a homozygous frameshift mutation in EXOSC8 or EXOSC9 displayed smaller heads and eyes and cerebellar atrophy due to an increase of apoptotic cells (Muller et al., 2020). Consistently, higher P53 levels were found in affected zebrafish. Increased P53 was also observed in cultured human cells treated with siRNA for 
EXOSC8 or EXOSC9 mRNA as well as in muscle samples from patients with EXOSC9 mutations (Muller et al., 2020).

Thus, the role of the exosome complex in cell survival by regulating P53 signaling might be a general mechanism contributing to the neurodegeneration observed in human patients with mutations in exosome complex subunits. Nevertheless, the variability of human disorders caused by mutations of different subunits indicates that different pathways may be involved. The different subunits may have diverse cell type- and tissue-specific functions, which still need to be uncovered.

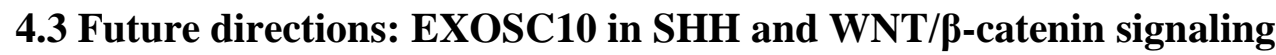

We are preparing a manuscript about EXOSC10 mutations causing microcephaly in patients (Fig. 1.1). The smaller brain corresponds to our finding that loss of EXOSC10 increases P53 levels, ultimately resulting in apoptosis. As all known patients are heterozygous for a mutation of EXOSC10, we decided to investigate the heterozygous condition in mice as well. Our first data show that the heterozygous brain is less severely affected than the homozygous conditional knockout, however, an increase of apoptotic cells and a reduced size of the cortex were detected corresponding to the microcephaly observed in humans with EXOSC10 mutation (Fig. 4.1A, B).

As no patients with a homozygous mutation of EXOSC10 are known, the homozygous condition is likely to be lethal, similar as in the EXOSC10cKO_FoxG1-Cre mice. In the FoxG1Cre mice, which loose EXOSC10 early starting from E8.5 (Hebert and McConnell, 2000), the forebrain fails to develop (Figure S3.2).

Nevertheless, regulating cell survival might not be the only way in which the exosome complex contributes to proper neurodevelopment. In EXOSC10cKO_Emxl-Cre mouse embryos, a decrease of neural stem cells and an increase of intermediate progenitor cells and neuronal cells was observed (Fig. 4.1C, D). These observations correspond to previous studies reporting that the exosome complex regulates the balance of differentiation and progenitor maintenance in other cell types, such as embryonic stem cells, epidermal cells and erythroid precursor cells (Belair et al., 2019; McIver et al., 2014; McIver et al., 2016; Mistry et al., 2012).

RNA sequencing of E12.5 EXOSC10 cKO mouse cortices revealed an upregulation of neuron differentiation transcripts combined with downregulation of transcripts of genes known to be involved in NSC fate and cell cycle (Fig. 4.1E, F). Interestingly, several transcripts of SHH signaling ( $\mathrm{SHH}, \mathrm{PTCH}$ ) and WNT signaling pathways (WNT3, WNT9b) were upregulated.

Binding of SHH to Patched (PTCH) receptors (PTCH1 and PTCH2) relieves the PTCHmediated inhibition of the Smoothened (SMO) receptor (reviewed by Choudhry et al., 2014; Rimkus et al., 2016). The resulting signal transduction from SMO through the cytoplasmic SUFU-GLI complex leads to the activation of GLI1-3. GLI1-3 are transcription factors modulating the expression of target genes with GLI1 and GLI2 acting as transcriptional activators and GLI3 as repressor. 
Binding of WNT activators (e.g. WNT3) to the Frizzled/LRP receptor complex leads to the release of $\beta$-catenin from the cytoplasmic $\beta$-catenin destruction complex (reviewed by Logan and Nusse, 2004; MacDonald et al., 2009). $\beta$-catenin, which is degraded in the absence of WNT, then accumulates in the nucleus where it acts as a transcription factor.

Crosstalk between the SHH and WNT pathways forms regulatory loops (Ding and Wang, 2017; Mullor et al., 2001; Nakamura et al., 2013; Noubissi et al., 2009; Song et al., 2015). For example, GLI2/3 was found to regulate WNT proteins (Mullor et al., 2001). Additionally, SHH was shown to increase $\beta$-catenin levels through the transcription factor $\mathrm{N}$ myc in cerebellar granule neuron progenitors (Mani et al., 2020). Vice versa, WNT/ $\beta$-catenin signaling was found to increase GLI1 expression by inducing expression of the RNA-binding protein CRD-BP, which binds and stabilizes GLI1 transcripts (Noubissi et al., 2009).

In patterning of the CNS, SHH and WNT signaling are known to have opposing effects. Whereas SHH induces ventral identity, WNT signaling is responsible for the dorsal fate (Ulloa and Marti, 2010). This results in the production of pyramidal neurons in the dorsal part and interneurons in the ventral part of the telencephalon, creating the two main populations of cortical neurons (Gulacsi and Anderson, 2008; Li et al., 2009).

In the context of balancing proliferation and differentiation, both, SHH and WNT signaling, have been shown to be essential for progenitor proliferation as well neuronal differentiation. Several studies showed that SHH signaling promotes proliferation in the developing brain. In SHH null mice, not only dorsoventral patterning but also general brain proliferation is affected leading to a severe reduction in size (Chiang et al., 1996; Rallu et al., 2002). Ectopic SHH expression in the developing spinal cord revealed an essential function in the expansion of granule cell precursors (Rowitch et al., 1999). Also in the postnatal telencephalon, SHH was shown to be required to maintain progenitor cells (Machold et al., 2003). In addition to its role in NSCs, SHH signaling was also shown to be important for neuronal differentiation. In the dorsal telencephalon, upregulation of SHH signaling increased the transition of RGCs to IPCs (Shikata et al., 2011). Studies indicated that SHH regulates proliferation and differentiation of cortical neurons through coordination of cell cycle kinetics (Komada et al., 2008; Ruiz i Altaba et al., 2002). In conclusion, the various roles of SHH signaling during development of the telencephalon depend on the region, concentration and timing of expression (Komada, 2012).

Several studies revealed that canonical WNT signaling promotes proliferation. Overexpression of $\beta$-catenin increased cortical NSC proliferation (Chenn and Walsh, 2002). Also the expression of neurogenic genes was delayed by ectopic expression of $\beta$-catenin and accelerated by $\beta$-catenin ablation (Machon et al., 2007). Nevertheless, the canonical WNT pathway also regulates the differentiation of IPC into neurons. Overexpression of the WNT activator WNT3a leads to upregulation the WNT signaling and induces premature differentiation of IPC into neurons (Munji et al., 2011). The authors suggest that the effects of WNT signaling depend on the context defined by the progenitor state and/or the developmental time (Munji et al., 2011). This idea is further supported by a study indicating that canonical WNT signaling affects the fate-switch of progenitors and controls the stage-specific production of progenitors and neuronal subtypes (Draganova et al., 2015). 
Our preliminary data suggest that dysregulation of SHH and WNT signaling is likely to contribute to the phenotype of EXOSC10cKO mice. The role of EXOSC10 in the complex interplay of canonical SHH/WNT signaling in cortical patterning and in the proliferation/differentiation balance will be subject of our future investigations.

Besides, in human diseases, mutations of different subunits cause distinct phenotypes with different tissues and cell types affected. Similarly, the subunits display different expression patterns in the developing cortex (Fig S3.1). Hence, one could assume tissue- and cell typespecific functions of the subunits. Additionally, yet unknown interactions with other proteins could confer the tissue specificity. Therefore, further studies on the role of the exosome complex and its individual subunits in different tissues are necessary. For example, it would be interesting to use different mouse models for the individual complex subunits of the exosome complex to investigate their probably different roles in cortical development. Further molecular approaches, like co-immunoprecipitation, could reveal specific interactions of the individual subunits with other regulatory factors. 
A

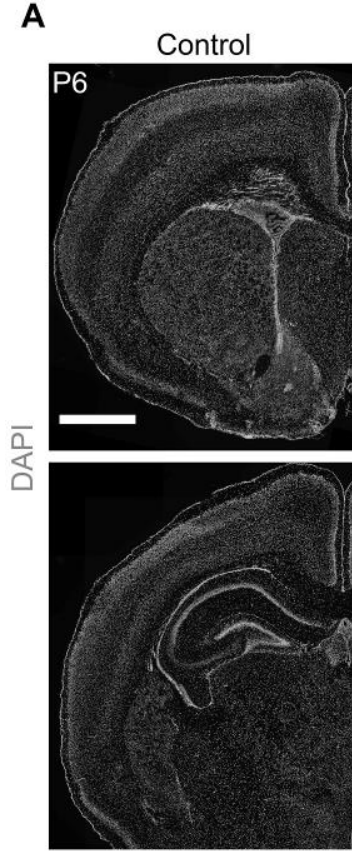

C

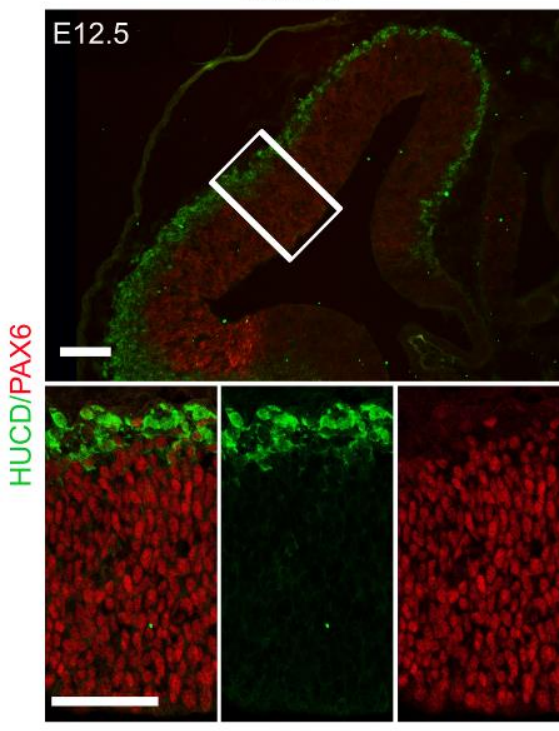

D
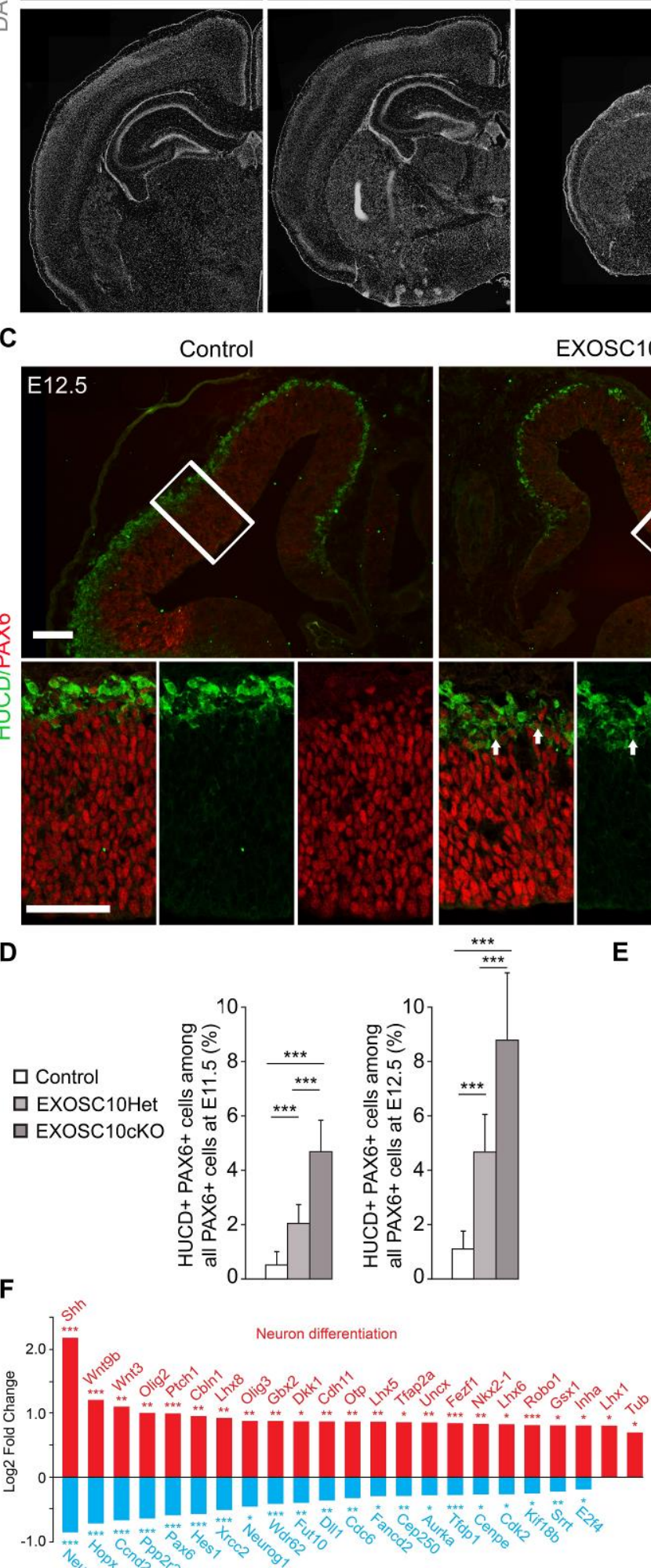

E
EXOSC10cKO

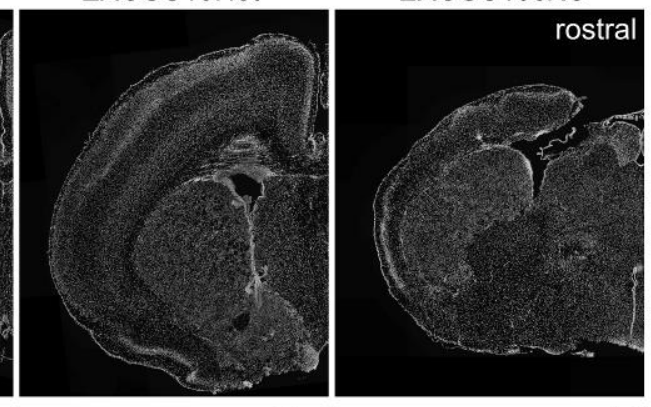

caudal

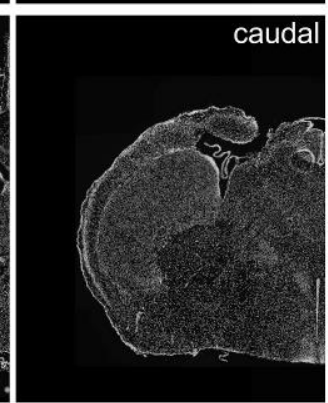

EXOSC10Het
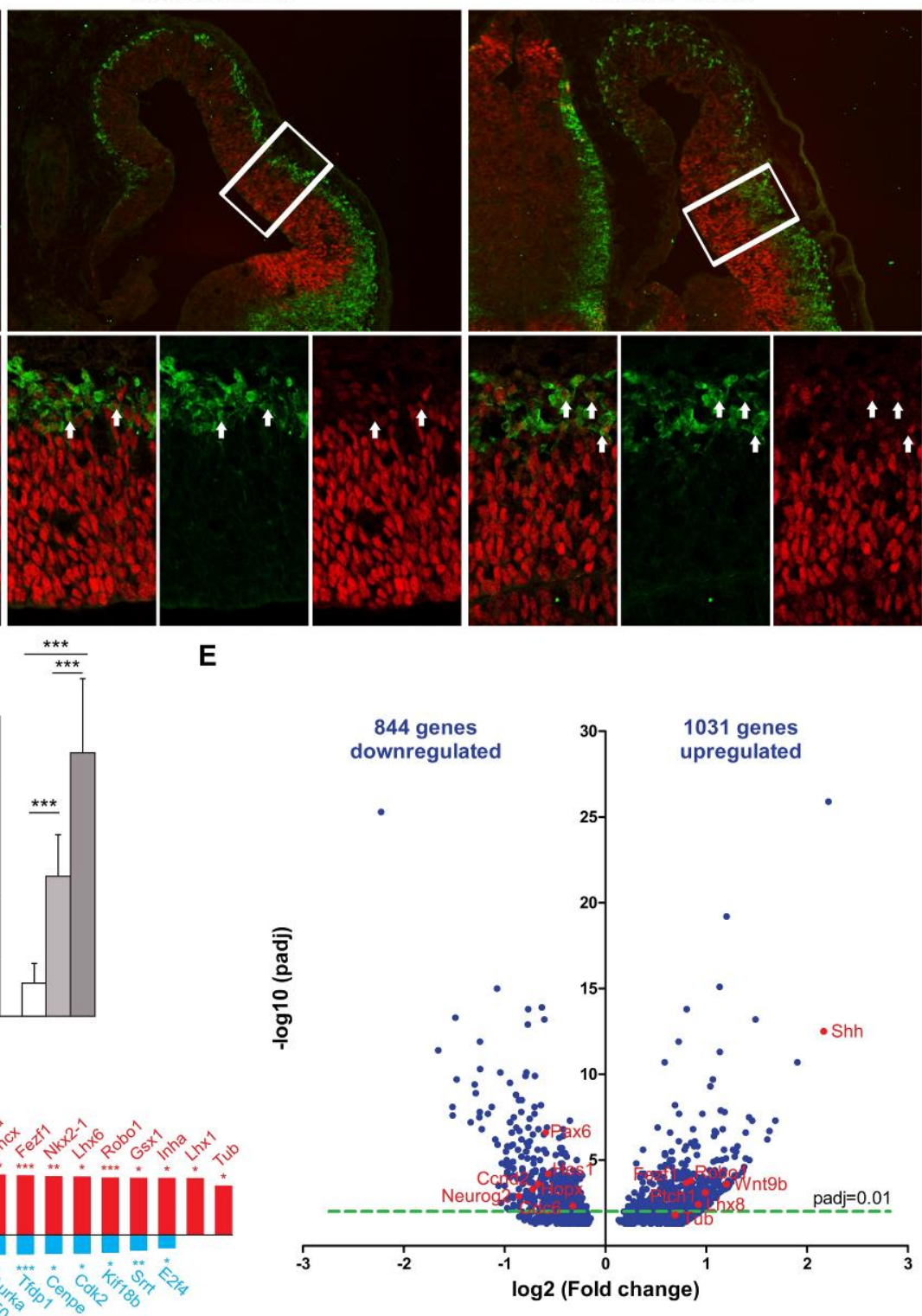

$\square$ Control

$\square$ EXOSC $10 \mathrm{Het}$

$\square$ EXOSC10cKO

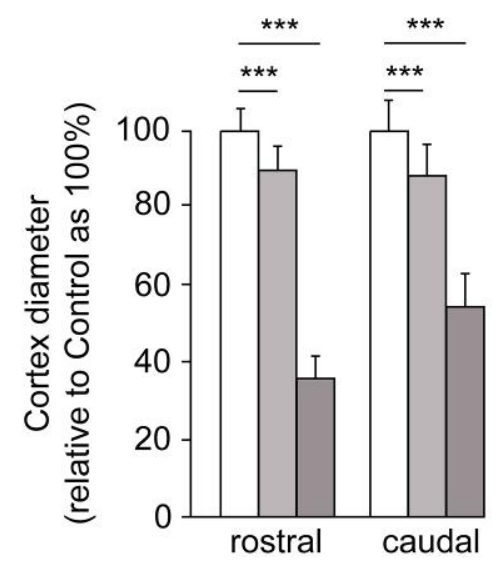

EXOSC10cKO

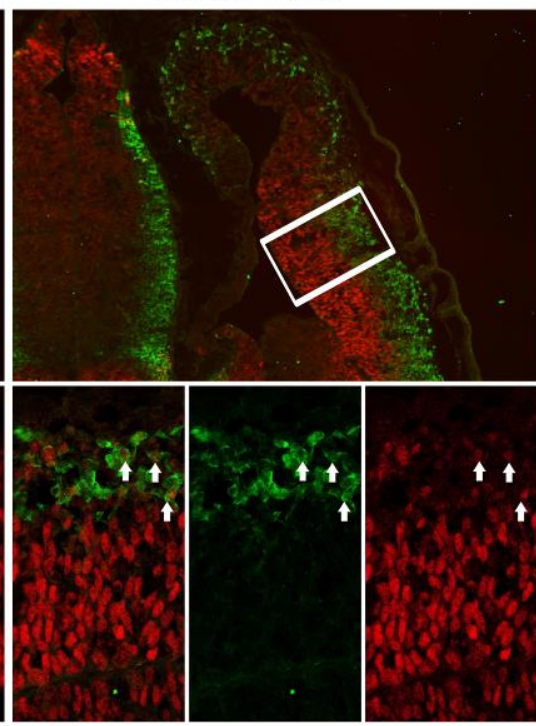

$1+$

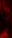

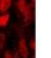


Figure 4.1 Loss of EXOSC10 leads to reduction of cortical size and promotes neuronal differentiation in homozygous and heterozygous condition.

(A) DAPI staining of P6 control (wild-type), EXOSC10Het (EXOSC10 COIN/+ Emxl-Cre) and EXOSC10cKO

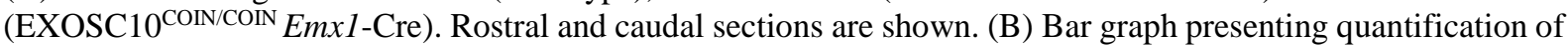
the cortical diameter in P6 EXOSC10Het and cKO mice relative to control displaying significant differences. (C) Micrographs at 10x and 40x magnifications of E12.5 control, EXOSC10Het and cKO cortex immunostained for PAX6 and HUCD. Lower images are higher magnifications indicated by the white frame. (D) Bar graph showing quantification of cells positive for PAX6 AND HUCD among all PAX6+ cells in the E12.5 control, EXOSC10Het and $\mathrm{cKO}$ in the cortical area marked with a white frame in C. (E) Volcano plot showing changes in gene expression detected in RNA-seq (E12.5 EXOSC10cKO cortex versus control) (for method see 3.5.4). (F) Bar graph showing upregulated (enriched) neuron differentiation genes and downregulated NSC and cell cycle genes in EXOSC10cKO cortex compared to control at E12.5 (revealed by RNA-seq). Statistical analyses were carried out using Student's t-test. Graphs are plotted as mean $( \pm \mathrm{SEM})$. An unpaired t-test was carried out on the average from at least two biological replicates for each condition. $* P<0.05, * * P<0.01, * * * P<0.005$. Scale bars: $1 \mathrm{~mm}(\mathrm{~A}), 100$ $\mu \mathrm{m}(\mathrm{C})$.

\subsection{RNA editing gives hope for future therapies}

The increasing number of neurodevelopmental diseases linked to mutations in genes encoding subunits of the exosome complex emphasizes the importance of stepping up research efforts into understanding how the exosome complex regulates neurodevelopment. So far, for exosome complex related diseases (summarized in Table 1, page 12), no cure is available and only symptoms can be treated. In future, the novel technique of RNA editing might be the treatment of choice and improve the lives of affected individuals.

In the last few years, genome editing using the CRISPR system became a promising research area to develop new therapies for genetic diseases (Wu et al., 2020). However, possible "off-target" effects could introduce unwanted changes, which would be permanent in the genome (Fu et al., 2013; Zhang et al., 2015). RNA editing might be a reasonable alternative enabling to change the genetic output without the need of genome editing (Christofi and Zaravinos, 2019; Reardon, 2020). As mRNAs are stable only for a limited time, possible introduced errors would be removed quickly, making this therapy reversible and less dangerous than genome editing (Merkle et al., 2019). Besides, with RNA editing it is possible to introduce temporary changes making the method also interesting for the treatment of various other diseases (Katrekar et al., 2019; Merkle et al., 2019; Reardon, 2020). For example, turning off key signaling factors temporarily could be a powerful tool in the treatment of tumours (Merkle et al., 2019; Reardon, 2020).

The most common type of RNA editing, is the A-to-I conversion, which means the change of adenosine to inosine (Bazak et al., 2014). During translation, ribosomes read inosine as the familiar guanosine (Basilio et al., 1962). The enzymes, which bind to RNA and perform the A-to-I conversion, are called ADARs (adenosine deaminases acting on RNA) (Bass, 2002; Nishikura, 2016). In mammals, three ADAR genes (ADAR1-3) were found to generate four isoforms DAR1p150, ADAR1p110, ADAR2 and ADAR3 (Melcher et al., 1996a; Melcher et al., 1996b). Another group of enzymes belong to the AID/APOBEC (activation-induced cytidine deaminase/apolipoprotein B editing complex) family and perform a C-to-U conversion meaning they change cytidine to uridine in mRNA (Fossat et al., 2014; Smith et al., 2012). 
Different approaches are used to direct ADARs to target mRNA. Sequence-specific guide RNA can either be attached to ADAR or contain an ADAR-recruitment domain (Fukuda et al., 2017; Katrekar et al., 2019; Merkle et al., 2019; Montiel-Gonzalez et al., 2013; Vogel et al., 2018). By attaching ADARs to guide RNA, which binds to a specific stretch of mRNA creating a double-strand, ADARs can be engineered to alter a specific mRNA (Montiel-Gonzalez et al., 2013; Vogel et al., 2018). Using this, defective mRNA was successfully edited in Xenopus embryos as well as in cultured human embryonic kidney cells (Montiel-Gonzalez et al., 2013). In a mouse model for Duchenne muscular dystrophy caused by a mutation inducing a premature stop codon in the dystrophin gene, injection of a guide RNA containing ADAR-recruiting domains packed in an AAV vector into muscle tissue could restore dystrophin expression (Katrekar et al., 2019).

Alternatively, an ADAR can also be fused to an RNA-targeting protein like CAS13 to direct the ADAR to certain transcripts specifically (Abudayyeh et al., 2019; Cox et al., 2017).

Despite the promising findings, there are several limitations to this novel technique, which is just starting to evolve. One challenge is the efficiency of RNA editing, as ADARs are less efficient than CRISPR (Reardon, 2020). Additionally, they might have to compete with nonsense mediated decay of their target mRNA (Katrekar et al., 2019).

Using ADARs or AID/APOBECs to edit RNA, it is possible to change a single nucleotide. By this, a pathogenic base substitution could be reversed. However, if the disease causing allele contains a nucleotide deletion or inversion, the resulting mRNA cannot be corrected by the known RNA editing tools.

Besides, even the ability to exchange single nucleotides is restricted to changes from adenosine to guanine and from cytosine to uracil further limiting the disease variants, which could be treated. Current RNA editing possibilities could be expanded not only by finding more RNA editing enzymes in nature but also by altering existing enzymes. For example, by changing its sequence, ADAR2 was evolved to convert C to U (Abudayyeh et al., 2019). The engineered cytidine deaminase is still able to convert A to I as well (Abudayyeh et al., 2019). The function of the modified ADAR2 enzyme was tested successfully in cultured human cells (Abudayyeh et al., 2019).

Another challenge is the transfer of the engineered guide mRNA into the cells. Chemical modifications could stabilize guide RNAs to prevent degradation or they could be transferred into cells via nanoparticles or viruses (Merkle et al., 2019; Reardon, 2020).

ADARs occur naturally in human cells, however in most tissues they are produced only in small amounts raising the question if relying on the naturally occurring ADARs is sufficient or if it might be necessary to add ADARs (Merkle et al., 2019; Reardon, 2020). Also attracting natural occurring ADARs via ADAR-recruiting domains in guide mRNA to the target mRNA could lower their capability to fulfil their normal functions (Katrekar et al., 2019; Reardon, 2020). This could lead to so far unknown health issues and it shows that more research is needed before the technique can be applied in humans.

Once these challenges are overcome, the diseases caused by mutations in the exosome complex are obvious candidates for a treatment with RNA editing as the diseases are caused by 
a base substitution in most of the individuals. Modifying the mRNA of the affected exosome complex subunit could reverse the effect of the genetic mutation at least partially.

One challenge in the treatment of diseases caused by mutations of the exosome complex would be the starting point of treatment. Diagnosis usually occurs when symptoms are already present. At this time point, processes like apoptosis may already have caused damage, which cannot be reversed completely. However, as brain growth and development continues, starting a treatment in the first years of life would still have potential to reduce the severity of symptoms. Especially, with PCH1 and HSP being progressive neurodegenerative diseases, they are good candidates for intervention.

\subsection{EXOSC10 might be beneficial for a novel RNA editing tool}

In addition to the RNA editing tools mentioned before, others tools have been used to target defective m6A sites (reviewed by Sokpor et al., 2021). Therefore, the ability of CAS13 family proteins to target RNA is used (Burmistrz et al., 2020; Wang et al., 2019). Deactivated CAS13b (dCAS18b) is catalytically inactive, but its RNA-binding ability is preserved. A guide RNA (gRNA) is fused to dCAS13b to target a specific RNA sequence. The gRNA-dCAS13b is further coupled to a certain effector. This effector can be an m6A writer to add an m6A methyl group, an $\mathrm{m} 6 \mathrm{~A}$ eraser to demethylate $\mathrm{m} 6 \mathrm{~A}$ or $\mathrm{m} 6 \mathrm{~A}$ readers to enhance translation or induce RNA degradation (Sokpor et al., 2021). Recently, several transcripts have been successfully targeted by this tool (Li et al., 2020a; Mo et al., 2020; Rauch et al., 2018; Wilson et al., 2020; Zhao et al., 2020).

For instance, malfunction of methyltransferases (METTL3 and/or METTL14) leads to missing m6A marks on gene transcripts (Agarwala et al., 2012; Geula et al., 2015; Liu et al., 2014). Fusions of dCAS13 with a METTL3 methyltransferase domain or with a modified METTL3:METTL14 complex can compensate this malfunction by introducing m6A marks (Wilson et al., 2020). The site-specific addition of methylation marks was shown for several transcripts, including the ones from SOX2, FOXM1 and ZNF638 in human embryonic kidney cells (Wilson et al., 2020).

When used as an effector fused to dCAS13b, the m6A reader YTHDF2 was reported to induce degradation of the targeted transcript (Rauch et al., 2018). However, the degradation is limited to transcripts known to be m6A modified. By using EXOSC10 as the effector, the possibilities of RNA editing could be further expanded. A gRNA-dCAS18b-EXOSC10 complex could degrade various target mRNAs specifically and directly without the need of m6A marks.

The resulting new tool for targeted RNA degradation could be applied for the treatment of various genetic diseases. Especially, gain-of-function mutations, which lead to increased abundance or activity of a certain protein, could be treated by this tool. Binding to a specific mRNA by dCAS18b followed by EXOSC10-mediated degradation could reduce the amount of mRNA resulting in decreased amount of protein. Notably, rather than knocking out a specific gene, the degradation of its transcript would be dose-dependent and reversible.

For example, gain-of-function mutations in the RARB gene cause a complex neurological phenotype (Srour et al., 2016; Srour et al., 2013). Affected patients display eye 
malformations (microphthalmia) and severe developmental delay with progressive motor impairment (Srour et al., 2016). RARB stands for retinoic acid receptor beta, one subtype of several existing retinoic acid (RA) receptors. Upon binding of RA, the RA receptor complex acts as a transcription factor by binding to specific DNA sequences, known as RA response elements (RAREs) (Collins, 2002; Glass and Rosenfeld, 2000). The pathogenic mutations of RARB identified in patients increase its transcriptional activation potential in response to RA ligands by two- to threefold (Srour et al., 2016; Srour et al., 2013). EXOSC10-mediated degradation of a certain proportion of RARB transcripts could counteract the increased activity of the RARB protein. An early intervention has potential to improve cognitive and motor development of affected patients.

Similarly, diseases caused by gene duplications could potentially treated by RNA editing using EXOSC10. For example, duplications and triplications of the gene coding for $\alpha-$ synuclein (SNCA) have been linked to familiar Parkinson's disease (Nishioka et al., 2006; Singleton et al., 2003). Targeted degradation of SNCA transcripts by EXOSC10 could change the amount of SNCA protein back to physiological levels.

So far, a gRNA-CAS18b-EXOSC10 complex has not been created and the functionality of such a complex would have to be tested in cells at first. Nevertheless, the use of EXOSC10 for RNA editing might be a reasonable and promising research area to add to existing RNA editing tools.

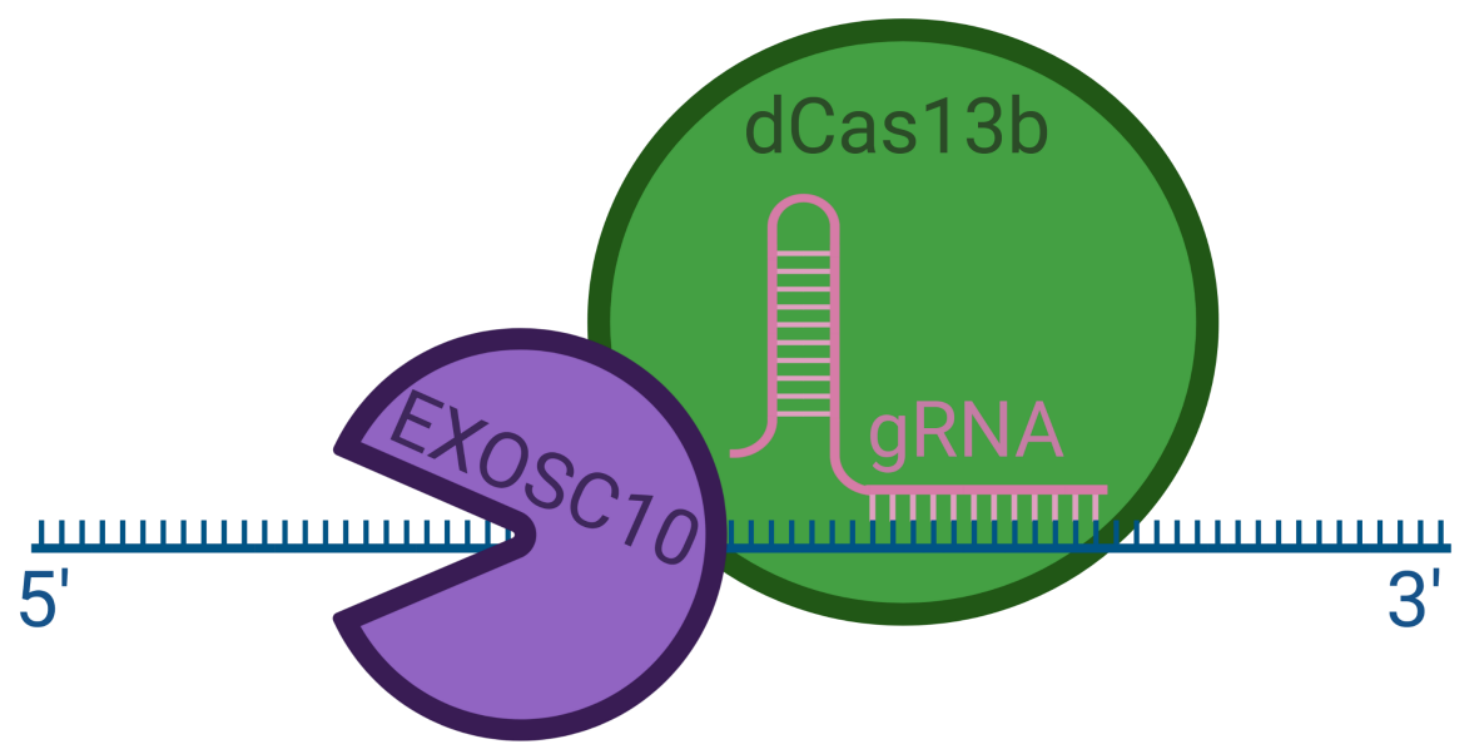

Figure 4.2 Illustration of a proposed gRNA-dCAS18b-EXOSC10 complex. Deactivated Cas13b (dCas13b) is fused with a guide RNA (gRNA) to specifically target a certain mRNA. Coupling to EXOSC10 may lead to degradation of the targeted transcript. Created with BioRender.com. 


\section{Summary}

This work focuses on elucidating the molecular mechanisms that control the cortical development. Identification of genes and factors that direct the development of the cerebral cortex will both tell us about their disease-related importance and improve our understanding of the normal formation and malformation of the cortex.

In the first part, we performed transcriptome analysis to determine the molecular profile of IPCs, which play a crucial role in cortical formation as they generate the majority of cortical neurons. Accordingly, we sorted TBR2+ IPCs from the embryonic mouse cortex and analysed gene expression profiles of TBR2+ IPCs versus TBR2- cell populations. We identified different levels of key genetic factors regulating chromatid segregation, cell-cycle progression, transcription, and cell signaling. Remarkably, in humans, mutations of several identified IPC genes are linked to various cortical malformations, like microcephaly and macrocephaly, corpus callosum defects, and neurological deficits. For example, mutations in the cohesin acetyltransferase ESCO2, one of the newly identified IPC genes, cause severe malformations including microcephaly. We showed that deficiency of ESCO2 in the developing mouse cortex leads to severe loss of IPCs, resulting in cortical malformation. We thereby demonstrate the identification of a central genetic factor of IPC genesis. Our molecular profiling data reveal novel molecular characteristics of IPCs and offer a resource for future investigations.

Recent sequencing analyses of cortical malformations revealed a multifarious genetic landscape. In our pilot work, we identified novel microcephaly-related mutations in a gene encoding EXOSC10, a core subunit of the RNA-decay exosome complex. In the second part of this work, we characterized the cortical phenotypes of EXOSC10cKO mutants. We showed that EXOSC10 is essential for forebrain formation. EXOSC10 deficiency in the developing mouse cortex causes massive apoptosis in cortical cells resulting in cortical malformation. We found that EXOSC10 binds and degrades mRNA coding for P53 signaling-mediators, like AEN and BBC3. Additionally, our studies indicate that EXOSC10 plays a role in regulating the differentiation of cortical progenitors. It might do so via degrading transcripts of the SHH/WNT- $\beta$ catenin signaling pathways. Further investigations are needed to illuminate this additional role of EXOSC10. In conclusion, our study reveals an essential role of EXOSC10 in suppressing the P53, SHH/WNT- $\beta$ catenin pathways, which are indispensable for cell survival, neurogenesis and normal cortical formation. Our findings of the mouse model correspond to observations of humans with microcephaly linked to EXOSC10 mutations. 


\section{References}

Abdollahi, M.R., Morrison, E., Sirey, T., Molnar, Z., Hayward, B.E., Carr, I.M., Springell, K., Woods, C.G., Ahmed, M., Hattingh, L., et al. (2009). Mutation of the variant alpha-tubulin TUBA8 results in polymicrogyria with optic nerve hypoplasia. Am J Hum Genet 85, 737744.

Abudayyeh, O.O., Gootenberg, J.S., Franklin, B., Koob, J., Kellner, M.J., Ladha, A., Joung, J., Kirchgatterer, P., Cox, D.B.T., and Zhang, F. (2019). A cytosine deaminase for programmable single-base RNA editing. Science 365, 382-386.

Afgan, E., Baker, D., Batut, B., van den Beek, M., Bouvier, D., Cech, M., Chilton, J., Clements, D., Coraor, N., Gruning, B.A., et al. (2018). The Galaxy platform for accessible, reproducible and collaborative biomedical analyses: 2018 update. Nucleic Acids Res 46, W537-W544.

Agarwala, S.D., Blitzblau, H.G., Hochwagen, A., and Fink, G.R. (2012). RNA methylation by the MIS complex regulates a cell fate decision in yeast. PLoS Genet 8, e1002732.

Agirman, G., Broix, L., and Nguyen, L. (2017). Cerebral cortex development: an outside-in perspective. FEBS Lett 591, 3978-3992.

Agoston, Z., Li, N., Haslinger, A., Wizenmann, A., and Schulte, D. (2012). Genetic and physical interaction of Meis2, Pax3 and Pax7 during dorsal midbrain development. BMC Dev Biol 12, 10.

Albert, M., Kalebic, N., Florio, M., Lakshmanaperumal, N., Haffner, C., Brandl, H., Henry, I., and Huttner, W.B. (2017). Epigenome profiling and editing of neocortical progenitor cells during development. EMBO J 36, 2642-2658.

Albig, A.R., and Decker, C.J. (2001). The target of rapamycin signaling pathway regulates mRNA turnover in the yeast Saccharomyces cerevisiae. Mol Biol Cell 12, 3428-3438.

Alcamo, E.A., Chirivella, L., Dautzenberg, M., Dobreva, G., Farinas, I., Grosschedl, R., and McConnell, S.K. (2008). Satb2 regulates callosal projection neuron identity in the developing cerebral cortex. Neuron 57, 364-377.

Alkuraya, F.S., Cai, X., Emery, C., Mochida, G.H., Al-Dosari, M.S., Felie, J.M., Hill, R.S., Barry, B.J., Partlow, J.N., Gascon, G.G., et al. (2011). Human mutations in NDE1 cause extreme microcephaly with lissencephaly [corrected]. Am J Hum Genet 88, 536-547.

Allmang, C., Petfalski, E., Podtelejnikov, A., Mann, M., Tollervey, D., and Mitchell, P. (1999). The yeast exosome and human PM-Scl are related complexes of 3' --> 5' exonucleases. Genes Dev 13, 2148-2158.

Aloy, P., Ciccarelli, F.D., Leutwein, C., Gavin, A.C., Superti-Furga, G., Bork, P., Bottcher, B., and Russell, R.B. (2002). A complex prediction: three-dimensional model of the yeast exosome. EMBO Rep 3, 628-635.

Amamoto, R., Zuccaro, E., Curry, N.C., Khurana, S., Chen, H.H., Cepko, C.L., and Arlotta, P. (2020). FIN-Seq: transcriptional profiling of specific cell types from frozen archived tissue of the human central nervous system. Nucleic Acids Res 48, e4.

Anderson, J.R., Mukherjee, D., Muthukumaraswamy, K., Moraes, K.C., Wilusz, C.J., and Wilusz, J. (2006). Sequence-specific RNA binding mediated by the RNase PH domain of components of the exosome. RNA 12, 1810-1816.

Anderson, J.S., and Parker, R.P. (1998). The 3' to 5' degradation of yeast mRNAs is a general mechanism for mRNA turnover that requires the SKI2 DEVH box protein and $3^{\prime}$ to $5^{\prime}$ exonucleases of the exosome complex. EMBO J 17, 1497-1506.

Arlotta, P., Molyneaux, B.J., Chen, J., Inoue, J., Kominami, R., and Macklis, J.D. (2005). Neuronal subtype-specific genes that control corticospinal motor neuron development in vivo. Neuron 45, 207-221. 
Arnold, S.J., Huang, G.J., Cheung, A.F., Era, T., Nishikawa, S., Bikoff, E.K., Molnar, Z., Robertson, E.J., and Groszer, M. (2008). The T-box transcription factor Eomes/Tbr2 regulates neurogenesis in the cortical subventricular zone. Genes Dev 22, 2479-2484.

Baala, L., Briault, S., Etchevers, H.C., Laumonnier, F., Natiq, A., Amiel, J., Boddaert, N., Picard, C., Sbiti, A., Asermouh, A., et al. (2007). Homozygous silencing of T-box transcription factor EOMES leads to microcephaly with polymicrogyria and corpus callosum agenesis. Nat Genet 39, 454-456.

Bachmann, C., Nguyen, H., Rosenbusch, J., Pham, L., Rabe, T., Patwa, M., Sokpor, G., Seong, R.H., Ashery-Padan, R., Mansouri, A., et al. (2016). mSWI/SNF (BAF) Complexes Are Indispensable for the Neurogenesis and Development of Embryonic Olfactory Epithelium. PLoS Genet 12, e1006274.

Bahi-Buisson, N., Poirier, K., Fourniol, F., Saillour, Y., Valence, S., Lebrun, N., Hully, M., Bianco, C.F., Boddaert, N., Elie, C., et al. (2014). The wide spectrum of tubulinopathies: what are the key features for the diagnosis? Brain 137, 1676-1700.

Bakircioglu, M., Carvalho, O.P., Khurshid, M., Cox, J.J., Tuysuz, B., Barak, T., Yilmaz, S., Caglayan, O., Dincer, A., Nicholas, A.K., et al. (2011). The essential role of centrosomal NDE1 in human cerebral cortex neurogenesis. Am J Hum Genet 88, 523-535.

Barkovich, A.J., Guerrini, R., Kuzniecky, R.I., Jackson, G.D., and Dobyns, W.B. (2012). A developmental and genetic classification for malformations of cortical development: update 2012. Brain 135, 1348-1369.

Basilio, C., Wahba, A.J., Lengyel, P., Speyer, J.F., and Ochoa, S. (1962). Synthetic polynucleotides and the amino acid code. V. Proc Natl Acad Sci U S A 48, 613-616.

Bass, B.L. (2002). RNA editing by adenosine deaminases that act on RNA. Annu Rev Biochem 71, 817-846.

Bazak, L., Haviv, A., Barak, M., Jacob-Hirsch, J., Deng, P., Zhang, R., Isaacs, F.J., Rechavi, G., Li, J.B., Eisenberg, E., et al. (2014). A-to-I RNA editing occurs at over a hundred million genomic sites, located in a majority of human genes. Genome Res 24, 365-376.

Belair, C., Sim, S., Kim, K.Y., Tanaka, Y., Park, I.H., and, and Wolin, S.L. (2019). The RNA exosome nuclease complex regulates human embryonic stem cell differentiation. J Cell Biol $218,2564-2582$.

Bellows, A.M., Kenna, M.A., Cassimeris, L., and Skibbens, R.V. (2003). Human EFO1p exhibits acetyltransferase activity and is a unique combination of linker histone and Ctf7p/Eco1p chromatid cohesion establishment domains. Nucleic Acids Res 31, 6334-6343.

Betizeau, M., Cortay, V., Patti, D., Pfister, S., Gautier, E., Bellemin-Menard, A., Afanassieff, M., Huissoud, C., Douglas, R.J., Kennedy, H., et al. (2013). Precursor diversity and complexity of lineage relationships in the outer subventricular zone of the primate. Neuron 80, 442-457.

Biancheri, R., Cassandrini, D., Pinto, F., Trovato, R., Di Rocco, M., Mirabelli-Badenier, M., Pedemonte, M., Panicucci, C., Trucks, H., Sander, T., et al. (2013). EXOSC3 mutations in isolated cerebellar hypoplasia and spinal anterior horn involvement. J Neurol 260, 18661870.

Bilguvar, K., Ozturk, A.K., Louvi, A., Kwan, K.Y., Choi, M., Tatli, B., Yalnizoglu, D., Tuysuz, B., Caglayan, A.O., Gokben, S., et al. (2010). Whole-exome sequencing identifies recessive WDR62 mutations in severe brain malformations. Nature 467, 207-210.

Bizzari, S., Hamzeh, A.R., Mohamed, M., Al-Ali, M.T., and Bastaki, F. (2020). Expanded PCH1D phenotype linked to EXOSC9 mutation. Eur J Med Genet 63, 103622.

Bizzotto, S., and Francis, F. (2015). Morphological and functional aspects of progenitors perturbed in cortical malformations. Front Cell Neurosci 9, 30.

Blanchart, A., Navis, A.C., Assaife-Lopes, N., Usoskin, D., Aranda, S., Sontheimer, J., and Ernfors, P. (2018). UHRF1 Licensed Self-Renewal of Active Adult Neural Stem Cells. Stem Cells 36, 1736-1751. 
Blaschke, A.J., Staley, K., and Chun, J. (1996). Widespread programmed cell death in proliferative and postmitotic regions of the fetal cerebral cortex. Development 122, 11651174.

Blomen, V.A., Majek, P., Jae, L.T., Bigenzahn, J.W., Nieuwenhuis, J., Staring, J., Sacco, R., van Diemen, F.R., Olk, N., Stukalov, A., et al. (2015). Gene essentiality and synthetic lethality in haploid human cells. Science 350, 1092-1096.

Boccaletto, P., Machnicka, M.A., Purta, E., Piatkowski, P., Baginski, B., Wirecki, T.K., de Crecy-Lagard, V., Ross, R., Limbach, P.A., Kotter, A., et al. (2018). MODOMICS: a database of RNA modification pathways. 2017 update. Nucleic Acids Res 46, D303-D307.

Boczonadi, V., Muller, J.S., Pyle, A., Munkley, J., Dor, T., Quartararo, J., Ferrero, I., Karcagi, V., Giunta, M., Polvikoski, T., et al. (2014). EXOSC8 mutations alter mRNA metabolism and cause hypomyelination with spinal muscular atrophy and cerebellar hypoplasia. Nat Commun 5, 4287.

Bohley, P., and Seglen, P.O. (1992). Proteases and proteolysis in the lysosome. Experientia 48, 151-157.

Bolcun-Filas, E., Bannister, L.A., Barash, A., Schimenti, K.J., Hartford, S.A., Eppig, J.J., Handel, M.A., Shen, L., and Schimenti, J.C. (2011). A-MYB (MYBL1) transcription factor is a master regulator of male meiosis. Development 138, 3319-3330.

Boo, S.H., and Kim, Y.K. (2020). The emerging role of RNA modifications in the regulation of mRNA stability. Exp Mol Med 52, 400-408.

Boonsawat, P., Joset, P., Steindl, K., Oneda, B., Gogoll, L., Azzarello-Burri, S., Sheth, F., Datar, C., Verma, I.C., Puri, R.D., et al. (2019). Elucidation of the phenotypic spectrum and genetic landscape in primary and secondary microcephaly. Genet Med 21, 2043-2058.

Brands, A., and Skibbens, R.V. (2005). Ctf7p/Eco1p exhibits acetyltransferase activity--but does it matter? Curr Biol 15, R50-51.

Britanova, O., de Juan Romero, C., Cheung, A., Kwan, K.Y., Schwark, M., Gyorgy, A., Vogel, T., Akopov, S., Mitkovski, M., Agoston, D., et al. (2008). Satb2 is a postmitotic determinant for upper-layer neuron specification in the neocortex. Neuron 57, 378-392.

Brust-Mascher, I., and Scholey, J.M. (2002). Microtubule flux and sliding in mitotic spindles of Drosophila embryos. Mol Biol Cell 13, 3967-3975.

Burmistrz, M., Krakowski, K., and Krawczyk-Balska, A. (2020). RNA-Targeting CRISPR-Cas Systems and Their Applications. Int J Mol Sci 21.

Burns, D.T., Donkervoort, S., Muller, J.S., Knierim, E., Bharucha-Goebel, D., Faqeih, E.A., Bell, S.K., AlFaifi, A.Y., Monies, D., Millan, F., et al. (2018). Variants in EXOSC9 Disrupt the RNA Exosome and Result in Cerebellar Atrophy with Spinal Motor Neuronopathy. Am J Hum Genet 102, 858-873.

Butler, J.S., and Mitchell, P. (2011). Rrp6, rrp47 and cofactors of the nuclear exosome. Adv Exp Med Biol 702, 91-104.

Calegari, F., Haubensak, W., Haffner, C., and Huttner, W.B. (2005). Selective lengthening of the cell cycle in the neurogenic subpopulation of neural progenitor cells during mouse brain development. J Neurosci 25, 6533-6538.

Callis, J. (1995). Regulation of Protein Degradation. Plant Cell 7, 845-857.

Cao, L.G., and Wang, Y.L. (1990). Mechanism of the formation of contractile ring in dividing cultured animal cells. I. Recruitment of preexisting actin filaments into the cleavage furrow. J Cell Biol 110, 1089-1095.

Cardoso, C., Leventer, R.J., Dowling, J.J., Ward, H.L., Chung, J., Petras, K.S., Roseberry, J.A., Weiss, A.M., Das, S., Martin, C.L., et al. (2002). Clinical and molecular basis of classical lissencephaly: Mutations in the LIS1 gene (PAFAH1B1). Hum Mutat 19, 4-15.

Carneiro, T., Carvalho, C., Braga, J., Rino, J., Milligan, L., Tollervey, D., and Carmo-Fonseca, M. (2007). Depletion of the yeast nuclear exosome subunit Rrp6 results in accumulation of polyadenylated RNAs in a discrete domain within the nucleolus. Mol Cell Biol 27, 41574165 . 
Cassandri, M., Smirnov, A., Novelli, F., Pitolli, C., Agostini, M., Malewicz, M., Melino, G., and Raschella, G. (2017). Zinc-finger proteins in health and disease. Cell Death Discov 3, 17071.

Caviness, V.S., Jr., Goto, T., Tarui, T., Takahashi, T., Bhide, P.G., and Nowakowski, R.S. (2003). Cell output, cell cycle duration and neuronal specification: a model of integrated mechanisms of the neocortical proliferative process. Cereb Cortex 13, 592-598.

Chapman, M.A., Lawrence, M.S., Keats, J.J., Cibulskis, K., Sougnez, C., Schinzel, A.C., Harview, C.L., Brunet, J.P., Ahmann, G.J., Adli, M., et al. (2011). Initial genome sequencing and analysis of multiple myeloma. Nature 471, 467-472.

Chen, C.Y., Gherzi, R., Ong, S.E., Chan, E.L., Raijmakers, R., Pruijn, G.J., Stoecklin, G., Moroni, C., Mann, M., and Karin, M. (2001). AU binding proteins recruit the exosome to degrade ARE-containing mRNAs. Cell 107, 451-464.

Chen, J., Bardes, E.E., Aronow, B.J., and Jegga, A.G. (2009). ToppGene Suite for gene list enrichment analysis and candidate gene prioritization. Nucleic Acids Res 37, W305-311.

Chen, J., and Chen, X. (2018). MYBL2 Is Targeted by miR-143-3p and Regulates Breast Cancer Cell Proliferation and Apoptosis. Oncol Res 26, 913-922.

Chenn, A., and Walsh, C.A. (2002). Regulation of cerebral cortical size by control of cell cycle exit in neural precursors. Science 297, 365-369.

Cheung, T.T., Weston, M.K., and Wilson, M.J. (2017). Selection and evaluation of reference genes for analysis of mouse (Mus musculus) sex-dimorphic brain development. PeerJ 5, e2909.

Chiang, C., Litingtung, Y., Lee, E., Young, K.E., Corden, J.L., Westphal, H., and Beachy, P.A. (1996). Cyclopia and defective axial patterning in mice lacking Sonic hedgehog gene function. Nature 383, 407-413.

Choudhry, Z., Rikani, A.A., Choudhry, A.M., Tariq, S., Zakaria, F., Asghar, M.W., Sarfraz, M.K., Haider, K., Shafiq, A.A., and Mobassarah, N.J. (2014). Sonic hedgehog signalling pathway: a complex network. Ann Neurosci 21, 28-31.

Christofi, T., and Zaravinos, A. (2019). RNA editing in the forefront of epitranscriptomics and human health. J Transl Med 17, 319.

Chuang, S.M., Wang, Y., Wang, Q., Liu, K.M., and Shen, Q. (2011). Ebf2 marks early cortical neurogenesis and regulates the generation of cajal-retzius neurons in the developing cerebral cortex. Dev Neurosci 33, 479-493.

Cogliati, T., Good, D.J., Haigney, M., Delgado-Romero, P., Eckhaus, M.A., Koch, W.J., and Kirsch, I.R. (2002). Predisposition to arrhythmia and autonomic dysfunction in Nhlh1deficient mice. Mol Cell Biol 22, 4977-4983.

Cohen, P. (1982). The role of protein phosphorylation in neural and hormonal control of cellular activity. Nature 296, 613-620.

Collins, S.J. (2002). The role of retinoids and retinoic acid receptors in normal hematopoiesis. Leukemia 16, 1896-1905.

Connell, M., Cabernard, C., Ricketson, D., Doe, C.Q., and Prehoda, K.E. (2011). Asymmetric cortical extension shifts cleavage furrow position in Drosophila neuroblasts. Mol Biol Cell $22,4220-4226$.

Cox, D.B.T., Gootenberg, J.S., Abudayyeh, O.O., Franklin, B., Kellner, M.J., Joung, J., and Zhang, F. (2017). RNA editing with CRISPR-Cas13. Science 358, 1019-1027.

Cridge, A.G., Crowe-McAuliffe, C., Mathew, S.F., and Tate, W.P. (2018). Eukaryotic translational termination efficiency is influenced by the 3' nucleotides within the ribosomal mRNA channel. Nucleic Acids Res 46, 1927-1944.

D'Gama, A.M., and Walsh, C.A. (2018). Somatic mosaicism and neurodevelopmental disease. Nat Neurosci 21, 1504-1514.

Dehay, C., and Kennedy, H. (2007). Cell-cycle control and cortical development. Nat Rev Neurosci 8, 438-450. 
Dennis, D.J., Han, S., and Schuurmans, C. (2019). bHLH transcription factors in neural development, disease, and reprogramming. Brain Res 1705, 48-65.

Desir, J., Cassart, M., David, P., Van Bogaert, P., and Abramowicz, M. (2008). Primary microcephaly with ASPM mutation shows simplified cortical gyration with antero-posterior gradient pre- and post-natally. Am J Med Genet A 146A, 1439-1443.

Devakumar, D., Bamford, A., Ferreira, M.U., Broad, J., Rosch, R.E., Groce, N., Breuer, J., Cardoso, M.A., Copp, A.J., Alexandre, P., et al. (2018). Infectious causes of microcephaly: epidemiology, pathogenesis, diagnosis, and management. Lancet Infect Dis 18, e1-e13.

Dewing, P., Shi, T., Horvath, S., and Vilain, E. (2003). Sexually dimorphic gene expression in mouse brain precedes gonadal differentiation. Brain Res Mol Brain Res 118, 82-90.

Di Donato, N., Chiari, S., Mirzaa, G.M., Aldinger, K., Parrini, E., Olds, C., Barkovich, A.J., Guerrini, R., and Dobyns, W.B. (2017). Lissencephaly: Expanded imaging and clinical classification. Am J Med Genet A 173, 1473-1488.

Di Donato, N., Neuhann, T., Kahlert, A.K., Klink, B., Hackmann, K., Neuhann, I., Novotna, B., Schallner, J., Krause, C., Glass, I.A., et al. (2016). Mutations in EXOSC2 are associated with a novel syndrome characterised by retinitis pigmentosa, progressive hearing loss, premature ageing, short stature, mild intellectual disability and distinctive gestalt. J Med Genet 53, 419-425.

Di Giovambattista, A.P., Jacome Querejeta, I., Ventura Faci, P., Rodriguez Martinez, G., and Ramos Fuentes, F. (2017). [Familial EXOSC3-related pontocerebellar hypoplasia]. An Pediatr (Barc) 86, 284-286.

Ding, M., and Wang, X. (2017). Antagonism between Hedgehog and Wnt signaling pathways regulates tumorigenicity. Oncol Lett 14, 6327-6333.

Doma, M.K., and Parker, R. (2006). Endonucleolytic cleavage of eukaryotic mRNAs with stalls in translation elongation. Nature 440, 561-564.

Domingo-Prim, J., Endara-Coll, M., Bonath, F., Jimeno, S., Prados-Carvajal, R., Friedlander, M.R., Huertas, P., and Visa, N. (2019). EXOSC10 is required for RPA assembly and controlled DNA end resection at DNA double-strand breaks. Nat Commun 10, 2135.

Dominissini, D., Moshitch-Moshkovitz, S., Schwartz, S., Salmon-Divon, M., Ungar, L., Osenberg, S., Cesarkas, K., Jacob-Hirsch, J., Amariglio, N., Kupiec, M., et al. (2012). Topology of the human and mouse m6A RNA methylomes revealed by m6A-seq. Nature 485, 201-206.

Douglas, G., Cho, M.T., Telegrafi, A., Winter, S., Carmichael, J., Zackai, E.H., Deardorff, M.A., Harr, M., Williams, L., Psychogios, A., et al. (2018). De novo missense variants in MEIS2 recapitulate the microdeletion phenotype of cardiac and palate abnormalities, developmental delay, intellectual disability and dysmorphic features. Am J Med Genet A $176,1845-1851$.

Draganova, K., Zemke, M., Zurkirchen, L., Valenta, T., Cantu, C., Okoniewski, M., Schmid, M.T., Hoffmans, R., Gotz, M., Basler, K., et al. (2015). Wnt/beta-catenin signaling regulates sequential fate decisions of murine cortical precursor cells. Stem Cells 33, 170-182.

Economides, A.N., Frendewey, D., Yang, P., Dominguez, M.G., Dore, A.T., Lobov, I.B., Persaud, T., Rojas, J., McClain, J., Lengyel, P., et al. (2013). Conditionals by inversion provide a universal method for the generation of conditional alleles. Proc Natl Acad Sci U S A 110, E3179-3188.

Eggens, V.R., Barth, P.G., Niermeijer, J.M., Berg, J.N., Darin, N., Dixit, A., Fluss, J., Foulds, N., Fowler, D., Hortobagyi, T., et al. (2014). EXOSC3 mutations in pontocerebellar hypoplasia type 1: novel mutations and genotype-phenotype correlations. Orphanet J Rare Dis $9,23$.

Englund, C., Fink, A., Lau, C., Pham, D., Daza, R.A., Bulfone, A., Kowalczyk, T., and Hevner, R.F. (2005). Pax6, Tbr2, and Tbr1 are expressed sequentially by radial glia, intermediate progenitor cells, and postmitotic neurons in developing neocortex. J Neurosci 25, 247-251. 
Estivill-Torrus, G., Pearson, H., van Heyningen, V., Price, D.J., and Rashbass, P. (2002). Pax6 is required to regulate the cell cycle and the rate of progression from symmetrical to asymmetrical division in mammalian cortical progenitors. Development 129, 455-466.

Falk, S., Bonneau, F., Ebert, J., Kogel, A., and Conti, E. (2017). Mpp6 Incorporation in the Nuclear Exosome Contributes to RNA Channeling through the Mtr4 Helicase. Cell Rep 20, 2279-2286.

Fame, R.M., MacDonald, J.L., and Macklis, J.D. (2011). Development, specification, and diversity of callosal projection neurons. Trends Neurosci 34, 41-50.

Fan, X., Dong, J., Zhong, S., Wei, Y., Wu, Q., Yan, L., Yong, J., Sun, L., Wang, X., Zhao, Y., et al. (2018). Spatial transcriptomic survey of human embryonic cerebral cortex by singlecell RNA-seq analysis. Cell Res 28, 730-745.

Fasken, M.B., Losh, J.S., Leung, S.W., Brutus, S., Avin, B., Vaught, J.C., Potter-Birriel, J., Craig, T., Conn, G.L., Mills-Lujan, K., et al. (2017). Insight into the RNA Exosome Complex Through Modeling Pontocerebellar Hypoplasia Type 1b Disease Mutations in Yeast. Genetics 205, 221-237.

Fasken, M.B., Morton, D.J., Kuiper, E.G., Jones, S.K., Leung, S.W., and Corbett, A.H. (2020). The RNA Exosome and Human Disease. Methods Mol Biol 2062, 3-33.

Feng, Y., and Walsh, C.A. (2004). Mitotic spindle regulation by Nde1 controls cerebral cortical size. Neuron 44, 279-293.

Fietz, S.A., Kelava, I., Vogt, J., Wilsch-Brauninger, M., Stenzel, D., Fish, J.L., Corbeil, D., Riehn, A., Distler, W., Nitsch, R., et al. (2010). OSVZ progenitors of human and ferret neocortex are epithelial-like and expand by integrin signaling. Nat Neurosci 13, 690-699.

Fink, J.K. (2003). The hereditary spastic paraplegias: nine genes and counting. Arch Neurol 60, 1045-1049.

Fischer, G., Rossmann, M., and Hyvonen, M. (2015). Alternative modulation of protein-protein interactions by small molecules. Curr Opin Biotechnol 35, 78-85.

Flamand, M.N., and Meyer, K.D. (2019). The epitranscriptome and synaptic plasticity. Curr Opin Neurobiol 59, 41-48.

Florio, M., and Huttner, W.B. (2014). Neural progenitors, neurogenesis and the evolution of the neocortex. Development 141, 2182-2194.

Fossat, N., Tourle, K., Radziewic, T., Barratt, K., Liebhold, D., Studdert, J.B., Power, M., Jones, V., Loebel, D.A., and Tam, P.P. (2014). C to U RNA editing mediated by APOBEC1 requires RNA-binding protein RBM47. EMBO Rep 15, 903-910.

Frazao, C., McVey, C.E., Amblar, M., Barbas, A., Vonrhein, C., Arraiano, C.M., and Carrondo, M.A. (2006). Unravelling the dynamics of RNA degradation by ribonuclease II and its RNAbound complex. Nature 443, 110-114.

Freeman, M.V., Williams, D.W., Schimke, R.N., Temtamy, S.A., Vachier, E., and German, J. (1974). The Roberts syndrome. Birth Defects Orig Artic Ser 10, 87-95.

Fu, Y., Foden, J.A., Khayter, C., Maeder, M.L., Reyon, D., Joung, J.K., and Sander, J.D. (2013). High-frequency off-target mutagenesis induced by CRISPR-Cas nucleases in human cells. Nat Biotechnol 31, 822-826.

Fukuda, M., Umeno, H., Nose, K., Nishitarumizu, A., Noguchi, R., and Nakagawa, H. (2017). Construction of a guide-RNA for site-directed RNA mutagenesis utilising intracellular Ato-I RNA editing. Sci Rep 7, 41478.

German, J. (1979). Roberts' syndrome. I. Cytological evidence for a disturbance in chromatid pairing. Clin Genet 16, 441-447.

Geula, S., Moshitch-Moshkovitz, S., Dominissini, D., Mansour, A.A., Kol, N., Salmon-Divon, M., Hershkovitz, V., Peer, E., Mor, N., Manor, Y.S., et al. (2015). Stem cells. m6A mRNA methylation facilitates resolution of naive pluripotency toward differentiation. Science 347 , 1002-1006. 
Giliberti, A., Curro, A., Papa, F.T., Frullanti, E., Ariani, F., Coriolani, G., Grosso, S., Renieri, A., and Mari, F. (2019). MEIS2 gene is responsible for intellectual disability, cardiac defects and a distinct facial phenotype. Eur J Med Genet.

Giunta, M., Edvardson, S., Xu, Y., Schuelke, M., Gomez-Duran, A., Boczonadi, V., Elpeleg, O., Muller, J.S., and Horvath, R. (2016). Altered RNA metabolism due to a homozygous RBM7 mutation in a patient with spinal motor neuropathy. Hum Mol Genet 25, 2985-2996.

Glass, C.K., and Rosenfeld, M.G. (2000). The coregulator exchange in transcriptional functions of nuclear receptors. Genes Dev 14, 121-141.

Glickstein, S.B., Monaghan, J.A., Koeller, H.B., Jones, T.K., and Ross, M.E. (2009). Cyclin $\mathrm{D} 2$ is critical for intermediate progenitor cell proliferation in the embryonic cortex. $\mathrm{J}$ Neurosci 29, 9614-9624.

Glotzer, M. (2001). Animal cell cytokinesis. Annu Rev Cell Dev Biol 17, 351-386.

Goh, C.S., Milburn, D., and Gerstein, M. (2004). Conformational changes associated with protein-protein interactions. Curr Opin Struct Biol 14, 104-109.

Gordillo, M., Vega, H., Trainer, A.H., Hou, F., Sakai, N., Luque, R., Kayserili, H., Basaran, S., Skovby, F., Hennekam, R.C., et al. (2008). The molecular mechanism underlying Roberts syndrome involves loss of ESCO2 acetyltransferase activity. Hum Mol Genet 17, 21722180.

Gorski, J.A., Talley, T., Qiu, M., Puelles, L., Rubenstein, J.L., and Jones, K.R. (2002). Cortical excitatory neurons and glia, but not GABAergic neurons, are produced in the Emx1expressing lineage. J Neurosci 22, 6309-6314.

Gotz, M., and Huttner, W.B. (2005). The cell biology of neurogenesis. Nat Rev Mol Cell Biol 6, 777-788.

Green, D.R. (1998). Apoptotic pathways: the roads to ruin. Cell 94, 695-698.

Gribble, S.L., Kim, H.S., Bonner, J., Wang, X., and Dorsky, R.I. (2009). Tcf3 inhibits spinal cord neurogenesis by regulating sox4a expression. Development 136, 781-789.

Grill, S.W., Gonczy, P., Stelzer, E.H., and Hyman, A.A. (2001). Polarity controls forces governing asymmetric spindle positioning in the Caenorhabditis elegans embryo. Nature 409, 630-633.

Gudipati, R.K., Xu, Z., Lebreton, A., Seraphin, B., Steinmetz, L.M., Jacquier, A., and Libri, D. (2012). Extensive degradation of RNA precursors by the exosome in wild-type cells. Mol Cell 48, 409-421.

Gulacsi, A.A., and Anderson, S.A. (2008). Beta-catenin-mediated Wnt signaling regulates neurogenesis in the ventral telencephalon. Nat Neurosci 11, 1383-1391.

Guttinger, S., Laurell, E., and Kutay, U. (2009). Orchestrating nuclear envelope disassembly and reassembly during mitosis. Nat Rev Mol Cell Biol 10, 178-191.

Halbach, F., Reichelt, P., Rode, M., and Conti, E. (2013). The yeast ski complex: crystal structure and RNA channeling to the exosome complex. Cell 154, 814-826.

Halevy, A., Lerer, I., Cohen, R., Kornreich, L., Shuper, A., Gamliel, M., Zimerman, B.E., Korabi, I., Meiner, V., Straussberg, R., et al. (2014). Novel EXOSC3 mutation causes complicated hereditary spastic paraplegia. J Neurol 261, 2165-2169.

Han, J., Flemington, C., Houghton, A.B., Gu, Z., Zambetti, G.P., Lutz, R.J., Zhu, L., and Chittenden, T. (2001). Expression of bbc3, a pro-apoptotic BH3-only gene, is regulated by diverse cell death and survival signals. Proc Natl Acad Sci U S A 98, 11318-11323.

Hansen, D.V., Lui, J.H., Parker, P.R., and Kriegstein, A.R. (2010). Neurogenic radial glia in the outer subventricular zone of human neocortex. Nature 464, 554-561.

Haubensak, W., Attardo, A., Denk, W., and Huttner, W.B. (2004). Neurons arise in the basal neuroepithelium of the early mammalian telencephalon: a major site of neurogenesis. Proc Natl Acad Sci U S A 101, 3196-3201.

Haydar, T.F., Kuan, C.Y., Flavell, R.A., and Rakic, P. (1999). The role of cell death in regulating the size and shape of the mammalian forebrain. Cereb Cortex 9, 621-626. 
Hebert, J.M., and McConnell, S.K. (2000). Targeting of cre to the Foxg1 (BF-1) locus mediates loxP recombination in the telencephalon and other developing head structures. Dev Biol 222, 296-306.

Heins, N., Malatesta, P., Cecconi, F., Nakafuku, M., Tucker, K.L., Hack, M.A., Chapouton, P., Barde, Y.A., and Gotz, M. (2002). Glial cells generate neurons: the role of the transcription factor Pax6. Nat Neurosci 5, 308-315.

Helm, M., and Motorin, Y. (2017). Detecting RNA modifications in the epitranscriptome: predict and validate. Nat Rev Genet 18, 275-291.

Hevner, R.F. (2019). Intermediate progenitors and Tbr2 in cortical development. J Anat 235, 616-625.

Hevner, R.F., Shi, L., Justice, N., Hsueh, Y., Sheng, M., Smiga, S., Bulfone, A., Goffinet, A.M., Campagnoni, A.T., and Rubenstein, J.L. (2001). Tbr1 regulates differentiation of the preplate and layer 6. Neuron 29, 353-366.

Hochstrasser, M. (1995). Ubiquitin, proteasomes, and the regulation of intracellular protein degradation. Curr Opin Cell Biol 7, 215-223.

Holland, P.W., Booth, H.A., and Bruford, E.A. (2007). Classification and nomenclature of all human homeobox genes. BMC Biol 5, 47.

Hong, S.E., Shugart, Y.Y., Huang, D.T., Shahwan, S.A., Grant, P.E., Hourihane, J.O., Martin, N.D., and Walsh, C.A. (2000). Autosomal recessive lissencephaly with cerebellar hypoplasia is associated with human RELN mutations. Nat Genet 26, 93-96.

Hou, F., and Zou, H. (2005). Two human orthologues of Eco1/Ctf7 acetyltransferases are both required for proper sister-chromatid cohesion. Mol Biol Cell 16, 3908-3918.

Houseley, J., LaCava, J., and Tollervey, D. (2006). RNA-quality control by the exosome. Nat Rev Mol Cell Biol 7, 529-539.

Houseley, J., and Tollervey, D. (2009). The many pathways of RNA degradation. Cell 136, 763-776.

Hu, W.F., Chahrour, M.H., and Walsh, C.A. (2014). The diverse genetic landscape of neurodevelopmental disorders. Annu Rev Genomics Hum Genet 15, 195-213.

Huang da, W., Sherman, B.T., and Lempicki, R.A. (2009). Bioinformatics enrichment tools: paths toward the comprehensive functional analysis of large gene lists. Nucleic Acids Res $37,1-13$.

Huang, D.C., and Strasser, A. (2000). BH3-Only proteins-essential initiators of apoptotic cell death. Cell 103, 839-842.

Huber, L.A., and Teis, D. (2016). Lysosomal signaling in control of degradation pathways. Curr Opin Cell Biol 39, 8-14.

Hunter, T. (1995). Protein kinases and phosphatases: the yin and yang of protein phosphorylation and signaling. Cell 80, 225-236.

Huttner, W.B., and Brand, M. (1997). Asymmetric division and polarity of neuroepithelial cells. Curr Opin Neurobiol 7, 29-39.

Ikonomidou, C. (2009). Triggers of apoptosis in the immature brain. Brain Dev 31, 488-492.

Imamura, T., Yamamoto, S., Ohgane, J., Hattori, N., Tanaka, S., and Shiota, K. (2004). Noncoding RNA directed DNA demethylation of Sphk1 CpG island. Biochem Biophys Res Commun 322, 593-600.

Ivanov, I., Atkinson, D., Litvinenko, I., Angelova, L., Andonova, S., Mumdjiev, H., Pacheva, I., Panova, M., Yordanova, R., Belovejdov, V., et al. (2018). Pontocerebellar hypoplasia type 1 for the neuropediatrician: Genotype-phenotype correlations and diagnostic guidelines based on new cases and overview of the literature. Eur J Paediatr Neurol 22, 674-681.

Jabs, E.W., Tuck-Muller, C.M., Cusano, R., and Rattner, J.B. (1989). Centromere separation and aneuploidy in human mitotic mutants: Roberts syndrome. Prog Clin Biol Res 318, 111118.

Jain, A.K., and Barton, M.C. (2018). p53: emerging roles in stem cells, development and beyond. Development 145. 
Jamin, S.P., Petit, F.G., Kervarrec, C., Smagulova, F., Illner, D., Scherthan, H., and Primig, M. (2017). EXOSC10/Rrp6 is post-translationally regulated in male germ cells and controls the onset of spermatogenesis. Sci Rep 7, 15065.

Januszyk, K., and Lima, C.D. (2010). Structural components and architectures of RNA exosomes. Adv Exp Med Biol 702, 9-28.

Januszyk, K., and Lima, C.D. (2014). The eukaryotic RNA exosome. Curr Opin Struct Biol 24, 132-140.

Jeffers, J.R., Parganas, E., Lee, Y., Yang, C., Wang, J., Brennan, J., MacLean, K.H., Han, J., Chittenden, T., Ihle, J.N., et al. (2003). Puma is an essential mediator of p53-dependent and -independent apoptotic pathways. Cancer Cell 4, 321-328.

Jones, S. (2004). An overview of the basic helix-loop-helix proteins. Genome Biol 5, 226.

Juberg, R.C., and Hayward, J.R. (1969). A new familial syndrome of oral, cranial, and digital anomalies. J Pediatr 74, 755-762.

Juric-Sekhar, G., and Hevner, R.F. (2019). Malformations of Cerebral Cortex Development: Molecules and Mechanisms. Annu Rev Pathol 14, 293-318.

Kadaba, S., Krueger, A., Trice, T., Krecic, A.M., Hinnebusch, A.G., and Anderson, J. (2004). Nuclear surveillance and degradation of hypomodified initiator tRNAMet in S. cerevisiae. Genes Dev 18, 1227-1240.

Kadaba, S., Wang, X., and Anderson, J.T. (2006). Nuclear RNA surveillance in Saccharomyces cerevisiae: Trf4p-dependent polyadenylation of nascent hypomethylated tRNA and an aberrant form of 5S rRNA. RNA 12, 508-521.

Kang, H.J., Kawasawa, Y.I., Cheng, F., Zhu, Y., Xu, X., Li, M., Sousa, A.M., Pletikos, M., Meyer, K.A., Sedmak, G., et al. (2011). Spatio-temporal transcriptome of the human brain. Nature 478, 483-489.

Kantaputra, P.N., Dejkhamron, P., Intachai, W., Ngamphiw, C., Kawasaki, K., Ohazama, A., Krisanaprakornkit, S., Olsen, B., Tongsima, S., and Ketudat Cairns, J.R. (2020a). JubergHayward syndrome is a cohesinopathy, caused by mutation in ESCO2. Eur J Orthod.

Kantaputra, P.N., Dejkhamron, P., Tongsima, S., Ngamphiw, C., Intachai, W., Ngiwsara, L., Sawangareetrakul, P., Svasti, J., Olsen, B., Cairns, J.R.K., et al. (2020b). Juberg-Hayward syndrome and Roberts syndrome are allelic, caused by mutations in ESCO2. Arch Oral Biol 119, 104918.

Kantaputra, P.N., and Mongkolchaisup, S. (1999). Juberg-Hayward syndrome: a new case report and clinical delineation of the syndrome. Clin Dysmorphol 8, 123-127.

Kapoor, T.M., and Compton, D.A. (2002). Searching for the middle ground: mechanisms of chromosome alignment during mitosis. J Cell Biol 157, 551-556.

Kasowitz, S.D., Ma, J., Anderson, S.J., Leu, N.A., Xu, Y., Gregory, B.D., Schultz, R.M., and Wang, P.J. (2018). Nuclear m6A reader YTHDC1 regulates alternative polyadenylation and splicing during mouse oocyte development. PLoS Genet 14, e1007412.

Katrekar, D., Chen, G., Meluzzi, D., Ganesh, A., Worlikar, A., Shih, Y.R., Varghese, S., and Mali, P. (2019). In vivo RNA editing of point mutations via RNA-guided adenosine deaminases. Nat Methods 16, 239-242.

Kawaguchi, A., Ikawa, T., Kasukawa, T., Ueda, H.R., Kurimoto, K., Saitou, M., and Matsuzaki, F. (2008). Single-cell gene profiling defines differential progenitor subclasses in mammalian neurogenesis. Development 135, 3113-3124.

Kawase, T., Ichikawa, H., Ohta, T., Nozaki, N., Tashiro, F., Ohki, R., and Taya, Y. (2008). p53 target gene AEN is a nuclear exonuclease required for p53-dependent apoptosis. Oncogene 27, 3797-3810.

Kilchert, C., Wittmann, S., Passoni, M., Shah, S., Granneman, S., and Vasiljeva, L. (2015). Regulation of mRNA Levels by Decay-Promoting Introns that Recruit the Exosome Specificity Factor Mmi1. Cell Rep 13, 2504-2515.

Kilchert, C., Wittmann, S., and Vasiljeva, L. (2016). The regulation and functions of the nuclear RNA exosome complex. Nat Rev Mol Cell Biol 17, 227-239. 
Kingston, H.M., Hughes, I.A., and Harper, P.S. (1982). Orocraniodigital (Juberg-Hayward) syndrome with growth hormone deficiency. Arch Dis Child 57, 790-792.

Kitamura, K., Yanazawa, M., Sugiyama, N., Miura, H., Iizuka-Kogo, A., Kusaka, M., Omichi, K., Suzuki, R., Kato-Fukui, Y., Kamiirisa, K., et al. (2002). Mutation of ARX causes abnormal development of forebrain and testes in mice and X-linked lissencephaly with abnormal genitalia in humans. Nat Genet 32, 359-369.

Knight, J.R., Bastide, A., Peretti, D., Roobol, A., Roobol, J., Mallucci, G.R., Smales, C.M., and Willis, A.E. (2016). Cooling-induced SUMOylation of EXOSC10 down-regulates ribosome biogenesis. RNA 22, 623-635.

Knoblich, J.A. (2001). Asymmetric cell division during animal development. Nat Rev Mol Cell Biol 2, 11-20.

Koepp, D.M. (2014). Cell cycle regulation by protein degradation. Methods Mol Biol 1170, 6173.

Komada, M. (2012). Sonic hedgehog signaling coordinates the proliferation and differentiation of neural stem/progenitor cells by regulating cell cycle kinetics during development of the neocortex. Congenit Anom (Kyoto) 52, 72-77.

Komada, M., Saitsu, H., Kinboshi, M., Miura, T., Shiota, K., and Ishibashi, M. (2008). Hedgehog signaling is involved in development of the neocortex. Development 135, $2717-$ 2727.

Komarov, P.G., Komarova, E.A., Kondratov, R.V., Christov-Tselkov, K., Coon, J.S., Chernov, M.V., and Gudkov, A.V. (1999). A chemical inhibitor of p53 that protects mice from the side effects of cancer therapy. Science 285, 1733-1737.

Komuro, H., and Rakic, P. (1998). Distinct modes of neuronal migration in different domains of developing cerebellar cortex. J Neurosci 18, 1478-1490.

Kosodo, Y., Roper, K., Haubensak, W., Marzesco, A.M., Corbeil, D., and Huttner, W.B. (2004). Asymmetric distribution of the apical plasma membrane during neurogenic divisions of mammalian neuroepithelial cells. EMBO J 23, 2314-2324.

Kostic, M., Paridaen, J., Long, K.R., Kalebic, N., Langen, B., Grubling, N., Wimberger, P., Kawasaki, H., Namba, T., and Huttner, W.B. (2019). YAP Activity Is Necessary and Sufficient for Basal Progenitor Abundance and Proliferation in the Developing Neocortex. Cell Rep 27, 1103-1118 e1106.

Kousar, R., Nawaz, H., Khurshid, M., Ali, G., Khan, S.U., Mir, H., Ayub, M., Wali, A., Ali, N., Jelani, M., et al. (2010). Mutation analysis of the ASPM gene in 18 Pakistani families with autosomal recessive primary microcephaly. J Child Neurol 25, 715-720.

Kowalczyk, T., Pontious, A., Englund, C., Daza, R.A., Bedogni, F., Hodge, R., Attardo, A., Bell, C., Huttner, W.B., and Hevner, R.F. (2009). Intermediate neuronal progenitors (basal progenitors) produce pyramidal-projection neurons for all layers of cerebral cortex. Cereb Cortex 19, 2439-2450.

Kriegstein, A., Noctor, S., and Martinez-Cerdeno, V. (2006). Patterns of neural stem and progenitor cell division may underlie evolutionary cortical expansion. Nat Rev Neurosci 7, 883-890.

Kriegstein, A.R., and Noctor, S.C. (2004). Patterns of neuronal migration in the embryonic cortex. Trends Neurosci 27, 392-399.

Kuida, K., Haydar, T.F., Kuan, C.Y., Gu, Y., Taya, C., Karasuyama, H., Su, M.S., Rakic, P., and Flavell, R.A. (1998). Reduced apoptosis and cytochrome c-mediated caspase activation in mice lacking caspase 9. Cell 94, 325-337.

Kuida, K., Zheng, T.S., Na, S., Kuan, C., Yang, D., Karasuyama, H., Rakic, P., and Flavell, R.A. (1996). Decreased apoptosis in the brain and premature lethality in CPP32-deficient mice. Nature 384, 368-372.

Kumar, A., Girimaji, S.C., Duvvari, M.R., and Blanton, S.H. (2009). Mutations in STIL, encoding a pericentriolar and centrosomal protein, cause primary microcephaly. Am J Hum Genet 84, 286-290. 
Kumar, R.A., Pilz, D.T., Babatz, T.D., Cushion, T.D., Harvey, K., Topf, M., Yates, L., Robb, S., Uyanik, G., Mancini, G.M., et al. (2010). TUBA1A mutations cause wide spectrum lissencephaly (smooth brain) and suggest that multiple neuronal migration pathways converge on alpha tubulins. Hum Mol Genet 19, 2817-2827.

LaCava, J., Houseley, J., Saveanu, C., Petfalski, E., Thompson, E., Jacquier, A., and Tollervey, D. (2005). RNA degradation by the exosome is promoted by a nuclear polyadenylation complex. Cell 121, 713-724.

Lange, C., Huttner, W.B., and Calegari, F. (2009). Cdk4/cyclinD1 overexpression in neural stem cells shortens G1, delays neurogenesis, and promotes the generation and expansion of basal progenitors. Cell Stem Cell 5, 320-331.

Le Duc, D., Horn, S., Jamra, R.A., Schaper, J., Wieczorek, D., and Redler, S. (2020). Novel EXOSC3 pathogenic variant results in a mild course of neurologic disease with cerebellum involvement. Eur J Med Genet 63, 103649.

Le Hellard, S., Muhleisen, T.W., Djurovic, S., Ferno, J., Ouriaghi, Z., Mattheisen, M., Vasilescu, C., Raeder, M.B., Hansen, T., Strohmaier, J., et al. (2010). Polymorphisms in SREBF1 and SREBF2, two antipsychotic-activated transcription factors controlling cellular lipogenesis, are associated with schizophrenia in German and Scandinavian samples. Mol Psychiatry 15, 463-472.

Lebreton, A., Tomecki, R., Dziembowski, A., and Seraphin, B. (2008). Endonucleolytic RNA cleavage by a eukaryotic exosome. Nature 456, 993-996.

Lee, J.H., Koh, Y.A., Cho, C.K., Lee, S.J., Lee, Y.S., and Bae, S. (2005). Identification of a novel ionizing radiation-induced nuclease, $\mathrm{AEN}$, and its functional characterization in apoptosis. Biochem Biophys Res Commun 337, 39-47.

Lejeune, F., Li, X., and Maquat, L.E. (2003). Nonsense-mediated mRNA decay in mammalian cells involves decapping, deadenylating, and exonucleolytic activities. Mol Cell 12, 675687.

Lessard, J., Wu, J.I., Ranish, J.A., Wan, M., Winslow, M.M., Staahl, B.T., Wu, H., Aebersold, R., Graef, I.A., and Crabtree, G.R. (2007). An essential switch in subunit composition of a chromatin remodeling complex during neural development. Neuron 55, 201-215.

Letinic, K., Zoncu, R., and Rakic, P. (2002). Origin of GABAergic neurons in the human neocortex. Nature 417, 645-649.

Li, C.M., Yan, R.T., and Wang, S.Z. (1999). Misexpression of a bHLH gene, cNSCL1, results in abnormal brain development. Dev Dyn 215, 238-247.

Li, J., Chen, Z., Chen, F., Xie, G., Ling, Y., Peng, Y., Lin, Y., Luo, N., Chiang, C.M., and Wang, H. (2020a). Targeted mRNA demethylation using an engineered dCas13b-ALKBH5 fusion protein. Nucleic Acids Res 48, 5684-5694.

Li, M., Santpere, G., Imamura Kawasawa, Y., Evgrafov, O.V., Gulden, F.O., Pochareddy, S., Sunkin, S.M., Li, Z., Shin, Y., Zhu, Y., et al. (2018a). Integrative functional genomic analysis of human brain development and neuropsychiatric risks. Science 362.

Li, M., Zhao, X., Wang, W., Shi, H., Pan, Q., Lu, Z., Perez, S.P., Suganthan, R., He, C., Bjoras, M., et al. (2018b). Ythdf2-mediated m(6)A mRNA clearance modulates neural development in mice. Genome Biol 19, 69.

Li, X.J., Zhang, X., Johnson, M.A., Wang, Z.B., Lavaute, T., and Zhang, S.C. (2009). Coordination of sonic hedgehog and Wnt signaling determines ventral and dorsal telencephalic neuron types from human embryonic stem cells. Development 136, 40554063.

Li, Z., Tyler, W.A., Zeldich, E., Santpere Baro, G., Okamoto, M., Gao, T., Li, M., Sestan, N., and Haydar, T.F. (2020b). Transcriptional priming as a conserved mechanism of lineage diversification in the developing mouse and human neocortex. Sci Adv 6.

Lim, J., Giri, P.K., Kazadi, D., Laffleur, B., Zhang, W., Grinstein, V., Pefanis, E., Brown, L.M., Ladewig, E., Martin, O., et al. (2017). Nuclear Proximity of Mtr4 to RNA Exosome Restricts DNA Mutational Asymmetry. Cell 169, 523-537 e515. 
Liu, J., Yue, Y., Han, D., Wang, X., Fu, Y., Zhang, L., Jia, G., Yu, M., Lu, Z., Deng, X., et al. (2014). A METTL3-METTL14 complex mediates mammalian nuclear RNA N6-adenosine methylation. Nat Chem Biol 10, 93-95.

Liu, Q., Greimann, J.C., and Lima, C.D. (2006). Reconstitution, activities, and structure of the eukaryotic RNA exosome. Cell 127, 1223-1237.

Liu, S.J., Nowakowski, T.J., Pollen, A.A., Lui, J.H., Horlbeck, M.A., Attenello, F.J., He, D., Weissman, J.S., Kriegstein, A.R., Diaz, A.A., et al. (2016). Single-cell analysis of long noncoding RNAs in the developing human neocortex. Genome Biol 17, 67.

Liu, Z., Macias, M.J., Bottomley, M.J., Stier, G., Linge, J.P., Nilges, M., Bork, P., and Sattler, M. (1999). The three-dimensional structure of the HRDC domain and implications for the Werner and Bloom syndrome proteins. Structure 7, 1557-1566.

Lloret-Llinares, M., Mapendano, C.K., Martlev, L.H., Lykke-Andersen, S., and Jensen, T.H. (2016). Relationships between PROMPT and gene expression. RNA Biol 13, 6-14.

Logan, C.Y., and Nusse, R. (2004). The Wnt signaling pathway in development and disease. Annu Rev Cell Dev Biol 20, 781-810.

Lomonosova, E., and Chinnadurai, G. (2008). BH3-only proteins in apoptosis and beyond: an overview. Oncogene 27 Suppl 1, S2-19.

Loo, L., Simon, J.M., Xing, L., McCoy, E.S., Niehaus, J.K., Guo, J., Anton, E.S., and Zylka, M.J. (2019). Single-cell transcriptomic analysis of mouse neocortical development. Nat Commun 10, 134.

Lorentzen, E., Basquin, J., Tomecki, R., Dziembowski, A., and Conti, E. (2008). Structure of the active subunit of the yeast exosome core, Rrp44: diverse modes of substrate recruitment in the RNase II nuclease family. Mol Cell 29, 717-728.

Lorentzen, E., Walter, P., Fribourg, S., Evguenieva-Hackenberg, E., Klug, G., and Conti, E. (2005). The archaeal exosome core is a hexameric ring structure with three catalytic subunits. Nat Struct Mol Biol 12, 575-581.

Lou, F., Li, M., Liu, N., Li, X., Ren, Y., and Luo, X. (2019). The polymorphism of SREBF1 gene rs $11868035 \mathrm{G} / \mathrm{A}$ is associated with susceptibility to Parkinson's disease in a Chinese population. Int J Neurosci 129, 660-665.

Louw, J.J., Corveleyn, A., Jia, Y., Hens, G., Gewillig, M., and Devriendt, K. (2015). MEIS2 involvement in cardiac development, cleft palate, and intellectual disability. Am J Med Genet A 167A, 1142-1146.

Love, M.I., Huber, W., and Anders, S. (2014). Moderated estimation of fold change and dispersion for RNA-seq data with DESeq2. Genome Biol 15, 550.

Lu, T., Wu, B., Yu, Y., Zhu, W., Zhang, S., Zhang, Y., Guo, J., and Deng, N. (2018). Blockade of ONECUT2 expression in ovarian cancer inhibited tumor cell proliferation, migration, invasion and angiogenesis. Cancer Sci 109, 2221-2234.

Lubas, M., Christensen, M.S., Kristiansen, M.S., Domanski, M., Falkenby, L.G., LykkeAndersen, S., Andersen, J.S., Dziembowski, A., and Jensen, T.H. (2011). Interaction profiling identifies the human nuclear exosome targeting complex. Mol Cell 43, 624-637.

Lui, J.H., Hansen, D.V., and Kriegstein, A.R. (2011). Development and evolution of the human neocortex. Cell 146, 18-36.

Lukaszewicz, A., Savatier, P., Cortay, V., Giroud, P., Huissoud, C., Berland, M., Kennedy, H., and Dehay, C. (2005). G1 phase regulation, area-specific cell cycle control, and cytoarchitectonics in the primate cortex. Neuron 47, 353-364.

MacDonald, B.T., Tamai, K., and He, X. (2009). Wnt/beta-catenin signaling: components, mechanisms, and diseases. Dev Cell 17, 9-26.

Machold, R., Hayashi, S., Rutlin, M., Muzumdar, M.D., Nery, S., Corbin, J.G., Gritli-Linde, A., Dellovade, T., Porter, J.A., Rubin, L.L., et al. (2003). Sonic hedgehog is required for progenitor cell maintenance in telencephalic stem cell niches. Neuron 39, 937-950. 
Machon, O., Backman, M., Machonova, O., Kozmik, Z., Vacik, T., Andersen, L., and Krauss, S. (2007). A dynamic gradient of Wnt signaling controls initiation of neurogenesis in the mammalian cortex and cellular specification in the hippocampus. Dev Biol 311, 223-237.

Macosko, E.Z., Basu, A., Satija, R., Nemesh, J., Shekhar, K., Goldman, M., Tirosh, I., Bialas, A.R., Kamitaki, N., Martersteck, E.M., et al. (2015). Highly Parallel Genome-wide Expression Profiling of Individual Cells Using Nanoliter Droplets. Cell 161, 1202-1214.

Magdaleno, S., Keshvara, L., and Curran, T. (2002). Rescue of ataxia and preplate splitting by ectopic expression of Reelin in reeler mice. Neuron 33, 573-586.

Makino, D.L., Baumgartner, M., and Conti, E. (2013). Crystal structure of an RNA-bound 11subunit eukaryotic exosome complex. Nature 495, 70-75.

Malatesta, P., Hartfuss, E., and Gotz, M. (2000). Isolation of radial glial cells by fluorescentactivated cell sorting reveals a neuronal lineage. Development 127, 5253-5263.

Malecki, M., Viegas, S.C., Carneiro, T., Golik, P., Dressaire, C., Ferreira, M.G., and Arraiano, C.M. (2013). The exoribonuclease Dis3L2 defines a novel eukaryotic RNA degradation pathway. EMBO J 32, 1842-1854.

Mani, S., Radhakrishnan, S., Cheramangalam, R.N., Harkar, S., Rajendran, S., and Ramanan, N. (2020). Shh-Mediated Increase in beta-Catenin Levels Maintains Cerebellar Granule Neuron Progenitors in Proliferation. Cerebellum 19, 645-664.

Marin-Padilla, M. (1978). Dual origin of the mammalian neocortex and evolution of the cortical plate. Anat Embryol (Berl) 152, 109-126.

Marin-Vicente, C., Domingo-Prim, J., Eberle, A.B., and Visa, N. (2015). RRP6/EXOSC10 is required for the repair of DNA double-strand breaks by homologous recombination. J Cell Sci $128,1097-1107$.

Martinez-Cerdeno, V., Noctor, S.C., and Kriegstein, A.R. (2006). The role of intermediate progenitor cells in the evolutionary expansion of the cerebral cortex. Cereb Cortex $16 \mathrm{Suppl}$ $1, \mathrm{i} 152-161$.

Martinez-Nunez, R.T., Wallace, A., Coyne, D., Jansson, L., Rush, M., Ennajdaoui, H., Katzman, S., Bailey, J., Deinhardt, K., Sanchez-Elsner, T., et al. (2017). Modulation of nonsense mediated decay by rapamycin. Nucleic Acids Res 45, 3448-3459.

McIntosh, J.R., Grishchuk, E.L., and West, R.R. (2002). Chromosome-microtubule interactions during mitosis. Annu Rev Cell Dev Biol 18, 193-219.

McIver, S.C., Kang, Y.A., DeVilbiss, A.W., O'Driscoll, C.A., Ouellette, J.N., Pope, N.J., Camprecios, G., Chang, C.J., Yang, D., Bouhassira, E.E., et al. (2014). The exosome complex establishes a barricade to erythroid maturation. Blood 124, 2285-2297.

McIver, S.C., Katsumura, K.R., Davids, E., Liu, P., Kang, Y.A., Yang, D., and Bresnick, E.H. (2016). Exosome complex orchestrates developmental signaling to balance proliferation and differentiation during erythropoiesis. Elife 5.

Melcher, T., Maas, S., Herb, A., Sprengel, R., Higuchi, M., and Seeburg, P.H. (1996a). RED2, a brain-specific member of the RNA-specific adenosine deaminase family. J Biol Chem 271, 31795-31798.

Melcher, T., Maas, S., Herb, A., Sprengel, R., Seeburg, P.H., and Higuchi, M. (1996b). A mammalian RNA editing enzyme. Nature 379, 460-464.

Mendrysa, S.M., Ghassemifar, S., and Malek, R. (2011). p53 in the CNS: Perspectives on Development, Stem Cells, and Cancer. Genes Cancer 2, 431-442.

Mengen, E., Kotan, L.D., Ucakturk, S.A., Topaloglu, A.K., and Yuksel, B. (2018). A Novel Frameshift Mutation in ESCO2 Gene in Roberts Syndrome. J Coll Physicians Surg Pak 28, 403-405.

Merkle, T., Merz, S., Reautschnig, P., Blaha, A., Li, Q., Vogel, P., Wettengel, J., Li, J.B., and Stafforst, T. (2019). Precise RNA editing by recruiting endogenous ADARs with antisense oligonucleotides. Nat Biotechnol 37, 133-138. 
Methot, L., Hermann, R., Tang, Y., Lo, R., Al-Jehani, H., Jhas, S., Svoboda, D., Slack, R.S., Barker, P.A., and Stifani, S. (2013). Interaction and antagonistic roles of NF-kappaB and Hes6 in the regulation of cortical neurogenesis. Mol Cell Biol 33, 2797-2808.

Meyer, K.D., Patil, D.P., Zhou, J., Zinoviev, A., Skabkin, M.A., Elemento, O., Pestova, T.V., Qian, S.B., and Jaffrey, S.R. (2015). 5' UTR m(6)A Promotes Cap-Independent Translation. Cell 163, 999-1010.

Meyer, K.D., Saletore, Y., Zumbo, P., Elemento, O., Mason, C.E., and Jaffrey, S.R. (2012). Comprehensive analysis of mRNA methylation reveals enrichment in 3' UTRs and near stop codons. Cell 149, 1635-1646.

Mian, I.S. (1997). Comparative sequence analysis of ribonucleases HII, III, II PH and D. Nucleic Acids Res 25, 3187-3195.

Mierzwa, B., and Gerlich, D.W. (2014). Cytokinetic abscission: molecular mechanisms and temporal control. Dev Cell 31, 525-538.

Mihalas, A.B., Elsen, G.E., Bedogni, F., Daza, R.A.M., Ramos-Laguna, K.A., Arnold, S.J., and Hevner, R.F. (2016). Intermediate Progenitor Cohorts Differentially Generate Cortical Layers and Require Tbr2 for Timely Acquisition of Neuronal Subtype Identity. Cell Rep 16, 92-105.

Mihalas, A.B., and Hevner, R.F. (2018). Clonal analysis reveals laminar fate multipotency and daughter cell apoptosis of mouse cortical intermediate progenitors. Development 145 .

Miller, J.A., Ding, S.L., Sunkin, S.M., Smith, K.A., Ng, L., Szafer, A., Ebbert, A., Riley, Z.L., Royall, J.J., Aiona, K., et al. (2014). Transcriptional landscape of the prenatal human brain. Nature 508, 199-206.

Mistry, D.S., Chen, Y., and Sen, G.L. (2012). Progenitor function in self-renewing human epidermis is maintained by the exosome. Cell Stem Cell 11, 127-135.

Mitchell, P., Petfalski, E., Shevchenko, A., Mann, M., and Tollervey, D. (1997). The exosome: a conserved eukaryotic RNA processing complex containing multiple $3^{\prime}-->5^{\prime}$ exoribonucleases. Cell 91, 457-466.

Mitchell, P., Petfalski, E., and Tollervey, D. (1996). The 3' end of yeast 5.8S rRNA is generated by an exonuclease processing mechanism. Genes Dev 10, 502-513.

Mitchell, P., and Tollervey, D. (2003). An NMD pathway in yeast involving accelerated deadenylation and exosome-mediated 3'-->5' degradation. Mol Cell 11, 1405-1413.

Mitchison, T.J., and Salmon, E.D. (2001). Mitosis: a history of division. Nat Cell Biol 3, E1721.

Miyata, T., Kawaguchi, A., Saito, K., Kawano, M., Muto, T., and Ogawa, M. (2004). Asymmetric production of surface-dividing and non-surface-dividing cortical progenitor cells. Development 131, 3133-3145.

Mo, J., Chen, Z., Qin, S., Li, S., Liu, C., Zhang, L., Ran, R., Kong, Y., Wang, F., Liu, S., et al. (2020). TRADES: Targeted RNA Demethylation by SunTag System. Adv Sci (Weinh) 7, 2001402.

Molliver, M.E., Kostovic, I., and van der Loos, H. (1973). The development of synapses in cerebral cortex of the human fetus. Brain Res 50, 403-407.

Molyneaux, B.J., Arlotta, P., Menezes, J.R., and Macklis, J.D. (2007). Neuronal subtype specification in the cerebral cortex. Nat Rev Neurosci 8, 427-437.

Molyneaux, B.J., Goff, L.A., Brettler, A.C., Chen, H.H., Hrvatin, S., Rinn, J.L., and Arlotta, P. (2015). DeCoN: genome-wide analysis of in vivo transcriptional dynamics during pyramidal neuron fate selection in neocortex. Neuron 85, 275-288.

Montiel-Gonzalez, M.F., Vallecillo-Viejo, I., Yudowski, G.A., and Rosenthal, J.J. (2013). Correction of mutations within the cystic fibrosis transmembrane conductance regulator by site-directed RNA editing. Proc Natl Acad Sci U S A 110, 18285-18290.

Morton, D.J., Kuiper, E.G., Jones, S.K., Leung, S.W., Corbett, A.H., and Fasken, M.B. (2018). The RNA exosome and RNA exosome-linked disease. Rna 24, 127-142. 
Mukherjee, D., Gao, M., O'Connor, J.P., Raijmakers, R., Pruijn, G., Lutz, C.S., and Wilusz, J. (2002). The mammalian exosome mediates the efficient degradation of mRNAs that contain AU-rich elements. EMBO J 21, 165-174.

Muller, J.S., Burns, D.T., Griffin, H., Wells, G.R., Zendah, R.A., Munro, B., Schneider, C., and Horvath, R. (2020). RNA exosome mutations in pontocerebellar hypoplasia alter ribosome biogenesis and p53 levels. Life Sci Alliance 3.

Mullor, J.L., Dahmane, N., Sun, T., and Ruiz i Altaba, A. (2001). Wnt signals are targets and mediators of Gli function. Curr Biol 11, 769-773.

Munji, R.N., Choe, Y., Li, G., Siegenthaler, J.A., and Pleasure, S.J. (2011). Wnt signaling regulates neuronal differentiation of cortical intermediate progenitors. J Neurosci 31, 16761687.

Murao, N., Matsubara, S., Matsuda, T., Noguchi, H., Mutoh, T., Mutoh, M., Koseki, H., Namihira, M., and Nakashima, K. (2019). Np95/Uhrf1 regulates tumor suppressor gene expression of neural stem/precursor cells, contributing to neurogenesis in the adult mouse brain. Neurosci Res 143, 31-43.

Musa, J., Aynaud, M.M., Mirabeau, O., Delattre, O., and Grunewald, T.G. (2017). MYBL2 (B$\mathrm{Myb}$ ): a central regulator of cell proliferation, cell survival and differentiation involved in tumorigenesis. Cell Death Dis 8, e2895.

Muzio, L., Di Benedetto, B., Stoykova, A., Boncinelli, E., Gruss, P., and Mallamaci, A. (2002). Conversion of cerebral cortex into basal ganglia in Emx2(-/-) Pax6(Sey/Sey) double-mutant mice. Nat Neurosci 5, 737-745.

Nachtergaele, S., and He, C. (2018). Chemical Modifications in the Life of an mRNA Transcript. Annu Rev Genet 52, 349-372.

Nagarajan, V.K., Jones, C.I., Newbury, S.F., and Green, P.J. (2013). XRN 5'-->3' exoribonucleases: structure, mechanisms and functions. Biochim Biophys Acta 1829, 590603.

Nakamura, I., Fernandez-Barrena, M.G., Ortiz-Ruiz, M.C., Almada, L.L., Hu, C., Elsawa, S.F., Mills, L.D., Romecin, P.A., Gulaid, K.H., Moser, C.D., et al. (2013). Activation of the transcription factor GLI1 by WNT signaling underlies the role of SULFATASE 2 as a regulator of tissue regeneration. J Biol Chem 288, 21389-21398.

Nam, S.M., Kim, Y.N., Kim, J.W., Kyeong, D.S., Lee, S.H., Son, Y., Shin, J.H., Kim, J., Yi, S.S., Yoon, Y.S., et al. (2016). Hairy and Enhancer of Split 6 (Hes6) Deficiency in Mouse Impairs Neuroblast Differentiation in Dentate Gyrus Without Affecting Cell Proliferation and Integration into Mature Neurons. Cell Mol Neurobiol 36, 57-67.

Namavar, Y., Barth, P.G., Poll-The, B.T., and Baas, F. (2011). Classification, diagnosis and potential mechanisms in pontocerebellar hypoplasia. Orphanet J Rare Dis 6, 50.

Narayanan, R., Pham, L., Kerimoglu, C., Watanabe, T., Castro Hernandez, R., Sokpor, G., Ulmke, P.A., Kiszka, K.A., Tonchev, A.B., Rosenbusch, J., et al. (2018). Chromatin Remodeling BAF155 Subunit Regulates the Genesis of Basal Progenitors in Developing Cortex. iScience 4, 109-126.

Narayanan, R., Pirouz, M., Kerimoglu, C., Pham, L., Wagener, R.J., Kiszka, K.A., Rosenbusch, J., Seong, R.H., Kessel, M., Fischer, A., et al. (2015). Loss of BAF (mSWI/SNF) Complexes Causes Global Transcriptional and Chromatin State Changes in Forebrain Development. Cell Rep 13, 1842-1854.

Narayanan, R., and Tuoc, T.C. (2014). Roles of chromatin remodeling BAF complex in neural differentiation and reprogramming. Cell Tissue Res 356, 575-584.

Nasmyth, K., and Haering, C.H. (2009). Cohesin: its roles and mechanisms. Annu Rev Genet 43, 525-558.

Nevin, N.C., Henry, P., and Thomas, P.T. (1981). A case of the orocraniodigital (JubergHayward) syndrome. J Med Genet 18, 478-480.

Nguyen, H., Kerimoglu, C., Pirouz, M., Pham, L., Kiszka, K.A., Sokpor, G., Sakib, M.S., Rosenbusch, J., Teichmann, U., Seong, R.H., et al. (2018). Epigenetic Regulation by BAF 
Complexes Limits Neural Stem Cell Proliferation by Suppressing Wnt Signaling in Late Embryonic Development. Stem Cell Reports 10, 1734-1750.

Nishikura, K. (2016). A-to-I editing of coding and non-coding RNAs by ADARs. Nat Rev Mol Cell Biol 17, 83-96.

Nishioka, K., Hayashi, S., Farrer, M.J., Singleton, A.B., Yoshino, H., Imai, H., Kitami, T., Sato, K., Kuroda, R., Tomiyama, H., et al. (2006). Clinical heterogeneity of alpha-synuclein gene duplication in Parkinson's disease. Ann Neurol 59, 298-309.

Nishiyama, T., Ladurner, R., Schmitz, J., Kreidl, E., Schleiffer, A., Bhaskara, V., Bando, M., Shirahige, K., Hyman, A.A., Mechtler, K., et al. (2010). Sororin mediates sister chromatid cohesion by antagonizing Wapl. Cell 143, 737-749.

Noctor, S.C., Flint, A.C., Weissman, T.A., Dammerman, R.S., and Kriegstein, A.R. (2001). Neurons derived from radial glial cells establish radial units in neocortex. Nature 409, 714720.

Noctor, S.C., Flint, A.C., Weissman, T.A., Wong, W.S., Clinton, B.K., and Kriegstein, A.R. (2002). Dividing precursor cells of the embryonic cortical ventricular zone have morphological and molecular characteristics of radial glia. J Neurosci 22, 3161-3173.

Noctor, S.C., Martinez-Cerdeno, V., Ivic, L., and Kriegstein, A.R. (2004). Cortical neurons arise in symmetric and asymmetric division zones and migrate through specific phases. Nat Neurosci 7, 136-144.

Noubissi, F.K., Goswami, S., Sanek, N.A., Kawakami, K., Minamoto, T., Moser, A., Grinblat, Y., and Spiegelman, V.S. (2009). Wnt signaling stimulates transcriptional outcome of the Hedgehog pathway by stabilizing GLI1 mRNA. Cancer Res 69, 8572-8578.

Nowakowski, T.J., Bhaduri, A., Pollen, A.A., Alvarado, B., Mostajo-Radji, M.A., Di Lullo, E., Haeussler, M., Sandoval-Espinosa, C., Liu, S.J., Velmeshev, D., et al. (2017). Spatiotemporal gene expression trajectories reveal developmental hierarchies of the human cortex. Science 358, 1318-1323.

Oliferenko, S., Chew, T.G., and Balasubramanian, M.K. (2009). Positioning cytokinesis. Genes Dev 23, 660-674.

Oliveira Melo, A.S., Malinger, G., Ximenes, R., Szejnfeld, P.O., Alves Sampaio, S., and Bispo de Filippis, A.M. (2016). Zika virus intrauterine infection causes fetal brain abnormality and microcephaly: tip of the iceberg? Ultrasound Obstet Gynecol 47, 6-7.

Oliveros, J. 2007-2015. Venny. An interactive tool for comparing lists with Venn's diagrams.

Onn, I., Heidinger-Pauli, J.M., Guacci, V., Unal, E., and Koshland, D.E. (2008). Sister chromatid cohesion: a simple concept with a complex reality. Annu Rev Cell Dev Biol 24, 105-129.

Ouyang, T., Meng, W., Li, M., Hong, T., and Zhang, N. (2020). Recent Advances of the Hippo/YAP Signaling Pathway in Brain Development and Glioma. Cell Mol Neurobiol 40, 495-510.

Papari, E., Bastami, M., Farhadi, A., Abedini, S.S., Hosseini, M., Bahman, I., Mohseni, M., Garshasbi, M., Moheb, L.A., Behjati, F., et al. (2013). Investigation of primary microcephaly in Bushehr province of Iran: novel STIL and ASPM mutations. Clin Genet 83, 488-490.

Passemard, S., Kaindl, A.M., and Verloes, A. (2013). Microcephaly. Handb Clin Neurol 111, 129-141.

Patil, D.P., Chen, C.K., Pickering, B.F., Chow, A., Jackson, C., Guttman, M., and Jaffrey, S.R. (2016). m(6)A RNA methylation promotes XIST-mediated transcriptional repression. Nature 537, 369-373.

Pefanis, E., Wang, J., Rothschild, G., Lim, J., Kazadi, D., Sun, J., Federation, A., Chao, J., Elliott, O., Liu, Z.P., et al. (2015). RNA exosome-regulated long non-coding RNA transcription controls super-enhancer activity. Cell 161, 774-789.

Perenthaler, E., Yousefi, S., Niggl, E., and Barakat, T.S. (2019). Beyond the Exome: The Noncoding Genome and Enhancers in Neurodevelopmental Disorders and Malformations of Cortical Development. Front Cell Neurosci 13, 352. 
Pilaz, L.J., Patti, D., Marcy, G., Ollier, E., Pfister, S., Douglas, R.J., Betizeau, M., Gautier, E., Cortay, V., Doerflinger, N., et al. (2009). Forced G1-phase reduction alters mode of division, neuron number, and laminar phenotype in the cerebral cortex. Proc Natl Acad Sci U S A $106,21924-21929$.

Pilz, D.T., Matsumoto, N., Minnerath, S., Mills, P., Gleeson, J.G., Allen, K.M., Walsh, C.A., Barkovich, A.J., Dobyns, W.B., Ledbetter, D.H., et al. (1998). LIS1 and XLIS (DCX) mutations cause most classical lissencephaly, but different patterns of malformation. Hum Mol Genet 7, 2029-2037.

Pinto, L., Mader, M.T., Irmler, M., Gentilini, M., Santoni, F., Drechsel, D., Blum, R., Stahl, R., Bulfone, A., Malatesta, P., et al. (2008). Prospective isolation of functionally distinct radial glial subtypes--lineage and transcriptome analysis. Mol Cell Neurosci 38, 15-42.

Pletikos, M., Sousa, A.M., Sedmak, G., Meyer, K.A., Zhu, Y., Cheng, F., Li, M., Kawasawa, Y.I., and Sestan, N. (2014). Temporal specification and bilaterality of human neocortical topographic gene expression. Neuron 81, 321-332.

Poirier, K., Keays, D.A., Francis, F., Saillour, Y., Bahi, N., Manouvrier, S., Fallet-Bianco, C., Pasquier, L., Toutain, A., Tuy, F.P., et al. (2007). Large spectrum of lissencephaly and pachygyria phenotypes resulting from de novo missense mutations in tubulin alpha $1 \mathrm{~A}$ (TUBA1A). Hum Mutat 28, 1055-1064.

Poirier, K., Saillour, Y., Bahi-Buisson, N., Jaglin, X.H., Fallet-Bianco, C., Nabbout, R., Castelnau-Ptakhine, L., Roubertie, A., Attie-Bitach, T., Desguerre, I., et al. (2010). Mutations in the neuronal ss-tubulin subunit TUBB3 result in malformation of cortical development and neuronal migration defects. Hum Mol Genet 19, 4462-4473.

Pollen, A.A., Nowakowski, T.J., Chen, J., Retallack, H., Sandoval-Espinosa, C., Nicholas, C.R., Shuga, J., Liu, S.J., Oldham, M.C., Diaz, A., et al. (2015). Molecular identity of human outer radial glia during cortical development. Cell 163, 55-67.

Pontious, A., Kowalczyk, T., Englund, C., and Hevner, R.F. (2008). Role of intermediate progenitor cells in cerebral cortex development. Dev Neurosci 30, 24-32.

Pramparo, T., Youn, Y.H., Yingling, J., Hirotsune, S., and Wynshaw-Boris, A. (2010). Novel embryonic neuronal migration and proliferation defects in Dcx mutant mice are exacerbated by Lis1 reduction. J Neurosci 30, 3002-3012.

Preker, P., Nielsen, J., Kammler, S., Lykke-Andersen, S., Christensen, M.S., Mapendano, C.K., Schierup, M.H., and Jensen, T.H. (2008). RNA exosome depletion reveals transcription upstream of active human promoters. Science 322, 1851-1854.

Puno, M.R., and Lima, C.D. (2018). Structural basis for MTR4-ZCCHC8 interactions that stimulate the MTR4 helicase in the nuclear exosome-targeting complex. Proc Natl Acad Sci U S A 115, E5506-E5515.

Rallu, M., Machold, R., Gaiano, N., Corbin, J.G., McMahon, A.P., and Fishell, G. (2002). Dorsoventral patterning is established in the telencephalon of mutants lacking both Gli3 and Hedgehog signaling. Development 129, 4963-4974.

Rauch, S., He, C., and Dickinson, B.C. (2018). Targeted m(6)A Reader Proteins To Study Epitranscriptomic Regulation of Single RNAs. J Am Chem Soc 140, 11974-11981.

Raybaud, C., and Widjaja, E. (2011). Development and dysgenesis of the cerebral cortex: malformations of cortical development. Neuroimaging Clin N Am 21, 483-543, vii.

Reardon, S. (2020). Step aside CRISPR, RNA editing is taking off. Nature 578, 24-27.

Reinsch, S., and Gonczy, P. (1998). Mechanisms of nuclear positioning. J Cell Sci 111 ( Pt 16), 2283-2295.

Resta, N., Susca, F.C., Di Giacomo, M.C., Stella, A., Bukvic, N., Bagnulo, R., Simone, C., and Guanti, G. (2006). A homozygous frameshift mutation in the ESCO2 gene: evidence of intertissue and interindividual variation in Nmd efficiency. J Cell Physiol 209, 67-73.

Rimkus, T.K., Carpenter, R.L., Qasem, S., Chan, M., and Lo, H.W. (2016). Targeting the Sonic Hedgehog Signaling Pathway: Review of Smoothened and GLI Inhibitors. Cancers (Basel) 8 . 
Robinson, D.N., and Spudich, J.A. (2000). Towards a molecular understanding of cytokinesis. Trends Cell Biol 10, 228-237.

Robinson, P.N., Kohler, S., Bauer, S., Seelow, D., Horn, D., and Mundlos, S. (2008). The Human Phenotype Ontology: a tool for annotating and analyzing human hereditary disease. Am J Hum Genet 83, 610-615.

Rolef Ben-Shahar, T., Heeger, S., Lehane, C., East, P., Flynn, H., Skehel, M., and Uhlmann, F. (2008). Eco1-dependent cohesin acetylation during establishment of sister chromatid cohesion. Science 321, 563-566.

Rolfsmeier, M.L., Laughery, M.F., and Haseltine, C.A. (2011). Repair of DNA double-strand breaks induced by ionizing radiation damage correlates with upregulation of homologous recombination genes in Sulfolobus solfataricus. J Mol Biol 414, 485-498.

Ronan, J.L., Wu, W., and Crabtree, G.R. (2013). From neural development to cognition: unexpected roles for chromatin. Nat Rev Genet 14, 347-359.

Rotinen, M., You, S., Yang, J., Coetzee, S.G., Reis-Sobreiro, M., Huang, W.C., Huang, F., Pan, X., Yanez, A., Hazelett, D.J., et al. (2018). ONECUT2 is a targetable master regulator of lethal prostate cancer that suppresses the androgen axis. Nat Med 24, 1887-1898.

Rowitch, D.H., B, S.J., Lee, S.M., Flax, J.D., Snyder, E.Y., and McMahon, A.P. (1999). Sonic hedgehog regulates proliferation and inhibits differentiation of CNS precursor cells. J Neurosci 19, 8954-8965.

Rubin, C.S., and Rosen, O.M. (1975). Protein phosphorylation. Annu Rev Biochem 44, 831887.

Rudnik-Schoneborn, S., Senderek, J., Jen, J.C., Houge, G., Seeman, P., Puchmajerova, A., Graul-Neumann, L., Seidel, U., Korinthenberg, R., Kirschner, J., et al. (2013). Pontocerebellar hypoplasia type 1: clinical spectrum and relevance of EXOSC3 mutations. Neurology 80, 438-446.

Ruiz i Altaba, A., Palma, V., and Dahmane, N. (2002). Hedgehog-Gli signalling and the growth of the brain. Nat Rev Neurosci 3, 24-33.

Sakamoto, M., Hirata, H., Ohtsuka, T., Bessho, Y., and Kageyama, R. (2003). The basic helixloop-helix genes Hesr1/Hey1 and Hesr2/Hey2 regulate maintenance of neural precursor cells in the brain. J Biol Chem 278, 44808-44815.

Sakamoto, M., Iwama, K., Sekiguchi, F., Mashimo, H., Kumada, S., Ishigaki, K., Okamoto, N., Behnam, M., Ghadami, M., Koshimizu, E., et al. (2020). Novel EXOSC9 variants cause pontocerebellar hypoplasia type 1D with spinal motor neuronopathy and cerebellar atrophy. J Hum Genet.

Sakib, M.S., Sokpor, G., Nguyen, H.P., Fischer, A., and Tuoc, T. (2021). Intranuclear immunostaining-based FACS protocol from embryonic cortical tissue. STAR Protoc 2, 100318.

Salomoni, P., and Calegari, F. (2010). Cell cycle control of mammalian neural stem cells: putting a speed limit on G1. Trends Cell Biol 20, 233-243.

Saugier-Veber, P., Marguet, F., Vezain, M., Bucourt, M., Letard, P., Delahaye, A., Pipiras, E., Frebourg, T., Gonzalez, B., and Laquerriere, A. (2020). Pontocerebellar hypoplasia with rhombencephalosynapsis and microlissencephaly expands the spectrum of PCH type 1B. Eur J Med Genet 63, 103814.

Schaeffer, D., Clark, A., Klauer, A.A., Tsanova, B., and van Hoof, A. (2011). Functions of the cytoplasmic exosome. Adv Exp Med Biol 702, 79-90.

Schaeffer, D., Reis, F.P., Johnson, S.J., Arraiano, C.M., and van Hoof, A. (2012). The CR3 motif of Rrp44p is important for interaction with the core exosome and exosome function. Nucleic Acids Res 40, 9298-9307.

Schaeffer, D., Tsanova, B., Barbas, A., Reis, F.P., Dastidar, E.G., Sanchez-Rotunno, M., Arraiano, C.M., and van Hoof, A. (2009). The exosome contains domains with specific endoribonuclease, exoribonuclease and cytoplasmic mRNA decay activities. Nat Struct Mol Biol 16, 56-62. 
Schindelin, J., Arganda-Carreras, I., Frise, E., Kaynig, V., Longair, M., Pietzsch, T., Preibisch, S., Rueden, C., Saalfeld, S., Schmid, B., et al. (2012). Fiji: an open-source platform for biological-image analysis. Nat Methods 9, 676-682.

Schmid, M., and Jensen, T.H. (2019). The Nuclear RNA Exosome and Its Cofactors. Adv Exp Med Biol 1203, 113-132.

Schneeberger, P.E., Nayak, S.S., Fuchs, S., Kutsche, K., and Girisha, K.M. (2020). Roberts syndrome in an Indian patient with humeroradial synostosis, congenital elbow contractures and a novel homozygous splice variant in ESCO2. Am J Med Genet A 182, 2793-2796.

Schneider, C., Kudla, G., Wlotzka, W., Tuck, A., and Tollervey, D. (2012). Transcriptomewide analysis of exosome targets. Mol Cell 48, 422-433.

Schneider, C., Leung, E., Brown, J., and Tollervey, D. (2009). The N-terminal PIN domain of the exosome subunit Rrp44 harbors endonuclease activity and tethers Rrp44 to the yeast core exosome. Nucleic Acids Res 37, 1127-1140.

Schneider, C., and Tollervey, D. (2013). Threading the barrel of the RNA exosome. Trends Biochem Sci 38, 485-493.

Scholey, J.M., Brust-Mascher, I., and Mogilner, A. (2003). Cell division. Nature 422, 746-752.

Schottmann, G., Picker-Minh, S., Schwarz, J.M., Gill, E., Rodenburg, R.J.T., Stenzel, W., Kaindl, A.M., and Schuelke, M. (2017). Recessive mutation in EXOSC3 associates with mitochondrial dysfunction and pontocerebellar hypoplasia. Mitochondrion 37, 46-54.

Schule, B., Oviedo, A., Johnston, K., Pai, S., and Francke, U. (2005). Inactivating mutations in ESCO2 cause SC phocomelia and Roberts syndrome: no phenotype-genotype correlation. Am J Hum Genet 77, 1117-1128.

Schulz, S., Gerloff, C., Ledig, S., Langer, D., Volleth, M., Shirneshan, K., and Wieacker, P. (2008). Prenatal diagnosis of Roberts syndrome and detection of an ESCO2 frameshift mutation in a Pakistani family. Prenat Diagn 28, 42-45.

Schwabova, J., Brozkova, D.S., Petrak, B., Mojzisova, M., Pavlickova, K., Haberlova, J., Mrazkova, L., Hedvicakova, P., Hornofova, L., Kaluzova, M., et al. (2013). Homozygous EXOSC3 mutation c.92G-->C, p.G31A is a founder mutation causing severe pontocerebellar hypoplasia type 1 among the Czech Roma. J Neurogenet 27, 163-169.

Sessa, A., Mao, C.A., Hadjantonakis, A.K., Klein, W.H., and Broccoli, V. (2008). Tbr2 directs conversion of radial glia into basal precursors and guides neuronal amplification by indirect neurogenesis in the developing neocortex. Neuron 60, 56-69.

Sestan, N., and State, M.W. (2018). Lost in Translation: Traversing the Complex Path from Genomics to Therapeutics in Autism Spectrum Disorder. Neuron 100, 406-423.

Shah, J.V., and Cleveland, D.W. (2000). Waiting for anaphase: Mad2 and the spindle assembly checkpoint. Cell 103, 997-1000.

Sharp, D.J., Rogers, G.C., and Scholey, J.M. (2000). Microtubule motors in mitosis. Nature 407, 41-47.

Shikata, Y., Okada, T., Hashimoto, M., Ellis, T., Matsumaru, D., Shiroishi, T., Ogawa, M., Wainwright, B., and Motoyama, J. (2011). Ptch1-mediated dosage-dependent action of Shh signaling regulates neural progenitor development at late gestational stages. Dev Biol 349, 147-159.

Singleton, A.B., Farrer, M., Johnson, J., Singleton, A., Hague, S., Kachergus, J., Hulihan, M., Peuralinna, T., Dutra, A., Nussbaum, R., et al. (2003). alpha-Synuclein locus triplication causes Parkinson's disease. Science 302, 841.

Smith, H.C., Bennett, R.P., Kizilyer, A., McDougall, W.M., and Prohaska, K.M. (2012). Functions and regulation of the APOBEC family of proteins. Semin Cell Dev Biol 23, 258268.

Sokpor, G., Xie, Y., Nguyen, H.P., and Tuoc, T. (2021). Emerging Role of m(6) A Methylome in Brain Development: Implications for Neurological Disorders and Potential Treatment. Front Cell Dev Biol 9, 656849. 
Song, L., Li, Z.Y., Liu, W.P., and Zhao, M.R. (2015). Crosstalk between Wnt/beta-catenin and Hedgehog/Gli signaling pathways in colon cancer and implications for therapy. Cancer Biol Ther 16, 1-7.

Srour, M., Caron, V., Pearson, T., Nielsen, S.B., Levesque, S., Delrue, M.A., Becker, T.A., Hamdan, F.F., Kibar, Z., Sattler, S.G., et al. (2016). Gain-of-Function Mutations in RARB Cause Intellectual Disability with Progressive Motor Impairment. Hum Mutat 37, 786-793.

Srour, M., Chitayat, D., Caron, V., Chassaing, N., Bitoun, P., Patry, L., Cordier, M.P., CapoChichi, J.M., Francannet, C., Calvas, P., et al. (2013). Recessive and dominant mutations in retinoic acid receptor beta in cases with microphthalmia and diaphragmatic hernia. Am $\mathbf{J}$ Hum Genet 93, 765-772.

Staals, R.H., Bronkhorst, A.W., Schilders, G., Slomovic, S., Schuster, G., Heck, A.J., Raijmakers, R., and Pruijn, G.J. (2010). Dis3-like 1: a novel exoribonuclease associated with the human exosome. EMBO J 29, 2358-2367.

Stead, J.A., Costello, J.L., Livingstone, M.J., and Mitchell, P. (2007). The PMC2NT domain of the catalytic exosome subunit Rrp6p provides the interface for binding with its cofactor Rrp47p, a nucleic acid-binding protein. Nucleic Acids Res 35, 5556-5567.

Sterrett, M.C., Enyenihi, L., Leung, S.W., Hess, L., Strassler, S.E., Farchi, D., Lee, R.S., Withers, E.S., Kremsky, I., Baker, R.E., Basrai, M.A., van Hoof, A., Fasken, M.B., Corbett, A.H. (2020). A Budding Yeast Model for Human Disease Mutations in the EXOSC2 Cap Subunit of the RNA Exosome. bioRxiv.

Subramanian, L., Calcagnotto, M.E., and Paredes, M.F. (2019). Cortical Malformations: Lessons in Human Brain Development. Front Cell Neurosci 13, 576.

Sullivan, P.F., and Geschwind, D.H. (2019). Defining the Genetic, Genomic, Cellular, and Diagnostic Architectures of Psychiatric Disorders. Cell 177, 162-183.

Symmons, M.F., Jones, G.H., and Luisi, B.F. (2000). A duplicated fold is the structural basis for polynucleotide phosphorylase catalytic activity, processivity, and regulation. Structure $8,1215-1226$.

Takahashi, S., Araki, Y., Sakuno, T., and Katada, T. (2003). Interaction between Ski7p and Upf1p is required for nonsense-mediated 3'-to-5' mRNA decay in yeast. EMBO J 22, 39513959.

Takahashi, T., Nowakowski, R.S., and Caviness, V.S., Jr. (1995). The cell cycle of the pseudostratified ventricular epithelium of the embryonic murine cerebral wall. J Neurosci 15, 6046-6057.

Tarabykin, V., Stoykova, A., Usman, N., and Gruss, P. (2001). Cortical upper layer neurons derive from the subventricular zone as indicated by Svet1 gene expression. Development $128,1983-1993$.

Tatosyan, K.A., Ustyantsev, I.G., and Kramerov, D.A. (2020). [RNA Degradation in Eukaryotic Cells]. Mol Biol (Mosk) 54, 542-561.

Taverna, E., Gotz, M., and Huttner, W.B. (2014). The cell biology of neurogenesis: toward an understanding of the development and evolution of the neocortex. Annu Rev Cell Dev Biol 30, 465-502.

Taverna, E., and Huttner, W.B. (2010). Neural progenitor nuclei IN motion. Neuron 67, 906914.

Telley, L., Govindan, S., Prados, J., Stevant, I., Nef, S., Dermitzakis, E., Dayer, A., and Jabaudon, D. (2016). Sequential transcriptional waves direct the differentiation of newborn neurons in the mouse neocortex. Science 351, 1443-1446.

Terret, M.E., Sherwood, R., Rahman, S., Qin, J., and Jallepalli, P.V. (2009). Cohesin acetylation speeds the replication fork. Nature 462, 231-234.

Thomaidou, D., Mione, M.C., Cavanagh, J.F., and Parnavelas, J.G. (1997). Apoptosis and its relation to the cell cycle in the developing cerebral cortex. J Neurosci 17, 1075-1085.

Thornton, G.K., and Woods, C.G. (2009). Primary microcephaly: do all roads lead to Rome? Trends Genet 25, 501-510. 
Tomecki, R., Kristiansen, M.S., Lykke-Andersen, S., Chlebowski, A., Larsen, K.M., Szczesny, R.J., Drazkowska, K., Pastula, A., Andersen, J.S., Stepien, P.P., et al. (2010). The human core exosome interacts with differentially localized processive RNases: hDIS3 and hDIS3L. EMBO J 29, 2342-2357.

Toscani, A., Mettus, R.V., Coupland, R., Simpkins, H., Litvin, J., Orth, J., Hatton, K.S., and Reddy, E.P. (1997). Arrest of spermatogenesis and defective breast development in mice lacking A-myb. Nature 386, 713-717.

Toth, A., Ciosk, R., Uhlmann, F., Galova, M., Schleiffer, A., and Nasmyth, K. (1999). Yeast cohesin complex requires a conserved protein, Ecolp $(\mathrm{Ctf} 7)$, to establish cohesion between sister chromatids during DNA replication. Genes Dev 13, 320-333.

Tran, H., Schilling, M., Wirbelauer, C., Hess, D., and Nagamine, Y. (2004). Facilitation of mRNA deadenylation and decay by the exosome-bound, DExH protein RHAU. Mol Cell 13, 101-111.

Trauth, K., Mutschler, B., Jenkins, N.A., Gilbert, D.J., Copeland, N.G., and Klempnauer, K.H. (1994). Mouse A-myb encodes a trans-activator and is expressed in mitotically active cells of the developing central nervous system, adult testis and B lymphocytes. EMBO J 13, 59946005.

Tuoc, T.C., Boretius, S., Sansom, S.N., Pitulescu, M.E., Frahm, J., Livesey, F.J., and Stoykova, A. (2013). Chromatin Regulation by BAF170 Controls Cerebral Cortical Size and Thickness. Developmental Cell 25, 256-269.

Tuoc, T.C., Radyushkin, K., Tonchev, A.B., Pinon, M.C., Ashery-Padan, R., Molnar, Z., Davidoff, M.S., and Stoykova, A. (2009). Selective cortical layering abnormalities and behavioral deficits in cortex-specific Pax6 knock-out mice. J Neurosci 29, 8335-8349.

Tuoc, T.C., and Stoykova, A. (2008). Trim11 modulates the function of neurogenic transcription factor Pax6 through ubiquitin-proteosome system. Genes \& development 22, 1972-1986.

Turrero Garcia, M., Chang, Y., Arai, Y., and Huttner, W.B. (2016). S-phase duration is the main target of cell cycle regulation in neural progenitors of developing ferret neocortex. $\mathbf{J}$ Comp Neurol 524, 456-470.

Ubersax, J.A., and Ferrell, J.E., Jr. (2007). Mechanisms of specificity in protein phosphorylation. Nat Rev Mol Cell Biol 8, 530-541.

Uittenbogaard, M., and Chiaramello, A. (2002). Expression of the bHLH transcription factor Tcf12 (ME1) gene is linked to the expansion of precursor cell populations during neurogenesis. Brain Res Gene Expr Patterns 1, 115-121.

Ulloa, F., and Marti, E. (2010). Wnt won the war: antagonistic role of Wnt over Shh controls dorso-ventral patterning of the vertebrate neural tube. Dev Dyn 239, 69-76.

Unal, E., Heidinger-Pauli, J.M., Kim, W., Guacci, V., Onn, I., Gygi, S.P., and Koshland, D.E. (2008). A molecular determinant for the establishment of sister chromatid cohesion. Science $321,566-569$.

Van Den Berg, D.J., and Francke, U. (1993). Roberts syndrome: a review of 100 cases and a new rating system for severity. Am J Med Genet 47, 1104-1123.

van der Raadt, J., van Gestel, S.H.C., Nadif Kasri, N., and Albers, C.A. (2019). ONECUT transcription factors induce neuronal characteristics and remodel chromatin accessibility. Nucleic Acids Res 47, 5587-5602.

van Dijk, E.L., Schilders, G., and Pruijn, G.J. (2007). Human cell growth requires a functional cytoplasmic exosome, which is involved in various mRNA decay pathways. RNA 13, 10271035.

van Hoof, A., Frischmeyer, P.A., Dietz, H.C., and Parker, R. (2002). Exosome-mediated recognition and degradation of mRNAs lacking a termination codon. Science 295, 22622264. 
van Hoof, A., Lennertz, P., and Parker, R. (2000). Yeast exosome mutants accumulate 3'extended polyadenylated forms of U4 small nuclear RNA and small nucleolar RNAs. Mol Cell Biol 20, 441-452.

Vanacova, S., Wolf, J., Martin, G., Blank, D., Dettwiler, S., Friedlein, A., Langen, H., Keith, G., and Keller, W. (2005). A new yeast poly(A) polymerase complex involved in RNA quality control. PLoS Biol 3, e189.

Vega, H., Trainer, A.H., Gordillo, M., Crosier, M., Kayserili, H., Skovby, F., Uzielli, M.L., Schnur, R.E., Manouvrier, S., Blair, E., et al. (2010). Phenotypic variability in 49 cases of ESCO2 mutations, including novel missense and codon deletion in the acetyltransferase domain, correlates with ESCO2 expression and establishes the clinical criteria for Roberts syndrome. J Med Genet 47, 30-37.

Vega, H., Waisfisz, Q., Gordillo, M., Sakai, N., Yanagihara, I., Yamada, M., van Gosliga, D., Kayserili, H., Xu, C., Ozono, K., et al. (2005). Roberts syndrome is caused by mutations in ESCO2, a human homolog of yeast ECO1 that is essential for the establishment of sister chromatid cohesion. Nat Genet 37, 468-470.

Verloes, A., Le Merrer, M., Davin, J.C., Wittamer, P., Abrassart, C., Bricteux, G., and Briard, M.L. (1992). The orocraniodigital syndrome of Juberg and Hayward. J Med Genet 29, $262-$ 265.

Visel, A., Thaller, C., and Eichele, G. (2004). GenePaint.org: an atlas of gene expression patterns in the mouse embryo. Nucleic Acids Res 32, D552-556.

Vogel, P., Moschref, M., Li, Q., Merkle, T., Selvasaravanan, K.D., Li, J.B., and Stafforst, T. (2018). Efficient and precise editing of endogenous transcripts with SNAP-tagged ADARs. Nat Methods 15, 535-538.

von Dassow, G. (2009). Concurrent cues for cytokinetic furrow induction in animal cells. Trends Cell Biol 19, 165-173.

Wan, J., Yourshaw, M., Mamsa, H., Rudnik-Schoneborn, S., Menezes, M.P., Hong, J.E., Leong, D.W., Senderek, J., Salman, M.S., Chitayat, D., et al. (2012). Mutations in the RNA exosome component gene EXOSC3 cause pontocerebellar hypoplasia and spinal motor neuron degeneration. Nat Genet 44, 704-708.

Wang, F., Wang, L., Zou, X., Duan, S., Li, Z., Deng, Z., Luo, J., Lee, S.Y., and Chen, S. (2019). Advances in CRISPR-Cas systems for RNA targeting, tracking and editing. Biotechnol Adv 37, 708-729.

Wang, J., Duncan, D., Shi, Z., and Zhang, B. (2013). WEB-based GEne SeT AnaLysis Toolkit (WebGestalt): update 2013. Nucleic Acids Res 41, W77-83.

Wang, J., Vasaikar, S., Shi, Z., Greer, M., and Zhang, B. (2017). WebGestalt 2017: a more comprehensive, powerful, flexible and interactive gene set enrichment analysis toolkit. Nucleic Acids Res 45, W130-W137.

Wang, X., Feng, J., Xue, Y., Guan, Z., Zhang, D., Liu, Z., Gong, Z., Wang, Q., Huang, J., Tang, C., et al. (2016). Structural basis of N(6)-adenosine methylation by the METTL3-METTL14 complex. Nature 534, 575-578.

Wang, X., Lu, Z., Gomez, A., Hon, G.C., Yue, Y., Han, D., Fu, Y., Parisien, M., Dai, Q., Jia, G., et al. (2014). N6-methyladenosine-dependent regulation of messenger RNA stability. Nature 505, 117-120.

Wang, X., Zhao, B.S., Roundtree, I.A., Lu, Z., Han, D., Ma, H., Weng, X., Chen, K., Shi, H., and He, C. (2015). N(6)-methyladenosine Modulates Messenger RNA Translation Efficiency. Cell 161, 1388-1399.

Wang, Y., Li, Y., Yue, M., Wang, J., Kumar, S., Wechsler-Reya, R.J., Zhang, Z., Ogawa, Y., Kellis, M., Duester, G., et al. (2018). N(6)-methyladenosine RNA modification regulates embryonic neural stem cell self-renewal through histone modifications. Nat Neurosci 21, 195-206. 
Wasmuth, E.V., and Lima, C.D. (2012). Exo- and endoribonucleolytic activities of yeast cytoplasmic and nuclear RNA exosomes are dependent on the noncatalytic core and central channel. Mol Cell 48, 133-144.

Weissbach, S., Langer, C., Puppe, B., Nedeva, T., Bach, E., Kull, M., Bargou, R., Einsele, H., Rosenwald, A., Knop, S., et al. (2015). The molecular spectrum and clinical impact of DIS3 mutations in multiple myeloma. Br J Haematol 169, 57-70.

Whelan, G., Kreidl, E., Peters, J.M., and Eichele, G. (2012a). The non-redundant function of cohesin acetyltransferase Esco2: some answers and new questions. Nucleus 3, 330-334.

Whelan, G., Kreidl, E., Wutz, G., Egner, A., Peters, J.M., and Eichele, G. (2012b). Cohesin acetyltransferase Esco2 is a cell viability factor and is required for cohesion in pericentric heterochromatin. EMBO J 31, 71-82.

Widagdo, J., and Anggono, V. (2018). The m6A-epitranscriptomic signature in neurobiology: from neurodevelopment to brain plasticity. J Neurochem 147, 137-152.

Wilson, C., Chen, P.J., Miao, Z., and Liu, D.R. (2020). Programmable m(6)A modification of cellular RNAs with a Cas13-directed methyltransferase. Nat Biotechnol 38, 1431-1440.

Wittmann, T., Hyman, A., and Desai, A. (2001). The spindle: a dynamic assembly of microtubules and motors. Nat Cell Biol 3, E28-34.

Wolin, S.L., and Maquat, L.E. (2019). Cellular RNA surveillance in health and disease. Science $366,822-827$.

Wood, J.G., Martin, S., and Price, D.J. (1992). Evidence that the earliest generated cells of the murine cerebral cortex form a transient population in the subplate and marginal zone. Brain Res Dev Brain Res 66, 137-140.

Wu, S.S., Li, Q.C., Yin, C.Q., Xue, W., and Song, C.Q. (2020). Advances in CRISPR/Casbased Gene Therapy in Human Genetic Diseases. Theranostics 10, 4374-4382.

Wyers, F., Rougemaille, M., Badis, G., Rousselle, J.C., Dufour, M.E., Boulay, J., Regnault, B., Devaux, F., Namane, A., Seraphin, B., et al. (2005). Cryptic pol II transcripts are degraded by a nuclear quality control pathway involving a new poly(A) polymerase. Cell 121, 725737.

Xiao, W., Adhikari, S., Dahal, U., Chen, Y.S., Hao, Y.J., Sun, B.F., Sun, H.Y., Li, A., Ping, X.L., Lai, W.Y., et al. (2016). Nuclear m(6)A Reader YTHDC1 Regulates mRNA Splicing. Mol Cell 61, 507-519.

Xie, Y., Castro-Hernandez, R., Sokpor, G., Pham, L., Narayanan, R., Rosenbusch, J., Staiger, J.F., and Tuoc, T. (2019). RBM15 Modulates the Function of Chromatin Remodeling Factor BAF155 Through RNA Methylation in Developing Cortex. Mol Neurobiol 56, 7305-7320.

Yang, L., Chen, J., Li, Y., Wang, Y., Liang, S., Shi, Y., Shi, S., and Xu, Y. (2016). Association between SCAP and SREBF1 gene polymorphisms and metabolic syndrome in schizophrenia patients treated with atypical antipsychotics. World J Biol Psychiatry 17, 467-474.

Yang, Q., Liu, S., Yin, M., Yin, Y., Zhou, G., and Zhou, J. (2015). Ebf2 is required for development of dopamine neurons in the midbrain periaqueductal gray matter of mouse. Dev Neurobiol 75, 1282-1294.

Yang, X., Bayat, V., DiDonato, N., Zhao, Y., Zarnegar, B., Siprashvili, Z., Lopez-Pajares, V., Sun, T., Tao, S., Li, C., et al. (2020). Genetic and genomic studies of pathogenic EXOSC2 mutations in the newly described disease SHRF implicate the autophagy pathway in disease pathogenesis. Hum Mol Genet 29, 541-553.

Yang, Y., Hsu, P.J., Chen, Y.S., and Yang, Y.G. (2018). Dynamic transcriptomic m(6)A decoration: writers, erasers, readers and functions in RNA metabolism. Cell Res 28, 616624.

Yatsuka, H., Hada, K., Shiraishi, H., Umeda, R., Morisaki, I., Urushibata, H., Shimizu, N., Sebastian, W.A., Hikida, T., Ishitani, T., et al. (2020). Exosc2 deficiency leads to developmental disorders by causing a nucleotide pool imbalance in zebrafish. Biochem Biophys Res Commun 533, 1470-1476. 
Yoon, K.J., Ringeling, F.R., Vissers, C., Jacob, F., Pokrass, M., Jimenez-Cyrus, D., Su, Y., Kim, N.S., Zhu, Y., Zheng, L., et al. (2017). Temporal Control of Mammalian Cortical Neurogenesis by m(6)A Methylation. Cell 171, 877-889 e817.

Yoshida, M., Assimacopoulos, S., Jones, K.R., and Grove, E.A. (2006). Massive loss of CajalRetzius cells does not disrupt neocortical layer order. Development 133, 537-545.

Yu, T.W., Mochida, G.H., Tischfield, D.J., Sgaier, S.K., Flores-Sarnat, L., Sergi, C.M., Topcu, M., McDonald, M.T., Barry, B.J., Felie, J.M., et al. (2010). Mutations in WDR62, encoding a centrosome-associated protein, cause microcephaly with simplified gyri and abnormal cortical architecture. Nat Genet 42, 1015-1020.

Zanni, G., Scotton, C., Passarelli, C., Fang, M., Barresi, S., Dallapiccola, B., Wu, B., Gualandi, F., Ferlini, A., Bertini, E., et al. (2013). Exome sequencing in a family with intellectual disability, early onset spasticity, and cerebellar atrophy detects a novel mutation in EXOSC3. Neurogenetics 14, 247-250.

Zhang, B., Kirov, S., and Snoddy, J. (2005). WebGestalt: an integrated system for exploring gene sets in various biological contexts. Nucleic Acids Res 33, W741-748.

Zhang, J., Shi, X., Li, Y., Kim, B.J., Jia, J., Huang, Z., Yang, T., Fu, X., Jung, S.Y., Wang, Y., et al. (2008). Acetylation of Smc3 by Eco1 is required for $\mathrm{S}$ phase sister chromatid cohesion in both human and yeast. Mol Cell 31, 143-151.

Zhang, X.H., Tee, L.Y., Wang, X.G., Huang, Q.S., and Yang, S.H. (2015). Off-target Effects in CRISPR/Cas9-mediated Genome Engineering. Mol Ther Nucleic Acids 4, e264.

Zhao, C., Teng, E.M., Summers, R.G., Jr., Ming, G.L., and Gage, F.H. (2006). Distinct morphological stages of dentate granule neuron maturation in the adult mouse hippocampus. J Neurosci 26, 3-11.

Zhao, J., Li, B., Ma, J., Jin, W., and Ma, X. (2020). Photoactivatable RNA N(6) Methyladenosine Editing with CRISPR-Cas13. Small 16, e1907301.

Zheng, G., Dahl, J.A., Niu, Y., Fedorcsak, P., Huang, C.M., Li, C.J., Vagbo, C.B., Shi, Y., Wang, W.L., Song, S.H., et al. (2013). ALKBH5 is a mammalian RNA demethylase that impacts RNA metabolism and mouse fertility. Mol Cell 49, 18-29.

Zheng, Y., and Pan, D. (2019). The Hippo Signaling Pathway in Development and Disease. Dev Cell 50, 264-282.

Zhong, S., Zhang, S., Fan, X., Wu, Q., Yan, L., Dong, J., Zhang, H., Li, L., Sun, L., Pan, N., et al. (2018). A single-cell RNA-seq survey of the developmental landscape of the human prefrontal cortex. Nature 555, 524-528.

Zou, M., Li, S., Klein, W.H., and Xiang, M. (2012). Brn3a/Pou4f1 regulates dorsal root ganglion sensory neuron specification and axonal projection into the spinal cord. Dev Biol 364, 114-127. 


\section{List of Figures}

Figure 1.1 Mechanisms leading to primary microcephaly in mouse models. ................................... 3

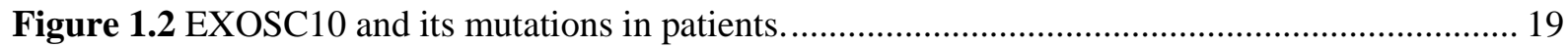

Figure 2.1 Cell Sorting and Gene Expression Profiling of Mouse TBR2+ IPCs ............................... 23

Figure 2.2 Expression of Many Cell-Cycle and Chromatid-Segregation Genes Are Enriched in IPCs25

Figure 2.3 IPC-Enriched Genes Encode for Variety of Signaling Pathway Factors ........................... 27

Figure 2.4 Identification of Novel IPC-Specific Transcription Regulators......................................... 29

Figure 2.5 Mutation of IPC Genes May Underlie Human Cortical Malformation and Intellectual Disability .... 32

Figure 2.6 Lack of ESCO2 Causes Apoptosis of Cortical Progenitors Leading to Disturbance of Cortical

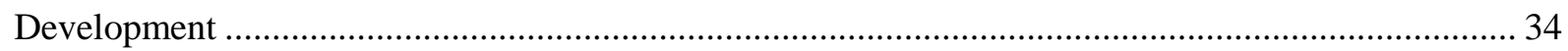

Figure 2.7 Expression of ESCO2 Is Important for Maintenance but Not Generation of IPCs.............. 36

Figure 3.1 The expression of EXOSC10 is indispensable for cortical development. ...........................53

Figure 3.2 Loss of neurons and a reduction in the thickness of cortical layers in postnatal EXOSC10cKO

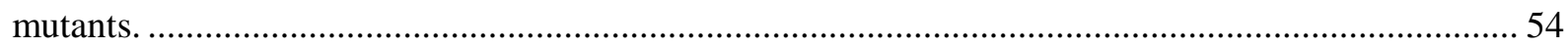

Figure 3.3 A reduced thickness of the cortical plate but normal pools of cortical progenitors in

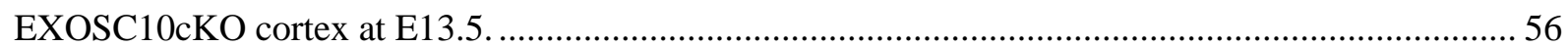

Figure 3.4 EXOSC10 ablation in early cortical progenitors causes massive apoptosis. ....................... 57

Figure 3.5 EXOSC10 controls global gene expression in early cortical development......................... 59

Figure 3.6 EXOSC10 suppresses the expression of P53 pathway AEN and BBC3 genes by degrading

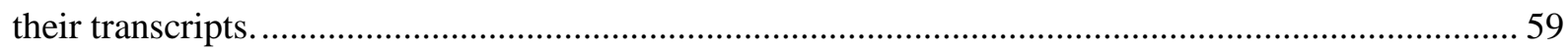

Figure 3.7 EXOSC10 suppresses apoptosis by inhibiting activity of P53 signaling pathway. ........... 61

Figure 3.8 EXOSC10 controls cortical development partly by suppressing P53 signaling activity... 62

Figure 4.1 Loss of EXOSC10 leads to reduction of cortical size and promotes neuronal differentiation

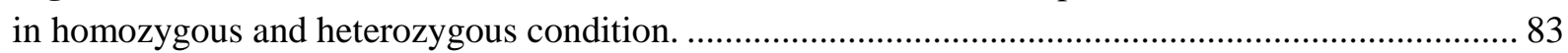

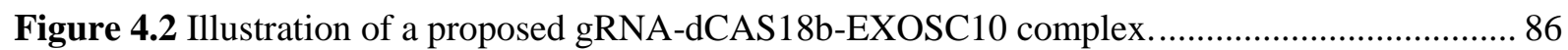

Figure S2.1 (related to Figure 2.1). RNA-seq analysis of mouse TBR2+ IPCs and TBR2-cells......... 40 Figure S2.2 (related to Figure 2.3). TBR2+ IPCs in mouse display prominent apoptotic activity. ..... 41 Figure S2.3 (related to Figure 2.4). Enrichment of transcription factor genes in TBR2+ IPCs. .......... 41 Figure S2.4 (related to Figure 2.5). Many human bIP genes are upregulated in mouse TBR2+ IPCs

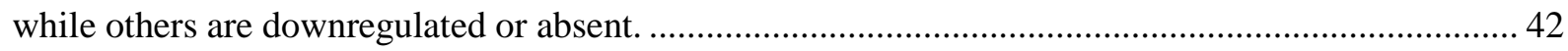
Figure S2.5 (related to Figure 2.6). Expression of ESCO1 and ESCO2 in the developing mouse cortex.

Figure S2.6 (related to Figure 2.7). Deletion of ESCO2 in developing cortex causes depletion of post-

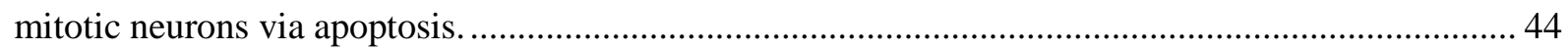

Figure S3.1 Expression of exosome complex subunits in the developing mouse brain...................... 70

Figure S3.2 Expression of EXOSC10 is required for specification of telencephalon......................... 71

Figure S3.3 EXOSC10 expression is not required for cell viability in postnatal cortex. .................... 72

Figure S3.4 Cluster and Heatmap analyses of RNA-seq and RIP-seq samples. ................................ 73

Figure S3.5 Knockout of EXOSC10 using soluble Tat-Cre recombinase in EXOSC10 ${ }^{\text {CoIN/COIN NSCs }}$ and the effects of PFT $\alpha$ treatment on BBC3 expression in the developing cortex.............................. 74 


\section{List of Abbreviations}

A Adenosine

AAV Adeno-associated virus

ac4C N4-acetylcytidine

ADAR adenosine deaminase acting on RNA

AEN Apoptosis enhancing nuclease

AID or AICDA Activation-induced cytidine deaminase

AIR Arginine methyltransferase-interacting RING finger protein

AP Apical progenitor

APOBEC Apolipoprotein B editing complex

aRG Apical radial glial progenitor

ARE AU-rich sequence elements

ARS2 Arsenite-resistance protein 2

ATP Adenosine triphosphate

BAF $\quad$ Brg1/Brm associated factor

BBC3 BCL2 binding component 3

BG Basal ganglia

bHLH Basic-helix-loop-helix

bIP Basal intermediate progenitor

BP Basal progenitor

BRG1 BRM/SWI2 related gene 1

BRM Brahma

C Cytidine 
CASP3

CIdU

$\mathrm{cKO}$

COIN

$\mathrm{CP}$

CR3

CRD-BP

Cre

CRISPR

CTIP2

CUT

CUX1

Cx

DAPI

dCAS13b

DCX

Di

DIS3

DIS3L

DMEM
Caspase 3

Chloro-deoxyuridine

Conditional knockout

Conditional by inversion

Cortical plate

3 Cys residues

Coding region determinant-binding protein

Causes recombination or cAMP-responsive element

Clustered regularly interspaced short palindromic repeats

Chicken ovalbumin upstream promoter transcription factor-interacting protein 2

Cryptic unstable transcript

Cut Like Homeobox 1

Cortex

4,6-dasmindino-2-phenylindol

deactivated CRISPR-associated endoribonuclease $13 \mathrm{~b}$

Dorsal cortex

Diencephalon

Homolog of S. pombe dis3 (chromosome DISjunction)

DIS3-like

Dulbecco's Modified Eagle's Medium 


\begin{tabular}{|c|c|}
\hline DNA & Deoxyribonucleic acid \\
\hline d.p.c. & Days post coitum \\
\hline DSB & Double-strand break \\
\hline dTel & Dorsal telencephalon \\
\hline $\mathrm{E}$ & Embryonic day \\
\hline ECO1 & Establishment of cohesion 1 \\
\hline EDTA & Ethylenediaminetetraacetic acid \\
\hline EGF & Epidermal growth factor \\
\hline eGFP & Enhanced green fluorescent protein \\
\hline eIF3 & Eukaryotic initiation factor 3 \\
\hline EMX1 & Empty spiracles homeobox 1 \\
\hline EN & Early born neurons \\
\hline EOMES & Eomesodermin \\
\hline eRNA & Enhancer ribonucleic acid \\
\hline ESCO1 & Establishment of sister chromatid cohesion $\mathrm{N}$-acetyltransferase 1 \\
\hline $\mathrm{ESCO} 2$ & Establishment of sister chromatid cohesion $\mathrm{N}$-acetyltransferase 2 \\
\hline EXO & Exoribonuclease \\
\hline EXOSC & Exosome component \\
\hline FACS & Fluorescence-activated cell sorting \\
\hline FC & Fold change \\
\hline FGF2 & Fibroblast growth factor \\
\hline $\mathrm{fl}$ & Floxed \\
\hline FOXG1 & Forkhead-Box-Protein G1 \\
\hline
\end{tabular}




\begin{tabular}{|c|c|}
\hline fs & Frameshift \\
\hline GFP & Green fluorescent protein \\
\hline GLI & Glioma-associated oncogene \\
\hline GO & Gene Ontology \\
\hline GSEA & Gene Set Enrichment Analysis \\
\hline HBS1L & Hsp70 subfamily B suppressor 1 like \\
\hline HPO & Human Phenotype Ontology \\
\hline HRDC & RNase D C-terminal \\
\hline HSP & Hereditary spastic paraplegia \\
\hline HUCD & Neuronal $\mathrm{Hu}$ proteins $\mathrm{C}$ and $\mathrm{D}$ \\
\hline I & Inosine \\
\hline $\operatorname{IgG}$ & Immunoglobulin $\mathrm{G}$ \\
\hline IHC & Immunohistochemistry \\
\hline IN & Interneuron \\
\hline IP & Intermediate progenitor \\
\hline IPC & Intermediate progenitor cell \\
\hline ISH & In situ hybridization \\
\hline IUE & In utero electroporation \\
\hline $\mathrm{IZ}$ & Intermediate zone \\
\hline JHS & Juberg-Hayward syndrome \\
\hline $\mathrm{KH}$ & K homolgy \\
\hline Ki67 & Kiel 67 \\
\hline $\mathrm{KO}$ & Knockout \\
\hline
\end{tabular}




\begin{tabular}{|c|c|}
\hline $\mathrm{L}$ & Cortical layer \\
\hline LCX & Lateral cortex \\
\hline LLs & Lower cortical layers \\
\hline $\mathrm{LN}$ & Late born neurons \\
\hline lncRNA & Long non-coding RNA \\
\hline LRP & Low-density lipoprotein receptor-related protein \\
\hline $\mathrm{m} 5 \mathrm{C}$ & 5-methylcytidine \\
\hline m6A & N6-methyladenine \\
\hline m6Am & N6,2'-O-dimethyladenosine \\
\hline $\mathrm{mAB}$ & Monoclonal antibody \\
\hline MCD & Malformation of cortical development \\
\hline MCX & Medial cortex \\
\hline Mes & Mesencephalon \\
\hline METTL & Methyltransferase like \\
\hline MPP6 & M-Phase Phosphoprotein 6 \\
\hline mRNA & Messenger ribonucleic acid \\
\hline MTR & mRNA transport regulator \\
\hline MTREX & MTR4 exosome RNA helicase \\
\hline $\mathrm{MZ}$ & Marginal zone \\
\hline ncRNA & Non-coding ribonucleic acid \\
\hline NEC & Neuroepithelial cell \\
\hline NEUN & Neuronal nuclei antigen \\
\hline NEXT & Nuclear exosome targeting \\
\hline
\end{tabular}




\begin{tabular}{|c|c|}
\hline NGD & No-go decay \\
\hline NMD & Nonsense-mediated decay \\
\hline NS & Not significant \\
\hline NSC & Neural stem cell \\
\hline NSD & Nonstop decay \\
\hline $\mathrm{P}$ & Postnatal day \\
\hline P53 & Tumor protein 53 \\
\hline pAB & Polyclonal antibody \\
\hline PABP2 & Polyadenylate-binding protein 2 \\
\hline PABPN1 & Polyadenylate-binding nuclear protein 1 \\
\hline PAPD5 & Poly(A) RNA polymerase associated domain-containing protein 5 \\
\hline PAX6 & Paired box 6 \\
\hline PAXT & Poly(A) tail exosome targeting \\
\hline PBS & Phosphate Buffer Saline \\
\hline $\mathrm{PCH}$ & Pontocerebellar hypoplasia \\
\hline PCR & Polymerase chain reaction \\
\hline PFT $\alpha$ & Pifithrin- $\alpha$ \\
\hline $\mathrm{PH}$ & Pleckstrin homolgy \\
\hline PIN & PiLT N-terminal \\
\hline PMC2NT & Polycystin $2 \mathrm{~N}$-terminal \\
\hline PROMPT & Promoter upstream transcript \\
\hline PTCH & Patched \\
\hline PUMA & P53-upregulated modulator of apoptosis \\
\hline
\end{tabular}


qPCR

RA

RARB

RARE

$\mathrm{RBM}$

RBS

RG

RIP-seq

RNA

RNA-seq

RNB

rRNA

RRP6

SATB2

SCC

scRNA-seq

SD/THE

SDS

SEM

SHH

SHRF

shRNA

siRNA
Quantitative Polymerase chain reaction

Retinoic acid

Retinoic acid receptor beta

Retinoic acid response element

Ribonucleic acid binding motif

Roberts syndrome

Radial glial cell

Ribonucleic acid immunoprecipitation sequencing

Ribonucleic acid

Ribonucleic acid sequencing

Ribonuclease B

Ribosomal ribonucleic acid

Ribosomal RNA-processing protein

Special AT-Rich Sequence-Binding Protein 2

Sister-chromatid cohesion protein

Single-cell ribonucleic acid sequencing

Syndromic diarrhea/Tricho-hepato-enteric

Sodium dodecyl sulphate

Standard error of the mean

Sonic hedgehog

Short stature, progressive hearing loss, retinitis pigmentosa and distinctive facies

Short hairpin ribonucleic acid

Small interfering ribonucleic acid 


\begin{tabular}{|c|c|}
\hline SKI & Superkiller \\
\hline SKIV2L & Ski2 like RNA helicase \\
\hline SMA & Spinal muscular atrophy \\
\hline SMC & Structural maintenance of chromosomes protein \\
\hline SMO & Smoothened \\
\hline SNCA & $\alpha$-synuclein \\
\hline snoRNA & Small nucleolar ribonucleic acid \\
\hline $\mathrm{SOX} 2$ & SRY-related HMG transcription factor 2 \\
\hline SRRT & Serrate \\
\hline SUFU & Suppressor of Fused \\
\hline SWI/SNF & SWItch/Sucrose Non-Fermentable \\
\hline SVZ & Subventricular zone \\
\hline TBR1 & T-box brain 1 \\
\hline TBR2 & T-box brain 2 \\
\hline Tel & Telencephalon \\
\hline $\mathrm{TF}$ & Transcription factor \\
\hline TRAMP & TRF4/5-AIR1/2-MTR4 Polyadenylation \\
\hline TRF4-2 & Topoisomerase-related function protein 4-2 \\
\hline tRNA & Transmitter ribonucleic acid \\
\hline TTC37 & Tetratricopeptide repeat protein 37 \\
\hline $\mathrm{U}$ & Uridine \\
\hline ULs & Upper cortical layers \\
\hline Veh & Vehicle \\
\hline
\end{tabular}




\begin{tabular}{ll} 
vTel & Ventral telencephalon \\
VZ & Ventricular zone \\
WB & Western blot \\
WDR61 & WD repeat-containing protein 61 \\
WNT & Wingless/Int \\
WT & Wild-type \\
WTAP & Exoribonuclease \\
XRN & YT521-B homology \\
YTH & YTH domain-containing family protein \\
YTHDF & Zinc finger CCHC domain-containing protein \\
ZCCHC & 8-oxo-7,8-dihydroguanosine \\
8-oxoG & Pseudouridine \\
\hline &
\end{tabular}




\section{Acknowledgements}

I wish to thank the members of my thesis advisory committee for their scientific advice helping me to refine and greatly improve my work. Special thanks go to Prof. Dr. Jochen Staiger for giving me the opportunity to work in his institute and his great support during my $\mathrm{PhD}$ time.

I would like to express my deep gratitude to Dr. Tran Tuoc. I feel sincerely grateful for his support and thoughtful supervision. I am looking forward to future projects together!

Further, I would like to thank Dr. Yuanbin Xie, Dr. Godwin Sokpor, Dr. Eman Abbas and Xiaoyi Mao for productive scientific discussions, and their guidance and collaboration during experiments. Many thanks go to our group assistant Linh Pham for her great technical support. But also other current and past members of the Institute of Neuroanatomy are greatly appreciated for various forms of support in the course of my $\mathrm{PhD}$ project. Here, I would also like to thank my friend Anouk Meeuwissen, who did not only help me with injections and proofreading of the thesis but also made the lab and Göttingen a much more fun place.

All other people who collaborated in carrying out various experiments are also appreciated, particularly Sakib Sadman from Prof. Dr. Andre Fischer's group (DZNE).

On top of this, I wish to express my very profound gratitude to my parents Susanne and Martin, my sister Julia, my partner Markus and my daughter Alma for their tender love and unfailing support. In addition, I am grateful to my other family members and friends who have supported me along the way. 


\title{
Curriculum Vitae
}

\author{
Pauline Antonie Ulmke \\ Ludwig-Prandtl-Str. 42, 37077 Göttingen, Germany \\ Phone: +4917671213380 \\ E-Mail: pauline@ulmke.de
}

\section{PERSONAL DETAILS}

Date of Birth: 20 $0^{\text {th }}$ April 1993

Nationality: German

\section{EDUCATION}

Since 2018 Göttingen Graduate Center for Neurosciences, Biophysics and Molecular Biosciences (GGNB), University of Göttingen

Institute of Neuroanatomy, University Medical Center, Kreuzbergring 40, 37075

Göttingen, Germany

Study Course: Molecular Physiology of the Brain (PhD)

Year of graduation: 2021

Title of PhD thesis: Molecular mechanisms underlying cortical (mal)formation: case studies of ESCO2 and EXOSC10

\section{5-2017 University of Göttingen}

Study Course: Developmental, Neural and Behavioural Biology (MSc)

Date of graduation: $29^{\text {th }}$ September 2017, Grade point average: 1.3

Title of Master thesis: Generation of dopaminergic neurons by transdifferentiation

2014 -2015 University of Dundee

Study Course: Molecular Biology (BSc(Hons))

Date of graduation: $25^{\text {th }}$ June 2015, Grade point average: 1.7

Double Degree together with Bonn-Rhein-Sieg University of Applied Sciences Title of Bachelor thesis: Investigation of the role of autophosphorylation on the regulation of the Parkinson's disease associated kinase PINK1

2012-2015 Bonn-Rhein-Sieg University of Applied Sciences

Study Course: Applied Biology (BSc)

Date of graduation: $31^{\text {st }}$ July 2015, Grade point average: 1.9

\section{3-2012 Amos Comenius Gymnasium Bonn}

Abitur: June 2012 (equivalent to A level), Grade point average: 1.8

Main subjects: Biology and Physics 


\section{SKILLS}

\section{Languages}

German (native speaker), English (fluent), Spanish (basics), Latin (basics).

\section{Computer Skills}

Experiences with Adobe Illustrator and Photoshop, Office Software, PowerPoint, ImageJ, GraphPad Prism, Gene Ontology (GO) analysis (with Webgestalt and

\section{Laboratory Skills}

DAVID), Cytoscape, Matlab, LaTeX, R.

Cell culture (primary and continuous), Dissection of insect and mouse brains, Genotyping, Molecular cloning, Transfection, In-utero electroporation, Mitochondrial fractionation, Protein purification, Co-immunoprecipitation, RNAimmunoprecipitation, RNA-sequencing, Immunohistochemistry (IHC), Immunocytochemistry (ICC), PCR, Real-time quantitative PCR (qPCR), Fluorescence-activated cell sorting (FACS), Western blotting, Microscopy.

\section{FURTHER EXPERIENCES}

- Participation in the IGEM (International Genetically Engineered Machine) Team Bonn 2013 (laboratory work and human practice).

- Tutoring and supervision in the practical course Biology for medical students in April/May 2017.

- Participation in the advanced course RNA, Epitranscriptomics and Plasticity from the Neuroscience School of Advanced Studies (NSAS) in Venice in September 2019.

- Involved in organisation of the Encephalon symposium 2019 in Göttingen.

- Work as student representative of my PhD program from July 2019 until now.

\section{SCHOLARSHIPS}

- Deutschlandstipendium 2013/2014

- Deutschlandstipendium 2014/2015

- PROMOS-DAAD 2014

- GGNB travel grant in September 2019

- GGNB bridging fund in July/August 2021

\section{PUBLICATIONS}

\section{First-author papers:}

Ulmke, P. A., Xie, Y., Sokpor, G., Pham, L., Shomroni, O., Berulava, T., ... \& Tuoc, T. (2021). Post-transcriptional regulation by the exosome complex is required for cell survival and forebrain development via repression of P53 signaling. Development, 148(3), dev188276.

Ulmke, P. A., Sakib, M. S., Ditte, P., Sokpor, G., Kerimoglu, C., Pham, L., ... \& Tuoc, T. (2021). Molecular Profiling Reveals Involvement of ESCO2 in Intermediate Progenitor Cell Maintenance in the Developing Mouse Cortex. Stem cell reports, 16(4), 968-984. 


\section{Other papers:}

Narayanan, R., Pham, L., Kerimoglu, C., Watanabe, T., Castro Hernandez, R., Sokpor, G., Ulmke, P.A., Kiszka, K.A., Tonchev, A.B., Rosenbusch, J., et al. (2018). Chromatin Remodeling BAF155 Subunit Regulates the Genesis of Basal Progenitors in Developing Cortex. iScience 4, 109-126.

Pringsheim, M., Mitter, D., Schroder, S., Warthemann, R., Plumacher, K., Kluger, G., Baethmann, M., Bast, T., Braun, S., Buttel, H.M, ... Ulmke, P. A., et al. (2019). Structural brain anomalies in patients with FOXG1 syndrome and in Foxg1+/- mice. Ann Clin Trans1 Neurol 6, 655-668.

Kerimoglu, C., Pham, L., Tonchev, A. B., Sakib, M. S., Xie, Y., Sokpor, G., Ulmke, P. A ... \& Tuoc, T. (2021). H3 acetylation selectively promotes basal progenitor proliferation and neocortex expansion by activating TRNP1 expression. Science Advances. doi: 110.1126/sciadv.abc6792. In Press. 